Schäfers, Markus [Hrsg.]; Welti, Felix [Hrsg.]

\title{
Barrierefreiheit - Zugänglichkeit - Universelles Design. Zur Gestaltung teilhabeförderlicher Umwelten
}

Bad Heilbrunn : Verlag Julius Klinkhardt 2021, 167 S.

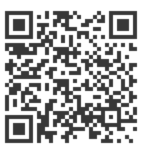

Quellenangabe/ Reference:

Schäfers, Markus [Hrsg.]; Welti, Felix [Hrsg.]: Barrierefreiheit - Zugänglichkeit - Universelles

Design. Zur Gestaltung teilhabeförderlicher Umwelten. Bad Heilbrunn : Verlag Julius Klinkhardt 2021,

167 S. - URN: urn:nbn:de:0111-pedocs-211964 - DOI: 10.25656/01:21196

https://nbn-resolving.org/urn:nbn:de:0111-pedocs-211964

https://doi.org/10.25656/01:21196

in Kooperation mit / in cooperation with:

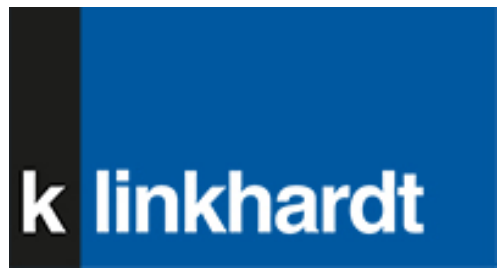

http://www.klinkhardt.de

\section{Nutzungsbedingungen}

Dieses Dokument steht unter folgender Creative Commons-Lizenz: http://creativecommons.org/licenses/by-sa/4.0/deed.de - Sie dürfen das Werk bzw. den Inhalt vervielfältigen, verbreiten und öffentlich zugänglich machen sowie Abwandlungen und Bearbeitungen des Werkes bzw. Inhaltes anfertigen, solange sie den Namen des Autors/Rechteinhabers in der von inm festgelegten Weise nennen und die daraufhin neu entstandenen Werke bzw. Inhalte nur unter Verwendung von Lizenzbedingungen weitergeben, die mit denen dieses Lizenzvertrags identisch, vergleichbar oder kompatibel sind.

Mit der Verwendung dieses Dokuments erkennen Sie die Nutzungsbedingungen an.

\section{Terms of use}

This document is published under following Creative Commons-License: http://creativecommons.org/licenses/by-sa/4.0/deed.en - You may copy, distribute and transmit, adapt or exhibit the work or its contents in public and alter, transform, or change this work as long as you attribute the work in the manner specified by the author or licensor. New resulting works or contents must be distributed pursuant to this license or an identical or comparable license.

By using this particular document, you accept the above-stated conditions of use.

\section{Kontakt / Contact:}

\section{peDOCs}

DIPF | Leibniz-Institut für Bildungsforschung und Bildungsinformation Informationszentrum (IZ) Bildung

E-Mail: pedocs@dipf.de

Internet: www.pedocs.de

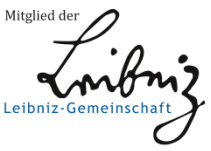




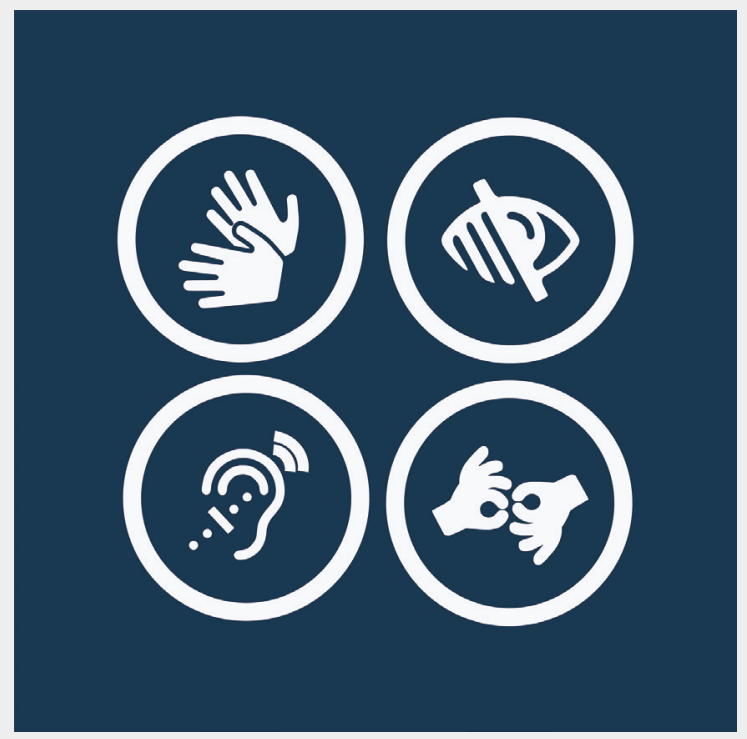

Markus Schäfers

Felix Welti

(Hrsg.)

Barrierefreiheit -

Zugänglichkeit -

Universelles Design

Zur Gestaltung teilhabeförderlicher Umwelten 


\section{Schäfers / Welti \\ Barrierefreiheit - Zugänglichkeit - Universelles Design}



Markus Schäfers

Felix Welti

(Hrsg.)

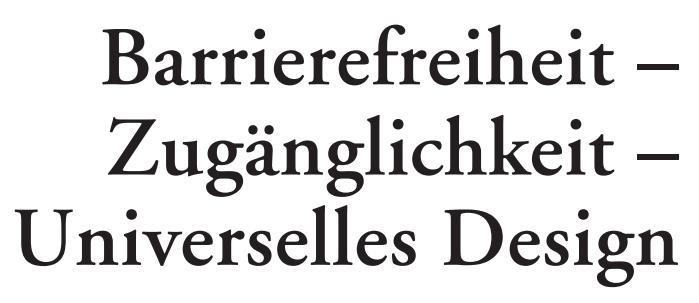

Zur Gestaltung teilhabeförderlicher Umwelten 
Diese Publikation wurde durch den Publikationsfonds der Hochschule FuldaUniversity of Applied Sciences finanziell unterstützt.

Bibliografische Information der Deutschen Nationalbibliothek Die Deutsche Nationalbibliothek verzeichnet diese Publikation in der Deutschen Nationalbibliografie; detaillierte bibliografische Daten sind im Internet abrufbar über http://dnb.d-nb.de.

2021.k. (C) by Julius Klinkhardt.

Satz: Kay Fretwurst, Spreeau.

Grafik Umschlagseite 1: () COOL STUFF / shutterstock.

Druck und Bindung: AZ Druck und Datentechnik, Kempten.

Printed in Germany 2021.

Gedruckt auf chlorfrei gebleichtem alterungsbeständigem Papier.

(c) (i) (0) Die Publikation (mit Ausnahme aller Fotos, Grafiken und Abbildungen) ist veröffent-

licht unter der Creative Commons-Lizenz: CC BY-SA 4.0 International https://creativecommons.org/licenses/by-sa/4.0/

ISBN 978-3-7815-5856-4 digital

doi.org/10.35468/5856

ISBN 978-3-7815-5857-1 ePub

ISBN 978-3-7815-2418-7 print 


\section{Inhalt}

Markus Schäfers und Felix Welti

Vorwort

\section{Theoretische Grundlegung}

Felix Welti

Zum Verständnis von Barrieren und Barrierefreiheit aus rechtswissenschaftlicher Sicht

Marianne Hirschberg

Barrieren als gesellschaftliche Hindernisse -

Sozialwissenschaftliche Überlegungen

Oliver Sträter

Universal Design - Gestaltung der Zugänglichkeit von Arbeitssystemen

für Menschen mit Behinderung 36

\section{Empirische Erfassung}

Friedrich Dieckmann

Verständnis und empirische Erfassung von Barrieren aus

ökologisch-psychologischer Sicht

Markus Schäfers und Viviane Schachler

Barrieren erfragen - Herausforderungen der empirischen Erfassung von Barrieren im Rahmen standardisierter Interviews

\section{Anwendungsfelder}

\section{Matthias Schmidt-Ohlemann}

Barrierefreie Gesundheitsversorgung - zwischen Zugänglichkeit

allgemeiner Versorgung und Notwendigkeit besonderer Einrichtungen

Leonora Micah Jordan

Barrierefreie Beratung - Räume der Begegnung niedrigschwellig gestalten 
Tanja Freifrau Schenck zu Schweinsberg (geb. Lück) und Dominik Rupprecht Barrierefreie dialogorientierte Teilhabeplanung am Beispiel des Integrierten Teilhabeplans (ITP)

Barbara Klein

Assistive und andere Technologien

\section{Planungs- und Gestaltungsprozesse}

Christophe Kunze

Nutzerorientierte und partizipative Ansätze in Gestaltungs- und Aneignungsprozessen von teilhabefördernder Technik

Markus Rebstock

Barrierefreiheit in Planungsprozessen 143

Autorinnen und Autoren 157

Beschreibung für Abbildungen und Tabellen 160 


\section{Vorwort}

Barrierefreie Umwelten ermöglichen Menschen mit Beeinträchtigungen, am gesellschaftlichen Leben teilhaben zu können. Aber was genau bedeutet Barrierefreiheit? Anders gewendet: Was macht eine Gegebenheit in der Umwelt zu einer Barriere? Welche Aspekte entscheiden darüber, ob Umwelten förderlich oder hinderlich für die gesellschaftliche Teilhabe sind? Wie lassen sich diese Aspekte messen, beurteilen und klassifizieren?

Der Begriff der Barriere ist untrennbar mit einem modernen Behinderungsverständnis verbunden. Die Behindertenrechtskonvention der Vereinten Nationen (UNBRK) und die Internationale Klassifikation der Funktionsfähigkeit, Behinderung und Gesundheit (ICF) vermitteln ein neues Verständnis von Behinderung, das den sozialen Einflussfaktoren und den gesellschaftlichen Bedingungen bei der Entstehung von Behinderung vermehrt Rechnung trägt. Bereits Art. 1 UN-BRK enthält Bezüge zum Begriff der Barriere:

„Zu den Menschen mit Behinderungen zählen Menschen, die langfristige körperliche, seelische, geistige oder Sinnesschädigungen haben, welche sie in der Wechselwirkung mit verschiedenen Barrieren an der vollen, wirksamen und gleichberechtigten Teilhabe an der Gesellschaft hindern können."

Die Behinderungsdefinition des Behindertengleichstellungsgesetzes (BGG) und des Sozialgesetzbuchs (SGB) IX nimmt diesen Gedanken auf und spricht von Beeinträchtigungen, die "in Wechselwirkung mit einstellungs- und umweltbedingten Barrieren “( $\$ 3$ BGG; $\$ 2$ Abs. 1 SGB IX) einen Menschen mit Behinderungen an der Teilhabe hindern.

Was unter einer Barriere zu verstehen ist, ist allerdings mit Inhalt zu füllen. So kann Barrierefreiheit unterschiedlich weit oder eng bzw. abstrakt oder konkret gedacht werden. Konzepte des Universal Design oder des Design for All beziehen sich auf bauliche Einrichtungen, Gebrauchsgegenstände, Informationstechnologien usw. und versprechen deren Zugänglichkeit und Nutzbarkeit für alle Personen unabhängig von der Art der Beeinträchtigung oder anderen Merkmalen. Wer aber sind „all“"? Ist dieser Anspruch erfüllbar?

Zuweilen werden auch negative Einstellungen (wie Ressentiments gegenüber Menschen mit Behinderungen) unter den Begriff der Barriere gefasst. Dies wirft weitere Fragen auf: Ist es geboten, Barrieren so weit zu verstehen, oder wird der Begriff damit überstrapaziert? Wen adressiert ein solcher Barrierebegriff? Die ohnehin bestehende Frage der Zuordnung von Verantwortlichkeiten für den Abbau 
von Barrieren wird dadurch noch verschärft: Wer ist dazu berufen, umwelt- und einstellungsbedingte Barrieren zu beseitigen? Wie weit gehen rechtliche Verpflichtungen? Welche Arten von Interventionen sind überhaupt denkbar, um gestaltete Umwelten und gesellschaftliche Einstellungen zu verändern?

Mit diesen und weiteren Fragen beschäftigt sich der vorliegende Band in interdisziplinärer Perspektive. Die Idee für das Buch geht zurück auf einen Expert*innenWorkshop des Forschungsverbunds für Sozialrecht und Sozialpolitik (FoSS) der Hochschule Fulda und der Universität Kassel mit Unterstützung der Hochschule der Gesetzlichen Unfallversicherung in Bad Hersfeld im März 2019.

Die versammelten Beiträge zeigen die Vielschichtigkeit und Komplexität des Themas Barrierefreiheit, zugleich den Gewinn einer interdisziplinären Betrachtung. Der Band soll der Diskussion um Barrieren und Barrierefreiheit - einem noch wenig erforschten Gebiet - Substanz verleihen und zum interdisziplinären Dialog inspirieren.

Fulda und Kassel,

Markus Schäfers und Felix Welti 


\title{
Theoretische Grundlegung
}

\author{
Felix Welti
}

\section{Zum Verständnis von Barrieren und Barrierefreiheit aus rechtswissenschaftlicher Sicht}

\section{Vorbemerkung}

Eine rechtswissenschaftliche Sicht auf die Begriffe Barriere und Barrierefreiheit richtet sich - grundlagenorientiert und hoffentlich interdisziplinär und zur sozialen Praxis anschlussfähig (vgl. Welti 2015a, 17) - darauf, wie die Rechtsbegriffe konstituiert werden und wie sich diese Begriffsverwendung zur Analyse anderer Disziplinen verhält. In der anwendungsorientierten immanent rechtswissenschaftlichen Betrachtung befasst sie sich damit, welchen Gebrauch Gerichte und Behörden von dem Rechtsbegriff in seiner Anwendung machen und richtigerweise machen sollten. Dabei kann auch betrachtet werden, wie Akteure des Rechtssystems - unter anderem Menschen mit Behinderungen und ihre Verbände - die auf Barrierefreiheit bezogenen Rechtsnormen nutzen und nutzen könnten. Insoweit verknüpft eine rechtswissenschaftliche Betrachtung empirische und normative Elemente im Erkenntnisprozess. Das allerdings teilt sie letztlich mit allen Wissenschaften, die Barrieren und Barrierefreiheit thematisieren, da schon die Kategorien - zumindest auch - in normativer Absicht gebildet worden sind. Zum Teil wird in wissenschaftlichen Texten - etwa der Soziologie, Pädagogik oder Ingenieurwissenschaften - die normative Entscheidung dem Recht zugeordnet, indem Rechtsnormen (wie Art. 9 UN-BRK) zur Begründung des Erkenntnisinteresses angeführt werden. Dies ist umso einfacher und häufiger geworden, als Barrierefreiheit Gegenstand einer Menschenrechtskonvention und vielfältiger Normen des einfachen Rechts geworden ist. Frühere Thematisierungen in Politik und Wissenschaft haben sich eher aus dem Ziel der Rehabilitation und damit stärker aus einem sozialmedizinisch und sozialpolitisch geprägten Kontext abgeleitet (Bösl 2012; Bösl 2016, 100-105). Die intensivere Befassung mit Barrieren und Barrierefreiheit steht aber erkennbar im Übergang von einem eher individuell-medizini- 
schen zu einem sozialen und rechtebasierten Verständnis von Behinderung (Heyer 2015, 23). Ein solches kann die Methoden und Erkenntnisse der Rechtswissenschaft nutzen, ohne ihnen die Deutungshoheit überlassen zu müssen.

\section{Barrieren und Behinderung}

In Art. 1 Satz 2 UN-BRK ist festgehalten, dass zu den Menschen mit Behinderungen Menschen zählen, die langfristige körperliche, seelische, geistige oder Sinnesbeeinträchtigungen haben, welche sie in Wechselwirkung mit verschiedenen Barrieren an der vollen, wirksamen und gleichberechtigten Teilhabe an der Gesellschaft hindern können. Dieser Satz - oft als „Behinderungsbegriff“ verstanden - ist in der Konvention ausdrücklich nicht in Artikel 2 „Begriffsbestimmungen“, sondern in Artikel 1 „Zweck" verortet. Ausweislich der Präambel (lit. e) ist der Inhalt von Art. 1 Satz 2 als „Erkenntnis“ ausgewiesen, die im Zusammenhang damit steht, dass sich das Verständnis von Behinderung ständig weiterentwickelt. Damit wird eine doppelte Wechselwirkung, nämlich zwischen Individuum und Gesellschaft und zwischen Norm und Wirklichkeit, zugleich als erkenntnistheoretische Grundlage wie als Zweck des Menschenrechtsschutzes für Menschen mit Behinderungen ausgewiesen. Art. 1 Satz 2 UN-BRK hat Elemente aus dem Diskurs aufgenommen, der zur International Classification of Functioning, Disability and Health (ICF) geführt hatte, und in dem Barrieren als Umweltfaktoren in die Klassifikation individueller Behinderungen Eingang gefunden haben (vgl. Hirschberg 2009, 177-190). Die offene Beschreibung in Art. 1 Satz 2 UN-BRK war ein Kompromiss angesichts der Abwägung zwischen Risiken und Chancen einer festen Definition, der sichtbar macht, dass der Diskurs auf Dauer gestellt sein muss. Auch dies steht für den innovativen Charakter der UN-BRK, die Impulse für eine soziale und inklusive Menschenrechtstheorie geben kann (Degener 2019, 501-506).

Auf der Ebene des einfachen Rechts werden allerdings zur Anwendbarkeit feste Definitionen benötigt. Die 2001 und 2002 in $\$ 2$ Abs. 1 SGB IX und $₫ 3$ BGG kodifizierten Definitionen von Behinderung nahmen ausweislich der Begründung den Impuls der ICF auf (BT-Drucks. 14/5074, 98). Mit dem Gesetz zur Weiterentwicklung des Behindertengleichstellungsgesetzes hat der deutsche Gesetzgeber 2016 das Ziel verfolgt, den Wortlaut des Behinderungsbegriffs an die UN-BRK anzupassen, um das Bewusstsein für das zeitgemäße Verständnis von Behinderung zu schärfen und die Rechtsanwendung in der Praxis zu unterstützen (BT-Drucks. 18/7824, 21, 33f.) und folgt damit einem Vorschlag der Evaluation des BGG (Welti u.a. 2014, 434-439). $\$ 3$ BGG lautet nun:

„Menschen mit Behinderungen im Sinne dieses Gesetzes sind Menschen, die langfristige körperliche, seelische, geistige oder Sinnesbeeinträchtigungen haben, welche sie in Wechselwirkung mit einstellungs- und umweltbedingten Barrieren an der gleichberech- 
tigten Teilhabe an der Gesellschaft hindern können. Als langfristig gilt ein Zeitraum, der mit hoher Wahrscheinlichkeit länger als sechs Monate andauert.“

Sehr ähnlich und mit ähnlicher Begründung wurde mit dem Bundesteilhabegesetz zum 1.1.2018 \$2 Abs. 1 SGB IX neu gefasst (BT-Drucks. 18/9522, 227). Während Art. 1 Satz 2 UN-BRK von verschiedenen Barrieren spricht, ist die Unterscheidung in einstellungs- und umweltbedingte Barrieren aus der Präambel (lit. e) der UN-BRK übernommen. Sie entspricht nicht der in der ICF genutzten Aufteilung zwischen Umweltfaktoren und personbezogenen Faktoren, welche jeweils Barrieren oder fördernde Faktoren sein können. Umweltfaktoren als Barrieren können danach zum Beispiel eine unzugängliche physische Umgebung, das Fehlen unterstützender Technik oder negative Einstellungen von Menschen sein (Hirschberg 2009, 191). Welche Barrieren jeweils wie in eine rechtliche Betrachtung einzubeziehen sind, könnte nach Regelungsbereich zu differenzieren sein. Während das Behindertengleichstellungsrecht auf die Umweltfaktoren von Menschen mit Behinderungen zielt, ist das Rehabilitations- und Teilhaberecht zumindest auch auf die Gestaltung personbezogener Faktoren gerichtet. Das BGG befasst sich mit den Einstellungen anderer Menschen, das SGB IX auch mit den Einstellungen der Menschen mit Behinderungen selbst, ohne darauf beschränkt zu sein, so z.B. $\$ 49$ Abs. 6 Satz 2 SGB IX, wonach Leistungen zur Teilhabe am Arbeitsleben sowohl die eigene Behinderungsverarbeitung und Motivation als auch die Information und Beratung von Angehörigen, Partner*innen, Vorgesetzten und Kolleg*innen umfassen können. In $₫ 2$ Abs. 1 SGB IX deutet der Kontext individueller Leistungsansprüche und Statusrechte darauf hin, dass die Barrieren auf je individuelle Menschen mit Behinderungen zu beziehen sind, während im Kontext von $₫ 3$ BGG sowohl individuelle Ansprüche auf Diskriminierungsschutz als auch strukturelle Vorkehrungen zu betrachten sind, die auf Menschen mit Behinderungen als Kollektiv zielen. Vor allem letztere werden mit dem Begriff der Barrierefreiheit belegt.

\section{Zugänglichkeit und Barrierefreiheit}

\subsection{Barrierefreiheit im deutschen Recht}

Barrierefreiheit ist seit 2016 in $₫ 4$ BGG definiert:

„Barrierefrei sind bauliche und sonstige Anlagen, Verkehrsmittel, technische Gebrauchsgegenstände, Systeme der Informationsverarbeitung, akustische und visuelle Informationsquellen und Kommunikationseinrichtungen sowie andere gestaltete Lebensbereiche, wenn sie für Menschen mit Behinderungen in der allgemein üblichen Weise, ohne besondere Erschwernis und grundsätzlich ohne fremde Hilfe auffindbar, zugänglich und nutzbar sind. Hierbei ist die Nutzung behinderungsbedingt notwendiger Hilfsmittel zulässig."

Die Formulierung wurde 2016 nur um das Wort „auffindbar“ sowie um den zweiten Satz ergänzt. Sie geht im Übrigen auf die Einführung des BGG im Jahr 2002 
zurück, das maßgeblich von Gruppen aus der Behindertenbewegung angeregt worden ist (vgl. Frehe 2013; Theben 2018). Dort wurde in der Gesetzesbegründung ausgeführt:

„Die Definition löst die Begriffe ,behindertengerecht' und ,behindertenfreundlich“ ab, die in der Kombination von ,behindert' und ,gerecht' oder ,freundlich' falsche Assoziationen der besonderen Zuwendung zu behinderten Menschen auslösen können. Vielmehr geht es im Sinne eines, universal design' um eine allgemeine Gestaltung des Lebensumfeldes für alle Menschen, die möglichst niemanden ausschließt und von allen gleichermaßen genutzt werden kann. Dieser Gedanke, einer wenn immer möglichen Vermeidung von Sonderlösungen zugunsten einer die Bedarfe behinderter Menschen selbstverständlich einbeziehenden gesellschaftlichen Gestaltung, entspricht einer modernen Auffassung von Architektur und Design." (BT-Drs. 14/7420, 24)

Im Weiteren heißt es in der Begründung, dass die Gestaltung nicht auf die spezielle Ausprägung einer Behinderung, sondern auf eine möglichst allgemeine Nutzbarkeit abgestimmt werden solle. Barrierefreiheit sei eine Zielvorgabe für gestaltete Lebensbereiche, die häufig nur in begrenztem Umfange erreicht werden könne und deren Standards einem ständigen Wandel unterworfen seien (BT-Drs. 14/7420, 25). Es wird deutlich, dass Barrierefreiheit in diesem Sinne auf die Gesamtheit der Menschen mit Behinderungen zielt. Sie typisiert die zu vermeidenden Barrieren für eine unbestimmte Vielzahl von Menschen, die durch sie behindert werden können. Zumindest ohne weitere Konkretisierungen ist sie in den Normtyp des zu optimierenden Prinzips einzuordnen. Zur strikten Regel kann sie erst durch weitere Konkretisierungen werden (zur Unterscheidung: Alexy 1994, 71).

Die Ergänzungen des Jahres 2016 werden in den Gesetzgebungsmaterialien als Klarstellungen und Anpassungen bezeichnet (BT-Drs. 18/7824, 34), wobei der zweite Satz sprachlich nicht ganz geglückt erscheint. Gemeint ist wohl, dass behinderungsbedingt notwendige Hilfsmittel zulässig sein müssen, wenn sie notwendig sind, um gestaltete Lebensbereiche nutzen zu können. Damit wird die Schnittstelle zwischen individuellen Barrieren und Hilfsmittel als personbezogenen Förderfaktoren zu generellen Umweltgestaltungen angesprochen.

Die Definition von Barrierefreiheit ist für eine auf Gleichstellung von Menschen mit Behinderungen zielende Kodifikation nicht zwingend. So enthalten das Behindertengleichstellungsgesetz der Schweiz vom 13.12.2002 und das BundesBehindertengleichstellungsgesetz von Österreich vom 10.8.2005 keine solche Definition. Die Behindertengleichstellungsgesetze aller sechzehn deutschen Länder folgen indes heute der Regelungstechnik des Bundesgesetzes und beinhalten fast gleichlautende Definitionen von Barrierefreiheit. Die ersten Kodifikationen in Berlin und Sachsen-Anhalt vor dem BGG des Bundes hatten sie noch nicht enthalten.

Die Barrierefreiheit im Sinne von $\$ 4$ BGG bezieht sich ersichtlich nicht auf die Gesamtheit der einstellungs- und umweltbedingten Barrieren im Sinne von $₫ 3$ BGG. 
Es erscheint plausibel, dass die einstellungsbedingten Barrieren, verstanden als solche, die sich aus Einstellungen und Verhalten von Menschen ergeben, Gegenstand von Benachteiligungsverboten in $\$ 7$ BGG und $\$ 1$ AGG sind, während die Barrierefreiheit nach $\$ 4$ BGG sich auf gestaltbare umweltbedingte Barrieren bezieht.

\subsection{Zugänglichkeit im internationalen Recht}

In der 2006 von der UN-Generalversammlung beschlossenen und Ende 2008 von der Bundesrepublik Deutschland ratifizierten UN-BRK findet sich in Art. 2 UN-BRK eine Definition universellen Designs als Design von Produkten, Umfeldern, Programmen und Dienstleistungen in der Weise, dass sie von allen Menschen möglichst weitgehend ohne eine Anpassung oder ein spezielles Design genutzt werden können, was Hilfsmittel nicht ausschließt. Die Vertragsstaaten sind nach Art. 4 Abs. 1 lit. f I UN-BRK verpflichtet, Forschung und Entwicklung für universelles Design (Schaumberg 2018) zu fördern.

In Art. 3 lit $\mathrm{f}$ wird die Zugänglichkeit (englisch: accessibility, französisch: accessibilité) als allgemeiner Grundsatz der Konvention ausgewiesen. Dieser ist näher ausgeformt in Art. 9 UN-BRK. Dort heißt es, dass die Vertragsstaaten um Menschen mit Behinderungen eine unabhängige Lebensführung und die volle Teilhabe in allen Lebensbereichen zu ermöglichen, geeignete Maßnahmen treffen mit dem Ziel, für Menschen mit Behinderungen gleichberechtigten Zugang zur physischen Umwelt, zu Transportmitteln, Information und Kommunikation sowie zu anderen Einrichtungen und Diensten, die der Öffentlichkeit offenstehen und für sie bereitgestellt werden, zu gewährleisten. Diese Maßnahmen schließen die Feststellung und Beseitigung von Zugangshindernissen und -barrieren ein und gelten unter anderem für Gebäude, Straßen, Transportmittel sowie andere Einrichtungen einschließlich Schulen, Wohnhäuser, medizinische Einrichtungen und Arbeitsstätten, Informations-, Kommunikations- und andere Dienste (vgl. Welti 2012).

Art. 9 UN-BRK wird vom Ausschuss für die Rechte von Menschen mit Behinderungen im General Comment No 2 vom 22.5.2014 erläutert (CRPD/C/GC/2). Darin wird verdeutlicht, dass Zugänglichkeit als Voraussetzung von Teilhabe und des Genusses von Rechten gesehen wird (Ziffer 1). Entsprechend der historischen Bezugnahmen in der Entwicklung des Antidiskriminierungsrechts (vgl. Heyer 2015, 51) wird auf das Recht auf Zugang zu jedem Ort oder Dienst, der für die Benutzung durch die Öffentlichkeit vorgesehen ist, in Art. 5 lit. f des Internationalen Übereinkommens zur Beseitigung jeder Form von Rassendiskriminierung vom 7.3.1966 hingewiesen (Ziffer 3). Diese Vorschrift, so auch GC 2, zielt auf einstellungsbedingte Barrieren. Es wird jedoch darauf hingewiesen, dass technische und umweltbedingte Barrieren in den meisten Fällen menschgemacht sind und mit der sozialen und kulturellen Entwicklung und mit dem Informationsstand zusammenhängen. Daher wird der Zusammenhang zum Diskriminierungs- 
verbot in Art. 5 UN-BRK (Ziffer 34) und zum Gebot der Bewusstseinsbildung in Art. 8 UN-BRK (Ziffer 35) herausgestellt.

Das Prinzip der Zugänglichkeit wurzelt in der modernen Menschenrechtsdiskussion, gleichermaßen Freiheitsrechte und Gleichheitsrechte, bürgerliche und politische, wirtschaftliche, soziale und kulturelle Rechte betreffend. Es verdeutlicht die Staatenpflicht zur Gewährleistung der Menschenrechte („duty to fulfil“) (vgl. zu den Staatenpflichten: Banafsche 2015, 61). Ausgeformt wurde dies etwa im General Comment No 14 des Sozialpakt-Ausschusses zum Recht auf Gesundheit nach Art. 12 Sozialpakt vom 11.8.2000 (E./C.12/2000/4). Dort wird Zugänglichkeit von Gesundheitsleistungen (vgl. Hlava 2018, 67) in vier Dimensionen systematisiert: Nicht-Diskriminierung, physische Zugänglichkeit, ökonomische Zugänglichkeit (Erschwinglichkeit) und Informationszugänglichkeit.

\subsection{EU-Recht}

Die Europäische Union hat 2010 die UN-BRK ratifiziert, so dass diese zur Auslegung des gesamten EU-Rechts herangezogen werden kann. Namentlich durch die Gleichbehandlungsrahmenrichtlinie RL 2000/78/EG, in der in Art. 5 RL 2000/78/EG erstmals das Gebot angemessener Vorkehrungen für Menschen mit Behinderungen an die Arbeitgeber normiert worden ist, hat das EU-Recht erheblichen Einfluss auf das nationale Arbeitsrecht, in Deutschland vor allem auf das AGG und das Sozialrecht der Arbeitsförderung (Welti u.a. 2019; Eichenhofer 2019).

Auch in einigen Gebieten des den potenziell grenzüberschreitenden Verkehr betreffenden Öffentlichen Rechts und Privatrechts sind Anforderungen zur Barrierefreiheit durch das EU-Recht getroffen und vereinheitlicht worden, so durch VO EG 1107/2006 über die Rechte von behinderten Flugreisenden und von Flugreisenden mit eingeschränkter Mobilität (Kuhn-Zuber 2018), die VO EU 1177/2010 über die Fahrgastrechte im See- und Binnenschiffsverkehr und die VO 181/2011 über die Fahrgastrechte im Kraftomnibusverkehr und die VO 1371/2007 über die Fahrgastrechte im Eisenbahnverkehr (Fuerst 2018, 839). Barrierefreiheit und Universelles Design werden auch im EU-Vergaberecht als Kriterien genutzt (Schaumberg 2018, 1130).

Ein neuer Schub EU-rechtlichen Einflusses erfolgt durch die Richtlinie 2019/882/ EU über die Barrierefreiheitsanforderungen für Produkte und Dienstleistungen, deren Anforderungen bis zum 28. Juni 2022 ins deutsche Recht umgesetzt sein müssen. Die Begründung nimmt ausdrücklich auf die UN-BRK Bezug. Die Richtlinie gilt für das ganze Wirtschaftsleben. Sie enthält keine eigene Definition von Zugänglichkeit und Barrierefreiheit, sondern gibt vor, dass Waren und Dienstleistungen so auszugestalten sind, dass Menschen mit Behinderungen sie voraussichtlich maximal nutzen. Hierzu werden in Anhängen spezifische technische Anforderungen und Informationspflichten und ein Konformitätsbewer- 
tungsverfahren (CE-Kennzeichnung) festgelegt. Die RL 2019/882/EU passt sich insoweit in das EU-Verbraucherschutzrecht ein.

\section{Barrierefreiheit und Diskriminierungsverbot}

Das Verhältnis zwischen dem Gebot der Barrierefreiheit und dem Verbot der Diskriminierung wegen Behinderung ist zu bestimmen. Hier kommt es gerade deshalb zu Überschneidungen und Differenzierungsbedarf, weil das Diskriminierungsverbot bei Menschen mit Behinderungen als Handlungspflicht zu angemessenen Vorkehrungen (Art. 5 Abs. 3 UN-BRK) ausgeprägt sein kann. Hierauf geht der Ausschuss in seinem General Comment (GC) No 6 vom 26.4.2018 zu Art. 5 UN-BRK (CRPD/C/GC/6) ein. Er erläutert (Ziffer 41), dass Zugänglichkeit und angemessene Vorkehrungen zwei unterschiedliche Konzepte von Gleichheitsrechten und Politiken seien: Zugänglichkeitspflichten sind mit Gruppen verknüpft und müssen nach und nach, aber unbedingt implementiert werden; angemessene Vorkehrungen sind individualisierte Pflichten, die unmittelbar anzuwenden sind und durch Unverhältnismäßigkeit begrenzt sein können. Weil die allmähliche Verwirklichung von Zugänglichkeit Zeit brauchen kann, kann zwischenzeitlich die Pflicht zu angemessenen Vorkehrungen genutzt werden, um Individuen $\mathrm{Zu}-$ gang zu gewährleisten (Ziffer 42).

Seit der Reform von 2016 wird für das Benachteiligungsverbot gegenüber Bundesbehörden und Landesbehörden, die Bundesrecht ausführen in $\$ 7$ Abs. 1 Satz 4 BGG widerleglich vermutet, dass eine Benachteiligung vorliegt, wenn gegen eine Verpflichtung zur Barrierefreiheit verstoßen wird. Damit wird klargestellt, dass immer dort, wo Barrierefreiheit der Verwaltung geboten wäre, aber (noch) nicht besteht, ein Anspruch auf angemessene Vorkehrungen besteht, denn die Vermutung der Benachteiligung kann gerade durch angemessene Vorkehrungen widerlegt werden (BT-Drs. 18/7824, 34). Relevant kann dies insbesondere bei Sozialleistungsträgern der Bundes-, Landes- und Kommunalverwaltung werden: Sie führen Bundesrecht aus, sind also im Anwendungsbereich der Vorschrift. Für sie besteht nach $₫ 17$ Abs. 1 Nr. 4 SGB I die Pflicht, dass Verwaltungs- und Dienstgebäude frei von Zugangs- und Kommunikationsbarrieren sind und Sozialleistungen in barrierefreien Räumen und Anlagen ausgeführt werden (vgl. Welti 2015b). Diese bereits seit 2002 bestehende Hinwirkungspflicht dürfte sich zur Erfüllungspflicht verdichtet haben. Dies dürfte, auch angesichts von Umsetzungsmängeln (Welti u.a. 2014, 190ff.), eine häufige Fallgruppe des Anspruchs auf angemessene Vorkehrungen sein (Welti u.a. 2019, 322).

In anderen Staaten werden Verstöße gegen Barrierefreiheit unmittelbarer in das Antidiskriminierungsrecht einbezogen. So enthält Art. 2 Satz 3 Schweizer BehiG den Tatbestand der Benachteiligung beim Zugang zu einer Baute, einer Anla- 
ge, einer Wohnung oder einer Einrichtung oder einem Fahrzeug des öffentlichen Verkehrs. In Art. 5 Abs. 2 des österreichischen BGStG wird die mittelbare Diskriminierung unter anderem dadurch definiert, dass Merkmale gestalteter Lebensbereiche Menschen mit Behinderungen gegenüber anderen Personen in besonderer Weise benachteiligen können (Buchinger 2013, 96). In den USA wird Barrierefreiheit - teils sehr effektiv - im Wesentlichen als angemessene Vorkehrung betrachtet und eingeklagt (Fuerst 2009, 106; Bowen 2013, 91).

Insgesamt zeigt sich, dass die Zugänglichkeit nach Art. 9 UN-BRK und die Barrierefreiheit nach $\$ 4$ BGG der Beseitigung oder Minimierung sächlicher umweltbedingter Barrieren zugeordnet sind. Die einstellungsbezogenen Barrieren hingegen können durch Bewusstseinsbildung (Art. 8 UN-BRK) und Benachteiligungsverbote verringert werden. Hierbei ist zu fragen, ob die Bezeichnung dieser Barrieren als „einstellungsbezogen“ geeignet ist, das Gemeinte zu treffen. Im englischen Text der Präambel ist von „attitudinal barriers“ die Rede. „Attitude“ kann sowohl mit „Einstellung“ als auch mit „Haltung“ oder „Verhalten“ übersetzt werden. Noch deutlicher ist die Ausrichtung zum Bedeutungsfeld „Verhalten“ im Französischen, wo von „barrières comportementales“ die Rede ist. Rechtliche Regelungen können und sollen im Wesentlichen nur auf behinderndes Verhalten zielen. Zugrundeliegende Einstellungen können nicht geboten oder verboten werden. Sie sind, wie die Psychologie, die den Habitus erforschende Soziologie oder eine ihrer Grenzen bewusste Pädagogik wissen, nur schwer und allmählich zu beeinflussen (vgl. Baer 2017, 241ff.).

\section{Pflicht zur Barrierefreiheit - Recht auf Barrierefreiheit}

\subsection{Barrierefreiheit als objektive Verpflichtung}

Aus der Definition von Barrierefreiheit in $\$ 4$ BGG und den entsprechenden Landesgesetzen folgt noch kein Recht und keine Pflicht. Sie bietet nur den Ausgangspunkt für ein weites Verständnis von Barrieren und Barrierefreiheit in anderen Normen, die auf den Begriff Bezug nehmen, und ermöglicht durch den systematischen Zusammenhang auch, den Abbau von Barrieren zum Gegenstand individuellen Benachteiligungsschutzes zu machen.

Aus Art. 9 UN-BRK folgen hingegen Pflichten der Vertragsstaaten zu geeigneten Maßnahmen, welche die Feststellung und Beseitigung von Zugangshindernissen und -barrieren einschließen. Die Konkretisierung der Staatenpflichten in Art. 9 Abs. 2 UN-BRK umfasst ausdrücklich auch sicherzustellen, dass private Rechtsträger, die Einrichtungen und Dienste für die Öffentlichkeit anbieten, alle Aspekte der Zugänglichkeit berücksichtigen (Art. 9 Abs. 2 lit. b UN-BRK). In der Denkschrift zur Ratifizierung der UN-BRK bezeichnete die Bundesregierung Barrierefreiheit als Zielvorgabe für die Gestaltung aller Lebensbereiche (BT-Drs. 16/10808, 51). Da- 
mit wird sie als verbindliches Prinzip gekennzeichnet, das bei der konventionskonformen und völkerrechtsfreundlichen Anwendung und Auslegung des Rechts auf allen Ebenen zu berücksichtigen ist (zur Wirkung der UN-BRK: Welti 2016).

Feste Regeln ergeben sich erst aus weiteren Normen, die aktuell insbesondere im öffentlichen Recht verortet sind. Sie treffen zunächst die Behörden selbst, die insbesondere zur Barrierefreiheit ihrer Bauten und Anlagen ( $\$ 8$ BGG), Bescheide und Vordrucke ( $\$ 10$ BGG) und Informationstechnik ( $\$ 12$ BGG) (Carstens 2018) verpflichtet sind (für alle relevanten Landesgesetze vgl. Frehe \& Welti 2018). Zur Gestaltung des öffentlichen Raums relevant sind zunächst Planungsnormen etwa des Bauplanungsrechts ( $\$ 1$ Abs. 6 Nr. 3 Baugesetzbuch - BauGB), des Fernstraßenrechts ( $\$ 3$ Abs. 1 Satz 2 Fernstraßengesetz - FStrG) und des landesrechtlichen Straßenrechts, des Eisenbahnrechts ( $\$ 2$ Abs. 3 EisenbahnBau- und Betriebsordnung - EBBO) (Fuerst 2018) und des Nahverkehrsrechts der Länder. Barrierefreiheit ist insoweit Präventionsrecht für Behinderungen und Benachteiligungen.

Für Private und Unternehmen wird Barrierefreiheit über ordnungsrechtliche Normen vermittelt, die Barrieren als zu verhindernde abstrakte Gefahr behandeln, insbesondere im Bauordnungsrecht der Länder, im öffentlichen Arbeitsschutzrecht ( $\$ 3$ a Abs. 2 Arbeitsstättenverordnung - ArbStättV) (Frankenstein 2018) oder im Gaststättenrecht der Länder (Jürgens 2008). Diese Normen sind durch Behörden durchzusetzen, so dass bei ihrer Durchsetzung zunächst die politische Kontrolle durch Regierungen und Parlamente, Aufsichtsbehörden und Selbstverwaltungsorgane relevant ist. Dies verweist auch darauf, wie stark und wirksam dort die Partizipation von Menschen mit Behinderungen ausgeprägt ist.

\subsection{Barrierefreiheit als subjektives Recht}

Tendenziell bleibt die Durchsetzung objektiven Rechts defizitär, gerade wenn sie die Interessen von Minderheiten betrifft, die zudem schwachen Zugang zu ökonomischen Ressourcen und Bildung haben. Die Rechtsmobilisierung bei Menschen mit Behinderungen hat ihrerseits Barrieren, die weiterer Forschung bedürfen (Kocher 2013; Rambausek 2017, 408). Als Ergänzung der politischen und administrativen Kontrolle kann eine rechtliche Kontrolle objektiver Entscheidungen dienen, für die im Bundesrecht ( $\$ 15$ BGG) und Landesrecht Verbandsklagerechte der anerkannten Verbände von Menschen mit Behinderungen vorgesehen sind. Verbandsklagerechte haben im deutschen Verbraucherschutzrecht (Höland 2013) und Umweltrecht (Schlacke 2013) sowie insgesamt in vielen Rechtsordnungen für die Durchsetzung kollektiver Interessen (Halfmeier 2013) eine erhebliche Bedeutung entwickeln können. Ihre Nutzung im deutschen Behindertenrecht ist aber nach wie vor schwach (Welti u.a. 2014, 482ff.), möglicherweise, weil die deutschen Behindertenverbände ihre Stärke traditionell eher im Rechtsschutz zur Unterstützung von Individualklagen haben. 
Rechtsdogmatisch ist relevant, welche Möglichkeit Menschen mit Behinderungen haben, die Beseitigung von Barrieren rechtlich einzufordern, so dass sich Barrierefreiheit als subjektives Recht darstellt. Die Beseitigung von Barrieren, die Menschen mit Behinderungen unmittelbar beeinträchtigen, ist ein Recht auf angemessene Vorkehrungen. Die Vertragsstaaten müssen alle geeigneten Schritte unternehmen, um die Bereitstellung angemessener Vorkehrungen zu gewährleisten. Insoweit kann jedes rechtliche Benachteiligungsverbot im Sinne angemessener Vorkehrungen ausgelegt werden. Eine ausdrückliche Normierung ist jedoch aus Gründen der Klarstellung und der Effektivierung des Rechts angezeigt. Gegenüber Bundesbehörden und Landes- und Kommunalbehörden, die Bundesrecht ausführen, ist dies 2016 in $\$ 7$ Abs. 2 BGG geschehen. In den Behindertengleichstellungsgesetzen der Länder sind entsprechende Anpassungen teilweise bereits erfolgt. Im Zivilrecht fehlt es für das allgemeine Benachteiligungsverbot wegen einer Behinderung in $\$ 1$ AGG bislang an einer Normierung der Pflicht zu angemessenen Vorkehrungen, so dass ungeklärt ist, wie weit subjektive Rechte auf angemessene Vorkehrungen gegen Anbieter von Waren und Dienstleistungen auf $\$ 19$ AGG gestützt werden können. Eine klarstellende Normierung wird daher zu Recht gefordert (Eichenhofer 2019). Wo besondere Regeln gelten, kann der Abbau von Barrieren auch im Rahmen bestehender Vertragsbeziehungen gefordert werden. Dies gilt für die Barrierefreiheit des Arbeitsplatzes nach $₫ 3 \mathrm{a}$ Abs. 2 ArbStättV im Rahmen der Arbeitgeberpflichten nach $\$ 618$ BGB (Frankenstein 2018) und für die barrierefreie Zugänglichkeit der Mietwohnung nach $\$ 554$ a BGB.

Subjektive Rechte Einzelner auf die Beseitigung von Barrieren finden ihre Grenze in der persönlichen Betroffenheit und in der Angemessenheit. Werden sie auf das Gebot angemessener Vorkehrungen gestützt, so können sie möglicherweise durch Assistenz oder organisatorische Maßnahmen erfüllt werden, auch wenn Menschen mit Behinderungen eine barrierefreie Gestaltung von Umwelten vorziehen würden. Andererseits kann hergestellte Barrierefreiheit auch subjektive Rechte auf Assistenz und Unterstützung entfallen lassen.

\section{Wie bestimmt man rechtlich gebotene Barrierefreiheit?}

\subsection{Barrierefreiheit als zu bestimmender Rechtsbegriff}

Barrierefreiheit im Sinne von $\$ 4$ BGG ist ein Rechtsbegriff, der behördlich und gerichtlich erkennbar und überprüfbar ist. Die Frage, ob eine Umwelt barrierefrei gestaltet ist und wird, steht also nicht im behördlichen oder gerichtlichen Ermessen. Ein Ermessen kann allenfalls in Planungsprozessen etwa des Bauplanungsrechts oder des Denkmalschutzrechts bestehen und anerkannt werden, wenn der Belang Barrierefreiheit gegen andere öffentliche und private Belange abzuwägen ist. 


\subsection{Empirie und Normativität}

Der Begriff der Barrierefreiheit einer gestalteten Umwelt nach $\$ 4$ BGG enthält eine Prognoseentscheidung, dass diese Umwelt voraussichtlich keine Barriere für einen Menschen mit gesundheitlicher Beeinträchtigung darstellen wird. Diese Prognose setzt eine Generalisierung dazu voraus, welche gesundheitlichen Beeinträchtigungen mit welchen Umweltbedingungen wie in Wechselwirkung treten und sich dabei als Barrieren auswirken können. Im General Comment No 2 (Nr. 25) wird davon gesprochen, dass Standards breit und standardisiert sein müssen. Für Personen mit seltenen Beeinträchtigungen oder solchen, die nicht die Modalitäten, Methoden oder Mittel nutzen, die angeboten werden, um $\mathrm{Zu}$ gänglichkeit herzustellen - z.B. die nicht Braille lesen können - können auch Standards nicht hinreichend sein, so der GC No 2 weiter.

Die Standards für eine möglichst gute Prognose können sich aus einer Zusammenschau einschlägiger wissenschaftlicher Erkenntnisse insbesondere der Medizin, Psychologie, Rehabilitationswissenschaften, Architektur und Ingenieurwissenschaften ergeben. Die in $\$ 8$ Abs. 1 Satz 1 BGG genannten allgemein anerkannten Regeln der Technik beziehen sich insoweit nicht auf eine bestimmte Wissenschaft, sondern auf alle verallgemeinerbaren Erkenntnisse, die notwendig sind, um die Vermeidbarkeit von Barrieren theoretisch und praktisch zu beurteilen. Häufig wird hierzu in Deutschland auf Regelwerke des Deutschen Instituts für Normung (DIN) zurückgegriffen. Ob diese ein für die rechtliche Beurteilung geeignetes und hinreichendes Instrument sind, kann im Einzelfall bezweifelt und überprüft werden.

Aus empirisch zu ermittelnden oder auf fachlicher Basis zu antizipierenden Erkenntnissen kann ermittelt werden, ob gestaltete Lebensbereiche für Menschen mit Beeinträchtigungen in der allgemein üblichen Weise ohne fremde Hilfe auffindbar, zugänglich und nutzbar sind. Schon beim Element des allgemein Üblichen wird jedoch zu beachten sein, dass dessen Bestimmung zwar empirisch möglich ist, in die Vorannahmen jedoch auch Wertungen eingehen können. Ob eine „allgemeine“ Üblichkeit gegeben ist, wenn mehr als die Hälfte, drei Viertel oder fast alle Nutzerinnen und Nutzer einen bestimmten Gebrauch von etwas machen, sagt das Gesetz nicht, kann aber entscheidend für die Freiheitsgrade sein, die in der Gestaltung von Lebenswelten ermöglicht werden. Normativ auszufüllen sind auch die Fragen, wann eine Erschwernis „besonders“ ist und welche Art von fremder Hilfe im Einzelfall zumutbar ist, wenn sie nur "grundsätzlich“ entbehrlich sein soll. Um diese unbestimmten Rechtsbegriffe wertend auszufüllen, ist auf die Ziele des Gesetzes zurückzugreifen, also nach $₫ 1$ Abs. 1 Satz 1 BGG das Beseitigen und Verhindern der Benachteiligung von Menschen mit Behinderungen, die gleichberechtigte Teilhabe am Leben in der Gesellschaft und die Ermöglichung einer selbstbestimmten Lebensführung. Die UN-BRK bietet für einzelne gestaltete Lebensbereiche viele weitere und konkretere Anhaltspunkte für die gebotene Zugänglichkeit. 


\subsection{Barrierefreiheit und Partizipation}

Die Komplexität und Wertungsbedürftigkeit der mit dem Rechtsbegriff der Barrierefreiheit verbundenen Entscheidungen legt nahe, dass diese in einem geregelten kooperativen Verfahren zwischen Staat und Gesellschaft unter Einbeziehung der Wissenschaft getroffen werden. Dies lässt sich auch in anderen Rechtsbereichen mit vergleichbaren Begriffen beobachten wie der menschengerechten Gestaltung der Arbeit ( $\$ 2$ Abs. 1 Arbeitsschutzgesetz - ArbSchG), den Anforderungen an gesunde Wohn- und Arbeitsverhältnisse ( $\$ 34$ Abs. 1 Satz 2 Baugesetzbuch - BauGB) oder die ausreichenden, zweckmäßigen und wirtschaftlichen Leistungen der gesetzlichen Krankenversicherung ( $\$ 12$ Abs. 1 Sozialgesetzbuch Fünftes Buch - SGB V). Um diese Rechtsbegriffe zu operationalisieren werden nicht nur behördliche und wissenschaftliche Sachkenntnis, sondern über die kommunale und soziale Selbstverwaltung sowie die betriebliche Mitbestimmung auch die organisierte und legitimierte Interessenvertretung betroffener Gruppen einbezogen.

Eine entsprechende Ausfüllung und Legitimation von Barrierefreiheit durch die Beteiligung von Menschen mit Behinderungen liegt nahe. Art. 4 Abs. 3 UN-BRK fordert von den Vertragsstaaten, Menschen mit Behinderungen durch die sie vertretenden Organisationen aktiv einzubeziehen. Das Behindertengleichstellungsrecht sieht inzwischen mit der Bundesfachstelle für Barrierefreiheit ( $\$ 13$ BGG) eine institutionelle Schnittstelle von Behörden, Verbänden und Wissenschaft vor. Auch der Beauftragte der Bundesregierung für die Belange von Menschen mit Behinderungen ( $\$ 17$ BGG) und die Beauftragten in Ländern und Kommunen organisieren die Kooperation von Gesetzgebung, Regierung, Verwaltung und Verbänden. Gerade auf der kommunalen Ebene muss Barrierefreiheit zur Realisierung der gemeindenahen Unterstützung Gegenstand partizipativer Teilhabeplanung werden (vgl. Welti 2011; Rambausek 2017, 299).

In den Betrieben ist die Schwerbehindertenvertretung ( $\$ 178$ SGB IX) ein wichtiges Medium der Partizipation. Sie kann in Inklusionsvereinbarungen mit den Arbeitgebern ( $\$ 166$ SGB IX) auch Regelungen zur Arbeitsplatzgestaltung vereinbaren (Welti \& Groskreutz 2016). Auch in Schulen und in Hochschulen (Welti 2019) bedarf es interner partizipativer Strukturen. Entscheidende Faktoren für eine gelingende partizipative Konkretisierung von Barrierefreiheit ist, dass die relevanten Akteure beteiligt werden und die rechtliche und institutionelle Ausgestaltung Druck für kooperative Entscheidungen schafft. Akteure müssen Zugang $\mathrm{zu}$ fachlicher und rechtlicher Beratung haben und die Ergebnisse müssen vorgerichtlich und gerichtlich überprüfbar sein.

\section{Schluss}

Wegen der bislang sehr überschaubaren Menge von Judikaten ist Barrierefreiheit bislang kein Thema einer intensiven rechtswissenschaftlichen Diskussion gewe- 
sen. Es wäre erfreulich, wenn sich dies ändern würde. Barrieren für gesundheitlich beeinträchtigte Menschen sind gesellschaftlich relevant. Der Umgang mit ihnen enthält interessante Rechtsfragen, die an der Schnittstelle von Antidiskriminierungsrecht, Ordnungsrecht, Planungsrecht und vielen je spezifischen Rechtsgebieten liegen. Es ist zu erwarten, dass das EU-Recht in den nächsten Jahren die Diskussion beleben wird.

\section{Literatur}

Alexy, R. (1994): Theorie der Grundrechte. Frankfurt/M.: Suhrkamp.

Baer, S. (2017): Rechtssoziologie - Eine Einführung in die interdisziplinäre Rechtsforschung. 3. Aufl. Baden-Baden: Nomos.

Banafsche, M. (2015): Die internationalen Menschenrechte und das deutsche Recht. In: M. Banafsche \& H.-W. Platzer (Hrsg.): Soziale Menschenrechte und Arbeit - Multidisziplinäre Perspektiven. Baden-Baden: Nomos, 57-88.

Bösl, E. (2012): Behinderung, Technik und gebaute Umwelt. Zur Geschichte des Barriereabbaus in der Bundesrepublik Deutschland seit dem Ende der 1960er Jahre. In: A. Tervooren \& J. Weber (Hrsg.): Wege zur Kultur - Barrieren und Barrierefreiheit in Kultur- und Bildungsreinrichtungen. Köln, Weimar und Wien: Böhlau.

Bösl, E. (2016): Bundesdeutsche Behindertenpolitik im „Jahrzehnt der Rehabilitation“ - Umbrüche und Kontinuitäten um 1970. In: G. Lingelbach \& A. Waldschmidt (Hrsg.): Kontinuitäten, Zäsuren, Brüche? - Lebenslagen von Menschen mit Behinderungen in der deutschen Zeitgeschichte. Frankfurt/M. und New York: Campus, 82-115.

Bowen, I. (2013): The American Experience: What makes ADA work and what are the Implications for Implementation of Germany's Accessibility Laws? In: F. Welti (Hrsg.): Rechtliche Instrumente zur Durchsetzung von Barrierefreiheit. Kassel: Kassel University Press, 79-94.

Buchinger, E. (2013): Rechtliche Instrumente zur Durchsetzung von Barrierefreiheit: Erfahrungen aus Österreich. In: F. Welti (Hrsg.): Rechtliche Instrumente zur Durchsetzung von Barrierefreiheit. Kassel: Kassel University Press, 2013, 95-98.

Carstens, A. (2018): Barrierefreie Informationstechnik. In: O. Deinert \& F. Welti (Hrsg.): Stichwortkommentar Behindertenrecht. 2. Aufl. Baden-Baden: Nomos, 115-124.

Degener, T. (2019): Die UN-Behindertenrechtskonvention - Ansatz einer inklusiven Menschenrechtstheorie. In: S. Baer, O. Lepsius, C. Schönberger, C. Waldhoff \& C. Walter (Hrsg.): Jahrbuch des Öffentlichen Rechts der Gegenwart - Neue Folge, Bd. 67. Tübingen: Mohr Siebeck, 487-508.

Eichenhofer, E. (2018): Angemessene Vorkehrungen als Diskriminierungsdimension im Recht Menschenrechtliche Forderungen an das Allgemeine Gleichbehandlungsgesetz. Berlin: Antidiskriminierungsstelle des Bundes.

Frankenstein, A. (2018): Universelles Design und Zugänglichkeit der Arbeitsplätze. In: G. Wansing, F. Welti \& M. Schäfers (Hrsg.): Das Recht auf Arbeit für Menschen mit Behinderungen Internationale Perspektiven. Baden-Baden: Nomos, 227-246.

Frehe, H. (2013): Das Gleichstellungsgesetz für Menschen mit Behinderung. In: F. Welti (Hrsg.): Rechtliche Instrumente zur Durchsetzung von Barrierefreiheit. Kassel: Kassel University Press, 17-22.

Frehe, H. \& Welti, F. (2018): Behindertengleichstellungsrecht - Textsammlung mit Einführungen. 3. Aufl. Baden-Baden: Nomos.

Fuerst, A.-M. (2009): Behinderung zwischen Diskriminierungsschutz und Rehabilitationsrecht - Ein Vergleich zwischen Deutschland und den USA. Baden-Baden: Nomos.

Fuerst, A.-M. (2018): Öffentlicher Personenfernverkehr. In: O. Deinert \& F. Welti (Hrsg.): Stichwortkommentar Behindertenrecht. 2. Aufl. Baden-Baden: Nomos, 837-842. 
Halfmeier, A. (2013): Begriffe und Perspektiven des Verbandsklagerechts. In: F. Welti (Hrsg.): Rechtliche Instrumente zur Durchsetzung von Barrierefreiheit. Kassel: Kassel University Press, 125-135.

Heyer, K. (2015): Rights Enabled - The Disability Revolution, from the US, to Germany and Japan, to the United Nations. Ann Arbor: University of Michigan Press.

Hirschberg, M. (2009): Behinderung im internationalen Diskurs. Die flexible Klassifizierung der Weltgesundheitsorganisation. Frankfurt/M. und New York: Campus.

Hlava, D. (2018): Barrierefreie Gesundheitsversorgung - Rechtliche Gewährleistung unter besonderer Berücksichtigung der Rechtsdurchsetzung. Baden-Baden: Nomos.

Höland, A. (2013): Verbandsklagen im Verbraucherrecht und im Sozialrecht - vergleichende Überlegungen. In: F. Welti (Hrsg.): Rechtliche Instrumente zur Durchsetzung von Barrierefreiheit. Kassel: Kassel University Press, 113-124.

Jürgens, U (2008): Barriere- und diskriminierungsfreier Zugang zu öffentlichen Gaststätten. Frankfurt/M.: Peter Lang.

Kocher, E. (2013): Barrieren der Rechtsmobilisierung. In: F. Welti (Hrsg.): Rechtliche Instrumente zur Durchsetzung von Barrierefreiheit. Kassel: Kassel University Press, 73-78.

Kuhn-Zuber, G. (2018): Flugverkehr. In: O. Deinert \& F. Welti (Hrsg.): Stichwortkommentar Behindertenrecht. 2. Aufl. Baden-Baden: Nomos, 399-401.

Rambausek, T. (2017): Behinderte Rechtsmobilisierung - Eine rechtssoziologische Untersuchung zur Umsetzung von Artikel 19 der UN-Behindertenrechtskonvention. Wiesbaden: Springer VS.

Schaumberg, T. (2018): Universelles Design. In: O. Deinert \& F. Welti (Hrsg.): Stichwortkommentar Behindertenrecht. 2. Aufl. Baden-Baden: Nomos, 1127-1132.

Schlacke, S. (2013): Verbandsklagen im Umwelt- und Verwaltungsrecht. In: F. Welti (Hrsg.): Rechtliche Instrumente zur Durchsetzung von Barrierefreiheit. Kassel: Kassel University Press, 99-112.

Theben, M. (2018): Barrierefreiheit. In: O. Deinert \& F. Welti (Hrsg.): Stichwortkommentar Behindertenrecht. 2. Aufl. Baden-Baden: Nomos, 124-132.

Welti, F., Frankenstein, A. \& Hlava, D. (2019): Angemessene Vorkehrungen und Sozialrecht. In: Die Sozialgerichtsbarkeit (6), 317-325.

Welti, F. \& Groskreutz, H. (2016): Betriebliche Barrierefreiheit als Aufgabe der Schwerbehindertenvertretung. In: Arbeit und Recht (AuR) (3), 105-108.

Welti, F., Groskreutz, H., Hlava, D., Rambausek, T., Ramm, D. \& Wenckebach, J. (2014): Evaluation des Behindertengleichstellungsgesetzes im Auftrag des Bundesministeriums für Arbeit und Soziales. BMAS-Forschungsbericht 445. Bonn: BMAS.

Welti, F. (2019): Rechtlicher Rahmen für ein barrierefreies System Hochschule. In: P. Tolle, A. Plümmer \& A. Horbach (Hrsg.): Hochschule als interdisziplinäres barrierefreies System. Kassel: Kassel University Press, 33-41.

Welti, F. (2016): Potenzial und Grenzen der menschenrechtskonformen Auslegung des Sozialrechts am Beispiel der UN-BRK. In: U. Faber, K. Feldhoff, K. Nebe, K. Schmidt \& U. Waßer (Hrsg.): Gesellschaftliche Bewegungen - Recht unter Beobachtung und in Aktion - Festschrift für Wolfhard Kohte. Baden-Baden: Nomos, 635-658.

Welti, F. (2015a): Soziale Menschenrechte in Wissenschaft und Praxis. In: M. Banafsche \& H.-W. Platzer (Hrsg.): Soziale Menschenrechte und Arbeit - Multidisziplinäre Perspektiven. Baden-Baden: Nomos, 17-31.

Welti, F. (2015b): Barrierefreiheit und das Sozialrecht. In: W. Kohte \& N. Absenger (Hrsg.): Menschenrechte und Solidarität im internationalen Diskurs - Festschrift für Armin Höland. BadenBaden: Nomos, 245-258.

Welti, F. (2012): Artikel 9 - Zugänglichkeit. In: A. Welke (Hrsg.): UN-Behindertenrechtskonvention mit rechtlichen Erläuterungen. Berlin: Deutscher Verein für öffentliche und private Fürsorge, 127-135.

Welti, F. (2011): Rechtliche Grundlagen einer örtlichen Teilhabeplanung. In: D. Lampke, A. Rohrmann \& J. Schädler (Hrsg.): Örtliche Teilhabeplanung mit und für Menschen mit Behinderungen. Wiesbaden: VS, 55-68. 


\section{Barrieren als gesellschaftliche Hindernisse - Sozialwissenschaftliche Überlegungen}

Barrierefreiheit und auch das übergeordnete Prinzip der Zugänglichkeit sind am Verhältnis von Individuum und Gesellschaft ausgerichtet, also konkret an den individuellen Lebensmöglichkeiten innerhalb der jeweiligen gesellschaftlichen Bedingungen unter Beachtung von Zeit und Ort. Hiermit ist die Frage nach Barrieren gestellt, die einzelne Menschen und auch Gruppen von Menschen in ihrer Teilhabe an der Gesellschaft in unterschiedlicher Weise behindern. Welche Barrieren bestehen wo für wen? Wie lassen sich diese konkret erfassen und abbauen? Im Folgenden werden unterschiedliche theoretische Herangehensweisen - ausgehend entweder vom Individuum oder von räumlichen Bedingungen - dargestellt, aus Perspektive der Disability Studies diskutiert und hinsichtlich ihrer Bedeutung für die Verringerung gesellschaftlicher Hindernisse reflektiert.

\section{Relevanz von Barrierefreiheit für die Teilhabe aller Menschen an der Gesellschaft}

Barrieren sind nicht an sich bedeutend, sondern nur als etwas, das für alle - und spezifisch für bestimmte Gruppen behinderter Menschen - abzubauen ist. Ziel ist es, sie zu erkennen und so zu verringern oder vollständig aufzulösen, dass Menschen mit unterschiedlichen Beeinträchtigungen an der Gesellschaft teilhaben können.

Begreift man Behinderung nicht als Thema einiger, sondern als gesellschaftliches Thema, dann betrifft es alle, auch in Form intersektionaler Benachteiligung behinderter Menschen (vgl. Charlton 2006; Wansing \& Westphal 2014 und 2018). Generell existieren Krankheiten, chronische Erkrankungen und Behinderungen nicht für sich allein oder als ausschließlich individuelles Merkmal, sondern nur in Verbindung mit Menschen in ihrer jeweiligen Umwelt, in unterschiedlichen kulturellen Kontexten und Gesellschaften (vgl. Siegrist 2002). Darüber hinaus variiert es, sich krank zu fühlen, mit Gender, Klasse, Ethnizität, Alter und Religion sowie mit weniger offensichtlichen Faktoren wie der Unterstützung von Familienmitgliedern oder Freunden (vgl. Hall 1980; Leach Scully 2004; Wengler 2012). Daher ist von einer individuellen Verkörperung des gesellschaftlichen Phänomens Behinde- 
rung zu sprechen: (Chronische) Erkrankungen und Behinderungen sind, ebenso wie Gesundheit und Funktionsfähigkeit, jeweils individuell verkörpert, was auch für den Gesundheitszustand der Gesellschaft bedeutsam ist (vgl. Bourdieu 1987; Gugutzer 2012). Hierbei ist auch zu beachten, dass alle Menschen innerhalb ihres Lebens eine (chronische) Erkrankung oder Beeinträchtigung erwerben können (vgl. WHO \& World Bank 2011). Menschen sind somit nur zeitweilig nichtbehindert, wofür in den Disability Studies der Terminus „temporarily or momentarily able-bodied" geprägt worden ist (Zola 1993, 171). Aus dieser Perspektive sind Beeinträchtigungen keine Ausnahme menschlicher Existenz, sondern üblich, sie können als das Reguläre verstanden werden (vgl. Hirschberg \& Valentin 2020). Damit ist es entscheidend, dass Menschen mit Beeinträchtigungen nicht Barrieren begegnen und durch diese nicht behindert werden (Behinderungsbegriff nach Art. 1 Satz 2 UN-BRK).

Barrieren sind daher für alle Mitglieder der Gesellschaft relevant. Ihre Bedeutung schärft sich mit dem Ziel der Barrierefreiheit, was für physische Barrieren einfach, für einstellungsbedingte Barrieren komplexer nachzuvollziehen ist, weil sich der Abbau von Vorurteilen und negativen Einstellungen gegenüber behinderten Menschen anders gestaltet (vgl. Campbell 2009; Wiedemann \& Roßberg 2016).

\section{Menschenrechte als Analyse-Instrument und normative Grundlage für Politik}

Mit der seit dem 26. März 2009 in Deutschland rechtsverbindlich in Kraft getretenen UN-Behindertenrechtskonvention (UN-BRK) ist der Staat verpflichtet, „den vollen und gleichberechtigten Genuss aller Menschenrechte und Grundfreiheiten durch alle Menschen mit Behinderungen zu fördern, zu schützen und zu gewährleisten und die Achtung der ihnen innewohnenden Würde zu fördern“ (Art. 1 UAbs. 1 BRK). Zu dieser staatlichen Verpflichtung gehören viele Aufgaben, die aus den Erfahrungen behinderter Menschen resultieren und nun in der UN-BRK in Form mehrerer Grundsätze und Menschenrechtsprinzipien sowie Einzelrechten aufgenommen sind. So sind Barrierefreiheit, Chancengleichheit, Inklusion und Nicht-Diskriminierung als Menschenrechts-Grundsätze ausdrücklich genannt (Art. 3 f, e, c, b UN-BRK), Art. 9 UN-BRK führt aus, was unter Zugänglichkeit (Barrierefreiheit gemäß der Schattenübersetzung der UN-BRK, vgl. Netzwerk Artikel 3 2018) in umfangreichem Maß zu verstehen ist.

Menschenrechtsverträge sind als Antwort auf strukturelle Unrechtserfahrungen zu verstehen, die einzelne oder Gruppen von Menschen gemacht haben. Menschenrechte sind nicht selbstverständlich allen gegeben, sondern wurden - wie bei den gruppenbezogenen Menschenrechtsverträgen deutlich wird - gefordert, erkämpft und schließlich von vielen Akteur*innen unterschiedlicher Pro- 
fessionen, den Selbstvertretungsorganisationen behinderter Menschen und den Staatenvertreter*innen bei den Vereinten Nationen verhandelt. Diese Entwicklung wird auch an anderen Menschenrechtsverträgen deutlich. Verallgemeinert formuliert, wurden mit den Menschenrechtsabkommen Anliegen von Befreiungsoder sozialen Bewegungen aufgegriffen, wie z.B. der Arbeiter- oder der Frauenbewegung, des Civil Rights Movement der Afroamerikaner*innen und anderer Antirassismusbewegungen, der Bewegungen für die Rechte von Kindern, des Queer Movements sowie der Behindertenbewegungen unterschiedlicher Länder.

Die Beachtung und Einhaltung der allgemeinen Menschenrechte für behinderte Menschen wurde auch bereits bei der „Bühnenbesetzung der Dortmunder Westfalenhalle zum UNO-Jahr" 1981 gefordert, als Ausgrenzung kritisiert und Menschenrechtsverletzungen angeprangert wurden: „Keine Reden, keine Aussonderung und keine Menschenrechtsverletzungen!" (Köbsell 2012, 14f.). Mit diesen Forderungen wurde Partizipation als gesellschaftliche Aufgabe begriffen, was mit der Behindertenrechtskonvention aufgenommen wurde.

Die Äußerungen des Fachausschusses zur UN-BRK bei den Vereinten Nationen, der für das Monitoring des vertragsstaatlichen Handeln verantwortlich ist und die Aufgabe hat, die Auslegung der Konventionsvorschriften zu vereinheitlichen, können dabei als Handlungsleitlinien auch über den juristischen Gebrauch hinaus verwendet werden, um die gesellschaftliche Partizipation behinderter Menschen zu analysieren: Welche Rechte sind umgesetzt und bestehen damit nicht nur passiv, sondern können aktiv ausgeübt und damit gelebt werden? Welche Anforderungen müssen an die Umsetzung gestellt werden und wer ist mit ihr beauftragt?

\section{Bestimmung des Begriffs Barrieren}

Derzeit gibt es mehrere begriffliche Konstruktionen von Barrieren, die relevant sind: im Bundes- und in den Landesbehindertengleichstellungsgesetzen, in der Internationalen Klassifikation von Funktionsfähigkeit, Behinderung und Gesundheit (ICF) der Weltgesundheitsorganisation sowie in der UN-Behindertenrechtskonvention. Mit diesen sind Systeme verbunden, die Infrastruktur, Gegenstände oder auch Hilfsmittel näher erfassen: die Deutsche Industrienorm (DIN), z.B. zur Bestimmung von Türbreiten für die Durchfahrt mit dem Rollstuhl, oder auch der Katalog der Internationalen Standardisierungsorganisation zur Klassifizierung von Hilfsmitteln (ISO 9999).

Zur konkreteren Diskussion wird im Folgenden erklärt, wie Barrieren und Barrierefreiheit bzw. Zugänglichkeit definiert werden, um anschließend auf Erhebungsmöglichkeiten von Barrieren näher einzugehen. 


\subsection{Barrieren und Barrierefreiheit im Antidiskriminierungsrecht}

Die 1994 vorgenommene Ergänzung des Gleichheitssatzes im Grundgesetz um das Diskriminierungsverbot behinderter Menschen „Niemand darf wegen seiner Behinderung benachteiligt werden" ist besonders bedeutsam, weil mit diesem Gleichheitssatz hervorgehoben wird, dass behinderte Menschen Grundrechtsträger*innen sind (Art. 3 Abs. 3 Satz 2 GG; vgl. Degener 2003, 460). Kein Gesetz darf diesem Grundsatz widersprechen. Sofern dieser Diskriminierungsschutz nicht erfüllt wird, besteht sowohl für Einzelpersonen als auch für Verbände die Möglichkeit zu klagen.

Im Antidiskriminierungsrecht können folgende Prinzipien unterschieden werden: das formale und das strukturelle Gleichheitsprinzip. Gemäß dem formalen Gleichheitsprinzip werden behinderte und nicht-behinderte Menschen formal als gleich angesehen, was sich u.a. in „separate but equal“-Leistungen ausdrückt. Faktisch erhalten sie jedoch unterschiedliche Leistungen bzw. treffen auf unterschiedliche Realitäten, wie z.B. an getrennten Bildungseinrichtungen oder an dem spezifischen Zugang zu Fahrzeugen der Deutschen Bahn für behinderte Menschen ersichtlich wird.

Im Gegensatz zum Prinzip formaler Gleichheit kann nach dem strukturellen Gleichheitsprinzip Behindertendiskriminierung erfasst werden, die auf strukturellen Benachteiligungen durch Barrieren beruht. Das Prinzip struktureller Gleichheit ist in Deutschland durch das Behindertengleichstellungsgesetz (BGG) und das Allgemeine Gleichbehandlungsgesetz (AGG) mit dem Ziel verankert, Behindertendiskriminierung in Form struktureller „Benachteiligungen durch Barrieren, die im Laufe der Geschichte in fast allen gesellschaftlichen Bereichen errichtet wurden“, zu verhindern bzw. abzubauen (Degener 2003, 459).

Demgegenüber definiert das Sozialgesetzbuch (SGB) IX als Sozialleistungsrecht nicht, „was unter Behindertenbenachteiligung zu verstehen ist, so dass es den Gerichten überlassen bleibt, die Vorschrift im Sinne eines formalen oder strukturellen Gleichheitsprinzips zu interpretieren“" (ebd., 462).

Das Gleichstellungsrecht fokussiert sowohl den Abbau von Barrieren, räumlicher oder kommunikativer Art als auch von gesellschaftlichen Vorurteilen und diskriminierenden Verhaltensweisen gegenüber behinderten Menschen (vgl. Campbell 2009). Auch wenn im BGG der Behinderungsbegriff des SGB IX zugrunde gelegt wird, steht diese Bezugnahme auf das Individuum nicht im Vordergrund - selbst, wenn es juristisch häufig um die Vermittlung subjektiver Rechte geht.

Ähnlich ist es im AGG: Dort wird Behinderung als Grund für Benachteiligungen gefasst $(\$ 1$ AGG). Ebenso wie die anderen Kategorien (Geschlecht, Religion/ Weltanschauung, Alter, sexuelle Identität oder ethnische Herkunft), die Grund einer Benachteiligung sein können, wird die Kategorie Behinderung im AGG nur benannt. Im Gegensatz zum Sozialleistungsrecht erfordert das Gleichstellungsrecht keine negative Klassifizierung von Behinderung. Stattdessen wird Behinde- 
rung als neutrale Kategorie einer gesellschaftlichen Gruppe gefasst, die Diskriminierungen oder Benachteiligungen erfahren kann.

Die dem BGG und auch der UN-BRK gemeinsame Sicht, gesellschaftliche Bedingungen gemäß den Bedarfen aller Gesellschaftsmitglieder und damit auch behinderter Menschen zu gestalten, zeigt sich auch in empirischen Studien, auf deren Basis notwendige Veränderungen an Infrastruktur und physischer Umwelt erfasst werden (vgl. Stark u.a. 2007; Gamache u.a. 2017; siehe Abschnitt 4).

\subsection{Barrieren im Sozialleistungsrecht}

Das Sozialrecht befasst sich auf den ersten Blick nicht mit Barrieren, auch wenn sie mittelbar über die Definition von Menschen mit Behinderungen in $\$ 2$ Abs. 1 SGB IX Wirkung entfalten:

„Menschen mit Behinderungen sind Menschen, die körperliche, seelische, geistige oder Sinnesbeeinträchtigungen haben, die sie in Wechselwirkung mit einstellungsund umweltbedingten Barrieren an der gleichberechtigten Teilhabe an der Gesellschaft mit hoher Wahrscheinlichkeit länger als sechs Monate hindern können. Eine Beeinträchtigung nach Satz 1 liegt vor, wenn der Körper- und Gesundheitszustand von dem für das Lebensalter typischen Zustand abweicht."

Auf diese Definition wird in anderen deutschen Gesetzen vielfach verwiesen, es ist die Grundlage für sozialrechtliche Leistungsansprüche in Bezug auf behinderte Menschen. Erhebungen wie der Teilhabe-Survey, begründet durch die UN-BRK, orientieren sich an diesem Verständnis: am Individuum, das aufgrund seiner Beeinträchtigung Barrieren begegnet, und nicht an den gesellschaftlichen Bedingungen, deren Barrieren die Teilhabe für alle erschweren und daher, nicht nur für beeinträchtigte Menschen, abgebaut werden sollten (vgl. Schröder u.a. 2017). Diese scharfe Trennung aufzulösen, erscheint vor dem Hintergrund der UN-BRK zwingender denn je: Nicht zuletzt das Gebot angemessener Vorkehrungen zeigt, dass viele Regelungen des Sozialleistungsrechts dazu dienen können, Benachteiligungen behinderter Menschen zu vermeiden (vgl. Welti u.a. 2019). Ihnen strukturell zu begegnen, ist demnach geboten.

\subsection{Barrieren und Unterstützungsfaktoren in der ICF und die Weiterentwicklung dieser Kategorien}

In der ICF umfasst die Komponente der Umweltfaktoren sowohl Barrieren als auch Förderfaktoren, je bezogen auf die Lebenssituation eines Individuums. Die Umweltfaktoren werden neben den personenbezogenen Faktoren als Teil des individuellen Kontextes verstanden und „müssen aus der Sicht der Person kodiert werden" (WHO 2005, 218).

Die beiden Teilkomponenten der Umwelt - Barrieren und Förderfaktoren - lassen sich als die entscheidende Neuerung der ICF begreifen (vgl. Hirschberg 2009, 285f.; Hurst 2003). Dennoch sind beide noch zu wenig ausdifferenziert, wie ich bereits früher kritisch analysiert habe (vgl. Hirschberg 2012). Auch in der ICF wurde von 
der WHO auf deren sinnvolle zukünftige Erforschung verwiesen: „Es ist zu hoffen, dass zukünftige Forschung zu einem besseren Verständnis dieser Wechselwirkung führen und möglicherweise die Zweckmäßigkeit eines zweiten Qualifikators für diese Faktoren zeigen wird“ (WHO 2005, 218; WHO 2001, 171).

Daher sollten ein zweites und auch drittes Beurteilungsmerkmal konstruiert werden, um zusätzlich zum Ausmaß (als erstem Beurteilungsmerkmal) auch Häufigkeit und Vermeidbarkeit von Barrieren bzw. zusätzlich zum Ausmaß auch Zuverlässigkeit und Qualität erfassen zu können (vgl. Hirschberg 2012, 23). Folgende Schritte wären erforderlich:

- Differenzierung der 4. Klassifikationsebene der Umweltfaktoren

- Entwicklung weiterer Beurteilungsmerkmale (s.o., analog zu den Körperstrukturen):

- Ausmaß, Häufigkeit und Vermeidbarkeit einer Barriere

- Ausmaß, Zuverlässigkeit und Qualität eines Unterstützungsfaktors

- Erweiterte Ausrichtung der Erhebung von Barrieren und Unterstützungsfaktoren am öffentlichen Raum statt ausschließlich individuumsorientiert

Die Umweltfaktoren müssten so präzisiert werden, dass die ICF nicht nur auf individuelle Behinderungen, sondern auch auf gesellschaftliche Behinderungsfaktoren ausgerichtet mit dem Ziel gleichberechtigter gesellschaftlicher Teilhabe eingesetzt werden kann. Wie Gamache u.a. $(2017,243)$ ausführen, ist die Taxonomie der ICF in ihrer Reichweite begrenzt, sodass eine Fundierung notwendig ist (siehe Abschnitt 4.2).

\section{Wie können Barrieren als gesellschaftliche Hindernisse erhoben werden?}

Durch gesellschaftliche Barrieren sind behinderte Menschen benachteiligt und unterliegen damit Ausschlussprozeduren, die besonders im wirtschaftlichen und sozialen Zusammenspiel wirkmächtig sind (vgl. Kronauer 2010). Aus Sicht der Disability Studies ist es unzweifelhaft, dass es die Barrieren und die gesellschaftlichen Benachteiligungen sind, die es zu entfernen gilt, wie in den Prinzipien der Union of the Physically Impaired against Segregation (UPIAS) auf den Punkt gebracht:

„In our view, it is society which disables physically impaired people. Disability is something imposed on top of our impairments, by the way we are unnecessarily isolated and excluded from full participation in society. Disabled people are therefore an oppressed group in society. It follows from this analysis that having low incomes, for example, is only one aspect of our oppression. It is a consequence of our isolation and segregation, in every area of life, such as education, work, mobility, housing, etc. Poverty is one symptom of our oppression, but it is not the cause." (UPIAS 1975, 3f.) 
Diese Positionierung bildet das Fundament des Sozialen Modells von Behinderung, das von der britischen Behindertenbewegung entwickelt wurde. Mike Oliver, der häufig als Vater des Sozialen Modells bezeichnet wird, fasst als einer der britischen Begründer*innen der Disability Studies zusammen, wie Behinderung in den letzten 100 Jahren konstruiert wurde: zum einen als medizinisches Problem, das möglichst geheilt oder gelindert, und zum anderen als soziales Problem, das sozial versorgt werden soll:

„In the past 100 years or so, industrial societies have produced disability first as a medical problem requiring medical intervention and second as a social problem requiring social provision. Research, on the whole, has operated within these frameworks and sought to classify, clarify, map and measure their dimensions" (Oliver 1992, 101).

Die von Oliver kritisierte Perspektive auf behinderte Menschen wurde jedoch ergänzt um eine soziale bzw. menschenrechtsbasierte Forschungsperspektive (vgl. Hirschberg 2014).

Doch nicht erst mit Entwicklung der UN-BRK seit 2001 und ihrer Verabschiedung durch die Vereinten Nationen Ende 2006, sondern bereits nach den beiden Weltkriegen wurden statt der Beeinträchtigung die gesellschaftlichen Hindernisse in den Mittelpunkt gestellt. Das Bestreben der Wiedereingliederung nach dem zweiten Weltkrieg und die Behindertenrechtsbewegung der 1960er und 1970er Jahre als treibende Kraft in den USA sind Faktoren, die zur Entwicklung des Konzepts des Universellen Designs geführt haben (vgl. Paul 2010; Frankenstein 2018, 229). Dieses lässt sich als Einflussfaktor auf das BGG (2002) erkennen, Barrierefreiheit umfänglich als gesellschaftliches Konzept zu verstehen (siehe Abschnitt 3.1). Des Weiteren hat Universelles Design in die UN-BRK Eingang gefunden und dadurch die Orientierung an einem weiten Verständnis von Barrierefreiheit vertieft.

\subsection{Universelles Design: Anforderungen an barrierefreie Orte und Gegebenheiten}

Universelles Design ist ein interdisziplinäres Konzept, das am „Center for Universal Design" an der North Carolina State University mit sieben Prinzipien Mitte bis Ende der 1990er Jahre entwickelt worden ist (vgl. Story 2001; Fisseler 2015). In diesen Prinzipien wird ausgeführt, wie Produkte, Gebäude und der öffentliche Raum möglichst für alle und möglichst weitgehend genutzt werden können. Im Gegensatz zum Prinzip der Barrierefreiheit richtet sich das Konzept des Universellen Designs an alle Menschen, was alle grundsätzlich ein- und niemanden ausschließt. In der UN-BRK wird dieses Konzept aufgegriffen und definiert (Art. 2 UAbs. 5 UN-BRK). Entscheidend ist, dass Universelles Design auch in den allgemeinen Verpflichtungen der Vertragsstaaten, der sog. Implementierungsklausel, explizit genannt ist: Die Vertragsstaaten verpflichten sich, zum Zweck der Menschenrechtsverwirklichung ohne Diskriminierung 
„Forschung und Entwicklung für Güter, Dienstleistungen, Geräte und Einrichtungen in universellem Design, [...], die den besonderen Bedürfnissen von Menschen mit Behinderungen mit möglichst geringem Anpassungs- und Kostenaufwand gerecht werden, zu betreiben oder zu fördern, ihre Verfügbarkeit und Nutzung zu fördern und sich bei der Entwicklung von Normen und Richtlinien für universelles Design einzusetzen " (Art. 4 Abs. 1 lit. f UN-BRK).

Zur Gestaltung von Produkten, Gebäuden und öffentlichem Raum ist die Infrastruktur hinzuzunehmen, die Susan Leigh Star $(1999,377)$ ethnographisch analysiert hat und sie als relational und ökologisch charakterisiert. Infrastruktur prägt und beeinflusst menschliche Interaktionen als nicht zu unterschätzender Faktor: im öffentlichen Raum, an Arbeitsorten und -strukturen sowie im Privatleben. Die Gesellschaft gewinnt dadurch, Infrastruktur als bedeutsamen sozialen Faktor zu erachten, wie Latour $(1991,129)$ zum Verhältnis von Technik und Sozialem hervorhebt: „If we abandon the divide between material infrastructure on the one hand and social superstructure on the other, a much larger dose of relativism is possible“.

\subsection{Umweltorientierte Erfassung von Barrieren}

Während juristisch, medizinisch und gesundheitswissenschaftlich Barrieren individuumsorientiert konstruiert sind und abgeleitete Studien Barrieren meist in Bezug zum einzelnen Menschen und dessen Fähigkeiten und Beeinträchtigungen erfassen (vgl. Schröder u.a. 2017), fokussieren andere Studien auf die physische Umwelt (vgl. Stark u.a. 2007) bzw. konkretisiert auf städtische Umwelten (vgl. Clarke u.a. 2011) oder bauliche Umwelten (vgl. Gamache u.a. 2017). Die Studien gründen sich entweder auf das Soziale Modell (vgl. Stark u.a. 2007, 130), auf die ICF (vgl. Clarke u.a. 2011) oder auf die UN-BRK, die Disability Studies und die ICF (vgl. Gamache u.a. 2017; für eine Analyse der unterschiedlichen Ausrichtungen des Behinderungsbegriffs in ICF und UN-BRK vgl. Hirschberg 2018). In den Studien, die hier exemplarisch herangezogen werden, stehen Barrieren als Teil der physischen, baulichen oder städtischen Umwelt im Zentrum, weil Umweltbedingungen als entscheidend für Partizipation in der Community aller Menschen, auch behinderter Menschen, erachtet werden.

Aufgrund der eigenständigen Komponente der Umweltfaktoren lässt sich die ICF auch zur Beurteilung von gesellschaftlichen Benachteiligungen behinderter Menschen einsetzen, indem Barrieren nicht nur bezogen auf den einzelnen Menschen, sondern auch als strukturelle Hindernisse beurteilt werden. Hiergegen könnte eingewendet werden, dass die Umweltfaktoren (wie alle Komponenten der ICF) so konzipiert sind, dass sie auf ein Individuum ausgerichtet sind und nicht zur Überprüfung gegebener Barrieren für größere Gruppen dienen. Wenn die Umweltfaktoren jedoch am Konzept des Universal Design oder den Kriterien für Barrierefreiheit ausgerichtet werden, kann überprüft werden, wie die Umwelt barrierefrei zu gestalten ist. 
Da Universal Design zur Nutzung für alle Menschen konzipiert ist, lässt sich der öffentliche Raum unabhängig von einem Individuum mit einer spezifischen Behinderung beurteilen. So gibt es Instrumente zur Bewertung der Umwelt wie den Fragebogen zur Erfassung der physischen Umwelt für mobilitätsbeeinträchtigte Menschen, der mit dem Ziel der Verbesserung des Lebensumfeldes und des Wohlbefindens von Menschen mit Mobilitätsbeeinträchtigungen entwickelt wurde (vgl. Stark u.a. 2007, 124). Die Community Health Environment Checklist (CHEC) wurde entwickelt und von mobilitätsbeeinträchtigten Menschen validiert, um Orte der physischen Umgebung objektiv zu messen (vgl. ebd., 125). Hierzu gehören 36 Destinationen (vgl. ebd., 123), wie z.B. „Performance venues, transportation facilities, outdoor leisure areas" (ebd., 127), die auf Gestaltungsmerkmale wie „accessible bathrooms, very lightweight doors, signs in the building “ überprüft wurden (ebd., 129). Der CHEC kann als Grundlage zur Entwicklung anderer Instrumente der partizipativen Erforschung von konkreten Umgebungen für andere Behinderungsgruppen dienen (vgl. ebd., 130).

Die Bedeutung städtischer Umweltfaktoren für die Partizipation erwachsener behinderter Menschen mit Seh-, Hör- oder Mobilitätsbeeinträchtigungen wurde analysiert, indem die Daten des Chicago Community Adult Health Study (von 2001 bis 2003) für eine Befragung von 1.225 Personen zugrunde gelegt wurden gemäß der drei Partizipationsindikatoren „interpersonal interaction, obtaining preventive health care und voting" (Clarke u.a. 2011, 1674). Hierfür wurden Charakteristika der städtischen Umgebung „quality of the streets, and sidewalks, volume of traffic, public transit line“" (ebd., 1676) erhoben, anhand derer die Teilhabe eingeschränkt oder erleichtert sein kann. Als Ergebnisse wurde erkannt, dass der Teilhabegrad je nach den Umwelteigenschaften variiert: „Poor street conditions, heavy traffic, and low residential security" behindern die Teilhabe der gewählten Partizipationsindikatoren (ebd., 1683). Es wurde resümiert, dass „simple changes in urban built environments may facilitate the full participation of all persons in society" (ebd.).

In einem weiteren Ansatz sollte zur Implementierung der UN-BRK in Quebec ein umfangreicher, anwendbarer Wortschatz entwickelt werden, um hiermit die räumliche Umwelt präzise beschreiben zu können (vgl. Gamache u.a. 2017, 236). Die entwickelte Taxonomie basiert auf dem Human Development Model - Disability Creation Process Modell (HDM-DCP), einem Review bestehender Nomenklaturen und wurde um photographische Feldanalysen ergänzt, anhand derer die Beachtung aller vorhandenen Elemente im öffentlichen Raum überprüft wurde (vgl. ebd., 237). Die Forschungsabteilung „Right to equality and inclusive Cities“ am von Henri Lefebvre 1968 gegründeten „Centre interdisciplinaire de recherche en réadaptiation et intégration sociale (CIRRIS)“ hat mit behinderten Menschen in der Feldanalyse kooperiert, um die relevanten Objekte und Infrastruktur unter deren Expertise zu identifizieren, zu beurteilen und ihre Modifizierung zu planen 
(vgl. ebd., 239f.). Bei der Entwicklung der Taxonomie wurde auch auf die ICF Bezug genommen und positiv hervorgehoben, dass diese ebenso wie der HDMDCP mit der UN-BRK kompatibel sind und sie durch die Disability-Studies-Perspektive mit der entwickelten Taxonomie vereinbar würden (vgl. ebd., 247). Auch die Sustainable Development Goals (SDGs) der Vereinten Nationen bildeten eine wichtige Orientierung für die Fokussierung auf Umweltfaktoren, um Behinderung als Aufgabe in die Teilhabe aller Menschen einzubeziehen.

Kurz zusammengefasst: Umweltfaktoren können auch unabhängig von einer Person zur Erfassung von Barrieren - in der Perspektive des Gleichstellungsrechts eingesetzt werden.

\section{Schlussfolgerungen: Reflexion umweltorientierter Erhebung von Barrieren}

Beeinträchtigungen können nicht verhindert werden, passende technische oder personelle Assistenz ermöglichen jedoch volle gesellschaftliche Teilhabe.

Meist werden Barrieren sozialwissenschaftlich als Hindernis für Einzelne begriffen, wie auch als Frage im repräsentativen Teilhabe-Survey (2017) nach individuell erlebten Barrieren deutlich wird (Schröder u.a. 2017; Schäfers \& Schachler in diesem Band). Hingegen wird über andere Professionen wie Architektur, Ingenieurswissenschaften und auch ökonomische oder bürgerrechtliche Interessen an der gesellschaftlichen Eingliederung behinderter Menschen vermittelt, Barrieren als Hindernisse des öffentlichen (und auch privaten) Raums und damit als Hindernisse der Gesellschaft zu begreifen. Der Verpflichtung, Barrieren erst gar nicht entstehen zu lassen bzw. sie abzubauen und hierbei auch die Prinzipien Universellen Designs umzusetzen, kommt der deutsche Staat bisher nur ungenügend über die „weitgehend wirkungsarmen Instrumente der Zielvereinbarung und Verbandsklage“" nach, die zudem „der Zivilgesellschaft die Verantwortung für die Durchsetzung von Fortschritten“ auferlegen (DIMR 2015, 18; vgl. Frankenstein 2018, 241).

Barrierefreiheit lässt sich besonders dann als Mittel zu gesellschaftlicher Partizipation verstehen, wenn der staatlichen Umsetzungsverpflichtung Rechnung getragen wird und Barrierefreiheit auch im Sinne von Universellem Design geschaffen wird. Wie durch die nordamerikanischen Studien verdeutlicht, lassen sich sowohl die ICF als WHO-Behinderungsklassifikation als auch die UN-BRK als Menschenrechtsinstrument nutzen, um an Gebäuden und dem öffentlichen Raum das Ausmaß von Barrieren für Menschen mit unterschiedlichen Beeinträchtigungen zu erheben.

Eine gesellschaftsorientierte Erhebung von Barrieren ist also möglich und bereits wissenschaftlich fundiert. Hiermit kann ohne eine Erhebung individueller Beeinträchtigungen die staatliche Verpflichtung umgesetzt werden, Barrieren zu 
erfassen und abzubauen, damit alle behinderten Menschen an der Gesellschaft vollständig und gleichberechtigt teilhaben und in Würde leben können (vgl. Hirschberg \& Valentin 2020).

Die Perspektive der Disability Studies bzw. auch von Behindertenrechtsbewegungen wird bisher weniger beachtet als die traditionell stark individuumsorientierte Perspektive medizinischer und Gesundheits-Professionen. Der Zugang zu gesellschaftlichen Einrichtungen dient jedoch auch der Ausübung individueller Freiheiten und deren Ermöglichung a priori durch barrierefreie Gestaltungen von Orten, Objekten und auch institutionellen Bedingungen und gesellschaftlichen Dienstleistungen ohne Vorurteile, also ohne einstellungsbedingte Barrieren. Wenn also Barrieren abgebaut werden, ist dies nicht nur für behinderte Gesellschaftsmitglieder, sondern auch für alle anderen als potentiell zukünftig selbst behinderte Menschen nützlich. Dementsprechend ist zu empfehlen, in eine barrierefreie Gestaltung des öffentlichen (und auch privatwirtschaftlichen) Raums flächendeckend zu investieren, um den Zugang für alle zu ermöglichen.

\section{Literatur}

Bourdieu, P. (1987): Die feinen Unterschiede - Kritik der gesellschaftlichen Urteilskraft. Frankfurt/M.: Suhrkamp.

Campbell, F. (2009): Contours of Ableism. The Production of Disability and Abledness. London: palgrave.

Charlton, J. (2006): The Dimensions of Disability Oppression: An Overview. In: L. Davis (Ed.): The Disability Studies Reader. New York: Taylor \& Francis, 217-227.

Clarke, P., Ailshire, J., Nieuwenhuijsen, E. \& Kleijn de Vrankrijker, M. (2011): Participation among adults with disability: The role of the urban environment. In: Social Science \& Medicine 72, 1674-1684.

Degener, T. (2003): Behinderung als rechtliche Konstruktion. In: P. Lutz, T. Macho, G. Staupe \& H. Zirden (Hrsg.): Der [im-]perfekte Mensch. Metamorphosen von Normalität und Abweichung. Köln: Böhlau, 448-466.

DIMR - Deutsches Institut für Menschenrechte, Monitoringstelle zur UN-BRK (2015): Parallelbericht an den UN-Fachausschuss für die Rechte von Menschen mit Behinderungen. Berlin: DIMR.

Fisseler, B. (2015): Universal Design im Kontext von Inklusion und Teilhabe - Internationale Eindrücke und Perspektiven. In: Recht \& Praxis der Rehabilitation (2), 45-51.

Frankenstein, A. (2018): Universelles Design und Zugänglichkeit der Arbeitsplätze. In: G. Wansing, F. Welti \& M. Schäfers (Hrsg.): Das Recht auf Arbeit für Menschen mit Behinderungen. Internationale Perspektiven. Baden-Baden: Nomos, 227-245.

Gamache, S., Grenier, Y., Fougeyrollas, P., Edwards, G. \& Mostafavi, M.A. (2017): Developing a taxonomy of the built environment for disability studies. Methodological insights. In: Journal of Accessibility and Design for All 7 (2), 236-266.

Gugutzer, R. (2012): Verkörperungen des Sozialen. Neophänomenologische Grundlagen und soziologische Analysen. Bielefeld: transcript.

Hall, S. (1980): Cultural Studies: two paradigms. In: Media, Culture and Society 2(1), 57-72.

Hirschberg, M. (2018): Konzeptualisierungen von Behinderung in der ICF und der UN-BRK und deren Beitrag zur Verwirklichung des Rechts auf Arbeit. In: G. Wansing, F. Welti \& M. Schäfers (Hrsg.): Das Recht auf Arbeit für Menschen mit Behinderungen. Internationale Perspektiven. Baden-Baden: Nomos, 109-130. 
Hirschberg, M. (2014): Ethische Richtlinien für Forschung und Wissenschaft - Menschenrechtsbasierte Grundlagen gemäß Artikel 31 der UN-Behindertenrechtskonvention. In: E. Mührel \& B. Birgmeier (Hrsg.): Perspektiven sozialpädagogischer Forschung. Methodologien - Arbeitsfeldbezüge - Forschungspraxen. Wiesbaden: VS, 347-380.

Hirschberg, M. (2012): Barrieren differenzierter beurteilen - Vorschläge zur Weiterentwicklung der Behinderungs-Klassifikation der Weltgesundheitsorganisation. In: Teilhabe 51, 20-24.

Hirschberg, M. (2009): Behinderung im internationalen Diskurs. Frankfurt/M.: Campus.

Hirschberg, M. \& Valentin, G. (2020): Verletzbarkeit als menschliches Charakteristikum. In: D. Brehme, P. Fuchs, S. Köbsell \& C. Wesselmann (Hrsg.): Disability Studies im deutschsprachigen Raum. Zwischen Emanzipation und Vereinnahmung. Weinheim: Beltz Juventa, 89-95.

Hurst, R. (2003): The International Disability Rights Movement and the ICF. In: Disability and Rehabilitation 25 (11-12), 572-576.

Köbsell, S. (2012): Wegweiser Behindertenbewegung. Neues (Selbst-)Verständnis von Behinderung. Neu-Ulm: AG SPAK.

Kronauer, M. (2010): Exklusion. Die Gefährdung des Sozialen im hoch entwickelten Kapitalismus. Frankfurt/M.: Campus.

Latour, B. (1991): Technology is society made durable. In: J. Law (Ed.): A Sociology of Monsters. Essays on Power, Technology and Domination, Sociological Review Monograph 38. London: Routledge, 103-131.

Leach Scully, J. (2004): What is a disease? Disease, Disability and their definitions. In: European Molecular Biology Organization reports (7), 650-653.

Leigh Star, S. (1999): The Ethnography of Infrastructure. In: American Behavioural Scientist, 43 (3), 377-391.

Netzwerk Artikel 3(2018): Schattenübersetzung. Übereinkommen über die Rechte von Menschen mit Behinderungen. Online unter: http://www.nw3.de/attachments/article/130/BRK-Schattenuebersetzung-3-Auflage-2018.pdf. (Abrufdatum: 17.03.2020).

Oliver, M. (1992): Changing the Social Relations of Research Production? In: Disability, Handicap \& Society 7 (2), 101-114.

Paul, C. (2010): Disability Rights \& Universal Design. Social Welfare History Project. Online unter: https://socialwelfare.library.vcu.edu/issues/disability/disability-rights-universal-design/(Abrufdatum: 20.03.2020).

Schröder, H., Steinwede, J., Schäfers, M., Kersting, A. \& Harand, J. (2017): Repräsentativbefragung zur Teilhabe von Menschen mit Behinderungen - Zwischenbericht. BMAS-Forschungsbericht 492. Online unter: https://www.bmas.de/SharedDocs/Downloads/DE/PDF-Publikationen/Forschungsberichte/fb-492-repraesentativbefragung-behinderung.pdf. (Abrufdatum: 20.03.2020).

Siegrist, J. (2002): Reducing social inequalities in health: Work-related strategies. In: Scandinavian Journal of Public Health 30, 49-53.

Stark, S., Hollingsworth, H., Morgan, K. \& Gray, D. (2007): Development of a measure of receptivity of the physical environment. In: Disability and Rehabilitation 29 (2), 123-137.

Story, M. (2001): Principles of Universal Design. In: W. Preiser \& E. Ostroff (Eds.): Universal Design Handbook. New York: McGraw-Hill.

Union of the Physically Impaired Against Segregation (UPIAS) (1975): Fundamental Principles of Disability. London: UPIAS.

Wansing, G. \& Westphal, M. (Hrsg.) (2018): Migration, Flucht und Behinderung. Herausforderungen für Politik, Bildung und psychosoziale Dienste. Wiesbaden: Springer VS.

Wansing, G. \& Westphal, M. (Hrsg.) (2014): Behinderung und Migration. Inklusion, Diversität. Intersektionalität. Wiesbaden: VS.

Welti, F., Frankenstein, A. \& Hlava, D. (2019): Angemessene Vorkehrungen und Sozialrecht. In: Die Sozialgerichtsbarkeit (6), 317-325.

Wengler, A. (2012): Ungleiche Gesundheit. Zur Situation türkischer Migranten in Deutschland. Frankfurt/M.: Campus. 
Wiedemann, S. \& Roßberg, K. (2016): Barrierefreiheit für psychisch kranke Menschen, was ist das? In: Psychosoziale Umschau (3), 4-5.

World Health Organization \& World Bank (2011): The World Report on Disability. New York: WHO.

World Health Organization (2001/2005): The International Classification of Functioning, Disability and Health/Die Internationale Klassifikation von Funktionsfähigkeit, Behinderung und Gesundheit. Genf/Neu-Isenburg: WHO.

Zola, I. (1993): Self, Identity and the Naming Question: Reflections on the Language of Disability. In: Social Science and Medicine 36(2), 167-173. 


\section{Oliver Sträter}

\section{Universal Design - Gestaltung der Zugänglichkeit von Arbeitssystemen für Menschen mit Behinderung}

\section{Einleitung}

Menschen mit Behinderung haben ein Recht auf angemessene Gestaltung der Arbeitssysteme, sodass sie trotz ihrer Behinderung am Arbeitsleben möglichst vollständig teilhaben können. Oft wird diese Sichtweise als defizitäre Sichtweise einer bestimmten Personengruppe (der behinderten Personen) interpretiert.

Die Sichtweise der ergonomischen Arbeitsgestaltung ist jedoch eine andere. Arbeitsmittel sind insgesamt so zu gestalten, dass der Mensch hinsichtlich seiner Eigenschaften und Fähigkeiten völlig unabhängig davon, welche Einschränkungen er hat, optimal unterstützt wird. Dies gilt auch für Einschränkungen jeglicher Art, die sich auf die Gestaltung der Arbeit auswirken können. Im klassischen Bereich der Arbeitsgestaltung sind dies beispielsweise und insbesondere Körpergröße, Hör- oder Seh-Fähigkeiten. Tische müssen höhenverstellbar gestaltet, auditive oder visuelle Informationsübermittlung für alle Altersgruppen wahrnehmbar sein.

Insofern stellen die Anforderungen für die Arbeitsgestaltung für Menschen mit Behinderung keine neue methodische Fragestellung in der ergonomischen Gestaltung dar. Behinderungen sind dabei im Sinne der Arbeitsgestaltung bestimmte funktionale Einschränkungen für bestimmte Tätigkeiten und keine Einschränkung per se. Ein einfaches Beispiel: In der Automobilindustrie spielt die Haptik in der Produktgestaltung von Oberflächen eine große Rolle. Ausschlaggebend für eine Tätigkeit zur Beurteilung der Haptik ist, inwieweit dieser Informationskanal ausgebildet und sensibilisiert ist, die haptische Qualität von Oberflächen zu bewerten. Andere Informationskanäle oder gar Bewegungseinschränkungen der Person, die diese Tätigkeit durchführt, sind irrelevant, mitunter ist vielleicht sogar eine Person, die sich allein auf diesen Sinneskanal konzentrieren kann, qualitativ besser in dieser Tätigkeit.

Die neue Anforderung hinsichtlich der Arbeitsgestaltung beinhaltet deshalb aus ergonomischer Sicht im Wesentlichen folgende Aspekte:

- die Heterogenität unterschiedlicher funktionaler Einschränkungen, die in der Arbeitsgestaltung zu berücksichtigen sind,

- die Passung von Tätigkeit und funktionalen Eigenschaften der Person und

- eine wirksame Planung und Implementierung von Gestaltungslösungen. 
Inwieweit eine ergonomische Arbeitsgestaltung hinsichtlich dieser drei Aspekte gelingen kann, wird in den folgenden Abschnitten diskutiert.

\section{Umgang mit Heterogenität}

\subsection{Homogenität versus Flexibilität}

Prinzipiell ergeben sich zwei unterschiedliche Herangehensweisen zum Umgang mit Heterogenität. Die erste Möglichkeit ist, dass eine Gestaltungslösung gewählt wird, die allen Personen gleichermaßen gerecht wird (Homogenität). Die zweite Möglichkeit ist, individuelle Gestaltungslösungen zu entwickeln, die dann idealerweise auch noch modifiziert werden können und flexibel sind (Flexibilität).

Der erste Ansatz sieht auf den ersten Blick wie der einfachere und der zweite als der aufwändigere und damit auch kostenmäßig ungünstigere aus. Dies muss aber nicht notwendigerweise so sein. Ein universelles Design kann beispielsweise im Bereich der Produktgestaltung günstig sein, jedoch ungünstig in der Implementierung, da das Design so universell ausgelegt sein kann, dass nur eine geringe Nutzergruppe das System gut bedienen kann. Der überwiegende Teil der Nutzer empfindet das System aber unzureichend und nutzt es gar nicht erst.

Individuelles Design erfordert jedoch eine flexible und adaptive Gestaltungslösung. Die entsprechenden funktionalen Einschränkungen müssen deshalb bekannt sein und auch wirksam durch das Design berücksichtigt werden. Eine 100 \%ige Abdeckung aller funktionalen Einschränkungen und vollständiger Flexibilität und Attraktivität wird dabei auf der Gestaltungsseite einen hohen Aufwand bedeuten.

Nimmt man diesen als, trade-off bezeichneten Sachverhalt, dass sowohl universelles Design als auch vollständige Individualität bezüglich der Gesamtkosten ungünstige Extreme sind, bietet sich für eine realistische Umsetzung ein Kompromiss zwischen beiden Gestaltungsprinzipien an. Ein System muss also möglichst universell und möglichst individuell gestaltet sein.

Gelingen kann dies nie in vollständiger Art und Weise, und man kann nie erwarten, dass ein Produkt zu $100 \%$ von allen Personen genutzt werden kann. Das gilt im klassischen Bereich der Arbeitsgestaltung genauso wie im Bereich der Gestaltung für behinderte Personen. Insgesamt ist eine optimale Gestaltung eine Frage der Auslegung. Auslegung bedeutet die Festlegung einer Systemgestaltung auf der Basis der größtmöglichen Wirkung in seinem Anwendungsspektrum.

Ein einfaches Beispiel zum Thema Auslegung findet sich wiederum beim Automobil: Die Sitzverstellung wird anhand von Personendaten festgelegt. Kleine Personen müssen die Möglichkeit haben, den Sitz nach vorne zu schieben, und große Personen müssen ihn nach hinten schieben können. Andererseits hat ein Automobil gewisse Abmaße, die die Sitzverstellung limitieren. Die Frage der Auslegung der 
Sitzverstellung ist deshalb, mit welcher Limitierung die günstigste Gestaltung erreicht wird.

\subsection{Erfordernis für Flexibilität}

Schlussendlich müssen auch Beschäftigungsverhältnisse und Vertragsrechte zwischen den Interessenvertretungen dahingehend optimiert werden. Allzu oft behindern rigide Denkweisen hinsichtlich der Gleichbehandlung von Mitarbeitenden den Einsatz von Menschen mit Behinderung im normalen Arbeitsleben. Hier ist eine Flexibilisierung erforderlich, die dann für die Personaleinsatzplanungen auch die Anwendung der hier genannten Prinzipien der Passung von Tätigkeit und funktionalen Eigenschaften gewährleistet.

Insgesamt würde so nicht nur eine Sicherung der Beschäftigung von Menschen mit Behinderung gelingen, sondern eine pluralistische und damit flexible Mitarbeiterschaft, die dann in der Lage ist, eine Organisation auch besser durch dynamische Veränderungen zu führen.

\subsection{Erfordernis für Homogenität}

Die Anforderung an Homogenität ergibt sich allein daraus, dass im Bereich der Produktfertigung nicht beliebig viele Variationen eines Produktes umgesetzt werden können. Aus diesen Anforderungen ist das Konzept ,One-Size-Fits-All erwachsen: Statt Flexibilisierung des Produkts vorzusehen, wird das Produkt so gestaltet, dass es allen Personen gleichermaßen gerecht wird.

Ein solches Produkt geht jedoch zulasten der Attraktivität und damit der Akzeptanz einer Nutzergruppe. Warum sollte beispielsweise Benutzergruppe A wegen bestimmter Eigenschaften von Benutzergruppe B ein aus ihrer Sicht unattraktives und gegebenenfalls sogar nutzerunfreundliches Produkt verwenden?

Psychologisch bedeutet dies, dass hierdurch automatisch eine Abwertung der Benutzergruppe B durch Benutzergruppe A geschieht. Dies gilt auch für Menschen mit Behinderung. Wenn beispielsweise eine Gestaltungslösung aufgrund von Anforderungen der Menschen mit Behinderung aus Sicht eines nicht-behinderten Menschen als gebrauchsuntauglich erachtet wird, beschwert sich diese Nutzergruppe. Das wiederum hat Auswirkungen auf die Wahrnehmung der behinderten Menschen als Personengruppe.

Allerdings haben sich die Produktionsvorgänge in den letzten Jahren erheblich dadurch verändert, dass auch Produktionsprozesse flexibel gestaltet werden können, beispielsweise durch dreidimensionale Drucker oder Digitalisierung im Herstellungsprozess.

Aufgrund der insgesamt unterschiedlichen Vor- und Nachteile der Homogenisierung und Flexibilisierung ist deshalb eine Optimierung erforderlich. Diese beruht im ersten Schritt auf der optimalen Passung von Tätigkeit und funktionalen Eigenschaften. 


\section{Passung von Tätigkeit und funktionalen Eigenschaften}

\subsection{Analyse funktionaler Einschränkungen}

Eine wichtige analytische Tätigkeit zur Optimierung einer Gestaltungsmaßnahme ist die Analyse der Passung von Tätigkeit und funktionalen Eigenschaften einer Person. Dieses Vorgehen ist im Rahmen der Arbeitswissenschaft bzw. Ergonomie insbesondere im Bereich der Softwareergonomie eine typische Herangehensweise. Zunächst werden die im Arbeitssystem erforderlichen Tätigkeiten analysiert, systematisiert und bezogen auf ihr Anforderungsprofil klassifiziert. Im weiteren Schritt werden die erforderlichen Eigenschaften der Personen diesem Anforderungsprofil zugeordnet. Die Passung von Anforderungen und Eigenschaften der Personen werden dann hinsichtlich passend bzw. unpassend für die Tätigkeit bewertet.

Dieses prinzipielle Vorgehen kann natürlich in analoger Weise auf Menschen mit Behinderung angewendet werden. Hierdurch entsteht eine Bewertungsmatrix, mit welcher die Passung eines Menschen für eine bestimmte Tätigkeit einhergeht. Diese Passung kann dann wiederum in zweierlei Hinsicht ausgewertet werden:

- Es kann eine Zuordnung der Personen zu den Tätigkeiten durchgeführt werden, in denen die Einschränkungen der Personen keinen Einfluss auf die Durchführbarkeit der Tätigkeit haben. Beispiel: Bei überwiegend sitzenden Tätigkeiten spielt die Tatsache, ob jemand in einem Rollstuhl sitzt oder nicht, keine Rolle.

- Es kann eine Gestaltungsmaßnahme hinsichtlich der Tätigkeiten eruiert werden, um trotz der Einschränkungen eine Durchführbarkeit der Tätigkeit zu gewährleisten. Beispiel: Bei Einschränkung des visuellen Systems wird für das Lesen von Texten ein auditives Unterstützungssystem bereitgestellt.

\subsection{Bewertungs-Matrix}

Das prinzipielle Vorgehen und Resultat einer solchen Analyse zeigt Tabelle 1. Auf der Vertikalen sind die Tätigkeitsanforderungen klassifiziert und auf der Horizontalen der Tabelle die funktionalen Einschränkungen. In der Analyse werden Kombinationen von Tätigkeitsanforderungen und Einschränkungen in diesem Beispiel mit drei Wertungskategorien belegt: unkritisch, neutral, kritisch.

Alternativen können zusätzlich den Aspekt der Kompensierbarkeit abbilden, indem zwischen ,unkritisch', ,kritisch aber kompensierbar" und ,kritisch und nicht kompensierbar' unterschieden wird. Kompensation kann dann z.B. durch neue Technologien oder organisatorische Umplanungen stattfinden.

Innerhalb der Matrix können dazu die Gemeinsamkeiten über Spalten, Zeilen oder Bereiche hinweg analysiert werden.

Eine Betrachtung über die Spalten erlaubt die Identifikation von Tätigkeitsbereichen, die trotz einer funktionalen Einschränkung ohne Weiteres durchführbar sind. Eine Betrachtung über die Zeilen erlaubt die Identifikation von Personalprofilen, die erfüllt sein müssen, oder entsprechendem Assistenzbedarf, der dann mit 
Unterstützung durch Assistenzsysteme von einer Person durchführbar wird (siehe dazu Abschnitt 5). Eine Betrachtung über Bereiche erlaubt die Identifikation organisatorischer Maßnahmen, wie unterschiedliche Personen mit unterschiedlichen Einschränkungen gemeinsam und ggf. mit Assistenzsystemen im Arbeitsleben zu integrieren sind.

Bezüglich organisatorischer Maßnahmen ergeben sich beispielsweise Tandemmodelle im Personaleinsatz (einfaches Beispiel: Eine blinde und eine gehörlose Person ergänzen sich hinsichtlich ihrer Fähigkeiten der Informationsaufnahme). Bezüglich neuer Technologien wird das Anwendungspotenzial dieser Methodik in Abschnitt 5 getrennt beschrieben.

Prinzipielle Maßnahmen können sein:

- Unterstützung einer funktionalen Einschränkung

- Tandem-Modell zur gegenseitigen Ergänzung von funktionalen Einschränkungen

- Tätigkeiten in der Arbeitsgestaltung so einteilen oder auslegen, dass Behinderungen in der jeweiligen Tätigkeit nicht zu Einschränkungen führen

Tab. 1: Analyse der Passung von Tätigkeit und funktionalen Eigenschaften

\begin{tabular}{|c|c|c|c|c|c|}
\hline & & Funktion & nale Einschränkur & ungen & \\
\hline 2 & 1 & 2 & 4 & 5 & 10 \\
\hline 3 & 1 & 3 & 4 & 6 & 8 \\
\hline 2 & 3 & 6 & 6 & 5 & 7 \\
\hline 1 & 2 & 4 & 5 & 5 & 4 \\
\hline 2 & 3 & 2 & 6 & 4 & 4 \\
\hline 1 & 6 & 1 & 6 & 10 & 5 \\
\hline 3 & 4 & 9 & 6 & 9 & 6 \\
\hline 3 & 1 & 8 & 5 & 7 & 10 \\
\hline 2 & 1 & 2 & 4 & 9 & 9 \\
\hline 1 & 3 & 2 & 6 & 10 & 7 \\
\hline 2 & 3 & 1 & 4 & 7 & 8 \\
\hline 2 & 1 & 3 & 6 & 2 & 9 \\
\hline 2 & 2 & 3 & 6 & 3 & 10 \\
\hline
\end{tabular}

\subsection{Gestaltungs-Auslegung}

In der anthropometrischen Gestaltung ist das Verfahren der Zuordnung von Tätigkeiten zu physischen Eigenschaften des Menschen ein übliches und etabliertes Verfahren. Hierzu werden Verteilungen eines bestimmten Auslegungsparameters (zum Beispiel Körpergröße) angenommen bzw. empirisch bestimmt und über Maximierungsfunktionen die bestmögliche Auslegung des Systems bestimmt. Die 
Logik für dieses Vorgehen ist die sogenannte Perzentilierung. Diese besagt, dass die Verteilung an bestimmten Punkten abgeschnitten und damit das Auslegungskriterium bestimmt wird. Abbildung 1 zeigt das prinzipielle Vorgehen.

Über die Bildung von Populationen wird die Effektivität einer Gestaltungsmaßnahme dahingehend abgeschätzt, wie weit sie maximal der Population entspricht. Hierdurch löst der Ansatz der Perzentilierung die oben diskutierten Probleme der Heterogenität, indem das Optimum zwischen Flexibilität und Homogenität methodisch bzw. empirisch bestimmt wird. Ein solches Verfahren ist leider für die informatorische Arbeitsgestaltung wenig etabliert und sollte im Sinne des Umgangs mit funktionalen Einschränkungen jedoch auch für informatorische Arbeiten bzw. Personengruppen mit funktionalen Einschränkungen etabliert werden.

\section{Planung und Implementierung von Gestaltungslösungen}

\subsection{Bedeutung der Planungsphase für Gestaltungslösungen}

Ein wesentliches Problem bei der erfolgreichen Umsetzung von Gestaltungslösungen ist eine rechtzeitige und gute Planung. Insgesamt muss man davon ausgehen, dass nach $20 \%$ des Planungszeitraums ca. $80 \%$ der Ressourcen bereits fest vergeben sind. Das heißt planerische Aspekte, die später als in dem ersten Fünftel eines Projektes Berücksichtigung finden sollen, werden mit nur $20 \%$ der Projektressourcen umsetzbar sein.

Durch diesen einfachen Sachverhalt ergibt sich, dass Planungsaspekte, die nicht von vornherein in der Projektkonzeption berücksichtigt sind, de facto in den späten Projektphasen keine Beachtung mehr finden. Allzu oft werden deshalb zur Lösung der eingangs aufgeführten Heterogenitätsproblematik homogene Produkte oder Prozesse erstellt, teilweise sogar wider besseres Wissen, was deren Anwendungsmöglichkeiten angeht. Der Grund liegt schlichtweg darin, dass keine finanziellen Ressourcen für Flexibilität im Rahmen des Projektes oder der Entwicklungsarbeit zur Verfügung stehen.

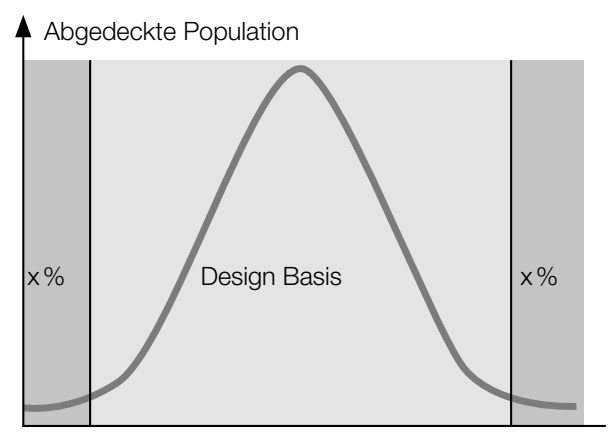

Abb. 1: Perzentilierung als methodisches Vorgehen zur Bestimmung von Gestaltungskriterien 
Um eine ausreichende Flexibilität hinsichtlich der Umsetzung von Gestaltungslösungen für Personen mit funktionalen Einschränkungen zu gewährleisten, ist somit eine planerische Berücksichtigung der Aspekte in der Anfangskonzeption eines Produktes oder einer anderen Gestaltungslösung erforderlich.

\subsection{Planungsunterstützung}

Zur Planungsunterstützung wurde ein Konzept entwickelt, welches bedeutende Anforderungsgrößen für ein neues (oder bestehendes) Planungsumfeld analysiert und frühen Planungsphasen zur Verfügung stellt. Das Verfahren wird als Screening-Verfahren bezeichnet. Ein entsprechendes Tool zur Planung und Unterstützung erlaubt die Darstellung zusammenhängender gebündelter Information im Rahmen einer Moderation (Sträter u.a. 2020).

Bei sehr komplexen Projektplanungen kann diese Moderation in einer multimedialen Umgebung stattfinden. Hierdurch können Wechselwirkungen und Problemfelder besser erkannt werden. Die Bewertung kann zusätzlich durch die Einbettung von thematisch verknüpften Inhalten angereichert werden, wie beispielsweise Diagrammen, Videosequenzen, Prozessbeschreibungen oder Mitschriften aus vergangenen Besprechungen (Krämer u.a. 2008). Hierdurch gelingt eine Reduktion des kognitiven Aufwandes, und die Verarbeitung von Informationen kann durch die multimediale Aufbereitung des Informationsmaterials reduziert werden (Craik \& Lockhart 1973). Das Screening-Verfahren ist als Software-Tool realisiert und kann in klassischen Seminarräumen, besser aber in multimedial unterstützten Räumen, eingesetzt werden.

Es liefert eine integrierte Vorgehensweise, bei der Überlegungen zur Planungszuverlässigkeit in Projekten und Prozessen während des Lebenszyklus angestellt werden. Es wird von einem Moderator geleitet; am Verfahren selbst sind alle entscheidungsrelevanten Experten für einen Planungsprozess gleichermaßen beteiligt. Dies sind üblicherweise Produktplaner, Kaufleute, Designer, Arbeitssicherheit, Gesundheitsmanagement, Betriebsräte oder weitere Akteure im Betriebs- oder Produktmanagement. Im Rahmen einer Planung für behinderte Menschen kämen dann noch Schwerbehindertenvertretungen und gegebenenfalls Benutzergruppen mit spezifischen Behinderungen hinzu.

Die unterschiedlichen Perspektiven werden in einer Screening-Session und mithilfe des Software-Tools von einem Moderator integriert und Planungsinformationen gesammelt. Dabei erleichtert das Software-Tool die Moderation des Workshops und führt den Anwender systematisch durch alle betroffenen Aspekte. Ein Projektmanager erhält so Einblicke in bestehende mögliche Wechselwirkungen und einen gebündelten Überblick über die zu prüfenden Anforderungen. Das Screening gibt außerdem Hinweise auf einen Ablaufplan und ein mögliches Zeitkontingent, welches einzuhalten ist. 
Durch den Prozess

- stehen so Planungsinformationen für eine proaktive Systemgestaltung zur Verfügung,

- erlangen die unterschiedlichen Akteure gegenseitiges Problembewusstsein,

- werden Konflikte zwischen den Akteuren reduziert, die sich ansonsten negativ auf die Produkt- oder Prozessgestaltung auswirken würden,

- erhält eine Organisation eine robustere Planungsgrundlage für ein Produkt oder einen Prozess.

Abbildung 2 zeigt den Vergleich zwischen der klassischen und proaktiven Systemgestaltung mittels Screening-Ansatz.
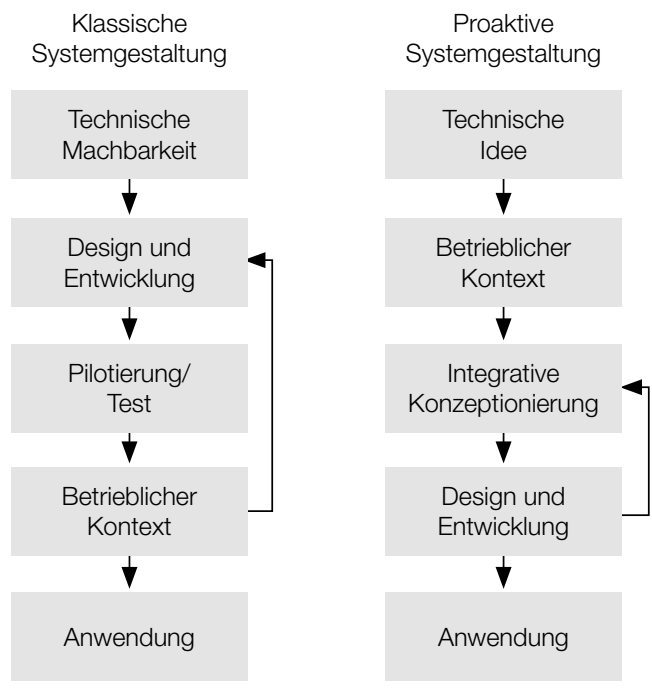

Abb. 2: Vergleich klassischer versus proaktiver Systemgestaltung mit Hilfe eines Screening-Ansatzes (in Anlehnung an Sträter u.a. 2012)

Bei der Durchführung eines Safety-Screenings in einer Planungsphase findet eine systematische Bewertung der systemabhängigen Anforderungsgrößen im Wirkungskreislauf statt. Dies bildet die Grundlage zur Berücksichtigung möglicher Zielkonflikte innerhalb der verschiedenen Anforderungsgrößen bei der strategischen Planung. Das Safety-Screening-Verfahren basiert auf grundlegenden Design-Kriterien aus der Gestaltung sicherheitsrelevanter Branchen wie Luftfahrtindustrie, Kernenergie, petrochemische Industrie, See- und Eisenbahnindustrie. 
Das Screening-Tool umfasst derzeit vier Hauptkategorien, die zur Unterstützung des Planungstools dienen. In Abbildung 3 wird der Aufbau dargestellt. Neben dem betrieblichen Management und dem Arbeitssystem sind ebenfalls die Anwendungsebene und die Organisationsebene mit in den Planungsprozess zu integrieren. Das Verfahren kann auf den Bereich der Behinderung ohne Weiteres erweitert werden, indem spezifische Haupt- und Unterkategorien zu den Belangen behinderter Menschen gebildet werden.

\begin{tabular}{|c|c|c|c|}
\hline \multicolumn{4}{|l|}{ Hauptfragen } \\
\hline $\begin{array}{l}\text { Betriebliches } \\
\text { Management }\end{array}$ & Arbeitssystem & $\begin{array}{l}\text { Anwendungs- } \\
\text { ebene }\end{array}$ & $\begin{array}{l}\text { Organisations- } \\
\text { ebene }\end{array}$ \\
\hline \multicolumn{4}{|l|}{ Unterfragen } \\
\hline Definierte Abläufe & $\begin{array}{l}\text { Tätigkeitsgestaltung \& } \\
\text { Handlungsspielraum }\end{array}$ & $\begin{array}{c}\text { Lernen \& } \\
\text { Verbesserung }\end{array}$ & $\begin{array}{c}\text { Leistungsvorgaben } \\
\text { \& Kontrolle }\end{array}$ \\
\hline Produktionsprozess & Ergonomie & Zusammenarbeit & Mitbestimmung \\
\hline $\begin{array}{l}\text { Qualifizierung/ } \\
\text { Ausbildung }\end{array}$ & $\begin{array}{c}\text { (Digitale) } \\
\text { Werkerassistenz }\end{array}$ & $\begin{array}{l}\text { Kommunikation \& } \\
\text { Informationsflüsse }\end{array}$ & Führung \\
\hline Verantwortlichkeiten & $\begin{array}{l}\text { Technische Ausstat- } \\
\text { tung/Hilfsmittel }\end{array}$ & Partizipation & Datenschutz \\
\hline
\end{tabular}

Abb. 3: Hauptkategorien des Screening-Tools

Das Screening-Tool stellt zu jeder Kategorie Moderationsfragen zur Verfügung, die mit Hilfe eines Moderators vom Entwicklerteam beantwortet und bewertet werden müssen. Durch eine Dokumentation der Diskussion in freien Textfeldern zu den Fragen entsteht wichtige Planungsinformation, die dann für die weitere Entwicklung genutzt werden kann. Daneben kann eine Bewertung der Bedeutung der Aspekte durch die Teilnehmer dahingehend geschehen, welche Bedeutung der Aspekt hat und inwieweit er besonderer Aufmerksamkeit bedarf (Sträter 2019b).

Abbildung 4 zeigt eine exemplarische Darstellung der quantitativen Ergebnisse. Liegt die Punktzahl für einen bestimmten Aspekt außerhalb des schattierten Bereichs, muss diesem Thema in den späteren Phasen besondere Aufmerksamkeit gewidmet werden. Die Ergebnisse liefern Planungsinformationen für weitere Schritte in der Einführung/Etablierung von Gestaltungslösungen. 


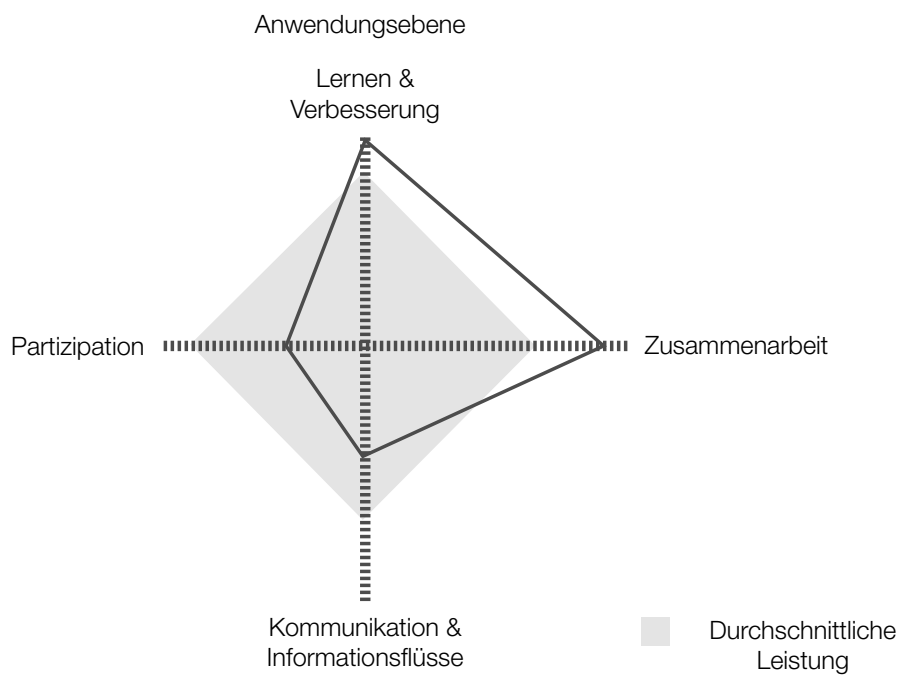

Abb. 4: Ergebnisse des Screening-Tools (beispielhafter Auszug)

Das Screening-Verfahren unterstützt in einem Änderungsprozess zusammenfassend folgende wesentliche Ziele:

- Identifikation von Anforderungen in einem frühen Stadium der Entwicklung von Konzepten, einschließlich positiver und negativer Auswirkungen geplanter Änderungen auf die Belastungen der Mitarbeitenden oder Nutzenden.

- Priorisierung von Problembereichen und Terminierung von weiteren Maßnahmen und Bewertungen.

- Integration von ergonomischen mit finanziellen und betrieblichen Entscheidungskriterien einer Prozess- oder Produktplanung.

- Koordination der Anforderungen einer Betreiberorganisation mit den zuständigen Verantwortlichen.

Durch die Vorgehensweise des Screening-Verfahrens werden die Beteiligten in frühen wie späteren Projektphasen über unklare Anforderungen besser informiert. Darüber hinaus fördert das Safety-Screening ein gemeinsames Verständnis und eine gemeinsame Sicht auf die wesentlichen Anforderungen der Praxis. Verzögerungen eines Projektes aufgrund von Planungsdefiziten oder unklaren Verantwortlichkeiten werden dadurch vermieden.

\subsection{Iterative Projektbegleitung}

Im Rahmen des Projektverlaufes müssen Festlegungen zur Flexibilisierung und Homogenisierung entsprechend weiterverfolgt werden. Hierzu bietet es sich an, im Rahmen der ohnehin stattfindenden Entwicklungsmeetings das Screening- 
Tool regelmäßig einzusetzen. Hiermit wird das Screening-Verfahren ein integraler Bestandteil einer Projektabwicklung und erlaubt die rechtzeitige und nachhaltige Integration von ergonomischen Anforderungen.

Auch können rechtzeitig unterschiedliche begleitende Maßnahmen zur Gestaltungsmaßnahme festgestellt und initiiert werden. Treten beispielsweise in Screening-Prozessen Erkenntnisse hinsichtlich einer Flexibilisierung der Personaleinsatzplanung und organisatorischen Arbeitsgestaltung zutage, können frühzeitig Personalqualifikationen und Weiterbildungsmaßnahmen definiert werden. Werden Produktanforderungen identifiziert, können entsprechende Prozesse zur Auslegung des Produktes rechtzeitig initiiert werden.

\section{Assistenz als Ansatz zur Auflösung der Heterogenität}

\subsection{Assistenz funktionaler Einschränkungen}

Die oben diskutierte Problematik der Heterogenität kann - neben einer Optimierung - den Trade-Off zwischen Flexibilisierung und Homogenisierung auch durch ergänzende technische Hilfsmittel aufösen. Hierzu könnte die im Abschnitt 3.2 vorgeschlagene Optimierungsmatrix dahingehend erweitert werden, durch welche technischen Hilfsmittel kritische Kombinationen kompensiert werden können. Welche Assistenz-Systeme sinnvoll sind, kann durch die in Abschnitt 4 vorgestellte Verfahrensweise des Screenings identifiziert werden.

Im Bereich der Arbeitsgestaltung halten derzeit unterschiedlichste technische Hilfsmittel Einzug. Sie reichen von informatorischer Unterstützung, wie beispielsweise Datenbrillen, bis hin zu Exo-Skeletten. Alle diese Hilfsmittel dienen der Kompensation funktioneller Einschränkungen und werden deshalb als Assistenzsysteme bezeichnet - dies nicht allein im Bereich von Behinderung, sondern auch in klassischen Bereichen der Arbeitsgestaltung wie der Montage und Fertigung bei Tätigkeiten, die für den Menschen mit seinen normalen Eigenschaften ansonsten nicht durchführbar wären (vgl. Burkhardt \& Sträter 2017). Typisches Beispiel ist ein Exo-Skelett zur motorischen Unterstützung beim Heben von sehr schweren Lasten, wobei das Muskelskelettsystem des Menschen stark geschädigt werden würde. Auch Datenbrillen mit Darbietung virtueller Informationen und weitere digitale Assistenzsysteme werden bereits heute als eine Möglichkeit gesehen, um den Arbeitsalltag von Beschäftigten zu erleichtern. Langfristig werden diese Technologien immer weiter ihren Weg in die digitalisierte Arbeitswelt finden (BAUA 2018).

\subsection{Möglichkeiten der Assistenz durch Zustandserkennung}

Inwieweit unterschiedliche geistige oder körperliche Einschränkungen über ein Assistenzsystem unterstützt werden können, hängt zunächst einmal davon ab, inwieweit das Assistenzsystem in der Lage ist, einen unterstützungswürdigen $\mathrm{Zu}$ - 
stand des Nutzers zu erkennen. Ein Assistenzsystem muss also anhand von Frühindikatoren erkennen, ob entsprechende Assistenzfunktionen ausgelöst werden sollen. Assistenzsysteme können so eine Person hinsichtlich aller Aspekte menschlicher Tätigkeiten - wie Wahrnehmung, Kognition und Motorik - frühzeitig mit Kompensationshandlungen unterstützen.

Hierzu ist zunächst ein entsprechendes System zur Erfassung des Nutzerzustandes erforderlich. Um sowohl die informatorischen, kognitiven sowie die motorischen Zustände einer Person zu erfassen, wurde das System CeyeBERMANS entwickelt (Klippert 2018; Arenius u.a. 2016; Sträter u.a. 2018). Der cEYEberman ist ein kombiniertes Mess-System zur Messung psychischer und physischer Belastungen in einem beliebigen Tätigkeitsumfeld einer Person. Es beinhaltet Sensorik, Blickbewegungs-Kameras und Inertial-Sensoren, die in einem leichten Anzug integriert sind (Abbildung 5).

Das Blickverhalten und die Bewegungsabläufe werden während der Interaktion mit der Arbeitsumgebung oder der Bildschirminteraktion in Echtzeit aufgezeichnet und analysiert. Die speziell entwickelte Analysesoftware untersucht die er-

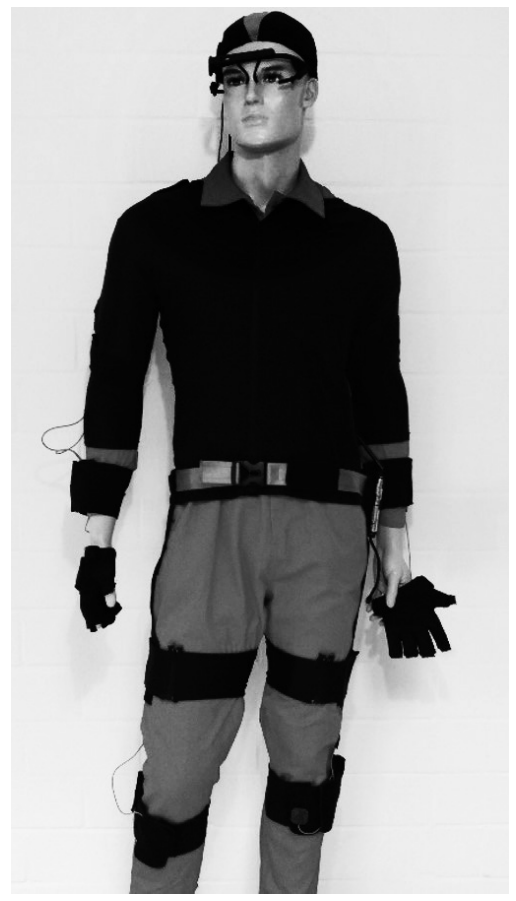

Abb. 5: CeyeBERMANS Mess-System für physische und psychische Belastungen gonomischen Bedingungen bei der Interaktion zwischen Mensch und Maschine. CeyeBERMANS hilft, die Sicherheit und Zuverlässigkeit von Aktivitäten und der gesamten Arbeitsumgebung zu optimieren. Die solide Sensortechnologie ermöglicht den Einsatz selbst unter ungünstigen Umgebungsbedingungen (wie Staub, Wasserdampf usw.). Das System ist in dieser Kombination weltweit einmalig und praxiserprobt in vielfältigen echten Arbeitsumgebungen vom Flugbereich zur Messung von Pilotenbelastungen, über Prozesskontrolle bis hin zur häuslichen Pflege und selbst bei Tätigkeiten in sehr ungünstigsten Arbeitsbedingungen (Hochdruckreinigung).

Das Mess-System ist modular aufgebaut und kann mit weiteren Sensoren ergänzt werden. Mit einem EEG (Elektroenzephalogramm) können beispielsweise Zustände wie Aufmerksamkeit, Wachheit und Reaktionsbereitschaft klassifiziert werden. Weitere Module dienen der Erfassung auditiver Signale (durch Mikrofone) oder 
haptischer Signale bzw. Kräfte durch Druckmessmatten. Abbildung 6 zeigt die Erweiterung des Systems mit dem EEG.

Mit dem CeyeBERMANS-System steht ein Verfahren zur Erkennung von psychischen Zuständen zur Verfügung. Um aus den Messwerten assistenzbedürftige Zustände abzuleiten, müssen diese noch klassifiziert werden. Hierzu werden die dynamischen Muster in den gemessenen menschlichen Verhaltensdaten und psychophysiologischen Daten analysiert und von einem Klassifikationsansatz der künstlichen Intelligenz in Echtzeit klassifiziert.
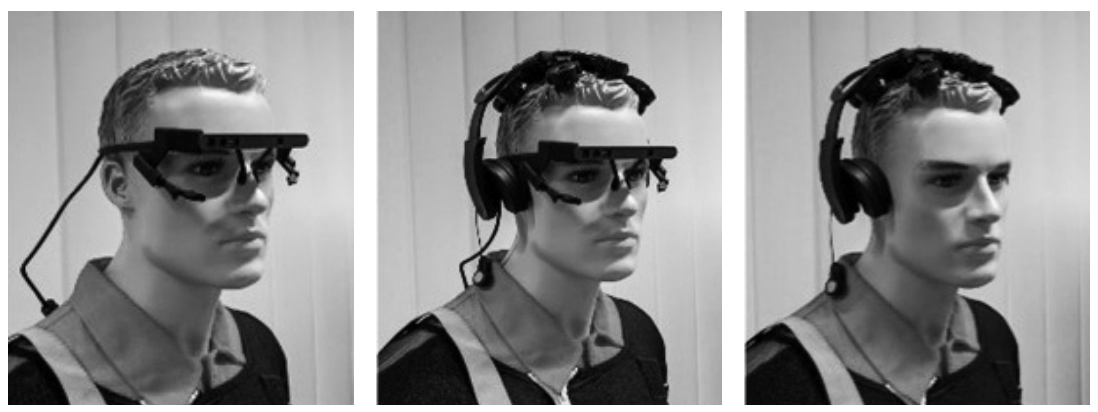

Abb. 6: Blickbewegungs-Messsystem (links), EEG (rechts) sowie deren Kombination (Mitte)

Ferner können Assistenzfunktionen oder Verhaltenshinweise über visuelles (Datenbrille), akustisches oder haptisches Feedback an die Person oder eine andere Person übermittelt werden (Burkhardt 2019).

Zur Erkennung von menschlichem Verhalten oder psychischen Zustandsveränderungen werden maschinelle Lernalgorithmen angewendet, die aufbauend aufTrainingsdaten statistische Modelle berechnen, anhand derer es möglich ist, aus einer Datenbasis neues Wissen zu generieren, indem Muster und Gesetzmäßigkeiten in den Daten erkannt und zur Beurteilung auf neue unbekannte Verhaltensweisen herangezogen werden. So kann zunächst durch die Quantifizierung der Blickbewegung die informatorische/mentale Belastung untersucht werden, basierend auf den Veränderungen der visuellen Aufmerksamkeit. Zudem können beispielsweise anhand der Pupillenweite die Tagesschläfrigkeit und damit der allgemeine Grad der Aktivierung erfasst werden. Aus dynamischen Veränderungsmustern im Blickverhalten können darauf aufbauend höherwertige kognitive Funktionen abgeleitet werden, wie die Identifizierung von Fehlerpotential während der Arbeit oder Denkmuster (Jennerich u.a. 2016; Jennerich 2018).

Blickbewegungserfassung kann auch zur Entwicklung einer proaktiven Tätigkeitsunterstützung genutzt werden, indem das Blickverhalten zur Erkennung von Tätigkeiten ausgewertet wird. Dies wiederum kann für ein kontextbezogenes Assistenzsystem verwendet werden. Der Prozess von der Datenaufbereitung bis hin 
zur Entwicklung eines statistischen Modells oder eines neuronalen Netzes gliedert sich dabei in mehrere Schritte, welche in Abbildung 7 dargestellt sind.

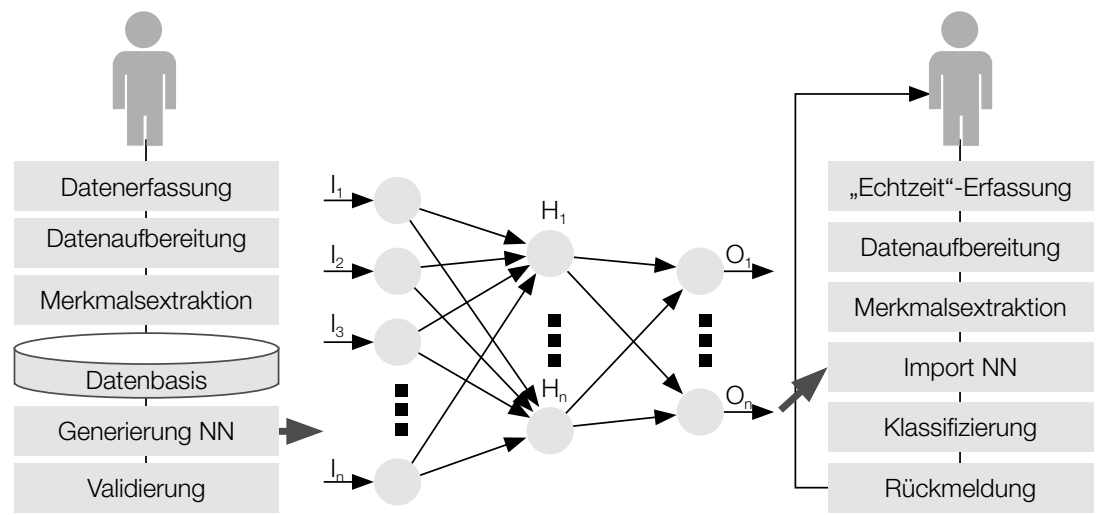

Abb. 7: Verfahren zur echtzeitfähigen Vorhersage und Assistenz anhand erfasster spezifischer Merkmale (nach Jennerich 2018)

Bevor die erhobenen Daten weiterverarbeitet werden können, müssen diese bereinigt und aufbereitet werden, damit keine ungewollten Artefakte mit in ein statistisches Modell einfließen. Um die Blickbewegungsdaten für das maschinelle Lernen zu verwenden, werden zunächst charakteristische Merkmale aus den Rohdaten extrahiert. Aufbauend auf diesen Merkmalen können Support-Vector-Machines oder Künstliche-Neuronale-Netze trainiert werden. Diese mathematischen Verfahren ermöglichen eine computergestützte Mustererkennung innerhalb der Datenströme. Die dynamischen Veränderungen werden durch psychologische oder physiologische Veränderungen provoziert und können entsprechend zugeordnet werden. Durch die Zuordnung der Veränderung von beliebigen Verhaltensdaten auf die Muster in den Datenströmen lernen die mathematischen Verfahren und können anschließend auf neue Verhaltensdaten angewendet werden, um diese zu klassifizieren. Auf Basis der Klassifikation können situative Veränderungen erkannt und entsprechende Maßnahmen zur Unterstützung einer Person initiiert werden. Das gesamte Verfahren ist auf beliebige Messdaten, beliebiges Verhalten und beliebige Klassifikationen anwendbar und kann so problemlos auf Zustände der geistigen Behinderung angewandt werden.

\subsection{Möglichkeiten der Assistenz zur Kompensation funktionaler Einschränkungen}

Sind psychische Zustände hinreichend erfasst und kategorisiert, können entsprechende Assistenzfunktionen ausgelöst werden. Die Anwendungsbereiche sind dabei vielfältig und die Technologien weiterentwickelt flexibel einsetzbar. 
Beispielsweise können Inhalte, die auditiv vorliegen, für Gehörlose durch ein Übertragungssystem auf eine Datenbrille projiziert werden. Personen mit visuellen Einschränkungen können mittels Raumerkennung und Vibrationssensoren Rückmeldungen über den vor ihnen liegenden Bewegungsraum übermittelt bekommen; auch wäre eine automatisierte Erkennung von Schrift oder Blindenschrift mit entsprechender auditiver Rückmeldung durch solch ein System realisierbar. Personen, die unter motorisch bemerkbaren Anfällen wie Zittern oder einem Tremor leiden, können durch Frühindikatoren rechtzeitig gewarnt und ihre Handlungskontrolle verbessert werden.

Hierzu können unterschiedliche Systeme eingesetzt werden. Sogenannte AR (Augmented Reality) Datenbrillen können zur Visualisierung von 3D-Objekten im „realen“ Raum eingesetzt werden und eine entsprechende visuelle Assistenz bieten (Azuma 1995). Diese kann zur Aufmerksamkeitssteuerung genutzt werden, um beispielsweise die Orientierungsleistung hinsichtlich kritischer Informationen zu verbessern oder um die im Fokus liegenden optischen Informationen durch visuelle Vergrößerung oder auditive Unterstützung entsprechend aufzubereiten.

Andere Rückmeldesysteme könnten im Bereich der Körperwahrnehmung dazu dienen, die Handlungssteuerung zu verbessern und so Fehlhaltungen zu korrigieren oder fehlerhafte Eingriffe in ein System rechtzeitig zu erkennen und somit Handlungsfehler zu vermeiden. Solche Systeme werden derzeit nicht nur im Bereich der Unterstützung von Menschen mit Behinderung genutzt, sondern auch zur Unterstützung von Personen beispielsweise in Montage und Fertigung (Burkhardt u.a. 2016). Abbildung 8 zeigt ein solches System.

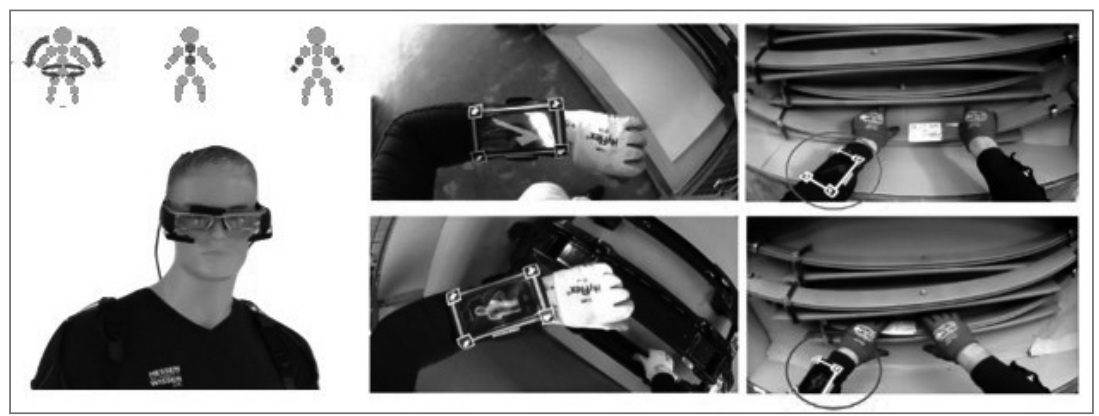

Abb. 8: Visuelle und taktile Rückmeldesysteme zur Assistenz von Personen; links visuelle Rückmeldung mit einer AR-Datenbrille; rechts haptische Rückmeldung mithilfe eines Mobiltelefons (nach Burkhardt 2019)

Auf der linken Seite sind Projektionen in einer AR-Datenbrille aufgezeigt, die der Person ihre Körperhaltung zurückmeldet. Auf der rechten Seite ist über ein Mobilte- 
lefon eine haptische Rückmeldung über Bewegungsverhalten und mögliche Fehler dargestellt.

Solche Systeme können nicht nur zur Assistenz in Echtzeit, sondern auch zur Aus- und Weiterbildung hinsichtlich der Fähigkeiten einer Person genutzt werden, beispielsweise im Bereich des Trainings für blinde oder gehörlose Menschen.

\section{Ausblick}

Für die moderne Arbeitsgestaltung ist eine kombinierte Vorgehensweise erforderlich. Unabhängig davon, ob es um Arbeitsgestaltung für behinderte Menschen oder für eine bestimmte Benutzergruppe geht, ist ein Kompromiss zwischen Homogenität der Gestaltungslösung und Flexibilität der Gestaltungslösung erforderlich, um eine optimale Arbeitsgestaltung zu gewährleisten (VDE 2011).

In diesem Beitrag wird beschrieben, wie das Optimum für dieses Problem gefunden werden kann. Ergonomische Arbeitsgestaltung bedeutet dabei nicht spezifische Arbeitsgestaltung für Menschen mit Behinderung, sondern optimale Arbeitsgestaltung in der Kombination aller Mitarbeitenden, ob sie eine Behinderung haben oder nicht (Klippert u.a. 2013).

Durch das hier vorgeschlagene methodische Vorgehen gelingt deshalb zunächst eine inhaltliche Inklusion von Menschen mit Behinderung dadurch, dass die Arbeitsgestaltung hinsichtlich aller Bedürfnisse optimiert wird und eine systemische Perspektive eingenommen wird (Sträter 2019b).

Dadurch, dass Anforderungen von Menschen mit Behinderung nicht als gesonderte Gestaltungsmaßnahme in dieser Methode aufgeführt sind, gelingt auch eine bessere Inklusion dieser Personengruppen über einen homogenen methodischen Ansatz, der keine zusätzlichen Aufwände erfordert und damit das Thema gegebenenfalls für Praktikerinnen und Praktiker schwieriger macht.

\section{Literatur}

Arenius, M., Klippert, J., Athanassiou, G. \& Sträter, O. (2016): Analysis of Dynamic Performance Data for the Assessment of Cognitive States: Results from Aviation, Assembly Tasks and Maritime Transportation. In B. Deml, P. Stock, R. Bruder, \& C.M. Schlick (Hrsg.): Advances in Ergonomic Design of Systems, Products and Processes. Berlin und Heidelberg: Springer, 209-222.

Azuma, R. (1995): A Survey of Augmented Reality. Online unter: https://www.cs. unc.edu/ -azuma/ ARpresence.pdf. (Abrufdatum: 21.06.2020).

BAUA (2018): Expertenworkshop Datenbrillen - Aktueller Stand von Forschung und Umsetzung sowie zukünftiger Entwicklungsrichtungen vom 07.03.2018. BAUA. Dortmund. Online unter: https://www.baua.de/DE/Angebote/Veranstaltungen/Dokumentationen/Neue-Technologien/Datenbrillen-2018.html. (Abrufdatum: 21.06.2020).

Burghardt T., Di Valentin C., Sträter, O., Krummich, J. \& Raabe, D. (2016): PREFLOW-Preventive Workflows: Intelligentes Assistenzsystem zur präventiven Steuerung von Arbeitsprozessen. In GfA (Hrsg.), Arbeit in komplexen Systemen. Digital, vernetzt human?! (B.1.6). Dortmund: GfA-Press. 
Burghardt, T. \& Sträter, O. (2017): PREFLOW - Preventive Workflows: Intelligentes Assistenzsystem zur präventiven Steuerung von Arbeitsprozessen. Schlussbericht. Universitäts- und Technische Informationsbibliothek (TIBKAT). Hannover.

Burghardt, T. (2019): Ergonomieassistenz - Entwicklung und Evaluierung von Assistenzkonzepten in der visuellen, auditiven und taktilen Modalität zur Verbesserung ergonomischen Verhaltens. Universität Kassel, Kassel.

Craik, F.I.M., \& Lockhart, R.S. (1972): Levels of processing: A framework for memory research. Journal of Verbal Learning and Verbal behaviour, 11, 671-684.

Jennerich, M. (2018): Proaktive Tätigkeitsunterstützung: Adaptive Tätigkeitserkennung in Abhängigkeit der Pupillendynamik und Entwicklung einer situativen Assistenz (Dissertation). Universität Kassel, Kassel.

Jennerich, M., Meyer, K., Sträter, O. (2016): Entwicklung und Evaluation eines proaktiven Assistenzsystems zur Unterstützung von Personalhandlungen zur Erhöhung der Prozesssicherheit. In: Gesellschaft für Arbeitswissenschaft (Hrsg.), VerANTWORTung für die Arbeit der Zukunft. Dortmund.

Klippert, J. (2018): Augmented Ergonomics. Theorie und Praxis der systemischen Arbeitsanalyse. Baden-Baden: Nomos Verlagsgesellschaft.

Klippert, J., Ott, A. \& Sträter, O. (2013): Bericht über die Arbeiten der Universität Kassel - A+O - im Rahmen des AAL@Home-Projektes. Universität Kassel, Arbeits- und Organisationspsychologie. Kassel.

Krämer, N.C. (Hrsg.) (2008): Medienpsychologie. Schlüsselbegriffe und Konzepte. Stuttgart: Kohlhammer.

Sträter, O. (2019a): Wandel der Arbeitsgestaltung durch Digitalisierung Transfer von Erkenntnissen aus der Sicherheitsforschung auf die Arbeitsgestaltung in der digitalen Transformation. Zeitschrift für Arbeitswissenschaft. 73:252-260. Springer.

Sträter, O. (Hrsg.) (2019b): Risikofaktor Mensch? - Zuverlässiges Handeln gestalten. Beuth Verlag.

Sträter, O., Korteweg, H., Nollet, J., Everdij, M., Athanassiou, G., Arenius, M. \& Kraan, B. (2012): Safety scanning - An approach to manage safety in the Single European Sky. Paper the PSAM/ ESREL Conference 2012. Helsinki.

Sträter, O., Schmidt, S., Saki, M. \& Hölker, J. (2020): Nutzen eines Scanning-Verfahrens zur Unterstützung der Planung digitalisierter Systeme. Beitrag Z.1.14. GfA, Dortmund (Hrsg.): Frühjahrskongress 2020, Berlin.

Sträter, O., Stephanie, S., Stache, S., Saki, M., Wakula, J., Bruder, R. \& Ditchen, D. (2018): ULinien-Montagesysteme - Instrumente zur Gefährdungsbeurteilung und arbeitswissenschaftliche Gestaltungsempfehlungen zur Prävention: Abschlussbericht. BG Holz \& Metall. Düsseldorf.

VDE (2011). Qualitätskriterien im Umfeld von AAL - Produkte - Dienstleistungen - Systeme 6/2011“. BMBF/VDE Innovationspartnerschaft AAL (Hrsg.). VDE Verlag GmbH, Berlin, Offenbach, 2011. (ISBN 978-3-8007-3397-2). 


\title{
Empirische Erfassung
}

\author{
Friedrich Dieckmann
}

\section{Verständnis und empirische Erfassung von Barrieren aus ökologisch-psychologischer Sicht}

Barrieren erschweren oder behindern die Teilhabe an gesellschaftlich üblichem Alltagsgeschehen und an sozialen Beziehungen. Barrieren zu identifizieren und aufzuheben, das Wissen bereitzustellen, um inklusive Settings zu schaffen, das sind wichtige Ziele der Teilhabeforschung. Allerdings tut sich die Teilhabeforschung schwer damit, die vielfältigen Arten von Barrieren im Alltagsleben für Menschen mit unterschiedlichen Beeinträchtigungen theoretisch zu begreifen, Befunde zu systematisieren und letztendlich Empfehlungen für die Gestaltung funktionierender inklusiver Settings zu geben.

An dieser Stelle könnte die Teilhabeforschung von dem ökologisch-psychologischen Rahmenkonzept des Behavior Settings (BS) profitieren. Aus Sicht der ökologischen Psychologie lässt sich die Ökologie eines Menschen durch die sozial-kulturellen Geschehenssysteme und die sozialen Netzwerke beschreiben, an denen er/sie jeweils teilhat. Roger Barker (1968) hat auf der Basis umfangreicher Beobachtungsstudien - gerade auch von Kindern und Jugendlichen mit Behinderung im öffentlichen Geschehen - das Konzept des Behavior Settings in den 1960-70er Jahren entwickelt, um Geschehenssysteme auszumachen und ihr Funktionieren zu analysieren. Darauf aufbauend lassen sich hinderliche sowie förderliche Bedingungen für die Teilnahme von Menschen mit Beeinträchtigungen an Behavior Settings formulieren.

Ziel meines Beitrags ist es, das Potenzial des Behavior Setting-Konzepts für die Erforschung von Barrieren und von förderlichen Bedingungen in der Teilhabeforschung deutlich zu machen. Dabei stütze ich mich auf Gerhard Kaminski (1995), der Barkers Behavior Setting-Konzept psychologisch weiterentwickelt und bereits auf Menschen mit Behinderungen angewandt hat. 


\section{Anforderung der Teilhabeforschung an eine psychologische Analyse}

Wird Behinderung gemäß des ICF-Modells (vgl. DIMDI \& WHO 2005) als Störung der Teilhabe begriffen, richtet sich der Blick auf die außerhalb der beeinträchtigten Person liegenden Umweltbedingungen, die die Teilhabe erschweren bzw. verhindern (Barrieren) oder die sie erleichtern bzw. überhaupt ermöglichen (Förderfaktoren). Das, was im Alltagsgeschehen zu einer Barriere oder einem Förderfaktor wird, lässt sich nur durch die Analyse des wechselseitigen Zusammenspiels einer Person und ihrer Umwelt im Handeln bestimmen. Für die Identifikation von Barrieren und von förderlichen Bedingungen sind wir auf psychologische Forschung angewiesen, die in der Lage ist, Mensch-Umwelt-Transaktionen theoretisch und forschungsmethodisch abzubilden. Gleichzeitig soll das individuelle Handeln mit seinen Verhaltens- und Erlebenskomponenten Zielpunkt der Analyse bleiben.

Wo finden sich in der Psychologie Ansätze, die das leisten können? Überwiegend werden in psychologischen Ansätzen - wie in der Kognitionspsychologie, im Behaviorismus, in der Psychoanalyse oder in der Neuropsychologie - nur wenige Aspekte der Umwelt eines Menschen und die oft sehr reduziert theoretisch-begrifflich und forschungsmethodisch artikuliert. Es interessiert mehr, wie die Umwelt aus der Innensicht der Personen subjektiv wahrgenommen bzw. kogniziert wird und welche emotional-motivationalen, kognitiven und verhaltensbezogenen Reaktionen isolierte Umweltaspekte auslösen. Auch in Kurt Lewins berühmter Formel $\mathrm{V}=\mathrm{f}(\mathrm{P}, \mathrm{U})-$ Verhalten ist eine Funktion von Person und Umwelt - ist die Umwelt als subjektiv repräsentierter Lebensraum konzipiert, der seine Kraft entfaltet. Die „Umweltvergessenheit“ der Psychologie und die mangelnde ökologische Validität psychologischer Erkenntnisse sind ab den 1970er Jahren auch im deutschsprachigen Raum breit diskutiert worden (vgl. Kaminski 1976). Es stellte sich die Frage, wie die Ökologie des Menschen aus psychologischer Sicht beschrieben und konzipiert werden kann.

\section{Psychologische Ökologie und Teilhabe}

Der bereits erwähnte Kurt Lewin begann in seiner angewandten Forschung in den USA, die Ökologie menschlichen Handels stärker in den Blick zu nehmen. In einer Feldstudie, die Einflussfaktoren für die Veränderung von Essgewohnheiten ermitteln sollte, kam er zu dem Schluss, dass das Nahrungsangebot in den Läden, auf den Märkten und in den Gärten sowie die komplexe Wahlentscheidung der Hausfrau als Gatekeeper für den Haushalt wichtigere Faktoren seien als die Essensvorlieben der übrigen Familienmitglieder (vgl. Lewin 1943). Die Verände- 
rung des Angebots und sozialer Normen für die Hausfrau wurden als Schlüsselelemente für Veränderungen identifiziert.

Die ökologische Wende in der Psychologie ist aber vor allem mit dem Namen Roger Barker (1903-1980) verbunden. Barker war einige Jahre Mitarbeiter bei Kurt Lewin gewesen (vgl. Kaminski 2002, 194). Er beobachtete das Verhalten von Kindern im Tagesablauf und registrierte, dass sie sich im Alltag fast durchweg jeweils „kontextgerecht“ verhielten, obwohl die Kinder doch sehr unterschiedliche Temperamente und Persönlichkeiten hatten (Barker \& Wright 1955). Die Beobachtungsprotokolle zeigten, dass unterschiedliche Kinder sich in gleichen situativen Kontexten (z.B. Schulunterricht, gemeinsames Spielen) überraschend ähnlich verhielten. Für diese, das aktuelle Handeln bestimmenden, überindividuellen Umweltausschnitte prägte Barker den Begriff Behavior Setting (vgl. dazu im Detail Abschnitt 3 dieses Beitrags). Barker meinte, dass menschliches Alltagsleben primär in Form von Behavior Settings organisiert sei (vgl. Barker \& Associates 1978; Kaminski 2018). Er gründete mit seinen Mitarbeitenden in dem kleinen Ort Oskaloosa (mit damals 700 Einwohner*innen) in Kansas eine Feldstation mit dem Ziel, sämtliche öffentlichen Behavior Settings dieser Gemeinde über ein Jahr lang zu erfassen (vgl. Kaminski 2002, 194f). Mithilfe eines differenzierten Beobachtungsbogens, dem Behavior Setting-Survey, wurden Behavior Settings erfasst und beschrieben. Er berechnete auch einen Environment Richness Index (ERI), mit dem er die Lebensqualität eines Ortes kennzeichnete. Auf der Basis seiner Feldbeobachtungen entwickelte Barker (1968) ein systemtheoretisch orientiertes Rahmenmodell, in dem die strukturellen Komponenten und die interne Dynamik von Behavior Settings beschrieben werden. Barker litt seit seiner Jugend an einer Osteomyelitis, einer chronischen Knochen- und Knochenmarksentzündung, und war dadurch stark in seinen Bewegungsmöglichkeiten beeinträchtigt (vgl. ebd., 198). In „Qualities of Community Life“ (Barker \& Schoggen 1973) wird u.a. die Teilnahme von Kindern und Jugendlichen mit und ohne Behinderung an Behavior Settings in Oskaloosa (Kansas, USA) und Leyburn, einer kleinen Gemeinde in North Yorkshire (UK), in den Jahren 1954/55 und 1963/4 miteinander verglichen.

In seiner ökologischen Entwicklungstheorie stützt sich auch Uri Bronfenbrenner (1917-2005) bei der Formulierung seines Mikrosystems auf Barkers Behavior Setting-Konzept. Bronfenbrenner (1981) bettet das im Vergleich zu Barker unschärfer gefasste Mikrosystem in größere Umweltkontexte ein (Meso-, Exo- und Makrosysteme) und beschreibt Sozialisation als Entwicklung eines Individuums innerhalb eines Mikrosystems und im Übergang zwischen Mikrosystemen. Gerhard Kaminski hat die Nahtstelle zwischen dem Individualsystem und dem überindividuellen Behavior Setting psychologisch ausgearbeitet und auch den Zusammenhang zwischen der Teilnahme an Behavior Settings und sozialen Beziehungsgeschehen herausgestellt (vgl. Kaminski 1995, 55f.). Als Kooperationspartner im Zentrum für 
die interdisziplinäre Erforschung von Lebenswelten behinderter Menschen an der Universität Tübingen hat er zwei bemerkenswerte Artikel zu Behinderung aus ökologisch-psychologischer Perspektive verfasst, auf die in diesem Beitrag immer wieder Bezug genommen wird (Kaminski 1985; 1995).

Die individuelle Teilhabe an gesellschaftlichen Gütern (vgl. Day \& Dieckmann 1995) wird in der ökologischen Psychologie kontextualisiert. Erstens wird sie als Teilnahme an sozial-kulturell geprägten Geschehenssystemen, den Behavior Settings, betrachtet. Zweitens bedeutet Teilhabe die Einbindung einer Person in soziale Beziehungen und Beziehungsgefüge (soziale Netzwerke). Soziales Interaktions- und Beziehungsgeschehen findet innerhalb, aber auch außerhalb von Behavior Settings statt. Behavior Settings sind Orte des sozialen Aufeinandertreffens. In ihnen können sich soziale Interaktionen entfalten. Das Bild, das sich eine Person von anderen macht, wird auch durch den Teilnahmekontext bestimmt. Die Entstehung von persönlich gefärbten sozialen Interaktionen und Beziehungen hängt also eng mit der Begegnung in Behavior Settings zusammen.

\section{Das Behavior Setting-Konzept}

Ein Behavior Setting ist ein überindividuelles, konkretes sozial-kulturelles Geschehenssystem. Es ist der Kontext, in dem das Handeln von Individuen, Dyaden und Gruppen eingebettet ist. Stark angelehnt an Kaminski (1995, 51ff.) lassen sich die Merkmale eines Behavior Settings am Beispiel eines inklusiv angelegten Kunstateliers in einer Stadt in Baden-Württemberg ${ }^{1}$ veranschaulichen.

Das Kunstatelier hat klare raumzeitliche Grenzen. Zu dem Kunstatelier, das jeden Freitagvormittag für sechs Stunden bis in den Nachmittag hinein seine Türen öffnet, gehören drei angemietete Räume einer Grafikschule (Arbeitsraum, Rückzugsecke, Büronische). Sie befinden sich in einem alten Fabrikgebäude.

$\mathrm{Zu}$ der Teilnehmerschaft (Population des Behavior Settings) gehören u.a. Menschen mit Psychiatrieerfahrung, Grundschulkinder, Rentnerinnen und Rentner. Altersgemischt nehmen zwischen 10 und 15 Männer und Frauen mit und ohne künstlerische Vorbildung an einer Ateliersitzung teil.

Das Behavior Setting hat eine interne soziale Struktur. Es finden sich verschiedenartige funktionale Positionen, die abgestuft mit unterschiedlichen Rechten und Pflichten verbunden sind („Leader“, weitere herausgehobene Positionen, „einfache“ Teilnehmende, Zuschauer*innen). Beispielsweise gibt es eine Leiterin, eine Heilpädagogin, die die Räume öffnet, für die Materialien sorgt, die Werbung gestaltet, neue Teilnehmende einführt, das Geld verwaltet, hilft innerhalb des Kunstateliers kleine Workshops zu organisieren und außerhalb Ausstellungen zu

1 Das Beispiel ist abgewandelt der Masterthesis von Ann-Kathrin Götz (2018) über inklusiv angelegte Kunstateliers entnommen. 
veranstalten. Herausgehobene Positionen haben u.a. Teilnehmende inne, die einen Workshop leiten, oder jemand, der als studierter Kunsthistoriker Besucher*innen durch Ausstellungen des Künstlerateliers führt. Neben den Teilnehmenden, die im Atelier ihren künstlerischen Aktivitäten nachgehen, kommen auch immer wieder Besucher*innen, die einfach nur zuschauen wollen. Die Positionen sind also mit verschiedenen Rollenanforderungen verknüpft. Personen sind doppelt beansprucht, zum einen als Partizipanten an dem Behavior Setting, zum anderen als Individuen, die eigene Ziele verfolgen und Handlungsfreiräume innerhalb des Behavior Settings individuell ausgestalten.

Ein Behavior Setting zeichnet sich durch typische Handlungsmuster aus. In dem inklusiven Kunstatelier dominiert das künstlerische Gestalten: Malen, Zeichnen, Bildhauern. Das Atelier startet mit einem gemeinsamen Frühstück. Feste Gesprächsrunden zur Begrüßung oder Verabschiedung gibt es im Unterschied zu anderen derartigen Kunstateliers aber nicht. Ein Behavior Setting läuft nach einem bestimmten Programm ab, zu dem bestimmte phasenspezifische Abläufe, positionsspezifische Skripte und Regeln gehören. Das besagte Kunstatelier beginnt z.B. immer mit dem gemeinsamen Frühstück, bevor dann jede/r allein oder mit anderen künstlerischen Aktivitäten nachgeht. Immer wieder gibt es auch Pausengeschehen, in dem Tee und Kaffee getrunken und sich unterhalten wird. Ab und an findet im Rahmen des Kunstateliers auch ein Workshop statt, in dem eine künstlerische Technik bzw. ein Thema vorgestellt wird oder die Teilnehmenden eine Ausstellung konzipieren. Manchmal schließt sich an das Kunstatelier auch eine andere Aktivität an, wie z.B. ein gemeinsamer Museumsbesuch. Das Kunstatelier hat also eine gewisse Programmstruktur, bietet aber reichlich Freiräume für soziale Interaktionen, die nichts mit künstlerischen Aktivitäten zu tun haben müssen.

Das Kunstatelier lässt sich in verschiedene Subsettings unterteilen. So lädt der Rückzugsraum mit einem Sofa, Sitzgelegenheiten, Kaffeemaschine und Teekocher zum Ausruhen oder auch zu Gesprächen ein. Im Arbeitsraum finden sich Bereiche, in denen allein, zu zweit oder in kleinen Gruppen gemeinsam geschafft wird. $\mathrm{Zu}$ einem Behavior Setting gehören ein spezifisches räumlich-materielles Milieu und Verhaltensobjekte. Die unterschiedlichen Areale des Kunstateliers wurden bereits erwähnt. Im Arbeitsraum finden sich z.B. lange Arbeitstische, Staffeleien, Arbeitsstühle, Atelierfenster und Deckenleuchten, Materialien wie Leinwände, Papiere, Gouache, Acrylfarben oder Ytong-Steine. Das räumliche Milieu und die Verhaltensobjekte sind mehr oder weniger so arrangiert, dass sie das Handlungsgeschehen ermöglichen und erleichtern (Synomorphie zwischen Milieu und Handlungsgeschehen).

Behavior Settings haben eine spezifische Zugangscharakteristik. Das Behavior Setting übt auf seine unterschiedlichen Quellpopulationen einen unterschiedlichen Teilnahmedruck aus. Für einige ist die Teilnahme verpflichtend, für andere freiwillig, wiederum andere sind ausgeschlossen. Beispielsweise gehört es zu den Arbeits- 
pflichten der Leiterin, teilzunehmen und die ganze Zeit anwesend zu sein. Für die Psychiatrieerfahrenen ist die Teilnahme grundsätzlich zwar freiwillig, einige haben aber kein alternatives unterstütztes Tagesangebot. Für die meisten ist das Angebot aber gänzlich freiwillig. Grundsätzlich ausgeschlossen ist explizit keine Bevölkerungsgruppe. Da das Kunstatelier aber an einem Werktag tagsüber stattfindet, ist es de facto für viele Berufstätige oder auch Auszubildende nicht zugänglich.

Des Weiteren unterscheiden sich Behavior Settings in dem Grad der Autonomie. Die Leiterin und die Teilnehmenden haben zusammen z.B. eine große Freiheit in Bezug auf die Gestaltung des Ateliers. Allerdings ist die Leiterin für die Werbung in der Öffentlichkeit auf die Abstimmung mit den verschiedenen Kooperationspartnern angewiesen, die das Atelier finanzieren, was nicht immer leicht sei.

Drei Arten von Zielen beeinflussen das Geschehen: Programmziele (Funktion des Behavior Settings); individuelle Zielsetzungen, die über Programmziele hinausgehen, diese spezifizieren, modifizieren und erweitern; und Aufrechterhaltungsziele, die Störungen vermeiden bzw. beseitigen. Das Kunstatelier insgesamt hat zum Ziel, künstlerische Aktivitäten zu ermöglichen. Als Freizeitaktivitäten sollen sie in erster Linie Freude machen. Es besteht weder ein wirtschaftlicher Verwertungsdruck für die Produkte, noch müssen die Werke vorgegebenen ästhetischen Qualitätsansprüchen genügen. Die einzelnen Teilnehmenden verfolgen sehr unterschiedliche Ziele. Einige verstehen sich als Künstler*innen und wollen sich weiterentwickeln und weiterbilden. Für andere ist das Zusammensein mit anderen bei diesen Aktivitäten wichtiger. Viele schätzen das gesellige Beisammensein und die Bekanntschaften und Freundschaften, die im Rahmen dieses Settings auch gepflegt werden können. Andere sind einfach als Besucher*innen neugierig auf das, was geboten wird. Das Kunstatelier ist sehr offen für die verschiedensten individuellen Zielsetzungen. Gleichwohl wird jemand gebeten leise zu sein, wenn seine Lautstärke das künstlerische Tun beeinträchtigt. „Störenfriede“ werden ermahnt oder notfalls hinausgebeten. Die künstlerischen Aktivitäten und auch die Produkte werden geschützt. Der Rückzugsraum bietet zusätzlich Möglichkeiten der Regulation von Störungen innerhalb des Behavior Settings.

\section{Arten von Barrieren aus ökologisch-psychologischer Sicht}

Behavior Settings bilden neben sozialen Beziehungen die unmittelbar wirksamen Kontexte menschlichen Alltagshandelns. Welche Arten von Barrieren erschweren oder verhindern die Teilnahme an Behavior Settings? Unterscheiden lassen sich Barrieren der Zugänglichkeit von Behavior Settings, Barrieren für die Beteiligung am Behavior Setting-Geschehen und Barrieren, die die Veränderbarkeit von Behavior Settings betreffen. Ein Teil der Barrieren für eine Person mit Beeinträchtigung kann häufig durch die Unterstützung anderer Personen überwunden werden, worauf deshalb zusätzlich eingegangen wird. 


\subsection{Zugänglichkeit von Behavior Settings}

Behavior Settings müssen, erstens, vorhanden sein bzw. man muss sie kreieren, um an ihnen teilnehmen zu können (Vorhandensein eines Behavior Settings). Zweitens müssen sie für eine Person auffindbar sein, d.h. eine Person muss über ein bestimmtes Behavior Setting informiert sein bzw. sie muss sich leicht Informationen über das Behavior Setting beschaffen können (Auffindbarkeit eines Behavior Settings). Drittens muss ein Behavior Setting räumlich und zeitlich für eine Person tatsächlich erreichbar sein (raumzeitliche Erreichbarkeit eines Behavior Settings). Eine Person muss in der Lage sein, zu dem Zeitpunkt an den Ort zu kommen, an dem es stattfindet. Unüberwindbare Distanzen (z.B. aufgrund mangelnder Verkehrsmittel und Mobilitätshilfen), räumliche Zugangsbarrieren und unpassende Öffnungszeiten können die Teilnahme erschweren oder ganz verhindern.

Viertens kann die Zugangscharakteristik für Teilpopulationen sehr unterschiedlich sein:

- Zugangskriterien wirken sich selektiv hinderlich auf Teilpopulationen aus. So werden bestimmte Personengruppen von vornherein direkt (z.B. durch Altersgrenzen für bestimmte Kinofilme, Türsteher*innen vor Tanzclubs, Immatrikulation für Hochschulseminare) oder indirekt ausgeschlossen (z.B. durch hohe Teilnahmegebühren, Teilnahmebegrenzungen, Aufnahmeverfahren, die bestimmte Personen bevorzugen).

- Die Teilnahme an einem Behavior Setting kann für unterschiedliche Teilpopulationen mehr oder weniger verpflichtend sein (Verpflichtungsgrad der Teilnahme, Teilnahmedruck).

- Aber selbst wenn die Teilnahme an einem Behavior Setting komplett freiwillig ist, jede/r willkommen ist und sich alle eine Teilnahme auch leisten können, hängt die Motivation zur Teilnahme stark davon ab, ob eine Person erwartet, ihre individuellen Ziele dort befriedigen zu können, und ob ihre Erwartungen an Partizipationserträge auch bei wiederholter Teilnahme erfüllt werden (vgl. Kaminski 1995, 67). Gerade die erste Teilnahme an einem inklusiven Behavior Setting hängt stark von den kognitiven Bildern ab, die Menschen mit und ohne Beeinträchtigungen voneinander haben und die in die Entstehung zielbezogener Erwartungen einfließen, auch wenn sie noch keine direkten Erfahrungen mit den konkreten Personen in einem Behavior Setting gemacht haben. Direkte Erfahrungen helfen, die kognitiven Bilder voneinander und die eigenen Erwartungen zu korrigieren. Ein Behavior Setting, das auf Freiwilligkeit beruht, wird nur dann inklusiv bestehen können, wenn die Teilnahmeerwartungen von Personen mit und ohne Beeinträchtigungen erfüllt werden.

Nehmen wir wieder inklusive Kunstateliers als Beispiel: In Deutschland sind solche Ateliers in vielen Kommunen nicht vorhanden - und wenn, dann sind sie oft nicht öffentlich ausgeschrieben, sondern finden sich nur in den Verzeichnissen des spezifischen Trägers. Manche potentiellen Teilnehmenden sind auf andere Personen 
angewiesen, die sie auf dieses Angebot aufmerksam machen. Menschen, die nicht selbstständig im Gemeinwesen mobil sein können, würde die Teilnahme durch einen Fahrdienst oder eine Begleitperson erleichtert. Für das süddeutsche inklusive Kunstatelier im obigen Beispiel sind die Zugangsvoraussetzungen bewusst niedrigschwellig. Die Atelierräume wurden barrierearm gestaltet. Die Verbrauchsmaterialien werden finanziert und jemand kann während der Öffnungszeiten kommen und gehen, wann er/sie es will. Da für viele Teilnehmende der äußere Verpflichtungsgrad sehr gering ist, zeugt die gemischte Teilnehmerschaft davon, dass die vielfältigen Partizipationserwartungen und Anspruchsniveaus erfüllt werden.

\subsection{Barrieren der Teilnahme innerhalb von Behavior Settings}

Die Beteiligung am Behavior Setting-Geschehen selbst, das Ausmaß seiner Nutzung, wird zum einen durch setting-spezifische Normen und zum andern durch die Passung des räumlichen Milieus und der Verhaltensobjekte zum Handlungsgeschehen (Synomorphie) reguliert.

- Behavior Setting-Normen: Das Handeln der Teilnehmenden innerhalb eines Behavior Settings wird durch Normen reguliert. Die Übernahme und das Ausfüllen einer bestimmten Rolle in einem Behavior Setting sind mit rollenspezifischen Normen (Rechten und Pflichten) verknüpft. Es wird erwartet, dass eine Person, die eine solche Rolle einnimmt, über die entsprechenden Kompetenzen verfügt oder sie sich aneignet. Kompetenzen umfassen rollenspezifisches Skriptwissen, Fertigkeiten und eine rollenadäquate Handlungsregulation (vgl. ebd., 65f.).

Darüber hinaus gelten für alle Teilnehmenden Normen für ein angemessenes Auftreten und Verhalten im Rahmen des Behavior Settings. Verletzungen von Angemessenheitsnormen fallen auf, auch wenn sie das Programmgeschehen an sich nicht bedrohen. Verletzungen von Angemessenheitsnormen können von den Partizipanten sehr vielfältig verarbeitet (attribuiert) werden und es kann mit ihnen auf sehr unterschiedliche Weise umgegangen werden.

- Synomorphie: Neben den allgemeinen und rollenspezifischen Normen kann die fehlende Passung zwischen dem Handlungsgeschehen, dem räumlichen Milieu und den Verhaltensobjekten eine Barriere darstellen. Die Umgebung und die Verhaltensobjekte sind so zu gestalten, dass sie das Handlungsgeschehen unterstützen, es „leiten“, dass sie im weiten Sinne „lesbar“ sind für die Handelnden. Auch Kommunikationsmittel, dargebotene Informationen und die verwendete Sprache müssen im Sinne der Synomorphie lesbar und verständlich sein.

Bereits in Abschnitt 3 wurden am Beispiel des süddeutschen Kunstateliers die vielfältigen funktionalen Positionen (Rollen), Angemessenheitsnormen und Aspekte der Synomorphie erläutert.

\subsection{Personale Unterstüitzung}

Durch die Unterstützung anderer Personen können manche Barrieren überwunden werden. Unterstützende können die anderen „regulären“ Teilnehmenden 
des Behavior Settings sein oder eine Begleit- oder Assistenzperson, die mit dieser Aufgabe betraut an dem Behavior Setting teilnimmt. Die Unterstützung kann sich auf die Überwindung von Zugangsbarrieren beziehen (z.B. Hilfe bei der Informationsbeschaffung über ein Behavior Setting). Innerhalb eines Behavior Settings kann jemand auch von anderen bei der Ausübung einer Rolle Unterstützung erfahren. Die Unterstützung kann zudem eine korrektive Hilfe für das Einhalten von Angemessenheitsnormen umfassen. Die unterstützenden Personen leisten oft nicht nur instrumentelle und motivationale Hilfe, sondern fungieren kommunikativ als Dolmetscher*in und Ansprechpartner*in, wenn die Person mit Beeinträchtigung sich selbst nicht verständlich machen kann oder die anderen nicht versteht. Aufgabe von Assistenzpersonen kann es darüber hinaus sein, Behavior Settings zu kreieren, an denen dann ein Mensch mit Beeinträchtigung teilnehmen kann. Wird eine Person mit Beeinträchtigung von ihrer Assistenzperson unterstützt, lassen sich die beiden nicht nur als individuelle Teilnehmende begreifen, sondern bilden zugleich eine gemeinsam handelnde Dyade.

\subsection{Grad der Veränderbarkeit von Behavior Settings}

Bislang wurde der strukturierende, den Alltag ordnende, handlungsformende normative Charakter von Behavior Settings betont, an die sich Individuen anzupassen haben. Erwähnt wurde aber auch, dass Teilnehmende im Rahmen von Behavior Settings individuelle Ziele verfolgen, Handlungsfreiräume haben und diese nutzen. Für die Gestaltung inklusiver Behavior Settings ist das Zusammenspiel von überindividuellen Vorgaben und individuellem Teilnehmerhandeln entscheidend: Inwieweit können Behavior Settings so verändert werden, dass die Teilnahme auch mit unterschiedlichen Zielsetzungen und Handlungskompetenzen von Individuen möglich ist? Die mangelnde Veränderbarkeit von Behavior Settings im Sinne einer Anpassung an die individuellen Anforderungen von Teilnehmenden stellt eine gewichtige Barriere dar.

Die Veränderbarkeit von Behavior Settings hängt mit der Flexibilität des Behavior Setting-Programms und dem Autonomiegrad eines Behavior Settings zusammen (vgl. Kaminski 1995).

Ein Behavior Setting-Programm, die Ablauforganisation eines Behavior Settings, kann sehr stark reglementiert bzw. ritualisiert sein oder flexibel ausgeführt werden. Stark reglementierte oder ritualisierte Programmabläufe gehen oft auch mit strikten Normen für angemessenes Verhalten einher - man denke etwa an ein Konzert klassischer Musik für ein erwachsenes Publikum. Behavior Settings mit flexiblem Programm können sich häufig besser auf individuelle Besonderheiten von Teilnehmerhandeln einlassen. Behavior Settings können besser inklusiv gestaltet werden, wenn die Ausgestaltung und Ausführung des Behavior Setting-Programms flexibel gehandhabt werden kann. Allerdings ist zu berücksichtigen, dass die Partizipationserwartungen der verschiedenen Teilnehmerpopulationen gewahrt bleiben müssen und das Behavior Setting insgesamt seine Funktion erfüllt. 
Der Autonomiegrad eines Behavior Settings setzt darüber hinaus der Veränderbarkeit Grenzen. Ein Behavior Setting kann selbstbestimmt, von innen heraus verändert werden oder es wird stark von außen gesteuert durch sog. Authority Systems (Barker \& Associates 1978) oder Exosysteme (Bronfenbrenner 1981). Die organisationale Einbettung und Abhängigkeit von auswärtigen Akteuren spielen eine entscheidende Rolle, gerade in Bezug auf die Verfügbarkeit über Ressourcen, die für das Zustandekommen und Funktionieren des Behavior Settings notwendig sind. Das exemplarisch angeführte inklusive Kunstatelier nutzt die Chance, Freiräume für sehr unterschiedliche künstlerische Vorhaben zu bieten, was den individuellen Erwartungen entspricht. Grundsätzlich lässt sich das Programmgeschehen eines Ateliers für künstlerische Aktivitäten in der Freizeit sehr flexibel gestalten. Das mag z.B. anders sein in einem Chor, in dem hohe Erwartungen an die Qualität des gemeinsamen Gesangs und die Schnelligkeit des Einstudierens (Singen vom Notenblatt) gestellt werden. Die Öffentlichkeitsarbeit des inklusiven Kunstateliers wird allerdings durch die Abhängigkeit von anderen Akteuren begrenzt. Zu ihnen gehören die beiden Anbieter aus der Behindertenhilfe, die das Atelier als tagesgestaltendes Angebot für ihre Klientel organisiert haben und es inklusive der Leitungskraft finanzieren.

\section{Forschungsaufgaben}

Die Teilhabeforschung zielt darauf ab, Barrieren und förderliche Bedingungen zu identifizieren, zu gestalten und umzugestalten. Aus ökologisch-psychologischer Perspektive liegen diesbezügliche Forschungsaufgaben auf unterschiedlichen Ebenen:

\subsection{Analyse der Ökologie von Gemeinwesen bzw. Quartieren}

Mithilfe des Behavior Setting-Konzepts und einer differenzierten Auffassung von Barrieren kann die Reichhaltigkeit eines Gemeinwesens oder Quartiers an inklusiven Settings abgebildet werden. Untersuchungen können die Ökologie von Gemeinwesen bzw. Quartieren für Menschen mit verschiedenartigen unterschiedlichen Beeinträchtigungen vergleichen (Behavior Setting-Inventare; vgl. Barker \& Schoggen 1973). Im Rahmen einer sozialraumorientierten inklusiven Sozialplanung (vgl. Schäper u.a. 2019) können relevante Behavior Settings noch feiner im Hinblick auf Barrieren „adressatenorientiert“ evaluiert und gegebenenfalls umgestaltet werden. Ergebnisse solcher Untersuchungen können zu realitätsnäheren Leitlinien für die Gestaltung inklusiver Gemeinwesen führen.

\subsection{Analyse einzelner Typen von Behavior Settings (Behavior Setting-Genotypen)}

Eine Teilhabeforschung, die Erkenntnisse für die Umweltgestaltung gewinnen will, muss sich stärker auf die Untersuchung einzelner Settingtypen, die Barker (1968) Behavior Setting-Genotypen genannt hat, konzentrieren. Das inklusive 
Kunstatelier in der baden-württembergischen Stadt lässt sich demnach dem Behavior Setting-Genotyp „Kunstatelier für Hobbykünstler*innen“ subsummieren. Ein Beispiel für solche Forschung geben Bigby \& Beadle-Brown (2016) und Clement \& Bigby (2010). Sie fassen Hypothesen und Befunde zusammen, die sich auf die Lebensqualität in sog. Group Homes beziehen, Wohnsettings, in denen drei bis acht Erwachsene mit intellektueller Beeinträchtigung mit 24-Stunden-Assistenz leben. Auch mangels Anwendung theoretischer Konzepte wie des Behavior Settings werden setting-spezifische Erkenntnisse kaum systematisiert. Dabei wäre es hilfreich zu wissen, wie inklusive Settings in den Bereichen Arbeit, Freizeit, Wohnen, Verkehr usw. funktionieren. Auch die Forschungsarbeiten zu sozialen Begegnungen im öffentlichen Raum (vgl. Bigby \& Wiesel 2018) ließen sich mithilfe des Behavior Setting-Konzepts kontextualisieren. Die "Social encounter"Forschung (Bigby \& Wiesel 2011) betont den Wert der einfachen Begegnungen in alltäglichen Geschehenssystemen für die soziale Inklusion und das Zugehörigkeitsgefühl von Menschen mit intellektuellen Beeinträchtigungen. Zu den alltäglichen Geschehenssystemen gehören z.B. Einkaufssituationen, Aufenthalte und Spaziergänge im öffentlichen Raum, Café-Situationen oder Veranstaltungen.

\subsection{Analyse individueller Tages- und Lebensläufe}

Auf der Ebene des Individuums stellt sich, erstens, die Aufgabe mittels der Teilnahme an Behavior Settings sowohl die Aktivitäten als auch die Einbindung einer Person im Gemeinwesen zu erfassen. Das kann grob (Indikator: Teilnahme/Nichtteilnahme) oder feiner durch eine Analyse der Art und des Umfangs der Beteiligung passieren. Ecomaps und Verhaltenskartierungen stellen dar, welche sozial-geografischen Räume von einer Person konkret genutzt werden (vgl. Seifert 2010).

Das Behavior Setting-Konzept erlaubt es, zweitens, realitätsnäher die Anforderungen zu analysieren, die mit einer Teilnahme verbunden sind. Einerseits kann genauer gefasst werden, für was jemand befähigt werden soll bzw. welche Art der Unterstützung er/sie benötigt. Anderseits lässt sich ableiten, wie Behavior Settings barrierefreier umgestaltet werden müssen.

Drittens ist es gewinnbringend, die Teilnahme an Behavior Settings diachron, im Lebenslauf zu betrachten. Gerade bei Menschen mit intellektuellen Beeinträchtigungen bedarf es oft einer langen Phase des Einspielens und der Übung aller Beteiligten, um an einem Behavior Setting in aktiver Rolle partizipieren zu können, etwa in den lokalen Läden aktiv einzukaufen oder Schwimmen zu gehen. Übergänge im Lebenslauf stellen Exklusionsrisiken dar, wenn die eingespielte Teilhabe an konkreten Behavior Settings abbricht und ausgebildete Kompetenzen nicht aufgenommen werden.

\subsection{Analyse von Barrieren und Förderern in Organisationen der Behindertenhilfe}

Viele Menschen mit Beeinträchtigungen sind in ihrer alltäglichen Lebensführung auf professionelle Unterstützung angewiesen. Dienste und Einrichtungen können 
die selbstbestimmte Beteiligung an Behavior Settings jedoch auch behindern. Wie sollten sie konzipiert sein, um die individuelle Teilnahme an Behavior Settings zu fördern? Das Handeln der direkten Assistenzpersonen, denen eine Schlüsselrolle bei der Beteiligung von Menschen mit komplexen Unterstützungsbedarf zukommt, wird nachweislich durch organisationale Bedingungen bestimmt (Bigby \& Beadle-Brown 2016), wie z.B. die Organisationskultur in Wohndiensten, die (Nicht-)Anwendung von Fachkonzepten (wie „Active support“) oder die fachliche Unterstützung durch Leitungskräfte („Practice leadership“). Wieviel Autonomie haben z.B. die an der Basis tätigen Assistenzpersonen und die Klient*innen selbst, um über die Verwendung von Ressourcen zur Gestaltung ihres Wohnalltags zu entscheiden?

\section{Forschungsmethoden}

Eine ökologisch-psychologische Herangehensweise bevorzugt Designs, in denen Barrieren und Förderfaktoren in dem unmittelbaren überindividuellen Handlungskontext untersucht werden. Diese Perspektive wird psychologisch ergänzt um die Individualperspektive, aus der heraus die Teilnahme an Behavior Settings ihre Bedeutung für die individuelle Lebensqualität gewinnt. Die Perspektive größerer Kontexte wie Organisationen oder Gemeinwesen ermöglicht, das Zustandekommen von Barrieren nachzuvollziehen. Auch (sozial-)politische, gesetzliche, administrative Rahmenbedingungen und gesamtgesellschaftliche Werte und Normen tragen zum Verständnis der Entstehung von Barrieren und Förderfaktoren für Behavior Settings bei. Ökologische Psychologie präferiert Mehrebenenanalysen. Forschungsmethodisch lassen sich Barrieren und förderliche Bedingungen am besten durch die direkte Beobachtung des Handlungsgeschehens in den alltäglichen Kontexten erheben. Das kann durch Fremd- oder Selbstbeobachtung, qualitative wie quantitative Beobachtungsverfahren erfolgen (z.B. setting-spezifische Beobachtung, Erfassung von Zeitbudgets und Tagesabläufen, Verhaltenskartierung, Trackingtechniken, Sozialraumbegehungen). Qualitative Interviews und Befragungen ergänzen die direkte Beobachtung. Die so gewonnenen kognitiven Daten sind stets ,situiert“, d.h. in Abhängigkeit von ihrem Entstehungskontext zu interpretieren (vgl. Kaminski 1995, 56f.).

Ein ökologisch-psychologischer Blick, der die Komplexität des alltäglichen Geschehens in seinen Kontexten zu wahren versucht, eröffnet der partizipativen Forschung neue Möglichkeiten. Menschen mit Beeinträchtigungen und andere Akteure werden als Expert*innen ihres Alltags ernst genommen. Zugleich liefert das Behavior Setting-Konzept einen Rahmen, um die Beobachtungen und Erlebnisse unterschiedlicher Teilnehmender zu verorten. Für die Verbesserung selbstbestimmter Teilhabe ist es hilfreich, zwischen der Analyse des Funktionierens von Behavior Settings und dem individuellen Teilnahmeerleben zu unterscheiden. 


\section{Fazit}

Mithilfe des Behavior Setting-Konzepts öffnet sich der Blick der Teilhabeforschung stärker für das Alltagsgeschehen in allen Lebensbereichen und im Gemeinwesen, in denen Behinderung in Erscheinung tritt und sich auswirkt. Barrieren gerade im Hinblick auf Menschen mit intellektuellen und psychischen Beeinträchtigungen ließen sich ökologisch valider formulieren. Auf Anknüpfungspunkte an die internationale Teilhabeforschung (z.B. zu Group Homes und sozialen Begegnungen) wurde hingewiesen. So könnte die Teilhabeforschung auch insgesamt gestaltungsnäher Erkenntnisse generieren, systematisieren und zu sozialen Innovationen beitragen.

\section{Literatur}

Barker, R. (1968): Ecological psychology: concepts and methods for studying the environment of human behavior. Stanford, CA: Stanford University Press.

Barker, R. and Associates (1978): Habitats, environments, and human behavior. San Francisco, CA: Jossey-Bass.

Barker, R. \& Schoggen, P. (1973): Qualities of community life: methods of measuring environment and behavior applied to an American and an English town. San Francisco, CA: Jossey-Bass.

Barker, R. \& Wright, H. (1955): Midwest and its children. New York, NY: Harper and Row.

Bigby, C. \& Beadle-Brown, J. (2016): Improving quality of life outcomes in supported accommodation for people with intellectual disability: What makes a difference? In: Journal of Applied Research in Intellectual Disabilities 31 (2), 1-19.

Bigby, C. \& Wiesel, I. (2018): Using the concept of encounter to further the social inclusion of people with intellectual disabilities: what has been learned? Research and practice in intellectual and developmental disabilities. Online unter: https://doi.org/10.1080/23297018.2018.152817. (Abrufdatum: 21.02.2020).

Bigby, C. \& Wiesel, I. (2011): Encounter as a dimension of social inclusion for people with intellectual disabilities: Beyond and between community presence and participation. In: Journal of intellectual and developmental disability 26(4), 263-267.

Bronfenbrenner, U. (1981): Die Ökologie der menschlichen Entwicklung. Stuttgart: Klett-Cotta.

Clement, T. \& Bigby, C. (2010): Group homes for people with intellectual disabilities. Encouraging inclusion and participation. London, Philadelphia: Jessica Kingsley Publishers.

Day, P. \& Dieckmann, F. (1995): Stadtqualität - nicht nur für junge Gesunde. In: A. Keul (Hrsg.): Wohlbefinden in der Stadt. Umwelt- und gesundheitspsychologische Perspektiven. Weinheim: Beltz/PVU, 198-231.

DIMDI (Deutsches Institut für Medizinische Dokumentation und Information) \& WHO (Weltgesundheitsorganisation) (Hrsg.) (2005): ICF - Internationale Klassifikation der Funktionsfähigkeit, Behinderung und Gesundheit. Köln: Eigenverlag.

Götz, A. (2018): Begegnungsraum Atelier. Das Künstlerische als Katalysator zur Teilhabe. Inklusiv angelegte Kunstateliers im Freizeitbereich und ihre spezifischen Gelingensbedingungen und Teilhabepotentiale. Unveröffentl. Masterthesis, Katholische Hochschule NRW Münster.

Kaminski, G. (2018): Roger G. Barker and Associates: Habitats, environments, and human behavior (1978). In: H.E. Lück, R. Miller \& G. Sewz (Hrsg.): Klassiker der Psychologie. Die bedeutenden Werke: Entstehung, Inhalt und Wirkung. Stuttgart: Kohlhammer, 263-275. 
Kaminski, G. (2008): Das Behavior Setting-Konzept - Entstehungsgeschichte und Weiterentwicklungen. In: E.-D. Lantermann \& V. Linneweber (Hrsg.): Grundlagen, Paradigmen und Methoden der Umweltpsychologie. Göttingen: Hogrefe, 333-376.

Kaminski, G. (2002): Roger G. Barker. In: H. Lück \& R. Miller (Hrsg.): Illustrierte Geschichte der Psychologie. Weinheim: Beltz, 194-198.

Kaminski, G. (1995): Behinderung in ökologisch-psychologischer Perspektive. In: J. Neumann (Hrsg.): „Behinderung“. Von der Vielfalt eines Begriffs und dem Umgang damit. Tübingen: Attempto, 44-74.

Kaminski, G. (1985): Ökopsychologische Beiträge zur Verbesserung der Lebensqualität geistig behinderter Menschen. In: E. Wacker \& J. Neumann (Hrsg.): Geistige Behinderung und soziales Leben. Frankfurt/M.: Campus, 144-158.

Kaminski, G. (Hrsg.) (1976): Umweltpsychologie. Perspektiven, Probleme, Praxis. Stuttgart: Klett-Cotta.

Lewin, K. (1943): Forces behind food habits and methods of change. In: The Committee on Food Habits (Ed.): The problem of Changing Food Habits. Report. Washington, D.C.: National Research Council (NRC), National Academy of Sciences, 35-65.

Schäper, S., Dieckmann, F., Rohleder, C., Rodekohr, B., Katzer, M. \& Frewer-Graumann, S. (2019): Inklusive Sozialplanung für Menschen im Alter. Stuttgart: Kohlhammer.

Seifert, M. (2010): Kundenstudie. Bedarf an Dienstleistungen zur Unterstützung des Wohnens von Menschen mit Behinderung. Berlin: Rhombos-Verlag.

Weischer, C. \& Gehrau, V. (2017): Die Beobachtung als Methode in der Soziologie. Konstanz: UVK Verlagsgesellschaft. 
Markus Schäfers und Viviane Schachler

\section{Barrieren erfragen - Herausforderungen der empirischen Erfassung von Barrieren im Rahmen standardisierter Interviews}

Wie lassen sich Barrieren forschungsmethodisch erfassen? Wie lässt sich Barrierefreiheit messen? Welche Erkenntnisse über die Beschaffenheit von Umwelten lassen sich daraus gewinnen? Für empirische Sozialforschung ist es eine methodische Herausforderung, gestaltete Umwelten systematisch und differenziert darauf hin zu prüfen, ob sie für Menschen mit Beeinträchtigungen Barrieren bilden. Dies gilt in besonderer Weise für standardisierte Befragungen. Gemeinhin gilt die Methode der Befragung als „Königsweg“ der Sozialforschung (vgl. Diekmann 2004, 371), wenn es darum geht, Einschätzungen und Bewertungen aus der Subjektperspektive zu erheben. Insofern erscheint diese Methode auch bei der Erfassung von Barrieren naheliegend. Welche konkreten Zugangswege im Rahmen standardisierter Befragungen beschritten werden können und welche Probleme damit einhergehen, diskutiert der vorliegende Beitrag. Dabei werden kontrastierend zwei prinzipiell mögliche Verfahrensweisen dargestellt und im Hinblick auf Messvorteile und -risiken diskutiert. Hintergrund dieser Betrachtung bildet die "Repräsentativbefragung zur Teilhabe von Menschen mit Behinderungen in Deutschland" (vgl. Schröder u.a. 2017). Im Auftrag des Bundesministeriums für Arbeit und Soziales (BMAS) führt das Institut für angewandte Sozialwissenschaft (infas Bonn) in Kooperation mit der Hochschule Fulda diesen Teilhabesurvey durch (Laufzeit 2017-2021). In drei Teilstudien, die unterschiedliche Lebenskontexte der Befragungsgruppe berücksichtigen, werden 22.000 Menschen mit Beeinträchtigungen befragt. Alle wichtigen Lebensbereiche werden daraufhin untersucht, wo Teilhabe gelingt und wo nicht. Das schließt auch die Barrierefreiheit verschiedener Lebenskontexte ein (vgl. z.B. BMAS 2017, 221). Dies gibt Anlass zu den grundlegenden Fragen, wie Barrieren im Rahmen standardisierter Befragungen begrifflich gefasst, operationalisiert und erhoben werden können.

\section{Ausgangslage und Verständnis von Barrieren}

Validierte standardisierte Erhebungsinstrumente zur Barrierefreiheit, die im Rahmen einer Bevölkerungsumfrage Anwendung finden könnten, liegen für den deutschsprachigen Raum bisher noch nicht vor. Zwar gibt es Instrumente, die 
das Konstrukt zu messen versuchen, diese beschränken sich jedoch auf Teilaspekte des mehrdimensionalen Konzepts oder sind - wie im Folgenden noch gezeigt wird - im Lichte klassischer Gütekriterien quantitativer Forschung eher kritisch zu bewerten.

Für die Konstruktion eines Instruments, das Barrieren bzw. Barrierefreiheit misst, stellt das bio-psycho-soziale Behinderungsmodell der ICF (WHO 2001) eine wichtige konzeptionelle Grundlage dar. Barriere im Sinne der ICF ist ein relationaler Begriff (vgl. Kastl 2017, 95ff.). Er beleuchtet das Verhältnis zwischen Körperfunktionen/-strukturen, Aktivitäten/Teilhabe bzw. Partizipation und Umweltfaktoren: Jemand mit einer bestimmten körperlichen Disposition wird durch etwas bei etwas behindert. Nicht eine bestimmte Eigenschaft einer Umwelt stellt an sich eine Barriere dar (z.B. der Bordstein), sondern erst dann, wenn jemand mit bestimmten Beeinträchtigungen in spezifischen Situationen auf diese Umwelt trifft (z.B. eine körperlich beeinträchtigte Person im Rollstuhl) mit dem Ergebnis, dass diese Person eine Aktivität nicht ausüben bzw. nicht am gesellschaftlichen Leben teilhaben kann. Das heißt, dass die Beurteilung der Barrierefreiheit einer Umwelt nur vorgenommen werden kann mit Bezug auf (mehr oder minder) konkrete körperliche, psychische oder geistige Beeinträchtigungen, Aktivitäten sowie ggf. weitere Umweltfaktoren. Methodologisch gedacht kann es also nicht hinreichend sein, ausschließlich bestimmte Merkmale einer Umwelt abzubilden. Vielmehr müssen Methoden in der Lage sein, die Person-Umwelt-Relation zu erfassen.

Mit Blick auf bestehende Erhebungsinstrumente (vgl. Abschnitt 2) lassen sich zwei Denkrichtungen differenzieren, die Barrieren und Barrierefreiheit unterschiedlich begreifen:

- Zum einen kann Barrierefreiheit objektbezogen, von den Umweltbedingungen ausgehend, gedacht werden. Hiernach ist eine Infrastruktur dann barrierefrei, wenn bestimmte strukturelle Bedingungen erfüllt sind. Indiziert wird dies über nicht vorhandene Barrieren. Ein Subjektbezug ist nicht gegeben. Im Zuge der Operationalisierung von Barrierefreiheit werden objektive Umweltanforderungen fokussiert. Auch wenn ein konkreter Personenbezug fehlt, sind in diesen Anforderungen implizite Bezüge zu Gruppen von Menschen mit Beeinträchtigungen oder Unterstützungsbedarfen enthalten. Von den angenommenen Beeinträchtigungen oder Bedarfen wird auf Anforderungsindikatoren der Umwelt geschlossen (siehe hier auch die Darstellung zur Zugänglichkeit/Accessibility von Welti in diesem Band). Dies lässt sich als typisierendes, objektbezogenes Vorgehen charakterisieren, wie dies auch der Ansatzpunkt in Normen des Deutschen Instituts für Normung (DIN-Normen) bildet (vgl. Rebstock in diesem Band).

- Zum anderen kann Barrierefreiheit subjektbezogen, von Aktivitäten ausgehend, gedacht werden. Dieser Ansatz rückt die Frage in den Vordergrund, welche Aktivitäten nicht ausgeübt werden können oder welche Teilhabedimensionen eingeschränkt sind aufgrund von Bedingungen der Umwelt. Der Situationsbezug 
steht im Vordergrund. Betrachtet wird, mit welchen Umweltbedingungen sich Personen situativ konfrontiert sehen, die Aktivitäten und Teilhabe verhindern. Anknüpfungsfähig ist hier zudem die Weiterführung, dass die einschränkende Wirkung von Barrieren überwunden und überdies in Unterstützungsfaktoren umgewandelt werden kann (vgl. Hirschberg in diesem Band). Im Gegensatz zum infrastrukturellen Ansatz wird bei dieser Denkrichtung stärker die Perspektive des Subjekts eingenommen, in dem ausgehend von einer Lebenssituation oder einem Handlungskontext auftretende Barrieren identifiziert werden.

Im Folgenden werden diese beiden Zugangsweisen durch Beispiele aus sozialwissenschaftlichen Erhebungsinstrumenten veranschaulicht. Damit lässt sich zugleich auf verschiedene methodologische Herausforderungen verweisen, die bei der Erhebung von Barrieren im Rahmen standardisierter Befragungen auftreten.

\section{Diskussion vorliegender Ansätze zur Operationalisierung von Barrierefreiheit im Rahmen standardisierter Befragungen}

\subsection{Der objektbezogene Ansatz: Erfassung von Barrieren über Umweltbedingungen}

Ein erstes Beispiel verdeutlicht den objektbezogenen Ansatz in einer allgemeinen Variante: Die Befragten werden nach ihrer Einschätzung der Barrierefreiheit typischer Orte in ihrem Umfeld gebeten. Der Ausschnitt aus einer schriftlichen Befragung in Abbildung 1 zeigt diesen Ansatz. Das Beispiel ist einer hessenweiten Befragung entnommen (vgl. Rambausek 2017), die u.a. Einschätzungen zu 18 Orten im Lebensumfeld mithilfe einer dreistufigen Ratingskala (gut/mittelmäßig/schlecht) umfasst.

\begin{tabular}{|lccc} 
Wie barrierefrei ist Ihr/lhre & Gut & Mittelmäßig & Schlecht \\
\hline Apotheke & $\square$ & $\square$ & $\square$ \\
\hline Arzt & $\square$ & $\square$ & $\square$ \\
\hline Bank & $\square$ & $\square$ & $\square$ \\
\hline Bibliothek & $\square$ & $\square$ & $\square$
\end{tabular}

Abb. 1: Allgemeine Bewertungen der Barrierefreiheit von Orten im Lebensumfeld (eigene Anfertigung nach Rambausek 2017, 477)

Dieser Ansatz ist zeiteffizient. Die Itemformulierungen sind kurz und prägnant. Allerdings beinhalten sie einen großen Spekulationsspielraum. Erstens bleibt der Personenbezug unklar: Soll angeben werden, ob die Orte für mich oder für andere (grundsätzlich) barrierefrei sind? Zweitens setzt die Frage voraus, dass sich die befragte Person bei der Antwort auf konkrete Orte, Angebote bzw. Institutionen bezieht. Offen bleibt, wie in der Beantwortung vorzugehen ist, wenn bei alltäglichen 
Besorgungen mehrere Orte eines Typs besucht werden (welche Bank ist gemeint, wenn ich mein Geld in verschiedenen Banken abhebe?), die Angebote im Umfeld nicht vorhanden sind oder gar nicht genutzt werden (wollen), sodass keine Erfahrungswerte zur Barrierefreiheit vorliegen. Drittens bleibt unklar, was mit barrierefrei im Speziellen gemeint ist. So bleibt bei dieser Spielart der Operationalisierung die Definition von Barrierefreiheit völlig dem Deutungsraum der Befragten überlassen. Zwar lässt sich mit Blick auf den Untersuchungskontext dieses konkreten Beispiels (Befragtengruppe der Menschen mit einer anerkannten Schwerbehinderung und dem Merkzeichen außergewöhnliche Gehbehinderung; vgl. Rambausek 2017, 378) eine rollstuhlgerechte Umgebung assoziieren, dennoch bleibt dies spekulativ. Ergebnisse regelmäßiger Online-Umfragen in ausgewählten Kommunen, die ein "Stimmungsbild in der Gesellschaft zum Thema Barrierefreiheit" (Aktion Mensch 2017, 1) erzielen sollen, verweisen etwa darauf, dass die alltagsweltlichen Assoziationen zu Barrierefreiheit differieren und zeitlichen Veränderungen unterliegen (vgl. ebd., 3). Werden Itemformulierungen in der dargestellten generellen Form verwendet, sind Abschläge der Reliabilität zu erwarten; auch die Inhaltsvalidität ist fraglich (vgl. Bortz \& Döring 2006, 200), da unklar ist, ob den Antworten ein gemeinsames Verständnis von Barrierefreiheit zugrunde liegt.

Um nun den vorliegenden Spekulationsspielraum einzugrenzen, kann Barrierefreiheit zugespitzt und beispielsweise über vorliegende Hindernisse beim Besuch bestimmter Orte erfragt werden. Dergestalt ist die Abfrage von Barrierefreiheit in der „Vorstudie für eine Repräsentativbefragung zur Teilhabe von Menschen mit Behinderung(en)“ (Schröttle \& Hornberg 2014a) aufgebaut. Diese den Teilhabesurvey vorbereitende Auftragsstudie lieferte einen ersten Instrumentenentwurf, der unter der Überschrift „Barrierefreiheit im öffentlichen Raum und im öffentlichen Verkehr“" (ebd. 2014b, 72) die in Abbildung 2 dargestellte Frageformulierung beinhaltet.

Gibt es für Sie Einschränkungen, Hindernisse oder Probleme im öffentlichen Raum, zum Beispiel bei der Nutzung von Bahnhöfen, Verkehrsmitteln und öffentlichen Gebäuden?
$\square$ ja
$\square$ nein
weiß nicht
keine Angabe

Abb. 2: Erfassung von Barrieren im öffentlichen Raum (eigene Anfertigung nach Schröttle \& Hornberg 2014b, 72)

Mit dieser Vorgehensweise soll indiziert werden, dass bei einer ,ja-Antwort“ Barrieren bestehen, die in Folgefragen weiter spezifiziert werden, z.B. im Hinblick auf verschiedene Orte und Arten von Barrieren (z.B. öffentliche Gebäude als „Kulturelle Einrichtungen [Museum, Theater, Kino] " und "fehlende[r] Kompetenz in Deutscher Gebärdensprache" ebd., 73). Andersherum indiziert eine „nein-Antwort“, dass Barrierefreiheit gegeben ist. 
Im Unterschied zum vorherigen Beispiel stellt diese Fragekonstruktion einen Personenbezug („Gibt es für Sie ...?“) und einen Situationsbezug her (Nutzung von Örtlichkeiten bzw. Verkehrsmitteln). Dieser Ansatz kommt dann an Grenzen, wenn es vorgelagerte Barrieren gibt, welche die Nutzung dieser Örtlichkeiten bereits im Vorfeld verhindern (z.B. ein öffentliches Gebäude, das nicht über den ÖPNV erreichbar ist). Wie im vorangegangenen Beispiel bleibt das Problem, dass in solchen Fällen die Befragten vorhandene Hindernisse der Örtlichkeit mangels fehlender Erfahrungswerte selbst gar nicht beurteilen können. So schlussfolgert eine Studie über Barrieren und Barrierefreiheit im Kontext kognitiver Beeinträchtigungen, dass Barrieren nur erfahrbar sind, wenn vorgelagerte Zugänge gegeben sind. Dies wird am Beispiel von gastronomischen Einrichtungen erläutert:

„Das Fehlen von Speisekarten in Leichter Sprache in einem Restaurant oder Café wird erst dann als Barriere erfahrbar, wenn die Lebensbedingungen der potenziellen NutzerInnen einen Restaurant- oder Cafébesuch überhaupt erst möglich machen." (Trescher 2018, 139)

Einen weiteren Ansatz verfolgt die Studie „Beeinträchtigt studieren“ des Deutschen Studentenwerks (2012). Fehlende Barrierefreiheit wird hier örtlich und sächlich eingegrenzt durch die Erfassung baulicher Eigenschaften bestimmter Orte (an Hochschulen). Wie Abbildung 3 zeigt, sind in der Interviewführung Fragen vorgelagert, die ermitteln, ob bei den Befragungspersonen spezielle beeinträchtigungsbedingte Gestaltungsbedarfe in Hinblick auf elf Indikatoren (sowie einer offenen Antwortoption) gegeben sind. Wenn dies der Fall ist, folgen Bewertungen darüber, inwieweit die Bedarfe an der Hochschule bereits erfüllt werden, was mit einer dreistufigen Skala gemessen wird. Nur wenn ein indizierter Bedarf an barrierefreien Umwelten als teilweise oder nicht ausreichend erfüllt eingestuft wird, sieht die Filterführung die Abfrage von Bereichen der barrierefreien baulichen Grundausstattung/Zugänglichkeit vor.

Diese Fragestruktur spitzt mangelnde Barrierefreiheit auf die eigene Person, eine bauliche Zugänglichkeit und zehn typische Orte des Studierendenlebens zu, die für die Befragten zudem wichtig sein sollen. Mit dieser Anpassung an den Forschungsgegenstand und die Befragtengruppe gelingt es der personalisierten Online-Befragung von Studierenden von 160 Hochschulen (vgl. ebd., 275ff.), den abstrakten Begriff Barrierefreiheit einzugrenzen und Teildimensionen des Konstrukts zu messen. Im Ergebnis liegen zuverlässige Angaben zu ausgewählten infrastrukturellen (primär baulichen und ausstattungsbedingten) Arten von Barrieren an Hochschulen vor sowie Angaben zu örtlichen Bereichen, an denen diese Barrieren bestehen, womit wiederum Rückschlüsse auf notwendige Verbesserungsbedarfe möglich sind. 


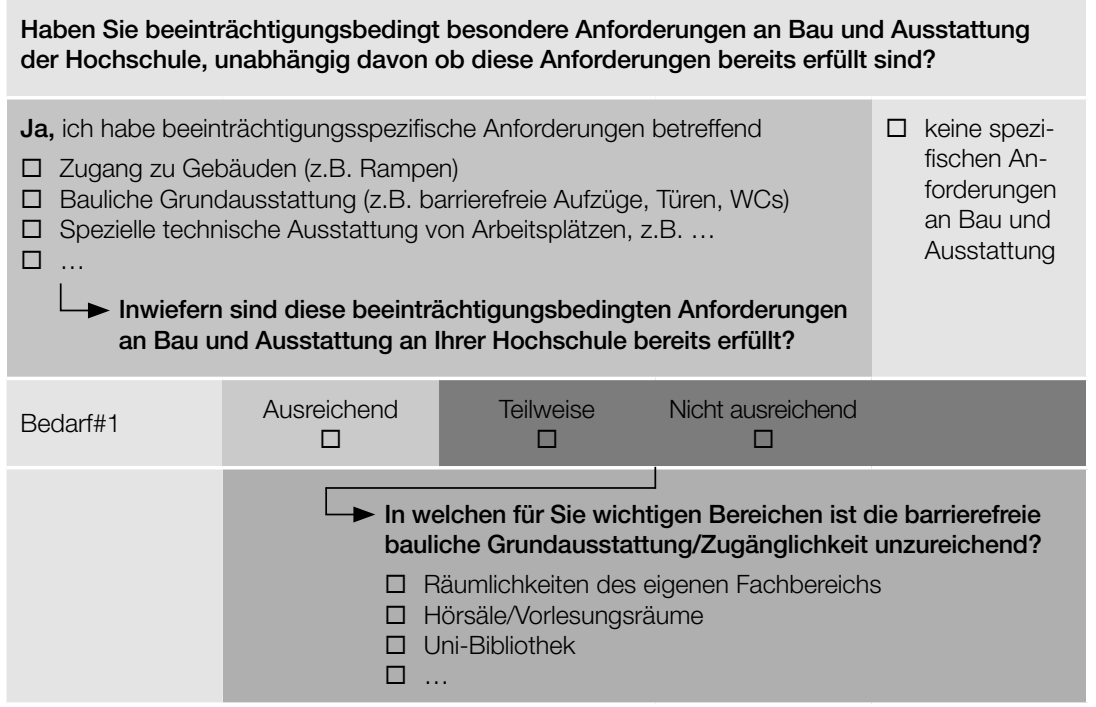

Abb. 3: Spezielle örtliche Erfassung von Komponenten einer barrierefreien Infrastruktur (eigene Anfertigung nach Deutsches Studentenwerk 2012, 305f.)

An Grenzen stößt diese zielgerichtete Vorgehensweise (Abfrage von vorliegenden Bedarfen an barrierefreie Umwelten; Einschätzung zur bisherigen Deckung; Abfrage der Bereiche bei ungedecktem Bedarf) im Rahmen einer großen Bevölkerungsumfrage, bei der sehr heterogene Lebenslagen der Zielpopulation zu erwarten sind. Hier zeigt sich ein massives Auswahlproblem. Die Fragebogenkonstruktion setzt zwingend eine Begrenzung des „Itemuniversums“ (Guttmann 1950 nach Schnell u.a. 2013, 123) voraus: Aus einem Pool möglicher Indikatoren müssen die passenden ausgewählt werden. Je größer die Themenfülle der Befragung ist und je heterogener die abzudeckenden Lebenssituationen und -kontexte sind, desto schwieriger fällt die Bestimmung der Indikatoren - was auf Barrierefreiheit in besonderem Maße zutrifft, da das Itemuniversum unbekannt ist. Es stellen sich Fragen der Inhaltsvalidität (vgl. Bortz \& Döring 2006, 200), also Fragen danach, wie eine hohe inhaltliche und möglichst umfassende Deckung zwischen den gemessenen Aspekten und dem zu messenden Konstrukt Barrierefreiheit erreicht werden kann. So manifestiert sich Barrierefreiheit nicht nur durch physische bzw. räumliche Indikatoren. Barrierefreiheit umfasst auch Aspekte einer angemessenen Nutzbarkeit, z.B. macht es im Studienalltag für Rollstuhlnutzende durchaus einen Unterschied, ob ein Universitätsgebäude ebenerdig oder über einen entfernten Hintereingang zugänglich ist und wie schnell sich ein Raumwechsel realisieren lässt. 
Nach der Nutzbarkeit von Orten bzw. des Transports/Nahverkehrs wird z.B. in der Kurzfassung des Model Disability Surveys der WHO/The World Bank (o.J.) gefragt (siehe Abbildung 4). Den Items liegt jeweils eine fünfstufige Skala (von „very easy“ bis „very hard“) zugrunde.

\begin{tabular}{|c|c|c|c|c|c|c|c|}
\hline $\begin{array}{l}\text { I am going to ask you some general questi- } \\
\text { ons about your environment and your social } \\
\text { relationships. I want you to answer the follo- } \\
\text { ing questions on a scale from } 1 \text { to } 5 \text {, where } \\
1 \text { means very easy and } 5 \text { means very hard. }\end{array}$ & $\begin{array}{c}1 \\
\text { Very } \\
\text { easy }\end{array}$ & 2 & 3 & 4 & $\begin{array}{l}5 \\
\text { Very } \\
\text { hard }\end{array}$ & $\begin{array}{c}8 \\
\text { Don't } \\
\text { know }\end{array}$ & $\begin{array}{l}98 \\
\text { Not } \\
\text { appli- } \\
\text { cable }\end{array}$ \\
\hline $\begin{array}{l}\text { Do places where you socialize and engage in } \\
\text { community activities make it easy or hard for } \\
\text { you to do this? }\end{array}$ & & & & & & & \\
\hline $\begin{array}{l}\text { Do the shops, banks and post office in your } \\
\text { neighborhood make it easy or hard for you to } \\
\text { use them? }\end{array}$ & & & & & & & \\
\hline $\begin{array}{l}\text { Does the transportation you need or want to } \\
\text { use make it easy or hard for you to use it? }\end{array}$ & & & & & & & \\
\hline $\begin{array}{l}\text { Does your dwelling, including the toilet, make } \\
\text { it easy or hard for you to live there? }\end{array}$ & & & & & & & \\
\hline
\end{tabular}

Abb. 4: Einschätzungen zur Benutzbarkeit der Infrastruktur (eigene Anfertigung nach WHO/The World Bank o.J., 1)

Diese Frageart scheint nun im Hinblick auf eine internationale Orientierung und mögliche Vergleichbarkeit zunächst vielversprechend. In der Anwendung stellt sich jedoch ein sprachliches Übersetzungsproblem: Die Formulierung „Macht Ihre Umwelt es Ihnen leicht/schwer ...?“ im Sinne einer direkten Übersetzung erscheint im Deutschen ungebräuchlich und künstlich. Mögliche sprachlich angepasste Alternativen wären: „Haben Sie Probleme dabei, die Post zu besuchen, oder gibt es für Sie Hindernisse, wenn Sie in die Bank gehen?" Diese Formulierung kann wiederum verschiedene Assoziationen hervorrufen, die über das Begriffsfeld von Barrierefreiheit hinausreichen, z.B. wenn im ländlichen Raum infrastrukturelle Versorgungsprobleme oder Rückbauten im Bereich des Post-/Bankwesens oder ÖPNV bestehen. Offen bleibt bei dieser Abfrageform auch, wodurch die Umwelt eine Aktivitätsausführung erschwert, also welche Eigenschaften der Umwelt im Einzelnen ausschlaggebend sind.

Was hier mit der Dimension der Nutzbarkeit jedoch bereits anklingt, ist eine Verknüpfung von Umweltbedingungen mit Aktivitäten, womit sich anfragen lässt, ob eine Messung von Barrieren besser funktioniert, wenn diese gezielt von Aktivitäten ausgehend erfasst wird. 


\subsection{Der subjektbezogene Ansatz: Erfassung von Barrieren über Aktivitäten}

Eine Möglichkeit, um Aktivitätsbeeinträchtigungen durch Barrieren zu erfassen, ist in der bereits erwähnten Vorstudie des Teilhabesurveys umgesetzt. Unter der Überschrift „Barrierefreier Zugang zu Medien und Informationen (Barrieren)“ wird gezielt nach Einschränkungen bei fünf Aktivitäten der Mediennutzung gefragt (siehe Abbildung 5).

\begin{tabular}{|c|c|c|c|c|c|}
\hline $\begin{array}{l}\text { Gibt es für Sie Einschränkungen aufgrund Ihrer Behinde- } \\
\text { rungen oder Beeinträchtigung bei der Nutzung folgender } \\
\text { Medien? }\end{array}$ & ja & nein & $\begin{array}{l}\text { nutze } \\
\text { ich } \\
\text { nicht }\end{array}$ & $\begin{array}{l}\text { weiß } \\
\text { nicht }\end{array}$ & $\begin{array}{l}\text { keine } \\
\text { An- } \\
\text { gabe }\end{array}$ \\
\hline Beim Lesen von Büchern, Zeitschriften und Zeitungen & $\square$ & $\square$ & $\square$ & $\square$ & $\square$ \\
\hline $\begin{array}{l}\text { Bei der Nutzung des Internets } \\
\ldots\end{array}$ & $\square$ & $\square$ & $\square$ & $\square$ & $\square$ \\
\hline \multicolumn{6}{|c|}{$\begin{array}{l}\text { Was benötigen Sie, um (Informations-)Medien ohne Einschrän- } \\
\text { kungen nutzen zu können? } \\
\text { (Frage offen stellen und Antworten zuordnen) }\end{array}$} \\
\hline $\begin{array}{l}\square \text { Einfache/Leichte Sprache } \\
\square \text { Visuelle Elemente (Bilder, Symbole, Fotos) } \\
\square \text { Text statt Ton (Untertitel, Skript) } \\
\square \quad \text {... }\end{array}$ & & & & & \\
\hline
\end{tabular}

Abb. 5: Erfassung von Barrieren bei der Mediennutzung (eigene Anfertigung nach Schröttle \& Hornberg 2014b, 77)

Wird eines der Items bejaht, folgt in der Filterführung die offene Abfrage: „Was benötigen Sie, um (Informations-)Medien ohne Einschränkungen nutzen zu können?" (Schröttle \& Hornberg 2014b, 77). Die frei formulierten Antworten der befragten Person soll der/die Interviewer*in in zehn verschiedene Kategorien einordnen. Diese Vorgehensweise setzt ein Wissen über Assistive Technologien (vgl. Klein in diesem Band) voraus und ist somit voraussetzungsvoll. Dennoch scheint die Zugangsweise, Barrieren über die Ausführung von speziellen Aktivitäten zu messen, prinzipiell geeignet, um (indirekt) Auskunft über barrierefreie Nutzungen zu erzielen. Unklar bleibt die Interpretation der Antwortoption "nutze ich nicht": Bedeutet diese, dass eine Aktivität nicht ausgeübt wird, z.B. weil dies die befragte Person nicht will („,nutze ich nicht, weil die Aktivität für mich irrelevant ist“), oder dass Barrieren die Aktivitätsausführung grundsätzlich verhindern („nutze ich nicht, weil ich keinen Zugang habe")?

Ein weiterer, daran anknüpfender Ansatz erlaubt diese Differenzierung. Abbildung 6 zeigt ein Beispiel aus der Studie "Pretest Befragung in Einrichtungen“ (vgl. Schäfers u.a. 2016a), einer weiteren Vorstudie des Teilhabesurveys. Im Abschnitt „Freizeit und kulturelle Teilhabe“ wird zunächst danach gefragt, ob eine Freizeitaktivität ausgeführt wird. Unabhängig von der Ausübung oder Nicht-Ausübung der Aktivität wird nachgefragt, ob die Person sich eine Veränderung wünscht. 
Anschließend wird im Falle eines Wunsches nach (häufigerer) Ausübung der Aktivität die Frage nach den Hinderungsgründen gestellt.

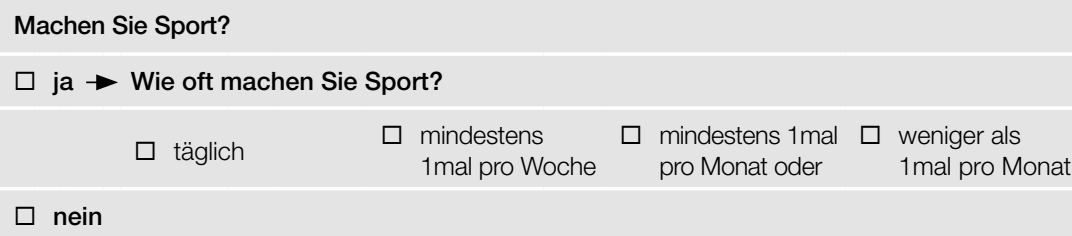

Möchten Sie gerne (mal/häufiger) Sport machen?

ja $\rightarrow$ Was hindert Sie daran? (offen stellen und Antworten zuordnen)

$\llcorner\square$ zu bequem/kann mich nicht aufraffen

$\square$ meine Beeinträchtigung/Behinderung

$\square$ zu wenig/keine Zeit

$\square$ kein/e Freizeitpartner/in, der/die mitmacht

$\square$ kein Geld/zu teuer

$\square$ in meiner Gegend nicht vorhanden

$\square$ keine Assistenz vorhanden (auch Fahrdienst)

$\square$ Barrieren hindern mich daran (z.B. baubedingte Zugänglichkeit)

$\square \ldots$

nein

Abb. 6: Erfassung von Barrieren als Ursachen von fehlenden Aktivitäten (eigene Anfertigung nach Schäfers u.a. 2016b, 35f.)

Unter den Antwortoptionen finden sich solche, die nichts mit Barrieren zu tun haben (z.B. „zu wenig/keine Zeit"), und welche, die auf Barrieren hinweisen (z.B. „Barrieren hindern mich daran"). Im Sinne der ICF wird in der Frage die Verknüpfung zwischen den verschiedenen Komponenten des bio-psycho-sozialen Modells hergestellt: zwischen Aktivitäten (in der Freizeit), Körperfunktionen/ -strukturen („meine Beeinträchtigung/Behinderung“), personbezogenen Faktoren (z.B. „zu bequem“) und Umweltfaktoren (z.B. „kein Geld“, „Barrieren“, „Einstellungen anderer").

Die Beantwortung dieser Fragen erfordert jedoch von der befragten Person ein gewisses Abstraktions- und Reflexionsvermögen, weil sie ggf. kontrafaktische Informationen geben muss: Warum tue ich etwas nicht, obwohl ich es eigentlich tun möchte? Dies kann insbesondere bei der Befragung von Menschen mit kognitiven Beeinträchtigungen herausfordernd sein. Forschungsmethodisch ergibt sich die Situation, dass die Frage- und Aufgabenstellung "mit bestimmten Merkmalen der Befragten systematisch konfundiert sein [kann], ohne dass es eine Möglichkeit gibt, dies nach Beendigung der Umfrage festzustellen." (Faulbaum u.a. 2009, 56f.) So sind Personen in unterschiedlichem Maße darin geübt und dazu in der Lage, die eigene Situation schnell zu erfassen und in eine sofortige reflektierte Antwort zu 
überführen, womit vor dem geschilderten Themenhintergrund eine Verzerrung des Messergebnisses („Bias“) angelegt sein kann. In der Methodologie gilt eine solche Konfundierung („Störvariable“) als Gefährdung der internen Validität (vgl. ebd.), d.h., die erzielten Ergebnisse können nicht eindeutig für ermittelte Zusammenhänge mit Barrieren stehen (vgl. Bortz \& Döring 2006, 53). Zudem ist die Liste der Antwortoptionen lang und dennoch nicht abschließend. Das bedeutet, dass sie einerseits in der Interviewführung zeitintensiv ist und erschöpfend wirken kann, andererseits Zweifel aufkommen lässt, ob sich die individuellen Gründe in eine überschaubare Anzahl von Kategorien überführen lassen, was wiederum die inhaltliche Validität der Messung in Frage stellt.

\section{Zusammenfassung und Schlussfolgerungen}

Zur Erfassung von Barrierefreiheit im Rahmen standardisierter Befragungen liegen für den deutschsprachigen Raum keine bewährten Instrumente vor. Vorhandene Befragungen erfassen lediglich Teildimensionen des komplexen Konstrukts oder zeigen Schwachstellen bei der Einhaltung quantitativer Gütekriterien. Auch der Rückgriff auf internationale Instrumente scheint nicht praktikabel, da diese in der Übersetzung an Präzision verlieren. Zwei vorgestellte Zugangswege bieten potenzielle Möglichkeiten, sich dem Konstrukt im Einklang mit dem Behinderungsmodell der ICF anzunähern:

- die Erfassung von Barrieren über Umweltbedingungen (objektbezogener Ansatz)

- die Erfassung von Barrieren über Aktivitäten (subjektbezogener Ansatz)

Barrieren über Umweltbedingungen zu erfassen, entspricht wohl am ehesten dem alltagsweltlichen Verständnis von Barrieren: Barrieren werden an Eigenschaften der gestalteten Umwelt festgemacht, z.B. Stufen und Treppen, zu schmale Wege und Türen (für Rollstühle), nicht erreichbare Bedienelemente oder nicht zugängliche Informationen.

Diese Umwelteigenschaften können aus empirischer Sicht vergleichsweise einfach erhoben werden. Die Operationalisierung trifft jedoch zum einen auf ein Definitionsproblem: Welche Eigenschaft genau macht aus einer gestalteten (z.B. baulichen) Umwelt eine Barriere? Hier lässt sich auf die Normgebung zurückgreifen (z.B. DIN). Das Wissen über Barrieren und die Normgebung sind jedoch im Hinblick auf die Anforderungen für Menschen mit körperlichen oder Sinnesbeeinträchtigungen weiter vorangeschritten und manifester als im Hinblick auf Menschen mit kognitiven oder psychischen Beeinträchtigungen, die eher mit latenten Barrieren konfrontiert werden. Zum anderen sieht sich die Operationalisierung mit einem Auswahlproblem konfrontiert, da das Universum möglicher Barrieren grenzenlos erscheint. Dieses Problem wirkt umso schwerer, je mehr Lebensbereiche und -kontexte erforscht werden und je heterogener die Zielpopulation ist. Bei einer Quer- 
schnitts- oder Längsschnittuntersuchung der Lebenslagen von Menschen mit verschiedenen Beeinträchtigungen in einem standardisierten Design erscheint hier nur eine ausschnitthafte Betrachtung von Barrieren und Barrierefreiheit umsetzbar.

Ein weiteres Problem wiegt noch schwerer: In der objektbezogenen Operationalisierung von Barrieren verbergen sich ,verkleidete Normen der Gesellschaft“ (Kastl 2017, 49), welche die Bedarfe des Subjekts außen vorlassen können. Eine Wohnung gilt gemäß geltenden Bauordnungen dann als barrierefrei oder rollstuhlgerecht, wenn sie ebenerdig oder mit einem Aufzug erreichbar ist, gewisse Türmaße hat oder einen mit einem Rollstuhl unterfahrbaren Waschtisch usw. Im konkreten Fall einer gehbeeinträchtigten Person wäre aber u.U. ein rutschfester Boden entscheidend für die Frage der Alltagsbewältigung. Der objektbezogene Ansatz setzt also eine Typisierung der Bedarfe voraus, die im Einzelfall nicht zutreffend und auch nicht teilhaberelevant sein muss.

Barrieren über Aktivitäten zu erfassen, geht demgegenüber vom Subjekt aus: Bei welchen Aktivitäten einer Person wirken Hindernisse in der Umwelt dergestalt, dass diese Aktivitäten nicht oder nur eingeschränkt ausgeübt werden? Der subjektbezogene Ansatz lässt sich handlungstheoretisch fundieren: Um eine Handlung auszuführen, muss die Person erstens dafür objektiv leistungsfähig genug sein. Zweitens muss die Person die Handlung auch durchführen wollen. Drittens, und das stellt die Verbindung zur Barrierefreiheit her, müssen die äußeren Umstände es der Person ermöglichen, ihre Leistungsfähigkeit in die gewollte Handlung umzusetzen (vgl. Nordenfelt 2003; Schuntermann 2004, 21f.).

Um Hindernisse der Umwelt von anderen Gründen, eine Aktivität nicht auszuüben oder ausführen zu können, zu unterscheiden, muss ein Erhebungsinstrument diesen handlungstheoretischen Dreiklang (Leistungsfähigkeit, Wille, Gelegenheiten) abbilden. Das steigert die Komplexität und erfordert - im Kontext einer Befragung - von der befragten Person kognitive Kompetenzen, die nicht immer vorausgesetzt werden können.

Insgesamt geht es bei der Diskussion dieser beiden Zugänge nicht darum, den einen gegen den anderen auszuspielen. Beide Zugänge setzen unterschiedliche Akzentuierungen und ergänzen sich, sodass die Wahl des Zugangs - wie eigentlich immer - von der Zielsetzung der Erhebung, der Zielpopulation und Stichprobe sowie vom Verwendungszweck abhängt.

Zu diskutieren, wie Barrierefreiheit im Rahmen standardisierter Befragung umsetzbar erscheint, ist method-driven, also eine Diskussion, die von der Methode ausgeht. Vorgelagert wäre eigentlich die Frage zu beantworten, ob nicht andere Erhebungsmethoden geeigneter wären, Barrieren und Barrierefreiheit zu erfassen. Einen Kandidaten dafür stellt die Beobachtung dar (vgl. Häder 2010, 299ff.), die als nicht-sprachbasierte Erhebungsmethode gerade in Bezug auf Menschen mit kognitiv-kommunikativen Beeinträchtigungen prädestiniert erscheint. In Form einer nicht-teilnehmenden Beobachtung könnten Menschen mit verschiedenen Beeinträchtigungen in Person- 
Umwelt-Interaktionsprozessen systematisch beobachtet werden: Wie gelingt das Einkaufen, das Essen-Gehen in einem Restaurant, der Besuch einer Arztpraxis? Welche Hindernisse der gestalteten Umwelt wirken wie auf die Aktivitäten und Teilhabemöglichkeiten? Denkbar wäre, hier auch digitale Forschungsmethoden - etwa virtuelle Feldexperimente - zum Einsatz zu bringen und das Erhebungsdesign als Selbstbeobachtung (vgl. ebd., 304) anzulegen, indem den Teilnehmenden bestimmte Situationen symbolisiert und standardisiert dokumentiert werden.

Zur Annäherung an das Konstrukt der Barrierefreiheit bei Personen mit kognitiven Beeinträchtigungen plädiert Trescher $(2018,163)$ für den „Rückgriff auf eine offenere Interviewgestaltung " als dies im Rahmen standardisierter Erhebungen möglich ist. So würden sich erlebte Barrieren bei dieser Personengruppe oftmals nicht in einer Ad-hoc-Befragungssituation, sondern erst bei vertiefenden Thematisierungen im Rahmen offener Verfahren zeigen. Um dieses Resümee aufzugreifen, aber dennoch in einem standardisierten Rahmen zu bleiben, wäre eine Einbindung von offenen Methoden in Form eines Mixed-Methods-Designs möglich, in dem etwa bei einem identischen Sample sowohl qualitative als auch quantitative Daten erhoben und die qualitativen Forschungsresultate anschließend in quantitative Daten transformiert werden (vgl. Kuckartz 2018, 172).

Ungeachtet der Wahl der Erhebungsmethode und des Zugangs bleibt eine Messung von Barrieren komplex. Barrieren und Barrierefreiheit bilden relationale Gefüge, die sich als Verhältnis von Umweltbedingungen, individuellen Komponenten und Aktivitäten immer wieder neu formieren. Nur so erklärt sich, warum Barrieren in der empirischen Forschung noch wenig im Blickfeld stehen. An der politischen und lebenspraktischen Bedeutung kann es zweifellos nicht liegen: Eine barrierefreie Umwelt stellt für Menschen mit Beeinträchtigungen eine Voraussetzung dar, um in vielen gesellschaftlichen Lebensbereichen teilzuhaben.

\section{Literatur}

Aktion Mensch (2017): Faktenblatt Barriere-Barometer 2017, München. Online unter: https://www. aktion-mensch.de/dam/jcr:724b5f20-d3ea-40f8-b302-11bde951b76d/aktion-mensch-faktenblatt-muenchen.pdf. (Abrufdatum: 02.12.2019).

BMAS (Bundesministerium für Arbeit und Soziales) (2017): Teilhabebericht der Bundesregierung über die Lebenslagen von Menschen mit Beeinträchtigungen 2016, Bundestags-Drucksache 18/10940 vom 20.01.2017.

Bortz, J. \& Döring, N. (2006): Forschungsmethoden und Evaluation für Human- und Sozialwissenschaftler. 4. Aufl. Heidelberg: Springer.

Deutsches Studentenwerk (Hrsg.) (2012): Beeinträchtigt studieren. Online unter: https://www.studentenwerke.de/sites/default/files/Beeintraechtigt_Studieren_Datenerhebung_01062012_0.pdf. (Abrufdatum: 02.12.2019).

Diekmann, A. (2004): Empirische Sozialforschung. 11. Aufl. Reinbek: Rowohlt.

Faulbaum, F., Prüfer, P. \& Rexroth, M. (2009): Was ist eine gute Frage? Die systematische Evaluation der Fragenqualität. Wiesbaden: VS. 
Häder, M. (2010): Empirische Sozialforschung. Eine Einführung. 2. Aufl. Wiesbaden: VS.

Kastl, J.M. (2017): Einführung in die Soziologie der Behinderung. 2. Aufl. Wiesbaden: VS.

Kuckartz, U. (2018): Datenanalyse in der Mixed-Methods-Forschung. Strategien der Integration von qualitativen und quantitativen Daten und Ergebnissen. In: N. Baur, U. Kelle \& U. Kuckartz (Hrsg.): Mixed Methods. Sonderheft der Kölner Zeitschrift für Soziologie und Sozialpsychologie 69 (2), 157-183.

Nordenfelt, L. (2003): Action theory, disability and ICF. In: Disability \& Rehabilitation 25(18), 10751079.

Rambausek, T. (2017): Behinderte Rechtsmobilisierung. Wiesbaden: VS.

Schäfers, M., Schachler, V., Schneekloth, U., Wacker, E. \& Zeiler, E. (2016a). Pretest Befragung in Einrichtungen der Behindertenhilfe im Auftrag des Bundesministeriums für Arbeit und Soziales (BMAS). Online unter: http://www.bmas.de/SharedDocs/Downloads/DE/PDF-Publikationen/ Forschungsberichte/fb471-pretest-befragung-in-einrichtungen.pdf. (Abrufdatum: 02.12.2019).

Schäfers, M., Schachler, V., Schneekloth, U., Wacker, E. \& Zeiler, E. (2016b). Anhang C1: Endversion: Instrument Selbstauskunft. Online unter: https://www.bmas.de/SharedDocs/Downloads/DE/ PDF-Publikationen/Forschungsberichte/fb471-anhang-c1.pdf. (Abrufdatum: 02.12.2019).

Schnell, R., Hill, P.B. \& Esser, E. (2013): Methoden der empirischen Sozialforschung. 10. Aufl. München: Oldenbourg Verlag.

Schröder, H., Steinwede J., Schäfers, M., Kersting, A. \& Harand, J. (2017): Repräsentativbefragung zur Teilhabe von Menschen mit Behinderungen. 1. Zwischenbericht. Online unter: http://www. bmas.de/DE/Service/Medien/Publikationen/Forschungsberichte/Forschungsberichte-Teilhabe/fb492-repraesentativbefragung-behinderung.html. (Abrufdatum: 02.12.2019).

Schröttle, M. \& Hornberg, C. (2014a): Vorstudie für eine Repräsentativbefragung zur Teilhabe von Menschen mit Behinderung(en). Abschlussbericht. Online unter: https://pub.uni-bielefeld.de/ download/2727525/2727560/forschungsbericht-vorstudie-repraesentativbefragung-zur-teilhabevon-menschen-mit-behinderung.pdf. (Abrufdatum: 02.12.2019).

Schröttle, M. \& Hornberg, C. (2014b): Vorstudie für eine Repräsentativbefragung zur Teilhabe von Menschen mit Behinderung(en). Anhang A: Quantitativer Fragebogen. Unveröffentl. Manuskript.

Schuntermann, M.F. (2004): Einführung in die Internationale Klassifikation der Funktionsfähigkeit, Behinderung und Gesundheit (ICF) der Weltgesundheitsorganisation (WHO) unter besonderer Berücksichtigung der sozialmedizinischen Begutachtung und Rehabilitation. Online unter: http://www. pulsmesser.ch/wp-content/uploads/2010/11/ICF-Grundkurs.pdf. (Abrufdatum: 02.12.2019).

Trescher, H. (2018): Kognitive Beeinträchtigung und Barrierefreiheit. Eine Pilotstudie. Bad Heilbrunn: Klinkhardt.

WHO (World Health Organization) (2001): International Classification of Functioning, Disability and Health. Geneva: WHO.

WHO (World Health Organization) \& The World Bank (o.J.): Model Disability Survey. Brief Version. Online unter: https://www.who.int/disabilities/data/brief-model-disability-survey5.pdf. (Abrufdatum: 02.12.2019). 



\section{Anwendungsfelder}

\section{Matthias Schmidt-Ohlemann \\ Barrierefreie Gesundheitsversorgung - zwischen Zugänglichkeit allgemeiner Versorgung und Notwendigkeit besonderer Einrichtungen}

\section{Einleitung}

Nach Art. 1 der UN-Behindertenrechtskonvention (UN-BRK) ist es Zweck dieser Konvention, „die volle und gleichberechtigte Ausübung aller Menschenrechte und Grundfreiheiten durch alle behinderten Menschen zu fördern, zu schützen und zu gewährleisten und die Achtung ihrer angeborenen Würde zu fördern.“ Zweifellos gehört das Gesundheitswesen zu den Bereichen der Gesellschaft, zu denen Menschen mit Behinderungen gleichberechtigt, uneingeschränkt und in vollem Umfang Zugang haben sollen.

Barrierefreiheit im Gesundheitswesen im Sinne der UN-BRK (Art. 25 und 26) bedeutet die Gleichbehandlung aller Menschen, die Sicherstellung eines gleichberechtigten Zugangs zum Gesundheitswesen, die Barrierefreiheit aller Dienstleistungen des Gesundheitswesens und die Vermeidung von Diskriminierung. Dazu gehören nicht nur räumliche und sachliche Umweltfaktoren, sondern auch soziale und gesellschaftliche, z.B. Einstellungen, Umgangsweisen mit Krankheit und Behinderung. (BAR 2012; Welti 2016)

Als Barriere gilt auch das Fehlen von Förderfaktoren (Kontextfaktoren im Sinne der ICF) bzw. das Fehlen angemessener Vorkehrungen. Zu prüfen ist deshalb, ob solche im Gesundheitswesen getroffen worden sind, und zwar als Bereitstellung von besonderen Gesundheitsleistungen, die „von Menschen mit Behinderungen speziell wegen ihrer Behinderungen benötigt werden..." (Art. 25 UN-BRK) und von Leistungen der Rehabilitation, hier der medizinischen Rehabilitation, als medizinische Intervention zur Verbesserung der Möglichkeiten für umfassende und selbstbestimmte Teilhabe aller Bürgerinnen und Bürger (Art. 26 UN-BRK). Denn 
auch Beeinträchtigungen von individuellen Kompetenzen, die die Nutzung des Gesundheitssystems erschweren oder einer selbstbestimmten Teilhabe entgegenstehen, können Barrieren darstellen, wobei diesen durch Leistungen der Rehabilitation im Sinne der Kompetenzerweiterung oder durch Schaffung von Förderfaktoren entgegengewirkt werden kann.

Insofern erweist sich eine Bestimmung von Barrierefreiheit im Gesundheitswesen, die nur auf den Zugang zu vorhandenen Gesundheitsleistungen zielt, als zu eng. Denn es geht um die Sicherstellung einer bedarfsgerechten Gesundheitsversorgung (Diagnostik, Therapie und Rehabilitation) im Sinne angemessener staatlicher Vorkehrungen, d.h. auch um das bedarfsgerechte Vorhalten entsprechender Dienste und Einrichtungen und die Gewährleistung ihrer Inanspruchnahme durch die rechtliche Absicherung der Ansprüche.

Der Begriff behinderte Menschen umfasst nach Art. 1 Abs. 2 UN-BRK „Menschen mit langfristigen körperlichen, seelischen, geistigen oder Sinnesschädigungen, die sie im Zusammenwirken mit verschiedenen Barrieren daran hindern können, gleichberechtigt mit anderen uneingeschränkt und wirksam an der Gesellschaft teilzunehmen." Dem folgt $\$ 2$ SGB IX.

Die Gruppe der Menschen mit Behinderung ist dabei heterogen: Denn die den Behinderungen zugrundeliegenden Gesundheitsstörungen sind sehr unterschiedlich und betreffen ganz unterschiedliche Körperfunktionen und Aktivitäten. Für Menschen mit körperlichen, intellektuellen, seelischen oder Sinnesbehinderungen sind ganz verschiedene Barrieren relevant. Diese Breite gilt es stets zu beachten. Zusätzlich sind weitere Krankheiten von Menschen mit Behinderung zu berücksichtigen, die diese mit weiteren Barrieren konfrontieren können. (Degener u.a. 2015)

\section{Die Angebote des Gesundheitswesens}

Das Gesundheitswesen muss in der Lage sein, die gesundheitliche Versorgung entsprechend dem Bedarf der Menschen mit Behinderungen für diese im gleichen Umfang zu übernehmen wie für Menschen ohne Behinderungen. Dabei kann man schematisch drei Formen der gesundheitlichen Versorgung (jeweils ambulant, mobil oder stationär) unterscheiden (vgl. Schmidt-Ohlemann 2017):

1. Allgemeine und fachärztliche Diagnostik und Behandlung bei allgemeinen Krankheiten im Rahmen der Regelversorgung (leistungsrechtlich Krankenbehandlung der Regelversorgung im Rahmen des SGB V).

2. spezialisierte Diagnostik und Behandlung bei sehr speziellen oder besonderen, oft mit Behinderungen assoziierten Krankheiten, Krankheitsausprägungen und Syndromen bzw. Syndromkombinationen: hoch spezialisierte Versorgung z.B. in Spezialambulanzen, z.B. der Hochschulen oder in anderer Trägerschaft, oder durch besondere Einrichtungen (leistungsrechtlich Krankenbehandlung im Rahmen des $S G B V$ ). 
3. Assessment-, Behandlungs- und/oder medizinische Rehabilitationsleistungen zur Verminderung von Auswirkungen von körperlichen, geistigen, seelischen oder Sinnesbeeinträchigungen auf die Teilhabe (vgl. zu den Zielen insbesondere $\$ 42$ SGB IX; leistungsrechtlich Leistungen zur medizinischen Rehabilitation oder rehabilitationsmedizinische Versorgung in anderen Settings im Rabmen des SGB IX in Verbindung mit trägerspezifischen Leistungsgesetzen, z.B. SGB V, VI, VII und VIII).

Besondere Einrichtungen der Gesundheitsversorgung für Menschen mit (drohender) Behinderung zeigt Tabelle 1 .

Tab. 1: Besondere Einrichtungen der Gesundheitsversorgung für Menschen mit (drohender) Behinderung

- Sozialpädiatrische Zentren (SPZ, \$\$ 43a und 119 SGB V)

- Frühförderstellen zur Erbringung von interdisziplinärer Frühförderung (\$ 46 SGB IX)

- Medizinische Behandlungszentren für Erwachsene Menschen mit geistiger Behinderung oder schweren Mehrfachbehinderungen (MZEB, $\$ \$ 43 \mathrm{~b}$ und 119c SGB V)

- Psychiatrische Institutsambulanzen (PIA $\$ 118$ SGB V)

- Einrichtungen der ambulanten spezialfachärztlichen Versorgung (ASV $\$ 116 b$ SGB V)

- Hochschulambulanzen mit speziellen Versorgungsaufgaben (\$ 117 SGB V)

- Integrierte ärztliche und therapeutische Dienste in (Förder-)Kindertagesstätten, Schulen, Berufsbildungswerken (BBW)/Berufsförderungswerken (BFW) oder Wohneinrichtungen, die Leistungen zur Erziehung, Bildung oder beruflichen oder sozialen Teilhabe erbringen, soweit diese für die Erreichung der Ziele erforderlich sind (vgl. z.B. $\$ 10$ Werkstättenverordnung), z.B. auch Beratungsstellen für Unterstützte Kommunikation (zur leistungsrechtlichen Zuordnung vgl. Schmidt-Ohlemann 2017 und 2018)

- Fachkrankenhäuser für Menschen mit geistigen oder mehrfachen Behinderungen, Querschnittlähmung o.ä.

- Spezialisierte Einrichtungen der medizinischen Rehabilitation, z.B. Rehabilitationseinrichtungen für psychisch Kranke (RPK), oder mit Aufnahmemöglichkeit bei besonderen Indikationen, z.B. Sehbehinderung, oder der Mobilen Rehabilitation für mobilitätsbeeinträchtigte Menschen mit ggf. weiteren relevanten Teilhabebeeinträchtigungen

Solche Einrichtungen weisen eine besondere Spezialisierung auf bestimmte Krankheitsgruppen auf, bei denen Art und Schwere der Erkrankungen sowie der möglichen Beeinträchtigungen der Teilhabe besondere Angebote notwendig machen. Sie werden von Menschen mit (drohender) Behinderung speziell wegen ihrer Behinderung benötigt, sei es auf Grund der die Behinderung bedingenden, oft seltenen Erkrankung, sei es auf Grund spezifischer, umfassender oder schwerwiegender Co- oder Multimorbidität, die besondere Kenntnisse, Erfahrungen, Settings und Ressourcen erfordern, die im allgemeinen Gesundheitssystem nicht oder nicht ausreichend vorhanden sind. (vgl. dazu den Tagungsband Seidel 2015; Schmidt-Ohlemann 2014) 


\section{Mögliche Leistungen für Gesundheit und bei Krankheit}

Das Gesundheitswesen sieht eine Fülle von verschiedenen Leistungen vor, die durch unterschiedliche Leistungsträger erbracht werden. $\mathrm{Zu}$ den wesentlichen Leistungsträgern siehe Tabelle 2 .

Tab. 2: Sozialleistungsträger im Bereich der Gesundheitsversorgung

A. Gesetzliche Krankenversicherung (GKV), insbesondere für

1. Ärztliche Leistungen, z.B. Hausärztliche Leistungen, Fachärztliche Leistungen, Psychotherapeutische Leistungen, Zahnärztliche Leistungen

2. Krankenhausleistungen

3. Veranlasste Leistungen (ambulant), z.B. Arznei- und Verbandmittel, Heilmittel, Hilfsmittel zur Krankenbehandlung, Häusliche Krankenpflege, Soziotherapie, Krankentransport

4. Leistungen der medizinischen Rehabilitation

B. Soziale Pflegeversicherung

C. Rentenversicherung (für Leistungen der medizinischen Rehabilitation)

D. Private Kranken-, ggf. Zusatz- und Pflegeversicherung sowie Beihilfe

E. Andere Träger, z.B. Unfallversicherung, Integrationsämter

F. Eingliederungshilfe, insbesondere für

1. Leistungen der medizinischen Rehabilitation bei Fehlen eines vorrangigen Leistungsträgers

2. Leistungen zur sozialen Teilhabe, die der Unterstützung der behinderten Menschen bei der Gesundheitssorge dienen, als Assistenzleistungen (vgl. $\$ 78$ Abs. 1 und $\$ 113 \mathrm{ff}$. SGB IX)

3. Heilpädagogische Leistungen

4. Leistungen zum Erwerb und Erhalt praktischer Kenntnisse und Fähigkeiten

5. Pflege, soweit sie die einfache Behandlungspflege nicht übersteigt

6. Hilfsmittel

7. Hilfen zur Mobilität

Hinzu kommen private Leistungen in erheblichem Umfang, die von Betroffenen für die Gesundheitssorge aufgewendet werden müssen. Dazu gehören z.B. persönliche Leistungen im Privathaushalt, wie $\mathrm{Zu}$ - und Aufzahlungen, persönliche Hygiene und Hautpflege, für nichtverschreibungspflichtige Arzneimittel, für Hilfsmittel von geringem Wert oder Gebrauchsgegenstände des täglichen Lebens, für Pflege, für Beförderung etc. (Finanzierung ggf. im Rahmen der Hilfe zum Lebensunterhalt bzw. Grundsicherung, ggf. einschließlich Mehrbedarfen). Wie umfangreich das Leistungsspektrum der Gesundheitsversorgung ist, wird am Beispiel der Gesetzlichen Krankenversicherung (GKV) in Tabelle 3 verdeutlicht. 
Tab. 3: Leistungsspektrum der Gesetzlichen Krankenversicherung (GKV)

\section{A. GKV Leistungen nach $\$ \$ 11,27$ SGB V u.a.}

A.1. Leistungen der ambulanten Krankenbehandlung und Prävention, z.B.

A.1.1. Hausärztliche Leistungen

A.1.2. Fachärztliche Leistungen

A.1.3. Psychotherapeutische Leistungen

A.1.4. Zahnärztliche Leistungen

A.1.5. Besondere Formen (vgl. auch Tab. 1)

1. Vorsorge/Früherkennungsuntersuchungen $(\$ \$ 20 \mathrm{ff}$. SGB V) für Kinder und Jugendliche sowie Erwachsene

2. Disease-Management-Programme (DMP)

3. Leistungen zur Prävention ( $\$ \$ 20 \mathrm{ff}$. SGB V)

4. Psychiatrische Institutsambulanzen (PIA), Sozialpädiatrische Zentren (SPZ), Medizinische Behandlungszentren für erwachsene Menschen mit Behinderung (MZEB), heimärztliche Versorgung (Pflegeheime $\$ 119 \mathrm{~b}$ SGB V), ambulante spezialfachärztliche Versorgung, Hochschulambulanzen

A.1.6. Arznei- und Verbandmittel

A.1.7. Heilmittel, Hilfsmittel

A.1.8. Häusliche Krankenpflege, Kurzzeitpflege (\$39c SGB V)

A.1.9. Soziotherapie

A.1.10. Krankentransporte

A.1.11. Haushaltshilfe

A.1.12. Nichtärztliche Leistungen ( $\$ 43$ a SGB V), MZEB ( $\$ 43$ b SGB V), sozialmedizinische Nachsorge für Kinder und Jugendliche ( $\$ 43$ Abs. 2 SGB V)

A.1.13. spezialisierte ambulante Palliativversorgung (SAPV)

A.2. Krankenhausleistungen einschließlich Frührehabilitation

A.3. Medizinische Rehabilitation und med. Vorsorge sowie ergänzende Leistungen zur Rehabilitation

A.4. Weitere Leistungen durch Vertragsärzte, z.B.

A.4.1. Anordnung der Hilfeleistungen anderer Personen (\$73 Abs. 2 Nr. 6 SGB V)

A.4.2. Bescheinigungen und Berichte für Krankenkasse bzw. Medizinischen Dienst der Krankenversicherung $(\mathrm{MdK})$ und andere Sozialleistungsträger (\$73 Abs. 2 Nr. 9 SGB V)

All diese Leistungen wie auch die der anderen zuständigen Leistungsträger, z.B. für die medizinische Rehabilitation, siehe Tabelle 2, stehen grundsätzlich auch Menschen mit Behinderung zur Verfügung. Zugangsbarrieren können sich neben settingbezogenen Barrieren (vgl. Tabelle 4) auch durch fehlende Verfügbarkeit oder Erreichbarkeit von Einrichtungen und Diensten ergeben. So sind z.B. MZEB 
oder mobile Rehabilitationsdienste nicht flächendeckend verfügbar. Auch gibt es zahlreiche, Art und Umfang der Leistungen begrenzende Bestimmungen wie etwa die Richtlinien des Gemeinsamen Bundesausschusses, die z.B. den Anspruch auf Beförderung auf einige Personengruppen begrenzen, oder die Gesamtverträge nach $₫ 83$ SGB V, die Leistungsvereinbarungen zu Heil- und Hilfsmitteln sowie zahlreiche weitere Verträge, bei denen Belange behinderter Menschen nicht oder unzureichend berücksichtigt werden.

Bei den Aufnahmekriterien der Einrichtungen der medizinischen Rehabilitation wird in der Regel vorausgesetzt (Ausnahmen sind spezifische Indikationsbereiche wie die Geriatrie oder Neurologie), dass kein erheblicher Pflegebedarf besteht und eine eigenständige Mobilität auf der Stationsebene gegeben ist. Auch wird bei Menschen mit Behinderung auf Grund der vorliegenden Beeinträchtigungen oft ein Rehabilitationspotential verneint. Dies führt dazu, dass Menschen mit Behinderung und Assistenzbedarf oder Pflegebedürftigkeit faktisch von der Inanspruchnahme der meisten stationären und ambulanten Einrichtungen zur indikationsspezifischen medizinischen Rehabilitation ausgeschlossen sind. ${ }^{1}$

\section{Krankenbehandlung bei Menschen mit Behinderung}

Menschen mit Behinderungen können krank werden, d.h. alle Krankheiten bekommen, die alle anderen Menschen auch bekommen können. Ferner liegt der Behinderung definitionsgemäß immer eine Gesundheitsstörung zugrunde, die sich nach der ICD 10 kodieren lässt, und die ggf. einer Behandlung bedarf. Dennoch gilt: Behinderung als solche ist keine Krankheit!

Die Gruppe der Menschen mit Behinderung, auch mit intellektueller oder seelischer Behinderung weist aber eine höhere allgemeine und zudem oft eine spezifische (Co-)Morbidität auf. Sie müssen im regulären Versorgungssystem sachgerecht behandelt werden. Leistungen der Gesundheitsversorgung sind oft auch notwendig, um die Beeinträchtigungen auf der Funktionsebene selbst zu vermindern oder zu beseitigen, dienen also nicht nur der „Krankheitsbehandlung“, sondern zumindest mittelbar auch der Verbesserung der Voraussetzungen für eine selbstbestimmte Teilhabe. Die gesetzliche Grundlage dafür besteht in $\$ 43$ SGB IX. Deren bedarfsgerechte Gesundheitsversorgung wird oft durch Barrieren behindert: Diese können im baulichen und technischen Umfeld liegen, wenn die vorhandenen technischen Standards zur Barrierefreiheit nicht berücksichtigt werden, im sozialen Umfeld (Einstellungen, fehlende Kompetenzen im Umgang mit Behinderung, in mangelnden Ressourcen bei der Sicherung der Inanspruchnahme oder auch in individuellen, personbezogene Faktoren (Kontextfaktoren der ICF),

1 Zum Thema Rehabilitation vor und bei Pflegebedürftigkeit erscheint im November 2020 ein umfassendes Positionspapier der DVfR. 
z.B. auf Grund von früheren Traumatisierungen durch medizinische Interventionen. Tabelle 4 zeigt einige Beispiele für typische Barrieren auf:

Tab. 4: Beispiele für typische Barrieren für Menschen mit Behinderungen im Gesundheitswesen

- Bauliche Barrieren (Praxen von Ärzten, Therapeuten): Treppen, WC

- Fehlende Hilfsmittel (z.B. Lifter, Liegen/Untersuchungsstühle)

- Komplizierte Sprache - Fehlen einfacher Sprache

- Ignorierung der betroffenen Person und Kommunikation nur mit der Begleitperson

- Unverständnis und Ungeduld bei Ängsten, Ärger bei mangelnder Kooperation

- Unzureichende Zeitbudgets bei der Konsultation, allgemeiner Zeitdruck und Überlastung

- Fehlende Kenntnis von spezifischen Krankheitsbildern und Syndromkombinationen

- Mangelnde Kompetenzen bei der Symptomerkennung, v.a. bei fehlender Sprache und bei der Interpretation von Symptomen, die oft atypisch präsentiert werden

- Fehlen von Gebärdendolmetschern (ggf. auch Übersetzer von Fremdsprachen)

- Fehlen von barrierefreien Unterlagen für Menschen mit Sehbehinderung

- Fehlende Unterstützung während medizinischer Maßnahmen, insbesondere im Krankenhaus

- Fehlende Angebote in Rehaeinrichtungen für Menschen mit intellektuellen oder mehrfachen Beeinträchtigungen oder Pflegebedürftigkeit (Ausschluss wegen mangelnder Rehafähigkeit)

- Mangel an mobilen Angeboten für immobile Patienten

- Fehlendes Fallmanagement zur Bewältigung der Barrieren bei der Inanspruchnahme von gesundheitsbezogenen Leistungen im gegliederten System der Sozialleistungen

Zur Barrierefreiheit im Gesundheitswesen existieren kaum belastbare Daten (vgl. dazu insbesondere Welti 2016). Immerhin wird der Aussage, dass ca. zwei Drittel der vorhandenen Arztpraxen derzeit nicht barrierefrei sind, nicht widersprochen. ${ }^{2}$

\section{Barrierefreiheit als gesamtgesellschaftliche Strategie der Teilhabesicherung, nicht nur in der Gesundheitsversorgung}

Dass mannigfache Barrieren im Gesundheitswesen für Menschen mit Behinderungen, und zwar im Bereich sowohl der Krankenbehandlung als auch der Rehabilitation bestehen, dürfte unstrittig sein. Vernachlässigt wird dabei oft, dass auch für Menschen, die dem Personenkreis der behinderten Menschen in einer alltäglichen Betrachtungsweise nicht zugerechnet werden, zahlreiche Barrieren bestehen können, z.B. für geriatrische Patienten, chronisch Kranke etc. oder auch durch Fremdsprachlichkeit oder Armut.

2 Auf eine quantifizierende Darstellung wird deshalb hier zugunsten einer systematischen Betrachtungsweise verzichtet. 
Strategien zur Beseitigung von Barrieren sollten deshalb allgemein und nicht nur auf die Gruppe von Menschen mit Behinderungen, die z.B. Anspruch auf Leistungen der Eingliederungshilfe hat oder Unterstützungen im Bereich der Behindertenhilfe erhält, ausgerichtet sein. Solche Strategien sind z.B.: Umsetzung eines Universal Design: z.B. bei Bauten, im ÖPNV, bei Zu- und Aufzahlungen, Bereitstellung von Fallberatung, -begleitung und -management, barrierefreie IT- und Kommunikationssysteme etc. (vgl. dazu BAR 2012).

Die Beseitigung der vielfachen Barrieren für Menschen mit Behinderungen erfordert zahlreiche einzelne Maßnahmen sowie einen Bewusstseinswandel. Hier gibt es zahlreiche meist nur punktuelle Ansätze und Bemühungen. ( $\mathrm{Zu}$ den rechtlichen Grundlagen und zu ihrer Umsetzung vgl. Welti 2016). Dies gelingt jedoch nur partiell. Dies liegt auch daran, dass die Herstellung von Barrierefreiheit nicht nur ein Umdenken, sondern vielfach auch die Bereitstellung von zusätzlichen Ressourcen bedeutet. Es sind dazu an vielen Stellen Entscheidungen im Hinblick auf eine entsprechende Mittelallokation erforderlich. Diese sind oft nicht unmittelbar aus den gesetzlichen Grundlagen, z.B. dem Allgemeinen Gleichbehandlungsgesetz (AGG) oder der UN-BRK ableitbar. Sie müssen vielmehr auch im fachlichen und v.a. auch im politischen Diskurs jeweils legitimiert und im jeweiligen Handlungskontext konkretisiert werden.

Teilhabeforschung sollte zur Legitimation von Forderungen nach Barrierefreiheit beitragen, u.a. durch:

- Beschreibung der Folgen von Barrieren, insbes. aus der Perspektive der Betroffenen,

- empirische Grundlagen für Priorisierungsentscheidungen,

- Evaluation von Maßnahmen zur Verminderung von Barrieren,

- Identifikation von Gesundheitsleistungen, die Beeinträchtigungen vermindern.

Forschungsergebnisse können somit zur Herstellung von Barrierefreiheit beitragen.

\section{Besondere Einrichtungen der Gesundheitsversorgung - eine Folge mangelnder Barrierefreiheit?}

Führt nun die mangelnde Barrierefreiheit im Gesundheitswesen zur Errichtung der besonderen Einrichtungen für Menschen mit (drohenden) Behinderungen? Dies ist im Hinblick auf die in Tabelle 1 benannten Einrichtungen zu verneinen. Sie sind vielmehr überwiegend Ausdruck einer differenzierten Arbeitsteilung innerhalb des Gesundheitswesens, die eine Bedarfsdeckung ermöglichen soll, wenn besondere Bedarfe bestehen. Denn es handelt sich hier um Einrichtungen und Dienste, die besondere Bedarfe befriedigen, denen im allgemeinen Gesundheitswesen mit den dort vorhandenen Kompetenzen und wegen der fehlenden spezifisch erforderlichen Interdisziplinarität sowie spezifischer Settings nicht ent- 
sprochen werden kann. Ferner können auf Grund der dort vorherrschenden Leistungs- und Vergütungssystematik notwendige Angebote nicht bzw. nur schwer organisiert werden. (vgl. Schmidt-Ohlemann 2014 am Beispiel der MZEB)

Unabhängig von besonderen Bedarfen bleiben Menschen mit Behinderung auf die Leistungen des allgemeinen Gesundheitswesens angewiesen und nutzen diese auch vorrangig. So kann z.B. auf die haus- und fachärztliche ambulante Versorgung, auf Krankenhausbehandlung bei akuten Erkrankungen, auf die reguläre Heil- und Hilfsmittelversorgung nicht verzichtet werden. Vielmehr ist von einem gestuften Versorgungskonzept auszugehen, bei dem die besonderen Einrichtungen und Dienste nur dann ergänzend genutzt werden, wenn das Regelversorgungssystem mit der Behandlung überfordert ist. (Einen guten Überblick über Versorgungsfragen behinderter Menschen gibt der Tagungsband von Seidel 2015 mit zahlreichen Einzelbeiträgen.)

Dabei muss dennoch erreicht werden, dass das allgemeine Gesundheitssystem durch Barrierefreiheit und erweiterte Ressourcen in die Lage versetzt wird, Menschen mit Behinderung angemessen und umfassend zu behandeln. Dies findet aber eine Grenze in der nicht überall verfügbaren, aber notwendigen Spezialisierung zur Bewältigung der besonderen Bedarfe und der Problemlagen von Menschen mit Behinderungen. Würde man diesen eine spezialisierte Behandlung in besonderen Einrichtungen vorenthalten, würde das einer Diskriminierung gleichkommen: Denn jede Bürgerin und jeder Bürger wünscht sich eine fachlich kompetente Behandlung durch Spezialist*innen, wenn dies erforderlich ist: Wer würde eine allgemeininternistische einer spezialisierten onkologischen Behandlung eines bösartigen Tumors vorziehen?

Dennoch ist nicht von der Hand zu weisen, dass besondere Einrichtungen auch deshalb gefordert und geschaffen werden, weil das allgemeine Gesundheitswesen nicht barrierefrei ist. Dies ist aber nicht der systematische und relevante Grund für die Bereitstellung besonderer Einrichtungen: Barrierefreiheit im Gesundheitswesen macht besondere Einrichtungen und Dienste nicht überflüssig.

\section{Besondere Bedarfe bei der Krankenbehandlung}

Art. 25 UN-BRK sieht dementsprechend auch vor, dass Menschen mit Behinderungen auch auf solche Angebote zugreifen können, die sie speziell wegen ihrer Behinderung benötigen. Daraus geht schon hervor, dass die Existenz besonderer Bedarfe grundsätzlich anerkannt wird. Offen bleibt, wie die Systeme der Gesundheitsversorgung diese besonderen Bedarfe berücksichtigen sollen.

Worin bestehen die besonderen Bedarfe? Hierzu einige Beispiele:

- Menschen mit (intellektuellen) Behinderungen leiden oft an sehr seltenen, häufig genetisch oder pränatal bedingten Gesundheitsstörungen bzw. Krankheiten. 
- Sie zeigen ungewöhnliche und unspezifische Symptome, auch bei schweren Erkrankungen.

- Sie leiden an unerwarteten oder schwierig zu erkennenden oder zu behandelnden Komplikationen.

- Für die Diagnostik und Behandlung müssen gut vertraute Bezugspersonen eng einbezogen werden.

- Die Diagnosestellung ist oft sehr schwierig und erfordert oft zusätzliche aufwändige technische Untersuchungen.

- Besondere Herausforderungen bietet die Kombination von geistiger Behinderung und psychischer Erkrankung.

- Behandlungsstrategien bei Menschen mit intellektuellen Behinderungen können sich von denen bei Menschen ohne Behinderungen unterscheiden und müssen oft individuell variiert werden. Zugleich aber funktionieren reguläre Therapien entgegen den vorurteilsbehafteten Erwartungen gerade doch, z.B. die Endoprothetik großer Gelenke.

- Prognostische Aussagen sowie die Einschätzung der Krankheitslast sind oft schwierig. Die Durchführung von Untersuchungen und Behandlungen gestaltet sich oft aufwändig und müssen sorgfältig geplant und im Hinblick auf den Nutzen besonders abgewogen werden. Therapeutische Strategien sollten sich auch auf die Verminderung der Beeinträchtigungen und damit das Behinderungsausmaß richten, ohne dass die zugrundeliegende Krankheit selbst behandelt werden kann, z.B. Mobilitätseinschränkungen bei Infantiler Cerebralparese (vgl. dazu Martin 2019; Sappok u.a. 2019).

Deshalb ist oft eine Art therapeutischer Nihilismus oder eine Übertherapie bei der Behandlung von Menschen mit Behinderung zu finden, v.a. wenn es an spezifischen Erfahrungen mangelt. Auf Grund von Fehlbehandlungen sind regelmäßig vermeidbare Komplikationen oder gar Todesfälle zu beobachten.

Häufige Krankheiten oder Syndrome, die zudem oft in Kombinationen auftreten und in Kombination mit schwerer intellektueller, körperlicher, seelischer oder Sinnesbehinderung besondere diagnostische und therapeutische Strategien erfordern, zeigt Tabelle 5 .

Tab. 5: Häufige (Begleit-)Diagnosen und Symptome bei Menschen mit Behinderungen

- Epilepsie

- Unklare Schmerzzustände

- Gastrooesophagealer Reflux

- Obstipation und Koprostase

- Inkontinenz 
- Decubitus, schlecht heilende Wunden

- Atypische Bewegungsmuster/Aufgabe vorhandener Bewegungskompetenzen

- Lähmungen/Spastik

- Fehlende Sprache

- Schwere körperliche Bewegungseinschränkungen

- Sinnesbeeinträchtigungen (Sehen, Hören)

- Kombination von intellektuellen und seelischen Behinderungen mit atypischer Symptomausprägung

- Paradoxe Reaktionen

- Herausforderndes Verhalten

Die Behandlung gestaltet sich zusätzlich schwierig, wenn es sich um seltene Erkrankungen oder atypische Symptome und Symptomkombinationen handelt. Meist besteht zudem ein hoher psychosozialer Beratungsbedarf bei den Betroffenen und ihren Bezugspersonen. Bei Mehrfachbehinderungen ist oft eine umfangeiche Hilfsmittelversorgung notwendig, die nur in einem interdisziplinären Team aus Rehabilitationsmittelfachkräften, Therapeuten, Pflegekräften, pädagogischen Bezugspersonen und den behandelnden Ärzten erfolgreich sein kann. Hinzu kommt oft das Erfordernis umfassender sozialmedizinischer Beurteilung wegen notwendiger Sozialleistungen. Allein die Organisation solcher Versorgungsprozesse erfordert meist einen besonderen organisatorischen Aufwand (Terminvereinbarung, hoher Zeitbedarf, Beförderungsfragen, Bewältigung von Wartezeiten u.a. mit intermittierend notwendiger Pflege, Koordination der Zusammenarbeit).

Die fachlichen und die organisatorischen Anforderungen an die Behandlung von Menschen mit Behinderungen machen deshalb besondere Angebote durch Dienste und Einrichtungen mit Experten mit besonderer Kompetenz im Bereich Medizin für Menschen mit Behinderungen erforderlich. Diese sieht das System der Gesundheitsversorgung in Deutschland vor, vgl. dazu Tabelle 1, wenngleich nicht im erforderlichen Umfang. (Seidel 2015)

\section{Folgerungen}

Die bisherigen Ausführungen lassen sich thesenhaft so zusammenfassen:

1. Alle Menschen müssen Zugang zum allgemeinen Gesundheitssystem und zum System der Rehabilitation haben.

2. Dazu sind zahlreiche Barrieren abzubauen. Dies kann durch bauliche, organisatorische und finanzielle Maßnahmen, durch Aus- und Fortbildung aber auch durch Haltungs- und Einstellungsänderungen sowie durch angemessene Kommunikationsstrategien geschehen. 
3. Jeder Mensch mit Behinderung benötigt vorwiegend Versorgung im Regelsystem der allgemeinen Gesundheitsversorgung (u.a. durch Hausärzte, Fachärzte, Krankenhäuser etc.).

4. Sofern spezielle Problemkonstellationen bestehen, die im regulären Gesundheitssystem nicht bearbeitet werden können, müssen Menschen mit Behinderungen das Recht haben, auf ihre Probleme spezialisierte Experten und ggf. eine spezialisierte Versorgungseinrichtung in Anspruch nehmen zu können.

5. Unterlässt man diese Bereitstellung im Sinne angemessener Vorkehrungen der UN-BRK stellt das Fehlen solcher Versorgungsangebote eine Barriere zur Teilhabe am Leben der Gesellschaft, bzw. eine Diskriminierung im Sinne der UN-BRK dar.

6. Spezialisierte Versorgung wird auch gefordert und durchgeführt, da das reguläre Gesundheitssystem noch zu viele Barrieren aufweist: Das ist auf Dauer keine UN-BRK-konforme und eine weder politisch noch ethisch akzeptable Lösung! Hier muss der Abbau der Barrieren im Vordergrund stehen!

\section{Mangelnde Unterstützung bei der Gesundheitssorge von Menschen mit Behinderung als wesentliche Barriere für die Gesundheitsversorgung}

Neben den Struktur- und Prozesselementen des Gesundheitssystems darf jedoch die zentrale Rolle der Bürgerinnen und Bürger für die eigene Gesundheitsversorgung nicht unbeachtet bleiben. Aufgabe aller Bürger*innen (und Versicherten) ist es, soviel wie möglich selbst für die persönliche Gesundheit zu tun, und soweit sie das möchten, selbst die Einrichtungen und Dienste des Gesundheitswesens in Anspruch zu nehmen und diese Inanspruchnahme zu organisieren sowie die Anweisungen oder Empfehlungen der Ärzt*innen und Therapeut*innen umzusetzen. Um diesen Bereich der persönlichen Lebensführung zu beschreiben, wurde das Konzept der Gesundheitssorge entwickelt. (Schmidt-Ohlemann 2017; DVfR 2018 b)

Unter Gesundheitssorge soll all das verstanden werden, was Bürger*innen selbst zur Erhaltung ihrer Gesundheit und zur Bewältigung und Behandlung von Erkrankungen beizutragen haben. Dabei sind sie, je individuell unterschiedlich, auf Unterstützung ihres sozialen Umfeldes angewiesen und nehmen diese als selbstverständlichen Bestandteil ihres Lebens in Anspruch. Zur Gesundheitssorge im umfassenden Sinne gehört also auch die Begleitung und Unterstützung durch die Familie, z.B. bei Arztbesuchen, bei Krankenhausaufenthalt. Zur Gesundheitssorge gehören hingegen nicht die professionellen Leistungen im Rahmen der Krankenbehandlung, der Pflege, der medizinischen Reha oder der Prävention. Zur Gesundheitssorge gehören u.a. 
- eine gesunde Lebensführung,

- das rechtzeitige Bemerken von Krankheitsanzeichen,

- ein krankheitsadäquates Verhalten (Bettruhe) einschließlich Sicherstellung der Versorgung ggf. auch im Krankenhaus, soweit diese nicht durch das Krankenhaus erfolgt,

- die Selbstversorgung im Hinblick auf Pflege (bis hin zur Behandlungspflege, soweit diese leistbar ist),

- die bedarfsgerechte Inanspruchnahme von Leistungen der Gesundheitsversorgung (Termine bei Ärzt*innen, Therapeut*innen),

- die Befolgung ärztlicher, therapeutischer und pflegerischer Anordnungen (z.B. Besorgung und Einnahme von Medikamenten, Durchführung von Eigenübungen, die Anwendung von Hilfsmitteln),

- die Antragstellung auf Leistungen und Erledigung damit zusammenhängender organisatorischer Aufgaben (Transportgenehmigung),

- die Bereitstellung entsprechender finanzieller Mittel (Eigenanteile, Zuzahlungen etc.),

- die Sicherstellung der Beförderung zu Einrichtungen und Diensten des Gesundheitswesens,

- Organisation der Begleitung und Unterstützung bei Krankenhausaufenthalten.

Menschen mit Behinderung sind je nach deren Art und Schwere nicht immer in der Lage, die Gesundheitssorge im Rahmen ihres Alltagslebens selbstständig zu leisten. Sie verfügen zudem häufig nicht oder nicht ausreichend über ein primäres soziales Netz, das in der Lage ist, die Gesundheitssorge sicherzustellen. Sie haben auch meist nicht ausreichend Ressourcen, um sich selbst die notwendige Unterstützung zu verschaffen. Das Armutsrisiko ist stark erhöht. Insofern benötigen sie anderweitige Unterstützung bei der Gesundheitssorge.

Das Gesundheitswesen stellt nur einige Unterstützungsmaßnahmen für in der selbstständigen Gesundheitssorge gehinderten Menschen als Leistungen zur Verfügung, die allerdings nicht in jedem Setting gewährt werden: Dazu gehören z.B.

- Leistungen der häuslichen Krankenpflege, nur soweit sie nicht durch im Haushalt lebende Personen sichergestellt werden kann ( $\$ 37$ SGB V),

- Psychiatrische Krankenpflege,

- Pflegeleistungen nach SGB XI als Sach- oder Geldleistungen,

- Soziotherapie (indikationsabhängig).

Diese Optionen reichen für Menschen mit Behinderung, deren Selbstversorgungskompetenzen stark eingeschränkt sind, jedoch meist nicht aus. Beeinträchtigungen mit der Notwendigkeit der Unterstützung bei der Gesundheitssorge lassen sich vorwiegend im Lebensbereich Selbstversorgung (ICF d570ff.) bei den Aktivitäten und der Teilhabe abbilden. 
Insofern besteht hier ein Anspruch auf Leistungen der Eingliederungshilfe. Leistungsrechtlich fallen diese in den Bereich der Assistenzleistungen nach $\$ \$ 78$, 113 Abs. 1 Nr. 2 SGB IX. (Schmidt-Ohlemann 2018; DVfR 2018 b) Dort ist ausdrücklich als Leistungsbestandteil die Sicherstellung der Wirksamkeit der ärztlichen und ärztlich verordneten Leistungen benannt. Dazu gehören insbesondere:

1. Medikamentengabe,

2. Einfache Behandlungspflege,

3. Umsetzung der Ernährungsvorgaben, z.B. Trinkmenge, Bilanzierung, $\mathrm{NaCl}$ Reduktion, PEG, Zusatznahrung,

4. Organisation und Mitwirkung bei der Hilfsmittelversorgung (Sanitätshaus),

5. Nutzung von und Training mit individuellen Hilfsmitteln im Alltag (Korsett, Schienen, Schuhe: Laufen etc.),

6. Gewährleistung der Inanspruchnahme von Physiotherapie, Ergotherapie, Logopädie, Podologie, Ernährungsberatung, sofern ärztlich verordnet ggf. einschließlich Beförderung,

7. Umsetzung der Empfehlungen der therapeutischen Disziplinen zur Umsetzung im Alltag, z.B.: Bewegungsübungen, Steh- und Gehübungen, Aktivitäten des täglichen Lebens zur Anwendung des in der Therapie erlernten oder zur Sicherung der Erreichung therapeutischer Ziele,

8. Sprech- und Sprachübungen,

9. Nutzung der unterstützten Kommunikation im Alltag,

10. Beobachtung im Hinblick auf spezielle Krankheitssymptome (z.B. Anfälle) und Überwachung der Zielerreichung im Alltag (Schmerztagebuch), Blutdruck, Puls, ggf. auch in der Nacht,

11. Organisation der Kontrolluntersuchungen und Umsetzung ärztlicher Überweisungen,

12. systematisches Screening im Alltag nach spezifischen Risiken: z.B. Decubitus bei Querschnittslähmung,

13. stützende und helfende Gespräche bei psychischen Erkrankungen.

Die komplexe Behandlungspflege hingegen gehört nach der Rechtsprechung des Bundessozialgerichts (BSG) nicht zur Eingliederungshilfe, sondern zur Krankenversorgung nach $\$ 37$ SGB V. Dementsprechend ist sie in allen Landesrahmenverträgen, die nach der neuen Gesetzgebung geschlossen wurden, als Leistung der Eingliederungshilfe ausdrücklich ausgeschlossen. Da bislang auch die Behandlungspflege zu den Aufgaben insbesondere von stationären Einrichtungen der Eingliederungshilfe gehörte, müssen diese nun dafür sorgen, dass anerkannte Pflegedienste die komplexe Behandlungspflege erbringen und diese mit den Krankenkassen abrechnen können. Wenn Menschen mit Behinderungen auf Leistungen von Pflegediensten angewiesen sind, müssen sie sich auch deren organisatorischen Vorgaben anpassen. Dies kann eine erhebliche Barriere für die Teilhabe bedeuten. 
Hier bieten die Überlegungen der Fachverbände für Menschen mit Behinderung zur Durchführung der Behandlungspflege, die im April 2020 zur Diskussion gestellt wurden, Lösungsmöglichkeiten (Fachverbände 2020).

Es ist aber zu beobachten, dass den Unterstützungsbedarfen bzgl. der Gesundheitssorge bei der Bedarfsermittlung bislang wenig Aufmerksamkeit geschenkt wurde, insbesondere im Hinblick auf die Bewältigung von akuten oder chronischen Krankheiten. Dies zeigt sich u.a. bei den Bedarfserhebungsinstrumenten der Länder und wird von Betroffenen und Experten bei vielen Teilhabe- bzw. Gesamtplänen bestätigt. Es ist demnach nicht durchgängig gewährleistet, dass Unterstützungsleistungen für die Gesundheitssorge in ausreichendem Maße zur Verfügung gestellt werden. (DVfR 2018 a und b; Schmidt-Ohlemann 2018, 2019) Die mangelnde Unterstützung bei der Gesundheitssorge ist eine wesentliche Barriere bei der Inanspruchnahme des Gesundheitswesens. Dies gilt gerade auch für Menschen, die in Einrichtungen der Eingliederungshilfe leben bzw. im ambulanten Setting Leistungen der Eingliederungshilfe in Anspruch nehmen. Offen ist auch, ob die komplexe Behandlungspflege im Rahmen der Neuregelungen tatsächlich gewährleistet werden kann, ohne dass die soziale Teilhabe beeinträchtigt wird. Von der mangelnden Unterstützung bei der Gesundheitssorge sind im Übrigen auch ältere Menschen betroffen, die in Pflegeeinrichtungen leben. So wird die fachärztliche Versorgung dort häufig nicht ausreichend unterstützt, ebenso eine ausreichende Heil- und Hilfsmittelversorgung. Rehabedarfe werden dort oft nicht erkannt. (Janssen 2018) Auch wird in Fällen komplexer erworbener Schädigungen ein Fallmanagement vermisst.

Im Grunde dürften solche Barrieren nicht bestehen. Denn es besteht die Möglichkeit, dass die Betroffenen bei der Gesundheitssorge durch die Eingliederungshilfe unterstützt werden, wenn die Voraussetzungen, insbesondere das Vorliegen einer wesentlichen Behinderung gegeben sind. Denn sie haben einen Anspruch auf Leistungen der Assistenz, d.h. Leistungen z.B. nach $\$ 78$ SGB IX in Verbindung mit $\$ 113 \mathrm{ff}$. SGB IX. Dabei sind die notwendigen Leistungen bei der Bedarfsermittlung und Teilhabeplanung (u.a. $\$ \$ 13,19$ und $117 f f$. SGB IX) zu berücksichtigen. (DVfR 2018a)

Da nicht erkennbar ist, dass die Einrichtungen und Dienste der Eingliederungshilfe und insbesondere die Träger der Eingliederungshilfe dieser Verantwortung vollumfänglich gerecht werden, besteht hier wohl auch zukünftig eine große Barriere. Insofern können Probleme der Gesundheitsversorgung nicht ausschließlich im Gesundheitssystem verortet werden. Die mangelnde Inanspruchnahme von Leistungsangeboten der Gesundheitsversorgung einschließlich der Überwindung der dort vorhandenen Barrieren liegt auch an der mangelnden Unterstützung bei der Gesundheitssorge. Die Verantwortung dafür kann nur dann an Angehörige oder andere Bezugspersonen delegiert werden, wenn diese vorhanden und dazu in der Lage sind. Dies ist jedoch für Menschen, die in Einrichtungen leben, im Hin- 
blick auf die alltäglichen Anforderungen in der Regel nicht möglich. Die meisten Unterstützungsleistungen können zudem nicht dem Aufgabenkreis der gesetzlichen Betreuung zugeordnet werden.

\section{Zusammenfassung}

Barrieren bei der Gesundheitsversorgung sind vielfältig und betreffen nicht nur den Zugang, sondern auch Angebotsdefizite sowie die mangelnde Unterstützung bei der Gesundheitssorge. Die vorgetragenen Überlegungen lassen sich in folgenden Thesen zusammenfassen:

1. Es mangelt an der Unterstützung bei der Gesundheitssorge.

2. Menschen mit Behinderungen sind durch ihre Behinderung von manchen Leistungen praktisch ausgeschlossen (z.B. Zugangskriterien zur Rehabilitation bei intellektuellen Beeinträchtigungen oder mehrfachen Behinderungen bzw. Pflegebedürftigkeit).

3. Barrieren bestehen beim Zugang, während der Inanspruchnahme und bei der Wahl der Therapieoptionen.

4. Bei bestimmten behinderungsassoziierten Problemkonstellationen sind spezialisierte Einrichtungen und Dienste erforderlich: Ihr Fehlen bedeutet eine wesentliche Barriere, wenn eine bedarfsgerechte Gesundheitsversorgung im Sinne des Art. 25 UN-BRK sichergestellt werden soll.

5. Ein umfassendes Fallmanagement bei Leistungen zur Teilhabe für Menschen mit komplexen Beeinträchtigungen ist regelhaft nicht verfügbar. Die Einrichtungen und Dienste der Eingliederungshilfe und der Pflege nehmen diese Aufgabe oft nur unzureichend wahr.

6. Spezialisierte Einrichtungen sind keine Sondereinrichtungen, die auf Grund von Barrieren im Gesundheitswesen geschaffen werden, sondern sind Ausdruck einer fachlich gebotenen Spezialisierung.

7. Dennoch darf die Gefahr nicht übersehen werden, dass besondere Einrichtungen auch als Kompensation von Barrieren genutzt werden können, wobei das Gesundheitswesen ohnehin reich an Barrieren für alle Bürger ist.

8. Die erforderlichen mannigfaltigen Interventionen zum Barriereabbau sollten auch mit Hilfe der Teilhabeforschung identifiziert, geplant und evaluiert werden.

9. Die Gesundheitssorge sollte durch die Eingliederungshilfe und die Pflege umfassend unterstützt werden, soweit das primäre soziale Netz dies nicht leisten kann.

\section{Literatur}

Bundesarbeitsgemeinschaft für Rehabilitation (BAR) (Hrsg.) (2012): Die 10 Gebote der Barrierefreiheit in 10 Kernpunkten. Frankfurt. Online unter: https:/www.bar-frankfurt.de/fileadmin/dateiliste/publikationen/arbeitsmaterialien/downloads/BARBro10Gebote5.pdf. (Abrufdatum: 20.2.2020). 
Degener, Th., Diehl, E. (Hrsg.) (2015): Handbuch Behindertenrechtskonvention. Teilhabe als Menschenrecht - Inklusion als gesellschaftliche Aufgabe. Bonn: bpb-Schriftenreihe Band 1506.

Deutsche Vereinigung für Rehabilitation (DVfR) (2018a): Positionspapier der DVfR zur Umsetzung des Bundesteilhabegesetzes (BTHG): Zum Verfahren der Bedarfsermittlung und -feststellung in der Praxis und zur Bedeutung von $\$ 13$ SGB IX. Online unter: www.dvfr.de. (Abrufdatum: 21.06.2020).

Deutsche Vereinigung für Rehabilitation (DVfR) (2018b) Stellungnahme der DVfR zu Inhalten der Bedarfsermittlung: Morbidität, Sorge um Gesundheiterhaltung und Krankheitsbewältigung. Online unter: www.dvfr.de. (Abrufdatum: 21.06.2020).

Deutsche Vereinigung für Rehabilitation (DVfR) (2020): Positionspapier „Pflege und Rehabilitation“ im Erscheinen. Online unter: www.dvfr.de. (Abrufdatum: 21.06.2020).

Fachverbände für Menschen mit Behinderung (2020). Diskussionspapier: Behandlungspflege in Einrichtungen/gemeinschaftlichen/besonderen Wohnformen für Menschen mit Behinderung Stand: 18.11.2019. Online unter: https://www.reha-recht.de/infothek/beitrag/artikel/die-fachverbaendestellen-fragen-der-behandlungspflege-auf-reha-rechtde-zur-diskussion. (Abrufdatum: 20.4.2020).

Janssen, H. (2018): Ermittlung des allgemeinen Rehabilitationsbedarfs und Evaluation Mobiler Geriatrischer Rehabilitation in stationären Pflegeeinrichtungen und der Kurzzeitpllege. Studie im Auftrag des BMG. Online unter: www.bundesgesundheitsministerium.de/fileadmin/Dateien/5_Publikationen/ Pflege/Berichte/Schlussbericht_MoGeRe_10._Sept_2018.pdf. (Abrufdatum: 14.6.2019).

Martin, P. (2019): Medizin für Menschen mit intellektueller Beeinträchtigung. Eine besondere Herausforderung für das Gesundheitswesen. In: Dtsch Arztebl Int 2019; 116: 807-8;

Sappok, T, Diefenbacher, A \& Winterholler, M (2019): Medizinische Versorgung von Menschen mit Intelligenzminderung. In: Dtsch Arztebl Int 2019; 116: 809-16.

Schmidt-Ohlemann, M. (2019): Gesundheitsversorgung, medizinische Rehabilitation und Teilhabeförderung durch Einrichtungen und Dienste der Eingliederungshilfe. In: Rehabilitation 2019; 58 (04), 221-224. Stuttgart New York: Georg Thieme Verlag.

Schmidt-Ohlemann, M. (2018): Ist die Sicherstellung der medizinischen Versorgung von Menschen mit Behinderungen eine Teilhabeleistung? In: sozialpsychiatrische informationen 48. Jahrgang 4/2018, 14-19.

Schmidt-Ohlemann, M. (2017a): Medizinnahe Leistungen als Bestandteil der Teilhabeförderung. Online unter: https:/www.diefachverbaende.de/files/veranstaltungen/2017-01-20-MatthiasSchmidt-Ohlemann.pdf. (Abrufdatum: 19.2.2020).

Schmidt-Ohlemann, M (2017b): Gesundheitsbezogene Eigenverantwortung, Aktivitäten der Versicherten - Beeinträchtigungen und Ressourcen bei Menschen mit kognitiven Beeinträchtigungen oder mit chronischen seelischen Erkrankungen. Vortrag auf der Fachtagung „Gesundheitsbezogene Aufgaben in der Eingliederungshilfe - Herausforderungen für Dienste und Einrichtungen“ 20.01.2017 Kassel. Online unter: https://www.diefachverbaende.de/files/veranstaltungen/201701-20-Gesundheitssorge-Schmidt-Ohlemann.pdf. (Abrufdatum: 20.2.2020).

Schmidt-Ohlemann, M. (2014): Medizinische Zentren für erwachsene Menschen mit geistiger oder mehrfacher Behinderung (MZEB) - ein neues Element der Gesundheitsversorgung im Koalitionsvertrag. In: Recht und Praxis der Rehabilitation Heft 2/2014, 26-39.

Seidel, M. (Hrsg.) (2015): Grundsätzliche und spezielle Aspekte der gesundheitlichen Versorgung von Menschen mit geistiger Behinderung. Dokumentation der Fachtagung am 27. Februar 2015 v. Bodelschwinghsche Stiftungen Bethel. Materialien der DGSGB Band 35 Berlin. Online unter: https://dgsgb.de/downloads/materialien/Band35.pdf. (Abrufdatum: 20.2.2020).

Welti, F. (2016a): Zugänglichkeit und Barrierefreiheit der gesundheitlichen Infrastruktur - rechtliche Anforderungen - Teil 1; Beitrag D7-2016, 09.03.2016. Online unter: www.reha-recht.de (Abrufdatum: 21.06.2020).

Welti, F. (2016b): Zugänglichkeit und Barrierefreiheit der gesundheitlichen Infrastruktur - rechtliche Anforderungen - Teil 2; Beitrag D8-2016, 10.03.2016. Online unter: www.reha-recht.de (Abrufdatum: 21.06.2020). 
Leonora Micah Jordan

\section{Barrierefreie Beratung - Räume der Begegnung niedrigschwellig gestalten}

\section{Einführung und Zielsetzung des Beitrags}

Beratung stellt einerseits eine spezielle Form der Unterstützung und Begleitung von einzelnen Personen und Gruppen dar, andererseits auch ein Handlungskonzept (vgl. Klein 2010, 34). In variantenreichen Ausrichtungen, Ansätzen, Methoden und Konzepten sowie unterschiedlicher thematisch-inhaltlicher Schwerpunktsetzung unterliegt Beratung sich verändernden internen und externen Anforderungen, wie andere personen- und strukturbezogene Dienstleistungen auch. Diese Kommunikationsform der professionellen Intervention - mit unterschiedlichem Institutionalisierungs- und Formalisierungsgrad - findet Anwendung sowohl in gesundheitsberuflichen, pflegerischen, psychosozialen, (sozial-) pädagogischen und soziotherapeutischen Arbeitsfeldern als auch in Organisationsentwicklung und -management (vgl. Engel u.a. 2014, 34).

Eingebettet in (haushalts-)rechtliche, ökonomische, institutionelle und berufsethische Rahmenbedingungen ist Beratung subjektorientiert, aufgabenspezifisch und kontextbezogen. Auch wenn Adressat*innenkreise und Beratungsfelder, -methoden und -anliegen oder Beratungssettings und -konstellationen variieren, so werden Ratsuchende bei der Reflexion von Erfahrungen unterstützt und/oder befähigt, eigenverantwortlich Entscheidungen treffen zu können, Problem- und Konfliktbewältigungsstrategien zu entwickeln, Selbstermächtigungspotentiale und soziale Ressourcen in unterschiedlichen Teilhabebereichen - etwa lebensweltlichen oder arbeitsweltlichen Bezügen - zu erschließen (vgl. Arbeitsgemeinschaft Beratungswesen 2014, 1276ff.).

In diesem Beitrag werden die Bedeutung von Barrierefreiheit im Kontext von Beratung herausgearbeitet und Hinweise zur Umsetzung gegeben. Anschließend wird auf Dimensionen von Niedrigschwelligkeit eingegangen. 


\section{Zugangsbedingungen zu Beratung}

Anfang der 2000er Jahre wird das Verständnis von Barrierefreiheit auch auf die Zugänglichkeit und Verfügbarkeit sozialer Güter und Dienstleistungen - somit auch auf Beratungsangebote - übertragen. Neben räumlichen Barrieren (wie Treppenstufen, Schwellen, schmalen Türen, schwer passierbaren oder ungesicherten Fußgängerüberwegen) werden weitere segmentierende Hürden und Selektionsmechanismen ungleicher Zugangsbedingungen zu Beratungsangeboten erkannt und beschrieben (vgl. Bellermann 2015, 54). Dem Verständnis der UN-Behindertenrechtskonvention (UN-BRK) folgend, entsteht Be-Hinderung ,aus der Wechselwirkung zwischen Menschen mit Beeinträchtigungen und einstellungs- und umweltbedingten Barrieren" (Präambel UN-BRK). Erst seit der bundesweiten Einführung von Angeboten der „Ergänzenden unabhängigen Teilhabeberatung“ $\left(\mathrm{EUTB}^{\circ}\right.$ ) im Jahr 2018 gelangen die in der UN-BRK und den Gleichstellungsund Antidiskriminierungsgesetzen konkretisierten Mindeststandards und Leitlinien zur barrierefreien Zugänglichkeit zu beratenden Einrichtungen und Diensten auch in den Fokus der Beratungsforschung.

Je nach Beratungsfeld können sich die Adressat*innengruppen unterscheiden und somit auch die zielgruppenspezifischen Bedürfnisse eines gleichberechtigten Zuganges: So bilden etwa akustische Auffindhilfen und Sprachausgaben für blinde und erheblich sehbehinderte Menschen eine wichtige Orientierungshilfe; für Menschen mit Mobilitätseinschränkungen kann eine einzige Stufe schon eine unüberwindbare Barriere darstellen, während Menschen mit Wahrnehmungsbeeinträchtigungen und Konzentrationsstörungen eine möglichst optisch, akustisch und olfaktorisch reizarme Umgebung benötigen.

Der Bedarf von Barrierefreiheit sollte nicht nur im Kontext der Arbeit mit Menschen mit Behinderung stehen, sondern universal design und design for all sollten die Grundlage für die bauliche Planung von Gebäuden, Gestaltung von Produkten und inhaltliche Ausrichtung von Dienstleistungen bilden (vgl. Skiba \& Züger 2017, 9f.). So profitieren z.B. auch Menschen mit (temporären) körperlichen Beeinträchtigungen (etwa einem Gipsbein) oder Menschen, die mit einem Kinderwagen oder unhandlichem Gepäck unterwegs sind, von stufenloser Zugänglichkeit und Aufzügen. Auffällige kontrastreiche Beschilderungen und Piktogramme stellen Orientierungshilfen für Menschen mit Orientierungs-, Lese- und Lernschwierigkeiten, einer Sehschwäche und ortsfremde Personen dar - um nur einige Beispiele zu nennen, aus welchem Grund das Konzept der Niedrigschwelligkeit mehrdimensional betrachtet werden muss (vgl. Abbildung 1). 


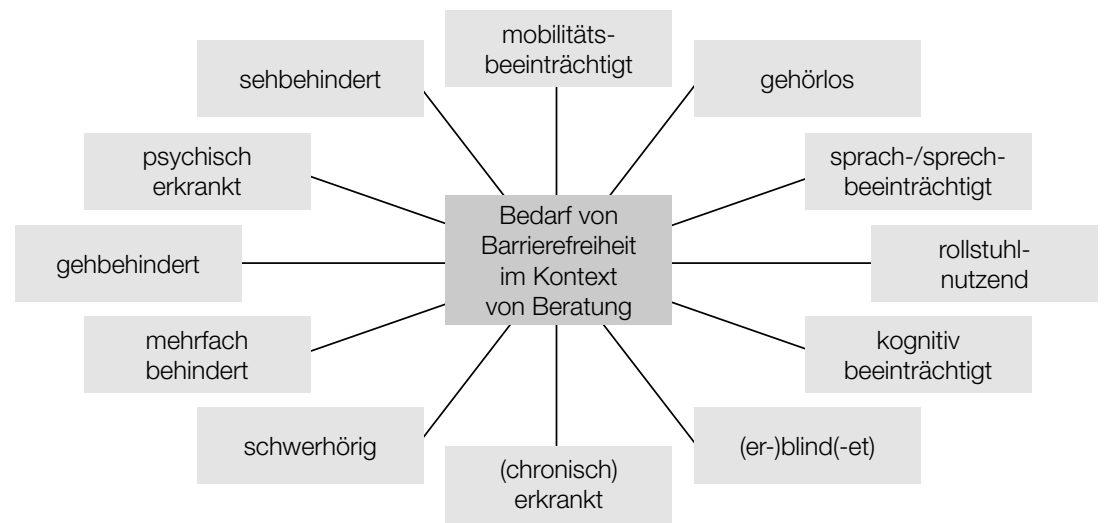

Abb. 1: Der Barrierefreiheitsbedarf im Kontext von Beratung (eigene Darstellung in Anlehnung an Skiba \& Züger 2017, 9)

\section{Was bedeutet Barrierefreiheit im Kontext von Beratung?}

Ausgehend von einem interdisziplinären Ansatz, der gesundheitsberufliche, pflegerische, psychosoziale, (sozial-)pädagogische und soziotherapeutisch ausgerichtete Beratungspraxis umfasst, ist es unübersehbar, dass die unterschiedlichen wissenschaftlichen Fachrichtungen spezifische Zugänge in Aus-, Fort- und Weiterbildung untersuchen, Professionalisierungs- und Verrechtlichungsbestrebungen unumgänglich scheinen und Maßnahmen zu Qualitätsentwicklung und -management für unterschiedliche Beratungsangebote getroffen wurden und werden. Alltags- und Lebensweltorientierung, Empowerment der Ratsuchenden und Ressourcenbezug werden neben der lebensweltnahen, alltagssensiblen und nutzerspezifischen Orientierung in der Frankfurter Erklärung zur Beratung als profilbildende Handlungsmaximen benannt (vgl. Nestmann u.a. 2014, 1272ff.).

In der Literatur zu Beratungstheorie und -forschung finden sich vielfältige Aussagen zur Bedeutung fachlicher Fundierung der Beratenden, der Qualitätssicherung von Beratung, dem jeweiligen Beratungsverständnis, Handlungskonzepten und -orientierungen sowie theoriegeleiteten Grundlagen über Beratungs- und Interaktionswissen sowie handlungsfeldspezifischem Wissen, jedoch wird bisher nur selten auf die Bedeutung von Barrierefreiheit und Niedrigschwelligkeit in der Beratung verwiesen (vgl. Engel u.a. 2014, 35).

Anknüpfend an die Diskurse zu Diversität, Intersektionalität und Ableism müssen auch Beratungsforschung und in der Beratung Aktive unter Berücksichtigung von sozialer Gerechtigkeit, Partizipation und Chancengleichheit das eigene professionelle Beratungsangebot und -handeln auf Barrierefreiheit und Niedrigschwel- 
ligkeit überprüfen. In $₫ 4$ des Gesetzes zur Gleichstellung von Menschen mit Behinderungen (Behindertengleichstellungsgesetz - BGG) wird darauf hingewiesen, dass neben baulichen Anlagen, Verkehrsmitteln und technischen Gebrauchsgegenständen auch

„Systeme der Informationsverarbeitung, akustische und visuelle Informationsquellen und Kommunikationseinrichtungen, (...) für Menschen mit Behinderungen in der allgemein üblichen Weise, ohne besondere Erschwernis und grundsätzlich ohne fremde Hilfe auffindbar, zugänglich und nutzbar (...) [sein sollen, wobei, M.J.] die Nutzung behinderungsbedingt notwendiger Hilfsmittel zulässig" ist.

Das bedeutet, dass Beratungsangebote z.B. nicht nur stufenlos erreichbar sein müssen, sondern dass - wenn vorhanden - der Aufzug groß genug ist, um ihn mit einem Elektrorollstuhl nutzen zu können, ferner sollte eine Sprachausgabe integriert sein. Gut beschilderte Sanitäranlangen - auch für rollstuhlnutzende Ratsuchende/Beratende - sollten vorhanden und zugänglich sein. Des Weiteren sollten Assistenz- und Begleithunde mitgebracht werden dürfen. Flyer sollten in Leichter Sprache vorgehalten werden, Texte auf der Homepage mit gängigen Screenreadern oder Vergrößerungssoftware gelesen werden können und das Webdesign barrierefrei zugänglich und bedienbar sein (vgl. Hellbusch 2019, 1) - denn niemand darf aufgrund seiner Behinderung benachteiligt werden (Art. 3 Abs. 3 Satz 2 GG).

Im Gegensatz zu dem eher normenorientierten Begriff der Barrierefreiheit fehlt bislang eine einheitlich-verbindliche theoretische Fundierung von Niedrigschwelligkeit. Nach Mayrhofer (2012, 10ff.) bezieht sich der metaphorische Begriff der Niedrigschwelligkeit auf die Abwesenheit möglicher Barrieren, Hürden und Schwellen. Beratungsangebote können sowohl in ihrer inhaltlich-konzeptionellen Gestaltung und sozialen Ausrichtung „niedrigschwellig“ sein als auch in der räumlichen Zugänglichkeit und Ausstattung sowie in ihrer zeitlichen Erreichbarkeit. Monetäre und administrativ-rechtliche Zugangsvoraussetzungen können weitere Hürden der Inanspruchnahme von Beratung darstellen (vgl. Konter 2019, 1).

In der Förderrichtlinie des Bundesministeriums für Arbeit und Soziales (BMAS) aus dem Jahr 2017 zur Durchführung der „Ergänzenden unabhängigen Teilhabeberatung" werden vier Dimensionen niedrigschwelliger Zugänglichkeit zu den geförderten Beratungsangeboten benannt (vgl. BMAS 2017, 1), die im Folgenden näher betrachtet werden:

1. inhaltliche Niedrigschwelligkeit,

2. räumliche Barrierefreiheit,

3. soziale Niedrigschwelligkeit und

4. zeitliche Zugänglichkeit.

\subsection{Zur inhaltlichen Niedrigschwelligkeit in der Beratung}

Insbesondere in eher informell ausgerichteten Beratungssettings bietet sich die Möglichkeit, inhaltlich niedrigschwellig zu beraten. Gerade durch - bisher wenig 
erforschte - offene Settings können, im Sinne von Verwirklichungschancen, Personengruppen erreicht und Themen erschlossen werden, welche mittels stärker formalisierten und (vor-)strukturierten Settings weniger zugänglich sind oder weniger angemessen erscheinen. Offene Beratungssettings stellen einen inhaltlich niedrigschwelligen Zugang zu Beratung dar, da hier Ratsuchende von sich aus Kontakt aufnehmen (Komm-Struktur) und somit Zeitpunkt, Dauer sowie Gesprächsinhalte selbst bestimmen (partizipatorischer Ansatz) und sie keinerlei Vorwissen, Berechtigung oder Qualifikation vorweisen müssen, Abstinenz (von stoffgebundenen und nicht-stoffgebundenen Suchtmitteln) oder anderen Voraussetzungen zu genügen haben, um Beratung in Anspruch nehmen zu können (vgl. Knab 2014, 72f.).

Die achtsam-respektvolle Akzeptanz der/des Ratsuchenden und des individuellen Lebensumfeldes erleichtert nicht nur das Fokussieren auf das Individuum mit dem persönlichen Beratungsanliegen, sondern stellt ein Kernelement niedrigschwelliger Beratung dar, wie sie seit Jahrzehnten erfolgreich in Sozialräumlichen Ansätzen von Streetwork und Mobiler Jugendarbeit praktiziert wird (vgl. Gillich 2006, 56ff.). Mitunter kann ein Beratungsziel darin bestehen, zunächst einmal die Beratungsfähigkeit an sich oder das Erkennen von Auslösern oder konfliktbefördernden Verhaltens selbst zum Gegenstand der Beratung werden zu lassen, z.B. die Förderung des Problembewusstseins - neben der Anbahnung von Lern- und Reflexionsprozessen, um Ratsuchende zu befähigen, reflektiert und informiert eigenständig Entscheidungen treffen zu können (vgl. Schwarzer \& Posse 2014, 76ff.). Insbesondere für Menschen, die sich in einem Gewissens- oder Loyalitätskonflikt befinden, können Beratende professionelle Vertrauenspersonen, Wegbereitende und Wegbegleitende darstellen, wenn sie sich offen zeigen für die Anliegen, Fragen und Sorgen der Ratsuchenden und diesen wertschätzend, zugewandt und empathisch begegnen. Ein differenziertes fachliches und informelles Wissen sowie Erfahrungs- und Handlungswissen bilden, neben einem reflektierenden kollegialen Austausch - idealerweise ergänzt durch Supervision -, eine sichere Basis für die Beratenden, um gemeinsam mit den Ratsuchenden adäquate Lösungsansätze zu entwickeln (vgl. Tiefel 2004, 35).

Abhängig von Adressat*innenkreis, kulturellem Milieu und institutioneller Anbindung kann die inhaltlich niedrigschwellige Ausrichtung unterschiedlicher Beratungsangebote deutlich variieren: So stellt eine gut ausgestattete Spielecke für Kinder in Familien- und Erziehungsberatungsstellen eine sinnvolle Ergänzung im Wartebereich dar, während es in einer Suchtberatungsstelle vielleicht hilfreicher ist, einen Aufsteller mit Flyern von Selbsthilfe-Gruppen mit unterschiedlichen Formen von Literatur zur Zerstreuung und Fachliteratur zu ergänzen. Diese sich in der Ausstattung manifestierende inhaltlich-niedrigschwellige Gestaltung unterstützt die Selbstrepräsentation eines Beratungsangebotes einerseits und kann andererseits zu einer inhaltlichen Niedrigschwelligkeit bereits im Empfangs- und Wartebereich beitragen (vgl. Großmaß 2014, 25f.). Dem (gesetzlichen) Auftrag 
an Barrierefreiheit in der Beratung folgend, sollten Flyer, Informationsbroschüren und -materialien möglichst auch in Leichter Sprache, serifenlosem Großdruck und in Screenreader-angepasstem digitalen Format verfügbar sein.

Inhaltlich niedrigschwellige Beratung kann dazu beitragen, dass Ratsuchende erreicht werden, die sich bereits in prekären Lebenssituationen befinden (oder drohen, in diese abzugleiten), von gesellschaftlichen Ausgrenzungsstrukturen betroffen sind und bei Nichtinanspruchnahme professioneller Beratung evtl. in existentiell gefährdende Notlagen geraten können (vgl. Konter 2019, 1).

\subsection{Aspekte der räumlichen Barrierefreiheit}

In seiner Beschreibung von „Ortseffekten“ weist Pierre Bourdieu (1997) darauf hin, dass sich soziale Strukturen einer Gesellschaft auch immer geografisch abbilden. Dieses trifft neben administrativen Einrichtungen auch auf Geschäftsbereiche sowie die Segmentierung des privaten Wohnraumes zu - so auch auf die Lokalisation von Beratungsangeboten. Bei der Neuplanung von Beratungsstellen fließen in die Entscheidungen über die räumlich-geografische Lage neben pragmatisch-ökonomischen Überlegungen auch die Erreichbarkeit der avisierten Adressat*innengruppe ein (vgl. Großmaß 2014, 24). Das Angebot der aufsuchenden Beratung, welche in mobiler Form außerhalb der Beratungsstelle erfolgt, kann als eine Ergänzung räumlich niedrigschwelliger Beratung erfolgen (vgl. Schürmann 2014, 526f.), wenn z.B. die Räumlichkeiten nicht barrierefrei erreichbar und/oder zugänglich sind. Insbesondere die Anbindung an den öffentlichen Personennahverkehr (ÖPNV), eine zentrale Lage der Beratungsstelle sowie eine kontrastreiche Beschilderung und die stufenlose Zugänglichkeit werden in der Literatur als zentrale Merkmale der räumlichen Niedrigschwelligkeit betont (vgl. Agentur Barrierefrei NRW 2019, 4ff.). Die Steigung von Zugangswegen und (1,20 m breiten) Rampen sollten 6\% nicht überschreiten; ist der reguläre Zugangsweg nicht stufenlos, sollten Hinweise zum barrierefreien Eingang gut sichtbar sein, und idealerweise sind Türen - mit einer Mindestbreite von $90 \mathrm{~cm}$ - mit einem automatischen Türöffner versehen. Unvermeidbare Hindernisse sollten kontrastreich markiert sein (wie etwa die erste und letzte Treppenstufe); Treppen sollten mit einem Handlauf versorgt sein, der von der ersten bis zur letzten Stufe reicht; Aufzüge sollten mit Sprachausgabe versehen sein, ein niedrig angebrachtes taktiles Bedienfeld haben und eine Grundfläche von $1,10 \mathrm{~m}$ mal 1,40 m aufweisen (vgl. Agentur Barrierefrei NRW 2019, 4ff.). Bei der Erstellung einer Anfahrtsbeschreibung sollte darauf geachtet werden, dass nicht nur die nächstgelegenen Haltestellen mit den jeweiligen Liniennummern angeführt werden, sondern dass ggf. auf die (Nicht-)Barrierefreiheit hingewiesen wird und mögliche Gefahrenstellen benannt werden, wie eine stark befahrene Straße mit extrem kurzer Grünphase der nächstgelegenen Lichtsignalanlage, nicht abgesenkte Bordsteine an Kreuzungen, Gefälle über 9\%, Engstellen, Querneigungen über 3\% o.Ä. Auch auf (nicht) vorhandene Bodenindikatoren und Leitsysteme sollte hingewiesen werden. Das 
Abdrucken einer Umgebungsskizze stellt eine visuelle Orientierungshilfe dar. Auf naheliegende (Behinderten) Parkplätze sollte hingewiesen werden. Ebenso können Geschäfte mit starken olfaktorischen Reizen, wie Bäckerei oder Parfümerie, in die Wegbeschreibung integriert werden (vgl. Deutsche Rentenversicherung Knappschaft-Bahn-See 2019, 9).

Neben der barrierefreien Zugänglichkeit zu einem Empfangsbereich sollten auch Raumausstattung und-gestaltung der abgetrennten Beratungsräume weitgehend barrierefrei gehalten sein, d.h., es sollte hinreichender Bewegungs- und Verkehrsraum in Fluren, Beratungsräumen und Sanitärräumen vorhanden sein; Tische sollten höhenverstellbar sein und auf hinreichende Beinfreiheit geachtet werden; die Bestuhlung sollte beweglich sein, so dass bei Bedarf Sitzgelegenheiten für eine oder mehrere Begleitperson/en vorhanden sind oder ggf. hinreichend Platz für Ratsuchende (oder Beratende) mit Mobilitätshilfen, wie Rollstuhl, Rollator oder Gehhilfen, gegeben ist. Die Raumbeleuchtung sollte dimmbar sein, Schreib- und Leseflächen sollten jedoch gut ausleuchtbar sein, ebenso der Sitzplatz für etwaige Gebärdensprach- und Schriftdolmetschende oder Lormende (vgl. DIN 18024-2: 1996-11).

Ferner ist die Raumakustik der Innenraumgestaltung zuzuordnen: Die Nachhallzeit kann durch das Anbringen von Schallabsorbtionselementen an Wänden und unter der Zimmerdecke, Bücherregalen an gegenüberliegenden Wänden oder fest verlegten Teppichboden reduziert werden; Hörgerät-Nutzende profitieren von installierten induktiven Höranlagen in größeren Besprechungsräumen. Auf reflektierende, glänzende Oberflächen sollte verzichtet werden, wie auch auf übermäßige Dekoration und Raumdüfte, um in reizarmer Umgebung nicht von Beratungsinhalten abzulenken (vgl. DIN 18024-2: 1996-11).

Zumindest ein rollstuhlbefahrbares WC sollte vorhanden sein; wichtig ist hier, ausreichende Bewegungsfläche (1,20 m mal 1,20 m) zu berücksichtigen, die Unterfahrbarkeit des Waschtisches zu gewährleisten, automatische oder Einhandhebel-Armaturen zu verbauen und (schwenkbare) Stütz-, Hebe- und Umsetzhilfen so anzubringen, dass Verkehrswege nicht beeinträchtigt werden. Auf Spülauslösung und Toilettenpapier sollten sitzend im vorderen Bereich zugegriffen werden können (vgl. Richtlinie VDI 6000 Blatt 3).

In den Technischen Bauvorschriften des Deutschen Instituts für Bautechnik (DIBT) wird darauf hingewiesen, dass Planungsgrundlagen, Normen, Richtlinien und Empfehlungen für barrierefreies Bauen sich am aktuellen Stand baulichtechnischer Möglichkeiten orientieren und diese erst rechtsverbindlich wirksam werden durch entsprechende Gesetze und Verordnungen der Länder, wie z.B. den Technischen Baubestimmungen und Landesbauordnungen (vgl. DIBT 2019, 1).

\subsection{Zur sozialen Niedrigschwelligkeit}

Böhnisch (2012) legt dar, dass Lebens- und Problembewältigungskompetenzen u.a. mit der Bildung des Individuums in Zusammenhang stehen. Ein inhaltlich 
niedrigschwelliges Beratungsangebot - wie in oben beschriebenen offenen Settings - kann hier einen deutlichen Beitrag zu sozialer Gerechtigkeit leisten, da so auch gesellschaftlich marginalisierte (häufig zudem „bildungsferne“) Randgruppen Zugang zu Beratungs- und Unterstützungsleistungen erhalten können. Die Möglichkeit, ohne zuerst administrative Hürden in Form von Terminabsprache, Voranmeldung oder der Beantragung einer Beratungsleistung in offenen Settings beraten zu werden, bildet soziale Zugangsgerechtigkeit und Chancengleichheit in doppelter Hinsicht ab: Mittels eines sozial niedrigschwelligen Beratungsangebotes werden Zugangswege zu anderen Hilfe- und Unterstützungssystemen eröffnet, was wiederum eine Steigerung der Teilhabemöglichkeiten am gesellschaftlichen-, kulturellen oder Arbeitsleben bedeuten kann (vgl. Knab 2014, 72).

Neben dem klassischen Setting der Face-to-face-Beratung in einem physischen Beratungsraum oder per Telefon eröffnen barrierefrei nutzbare mediale Kommunikationssysteme die Möglichkeit, virtuell miteinander in Kontakt zu treten. Ratsuchende, die - aus welchen Gründen auch immer - nicht hinreichend mobil sind, um eine Beratungsstelle aufzusuchen, können in Form der Online-Beratung per Chat, E-Mail oder Video-Konferenz trotzdem sinnvoll unterstützt und begleitet werden.

Schutz und Wahrung der Anonymität von Ratsuchenden stellt einen weiteren Aspekt sozialer Niedrigschwelligkeit dar, d.h., auf Wunsch sollte es möglich sein, Menschen zu beraten, ohne dass sie ihren Namen nennen müssen und ohne dass Telefonnummer/IP-Adresse entsprechend der geltenden Datenschutzrichtlinien gespeichert oder sonstige Datenspuren ohne Zustimmung der Ratsuchenden aufgezeichnet werden. Gerade wenn die Wahrung der Anonymität der Ratsuchenden von Bedeutung ist, kann auf telefonischen und virtuellen Kommunikationswegen das Risiko der persönlichen Identifizierung minimiert werden; gleichzeitig wird bei diesen Kommunikationsformen der ,geteilte körperlich erfahrbare Wahrnehmungs- und Handlungsraum außer Kraft gesetzt“, merkt Engel $(2014,504)$ an. Wenn die Beratungsanliegen sensiblen Gesundheitsthemen zuzuordnen oder tabubehaftet sind (z.B. Informationen zum Umgang mit HIV, Schwangerschaftskonfliktberatung, Suizidalität oder sexualisierte Gewalt) oder die ratsuchende Person akut von Gewalt betroffen ist (etwa häusliche Gewalt oder Zwangsprostitution), kann die niedrigschwellige telefonische oder OnlineErreichbarkeit eines Beratungsangebotes u.U. lebensrettend sein. Nicht ohne Grund sind z.B. bundesweite Rufnummern der Telefonseelsorge tags wie nachts durchgängig besetzt, seit 1995 ergänzt um die Möglichkeit des Chattens und des E-Mail-Austausches.

Auch die Wahrung der Schweigepflicht gegenüber Dritten ist der sozialen Niedrigschwelligkeit von Beratung zuzuordnen, insbesondere wenn personenbezogene Daten erhoben werden, die Gesundheit, Arbeits-, Wohn- oder Lebenssituation, Sucht, Sexualität oder Vermögen betreffen (vgl. $\$ 203$ StGB). 
Eine allgemeine Offenheit und Ansprechbarkeit für alle Problembereiche und $A k$ zeptanz aller Ratsuchenden in ihrer jeweiligen Situation ist - nicht nur vor dem Hintergrund des Allgemeinen Gleichbehandlungsgesetzes (AGG) - wünschenswert.

Die Möglichkeit, kostenlose Beratung in Anspruch zu nehmen, ist natürlich abhängig von der jeweiligen institutionellen Anbindung und konzeptionellen Ausrichtung, ist aber im Sinne einer barrierefreien sozialen Zugänglichkeit zu begrüßen. Gebühren oder Honorare sollten eine Inanspruchnahme dringend benötigter Beratung nicht verhindern oder eine unüberwindbare monetäre Barriere darstellen.

\subsection{Dimensionen zeitlicher Zugänglichkeit}

Die Erreichbarkeit zu üblichen Geschäftszeiten kann sinnvoll ergänzt werden durch das Angebot einer offenen Sprechstunde, wo Ratsuchenden eine spontane (erste) Kontaktaufnahme ermöglicht wird. Ergebnisse einer Untersuchung zur Erziehungs- und Familienberatung sprechen diesem konzeptionellen Ansatz eine deutliche Senkung der Zugangsschwelle zu (vgl. Menne 2001, 55). Erwerbstätige Ratsuchende - insbesondere im Schichtdienst Beschäftigte - begrüßen die Möglichkeit einer Abendsprechstunde, d.h., es können auch Beratungstermine in den späten Nachmittags- oder frühen Abendstunden vereinbart werden.

Neben telefonischen Ansprechzeiten, festen Bürozeiten und der (zeitlich unabhängigen) Kontaktaufnahme per E-Mail betonen Ratsuchende die Bedeutung von komplementären niedrigschwelligen Angeboten, die in den Räumen der Beratungsstelle stattfinden, wie etwa „Offene Cafés“, Spielenachmittage oder organisierte Freizeit-Treffen, da so ein unverbindlicher Erstkontakt nicht nur mit anderen „Peers“, sondern auch mit Mitarbeitenden - ggf. auch anwesenden Beratenden der Beratungsstelle aufgenommen werden kann (vgl. Braukmann u.a. 2017, 83). Die zeitnahe Bearbeitung von Anfragen Ratsuchender ist ebenso der zeitlichen Dimension von Niedrigschwelligkeit in der Beratung zuzuordnen wie die Möglichkeit, in einem überschaubaren Zeitraum einen Beratungstermin zu erhalten. Als Orientierungshilfe kann hierzu das Terminservice- und Versorgungsgesetz (TSVG) herangezogen werden: Seit Anfang 2020 darf die Wartezeit auf einen Termin bei einem niedergelassenen Arzt maximal eine Woche betragen, für psychotherapeutische Akutbehandlung maximal zwei Wochen. Des Weiteren wird das Mindestsprechstundenangebot der niedergelassenen Vertragsärzte auf 25 Stunden pro Woche (inklusive Hausbesuche) festgesetzt. Gewisse Facharztgruppen müssen mindestens fünf Stunden wöchentlich in Form der offenen Sprechstunde anbieten (vgl. Bundesgesetzblatt 2019, 690). Zudem sind die Terminservicestellen zu jeder Tages- oder Nachtzeit barrierefrei telefonisch, online und via App erreichbar, um eine Terminvergabe zu ermöglichen (vgl. ebd., 652). Obgleich es sich bei Beratungsanliegen i.d.R. nicht um möglicherweise akut lebensbedrohliche Gesundheitszustände handelt - abgesehen von notfallpsychologischer Beratung, Kri- 
sen- und Suizidberatung, psychotraumatologischer Beratung, Schwangerschaftskonfliktberatung u.Ä. -, so darf dennoch nicht der individuelle Leidensdruck der Ratsuchenden vernachlässigt werden, was die o.a. Terminfenster auf das Beratungsfeld übertragbar werden lässt. Orientiert am bio-psycho-sozialen Krisenverständnis kann dringend gewünschter Beratungsbedarf dem dringlichen Wunsch einer Arztkonsultation entsprechen (vgl. Wüllenweber 2009, 26ff.).

Im Gegensatz zu anerkannten psychotherapeutischen Verfahren unterliegen Beratungsdauer, häufigkeit und -intensität keinen gesetzlichen Beschränkungen, so dass die Beratenden lediglich internen personellen Strukturen, konzeptionellmethodischen Ausrichtungen und der hausinternen Kostenverantwortung des jeweiligen Trägers/Arbeitgebers unterliegen; auch der rechtliche Status der Ratsuchenden ist nachrangig.

\section{Schlussfolgerungen}

Die Beratungstätigkeit an sich stellt eine stark personalisierte Möglichkeit der Intervention dar, was an die Beratenden nicht nur auf professionell-fachlicher Ebene hochkomplexe Kompetenzansprüche stellt, sondern auch an die sozial-psychologisch-emotionalen Fähigkeiten der professionellen Beziehungsgestaltung. Nicht nur die aktive Gestaltung von Kontaktaufbau, Etablierung einer vertrauensbasierten Beratungsbeziehung, Herstellung und Ausbalancieren von Nähe und Distanz berühren eigene Identitätsvorstellungen und persönliche Kompetenzen der Beratenden - wenn zudem noch die oben beschriebenen Aspekte niedrigschwelliger Beratung berücksichtigt werden, so erfordert dieses eine bewusste Auseinandersetzung mit vielfältigen Formen von Barrieren/Hindernissen/Einschränkungen/ Be-Hinderungen, die offen oder versteckt, explizit oder implizit vorhanden sind. Jochen Schweitzer, ein erfahrener Lehrtherapeut, Supervisor für Systemische Therapie und Beratungsforscher, stellt fest, dass der (therapeutisch-)beraterische Kompetenz- und Wissenserwerb keinesfalls allein durch Fort- und Weiterbildung geschieht, sondern zugleich auf anderen - teils auch informellen - Wegen erfolgt (vgl. Schweitzer 2018, 10).

Der vorliegende Beitrag soll der Sensibilisierung für die Mehrdimensionalität von „Barrierefreiheitsbedarfen“ in der Beratung dienen. Allein die Bedürfnisse einer „barrierefreien Beratung“ bedeutet - auf der räumlichen Ebene - für junge Ratsuchende mit Mobilitätseinschränkung etwas gänzlich anderes als für gehörlose Ratsuchende im höheren Lebensalter, kann aber im Hinblick auf die soziale oder zeitliche Niedrigschwelligkeit ähnlich sein. Für einen stark depressiven Mann ohne festen Wohnsitz oder eine traumatisierte Frau mit Fluchterfahrung sind räumliche Hindernisse möglicherweise nachrangig, jedoch soziale und inhaltiche Barrierefreiheit bedeutsamer. Erkenntnisse der Intersektionalitätsforschung zu Entstehung 
und Zusammenwirken verschiedener Diskriminierungsformen in einer Person sind auch auf den Beratungssektor übertragbar. Demnach muss auch im Beratungskontext nicht nur „Behinderung“, sondern auch „Barrierefreiheit" unter der Perspektive von Intersektionalität, Diversität und Ableism neu gedacht werden. Als zentrales Element der Berater*innenausbildung stellt Reinkraut (2009, 19) heraus: „Critical thinking, multiple perspectives, and constructive developmental mean-making are key components of our academic approach to counselor training."

\section{Literatur}

Agentur Barrierefrei NRW am Forschungsinstitut Technologie und Behinderung (FTB) (Hrsg.) (2019): Barrierefreiheit in öffentlich zugänglichen Gebäuden. Lösungsbeispiele für Planung und Beratung unter Berücksichtigung der DIN 18040-1. 5. Aufl. Online unter: http://www.ab-nrw.de/ images/stories/download/2019/broschuere_barrierefreies-bauen_rz_2019-05-09_online_es_nbf. pdf. (Abrufdatum: 14.11.2019).

Arbeitsgemeinschaft Beratungswesen (2003): Psychosoziales Beratungsverständnis. In: F. Nestmann; F. Engel \& U. Sickendieck (Hrsg.) (2014): Das Handbuch der Beratung. Bd. 2: Ansätze, Methoden und Felder. 3. Aufl. Tübingen: dgvt, 1275-1280.

Arnold, H. \& Höllmüller, H. (Hrsg.) (2017): Niederschwelligkeit in der Sozialen Arbeit. Weinheim: Beltz.

Bellermann, M. (2015): Sozialstaatliche Rahmenbedingungen in Deutschland. In: B. Wüthrich, J. Amstutz \& A. Fritze (Hrsg.): Soziale Versorgung zukunftsfähig gestalten. Wiesbaden: VS Verlag für Sozialwissenschaften, 53-62.

BMAS - Bundesministerium für Arbeit und Soziales (Hrsg.) (2017): Förderrichtline zur Durchführung der „Ergänzenden unabhängigen Teilhabeberatung“ für Menschen mit Behinderungen - vom 17. Mai 2017. Online unter: https://www.bmas.de/DE/Presse/Meldungen/2017/bmas-veroeffentlicht-foerderrichtlinie-fuer-die-ergaenzende-unabhaengige-teilhabeberatung.html. (Abrufdatum: 14.11.2019).

Böhnisch, L. (2012): Sozialpädagogik der Lebensalter. Eine Einführung. 6. Aufl. München: Beltz Juventa.

Bourdieu, P. (1997): Ortseffekte. In: P. Bourdieu (Hrsg.) (2017): Das Elend der Welt. Zeugnisse und Diagnosen alltäglichen Leidens an der Gesellschaft. Köln: Herbert von Halem, 159-167.

Braukmann, J., Heimer, A., Jordan, M., Maetzel, J., Schreiner, M. \& Wansing, G. (2017): Evaluation von Peer Counseling im Rheinland. Endbericht. Online unter: https://www.prognos.com/publikationen/alle-publikationen/782/show/e84b75f89fcb98c85694d1d10edf856b. (Abrufdatum: 14.11.2019).

Brückner, G. u.a. (2001): Frankfurter Erklärung zur Beratung - Aufruf zu einem Neuen Diskurs. In F. Nestmann, F. Engel \& U. Sickendieck (Hrsg.) (2014): Das Handbuch der Beratung. Bd. 2: Ansätze, Methoden und Felder. 3. Aufl. Tübingen: dgvt, 1271-1274.

Deutsches Institut für Bautechnik - DIBT (Hrsg.) (2019): Technische Baubestimmungen. Online unter: https://www.dibt.de/de/wir-bieten/zulassungen-etas-und-mehr. (Abrufdatum: 14.11.2019).

Deutsche Rentenversicherung Knappschaft-Bahn-See (Hrsg.) (2019): Der Bund wird barrierefrei. Die Service-Angebote der Bundesfachstelle Barrierefreiheit für Bundesbehörden. Bochum: Eigenverlag. Online unter: https://www.bundesfachstelle-barrierefreiheit.de/SharedDocs/Downloads/DE/Veroeffentlichungen/broschuere-bundesfachstelle.pdf. (Abrufdatum: 14.11.2019).

Engel, F. (2014): Beratung und Neue Medien. In: F. Nestmann; F. Engel \& U. Sickendieck (Hrsg.): Das Handbuch der Beratung. Bd. 1: Disziplinen und Zugänge. 3. Aufl. Tübingen: dgvt, 497-509. 
Engel, F., Nestmann, F. \& Sickendieck, U. (2014): „Beratung“ - Ein Selbstverständnis in Bewegung. In: F. Nestmann, F. Engel \& U. Sickendieck (Hrsg.): Das Handbuch der Beratung. Bd. 1: Disziplinen und Zugänge. 3. Aufl. Tübingen: dgvt, 33-43.

Gesetz für schnellere Termine und bessere Versorgung (Terminservice- und Versorgungsgesetz TSVG) vom 6. Mai 2019. BGBl. Teil I, 646-691.

Gillich, S. (Hrsg.) (2006): Professionelles Handeln auf der Straße. Praxisbuch Streetwork und Mobile Jugendarbeit. Gelnhausen-Roth: TRIGA - Der Verlag Gerlinde Heß.

Großmaß, R. (2014): Arbeit im „Zwischen“ - zur gesellschaftlichen Verortung von Beratung. In: I. Melter, E. Kanelutti-Chilas \& W. Stifter (Hrsg.): Zukunftsfeld Bildungs- und Berufsberatung III. Wirkung - Nutzen - Sinn. Bielefeld: Bertelsmann, 15-29.

Hellbusch, J. (2019): Webseiten sind keine Anwendungen oder: Wie Sie Screenreadernutzer so richtig aus dem Tritt bringen. Online unter: https://www.hellbusch.de/webseiten-sind-keineanwendungen/\#more-2530. (Abrufdatum: 14.11.2019).

Klein, R. (2010): Beratung. In: R. Arnold, S. Nolda \& E. Nuissl (Hrsg.): Wörterbuch Erwachsenenbildung. 2. überarb. Auflage. Bad Heilbrunn: Julius Klinkhardt, S. 34-35.

Knab, M. (2014): In offenen Settings beraten. Ein Beitrag für eine gerechtere Teilhabe durch Bildungs- und Berufsberatung. In: I. Melter, E. Kanelutti-Chilas \& W. Stifter (Hrsg.): Zukunftsfeld Bildungs- und Berufsberatung III. Wirkung - Nutzen - Sinn. Bielefeld: Bertelsmann, 71-79.

Konter, A. (2019): Niedrigschwelligkeit. Socialnet Lexicon. Bonn: socialnet. Online unter: https:// www.socialnet.de/lexikon/Niedrigschwelligkeit. (Abrufdatum: 14.11.2019).

Mayrhofer, H. (2012): Niedrigschwelligkeit in der Sozialen Arbeit. Funktion und Formen aus soziologischer Perspektive. Wiesbaden: VS Verlag für Sozialwissenschaften.

Melter, I., Kanelutti-Chilas, E. \& Stifter, W. (Hrsg.) (2014): Zukunftsfeld Bildungs- und Berufsberatung III. Wirkung - Nutzen - Sinn. Bielefeld: Bertelsmann.

Menne, K. (2001) Jugendhilfeplanung für Erziehungs- und Familienberatung. Ergebnisse aus dem Modellprojekt im Landkreis Offenbach. Materialien zur Beratung, Bd. 9. Fürth: bke.

Nestmann, F., Engel, F. \& Sickendieck, U. (Hrsg.) (2014): Das Handbuch der Beratung. Bd. 2: Ansätze, Methoden und Felder. 3. Aufl. Tübingen: dgvt.

Reinkraut, R., Motulsky, S.L. \& Ritchie, J. (2009): Developing a Competent Practitioner: Use of Self in Counseling Psychology Training. Asian Journal of Counselling 16(1), 7-29.

Schürmann, I. (2014): Beratung in der Krisenintervention. In: F. Nestmann, F. Engel \& U. Sickendieck (Hrsg.): Das Handbuch der Beratung. Bd. 1: Disziplinen und Zugänge. 3. Aufl. Tübingen: dgvt, 523-534.

Schwarzer, C. \& Posse, N. (2014): Pädagogische Psychologie und Beratung. In: F. Nestmann, F. Engel \& U. Sickendieck (Hrsg.): Das Handbuch der Beratung. Bd. 1: Disziplinen und Zugänge. 3. Aufl. Tübingen: dgvt, 73-87.

Skiba, I. \& Züger, R. (2017): Basics Barrierefrei Planen. 2. Aufl. Basel: Birkhäuser.

Schweitzer, J. (2018): Vorwort. In: M. Weinhardt: Kompetenzorientiert systemisch beraten lernen. Aus der Reihe: Leben - Lieben - Arbeiten - Systemisch beraten. Göttingen: Vandenhoeck und Ruprecht, 9-13.

Tiefel, S. (2004): Beratung und Reflexion. Eine qualitative Studie zu professionellem Beratungshandeln in der Moderne. Wiesbaden: VS Verlag für Sozialwissenschaften.

Verein Deutscher Ingenieure - VDI (Hrsg.) (2011): VDI 6000 Blatt 3: Ausstattung von und mit Sanitärräumen - Versammlungsstätten und Versammlungsräume. Ohne Ort: Eigenverlag.

Wüllenweber, E. (2009): Krisen und Behinderung - Entwicklung einer praxisbezogenen Theorie zum Verstehen von Krisen und eines Handlungskonzeptes für die Krisenintervention bei Menschen mit geistiger Behinderung und bei Autismus. 3. Aufl. Hamburg: 53 Nord Agentur und Verlag. 


\section{Tanja Freifrau Schenck zu Schweinsberg (geb. Lück) und Dominik Rupprecht}

\section{Barrierefreie dialogorientierte Teilhabeplanung am Beispiel des Integrierten Teilhabeplans (ITP)}

Interdisziplinäre Erfahrungen bei der Entwicklung von inklusionsfördernden, interaktiven Erklärelementen in barrierefreien elektronischen Formularen - inBEF unter Beteiligung von Nutzer*innen

\section{Ausgangslage}

\subsection{Zielsetzung und Forschungsfrage}

Im Rahmen des Förderprogrammes „Forschung an Fachhochschulen mit Unternehmen (FHprofUnt)" wurde durch das Bundesministerium für Bildung und Forschung das Projekt "Inklusionsfördernde, interaktive Erklärelemente in barrierefreien E-Formularen (inBEF) “gefördert. Am Beispiel des Formulars Integrierter Teilhabeplan (ITP) sollten barrierefreie E-Formulare auf der Basis interaktiver Erklärelemente erforscht werden. Damit sollte ein Beitrag zur Inklusion von Menschen mit kognitiven Beeinträchtigungen geleistet werden. Es handelt sich hierbei um ein Instrument der Hilfeplanung, welches vom Institut Personenzentrierte Hilfen (IPH) GmbH zur Ermittlung von Teilhabebedarfen von Menschen mit Beeinträchtigungen konzipiert wurde (vgl. Gromann 2018). Entsprechend dem in der UN-Behindertenrechtskonvention (UN-BRK) verankerten gesellschaftspolitischen Leitgedanken der Chancengleichheit von Menschen mit Behinderungen und ihrer gleichberechtigten Teilhabe am Leben in der Gemeinschaft (vgl. BGBl. II 2008) nahm sich das interdisziplinäre Team des Projekts „inBEF“ der Problematik an, dass bei der Bearbeitung von Formularen Barrieren auftreten, die ein eigenständiges Ausfüllen verhindern sowie die Zugänglichkeit von Formularinhalten erschweren. Damit Menschen mit Behinderungen sich an den für sie installierten Teilhabeplanungen eigenständiger beteiligen können, um u.a. ihre aktuelle Situation, ihre Lebensziele sowie individuelle Hilfe- und Unterstützungsbedarfe zu benennen, sollte die barrierearme Software „meinITP“ entwickelt werden (vgl. Lück \& Gromann 2016). Das interdisziplinäre Forschungsteam der Fachbereiche Angewandte Informatik und Soziale Arbeit an der Hochschule Fulda - University of Applied Sciences - setzte sich unter Beteiligung von 
Nutzer*innen in den Forschungs- und Entwicklungsprozessen mit folgenden Forschungsfragen auseinander:

- Wie und in welcher Variation sind Erklärelemente zu gestalten, damit sie die Zugänglichkeit zum Formular und den damit verbundenen Inhalten für Nutzer*innen steigern?

- Was sind förderliche Faktoren und was sind hinderliche Faktoren aus Perspektive der Nutzer*innen beim Umgang mit dem Formular und wie können diese optimiert werden?

- Welche Wirkung hat der Einsatz interaktiver Erklärelemente auf die Kommunikations-, Interaktions- und Partizipationsprozesse für Nutzer*innen im Kontext von Integrierter Teilhabeplanung? (vgl. Lück \& Gromann 2016)

Des Weiteren waren technische Fragestellungen aus der modellbasierten Entwicklung von Software-Anwendungen Gegenstand des Forschungsinteresses. Sie fokussierten die automatische Generierung von barrierearmen elektronischen Formularen (vgl. Rupprecht u.a. 2015).

\subsection{Projektkonsortium}

Das interdisziplinäre Projektkonsortium bestand dabei zum einen aus Wissenschaftler*innen aus den Fachbereichen Sozialwesen und Angewandte Informatik der Hochschule Fulda - University of Applied Sciences - und dem Kompetenzzentrum für Mensch-Computer-Interaktion (KMCI). Zum anderen steuerte das Institut Personenzentrierte Hilfen (IPH) $\mathrm{GmbH}$ das bereits genannte PDF-basierte Instrument ITP als Arbeitsgrundlage bei, das im Vorhaben als exemplarischer Anwendungsbereich zum Einsatz kam. Hinzu kamen Praxispartner*innen aus dem Bereich der Behindertenhilfe. So konnte in Bezug auf den Einsatz des ITPs auf die Praxiserfahrungen der EVIM Gemeinnützige Behindertenhilfe $\mathrm{GmbH}$ und des Caritasverbandes für die Diözese Fulda e.V. Behindertenhilfe zurückgegriffen werden.

Die einzelnen Projektpartner*innen haben sich mit ihren jeweiligen Kompetenzen in das Projekt eingebracht. Die Hochschule Fulda hat neben der organisatorischen Projektleitung maßgeblich die Anforderungsanalyse, Realisierung und Evaluation durchgeführt. Die IPH GmbH gab detaillierte Einblicke in den aktuellen Ist-Zustand des Ausfüllprozesses für ITP-Formulare und definierte unter Beteiligung der anderen Projektpartner*innen den gewünschten Soll-Zustand für die jeweiligen Folge-Iterationen. Die beiden Praxispartner*innen haben kontinuierlich Nutzer*innen begleitet, die nach den jeweiligen Evaluationsphasen die entwickelten Prototypen weiter im Alltag genutzt bzw. erprobt haben. Sie beteiligten sich zudem an der Definition des zu erreichenden Soll-Zustands und gaben Rückmeldungen zu den erstellten Prototypen. 


\subsection{Zielgruppe}

Die Entwicklung und Erprobung einer softwarebasierten „meinITP“-Version wurde mit 14 Nutzer*innen aus zwei Einrichtungen der Behindertenhilfe und fünf Vertreter*innen der Selbsthilfe - hauptsächlich mit psychischen Beeinträchtigungen - umgesetzt. Sie hatten die Gelegenheit, in mehreren Erprobungsterminen das ITP-Dokument mit verschiedenen softwarebasierten Benutzeroberflächen zu bearbeiten. Ihre Erfahrungen in der Anwendung und Hinweise zur Zugänglichkeit flossen in die technische Weiterentwicklung der Prototypen ein. Beteiligt waren sieben Frauen und sieben Männer, die unterschiedliche Wohnund/oder Arbeitsangebote der Behindertenhilfe in ambulanter oder stationärer Form erhielten (vgl. Lück \& Gromann 2016, 42ff.).

Die zunächst überwiegend schriftbasierte Kommunikation im Formular stellte für alle Nutzer*innen mit kognitiven Beeinträchtigungen eine Barriere dar. Zu klären war, mit welchen alternativen Repräsentationsformen die Inhalte besser verstehbar und somit für die Zielgruppe handhabbar wurden. Eine Vielfalt an Nutzungsbarrieren wurde von den beteiligten Personen erkannt und benannt. Jede Person brachte ihre Sichtweise und individuelle Möglichkeiten in die Explorations- und Erprobungsphasen ein. Bei Bedarf wurde den Nutzer*innen eine persönliche Assistenz zur Seite gestellt, so dass bei Fragen zur Anwendung oder auftretenden Schwierigkeiten im Erprobungsprozess Unterstützung eingefordert werden konnte (vgl. ebd.).

\section{Grundlagen}

\subsection{Ansatz der Unterstützten Kommunikation}

Unterstützte Kommunikation bietet vielfältige alternative Kommunikationsmöglichkeiten, um Menschen mit angeborenen oder erworbenen Beeinträchtigungen jeden Alters eine (bessere) Verständigung zu ermöglichen. Durch den Einsatz ergänzender und/oder ersetzender Kommunikationsalternativen wie: Gegenstände, grafische Symbole, Gebärden, technisch-gestützte Kommunikationshilfen sollen die Beteiligungsmöglichkeiten und die Mitbestimmung der Betroffenen verbessert werden. Die Einsatzmöglichkeiten dieser nichtelektronischen und/oder elektronischen Kommunikationshilfen sind vielfältig. Ausgehend von den individuellen Kommunikationskompetenzen des Einzelnen und den Bedarfen sind sie gemeinsam mit der Person und dem jeweiligen Bezugssystem zu planen und in den Alltag zu transferieren (vgl. Kristen 2005; Braun 2014).

Die Beteiligung Betroffener an der Planung und der Umsetzung ihrer Teilhabeplanung ist nach Lück \& Gromann $(2016,43)$ Gegenstand eines personenzentrierten Ansatzes. Unterstützte Kommunikation greift hier, um die Inhalte dieses Prozesses für Menschen mit kognitiven Beeinträchtigungen zugänglicher zu machen. Im 
Kern geht es darum, „das Recht auf Partizipation und Beteiligung im Kontext der sozialen Rechte - hier der Eingliederungshilfe - wirklich barrierearm zu praktizieren und erst bei Bedarf Unterstützung zur Bearbeitung von Dokumentenfeldern durch professionell Helfende einzuholen“" (Lück \& Gromann 2016, 43).

Unter Bezugnahme auf Artikel 2 der UN-BRK ist festzuhalten, dass dort auf sämtliche Kommunikationsalternativen der Unterstützten Kommunikation verwiesen wird als zu schaffende Voraussetzung, damit Menschen mit Beeinträchtigungen an der gleichberechtigten Teilhabe in unserer Gesellschaft nicht behindert werden (vgl. Seiler-Kesselheim \& Wachsmuth 2014).

„Im Sinne dieses Übereinkommens schließt ,Kommunikation' Sprachen, Textdarstellung, Brailleschrift, taktile Kommunikation, Großdruck, leicht zugängliches Multimedia, sowie schriftliche, auditive, in einfache Sprache übersetzte, durch Vorleser zugänglich gemachte sowie ergänzende und alternative Formen, Mittel und Formate der Kommunikation, einschließlich leicht zugänglicher Informations- und Kommunikationstechnologie, ein" (Artikel 2 Abs. 1 UN-BRK).

Um eine bessere Zugänglichkeit zu Dokumenten zu schaffen, wurden im interdisziplinären Projekt „inBEF“ exemplarisch im Hilfeplaninstrument ITP Mittel der Unterstützten Kommunikation stufenweise implementiert und spezifische Erklärelemente in Zusammenarbeit mit Nutzer*innen entwickelt.

\subsection{Begriff Erklärelemente}

Die Entwicklung von Erklärelementen und die Art der Ausgestaltung und Positionierung in der „meinITP“-Anwendung waren von zentraler Bedeutung. Erklärelemente sind alternative Repräsentationsformen zur Verdeutlichung von schriftbasierten Dokumenteninhalten. Sie ermöglichen ein besseres Verstehen und Bearbeiten des Formulars. Sie wurden in verschiedenen Varianten entwickelt und durch Nutzer*innen überprüft. Dadurch können verschiedene Ebenen der Informationsvermittlung gewährleistet werden. Dies ermöglicht einen besseren Zugang zum Formular und eine eigenständigere Bearbeitung durch Menschen mit kognitiven Beeinträchtigungen. In den Untersuchungen wurden folgende statische und dynamische Varianten der Erklärelemente verwendet:

- eigene Fotos

- grafische Symbole

- auditive Erklärelemente

- avatarbasierte Erklärelemente

- einfache Sprache

- Leichte Sprache

Die Anordnung dieser Erklärelemente wurde auf Basis von Rückmeldungen der Nutzer*innen vollzogen (vgl. Lück \& Gromann 2016, 45ff.). 


\subsection{Benutzerzentrierte Entwicklung}

In einer benutzerzentrierten Entwicklung angelehnt an DIN EN ISO 9241-210 (ISO 9241-210, 2010) wird in mehreren Iterationen ein vorgegebener Entwurfsprozess durchlaufen, um Systeme gebrauchstauglich und zweckdienlich zu machen. Dazu soll sich ein solcher Gestaltungsprozess auf die Nutzer*innen, deren Erfordernisse und Anforderungen konzentrieren sowie Techniken und Kenntnisse der Arbeitswissenschaften auf dem Gebiet der Gebrauchstauglichkeit anwenden (vgl. ISO 9241-210, 2010, 4). Zu Beginn steht die Planung des benutzerzentrierten Prozesses in Bezug auf das neu zu entwickelnde System. Es folgt ein Kreislauf mit den Phasen Nutzungskontext verstehen und beschreiben, Nutzeranforderungen spezifizieren, Gestaltungslösungen entwickeln und die Evaluation aus der Nutzerperspektive.

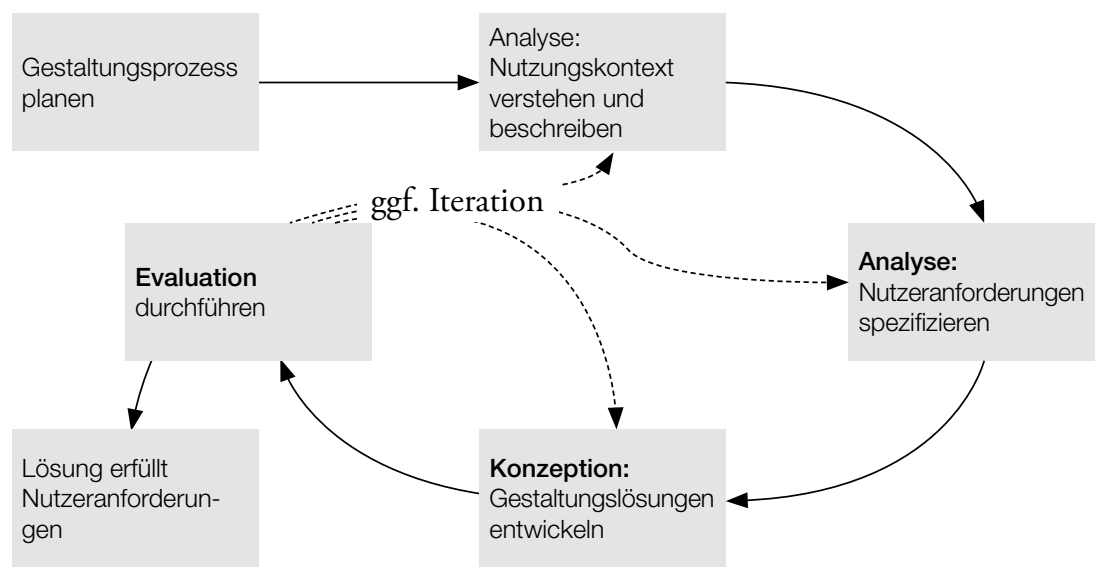

Abb. 1: Kreislauf einer benutzerzentrierten Entwicklung (eigene Anfertigung nach DIN EN ISO 9241-210)

Dieser Kreislauf wird solange durchlaufen, wie die Evaluationsergebnisse einen Bedarf hierfür aufzeigen. Dabei können nach jeder Evaluation auch einzelne Phasen ausgelassen und der Kreislauf verkürzt werden. Dieser Kreislauf hat sich bei der Erstellung interaktiver Systeme als Standard durchgesetzt, da er die Kommunikation zwischen den verschiedenen Stakeholdern wie Projektleiter*innen, Usability-Ingenieur*innen und Nutzer*innen dahingehend unterstützt, dass eine konsensfähige Grundlage für das Planen und Festlegen von Usability-Engineering Maßnahmen vorliegt. Aufgrund der hohen Einbeziehung von Nutzer*innen, ihren Erfordernissen und Anforderungen werden Hindernisse, die durch die Benutzung entstehen können, reduziert und das Risiko verringert, dass das System von den Nutzer*innen zurückgewiesen wird. 


\section{Vorgehen}

\subsection{Anforderungsermittlung der Formulargestaltung}

Das Projekt folgte der zuvor dargestellten benutzerzentrierten Entwicklungsmethodik. Zu Projektstart wurde eine Anforderungsermittlung und Nutzungskontextanalyse unter Beteiligung aller Projektmitglieder durchgeführt. Ein erster Ansatz bestand darin, Personas, also prototypische Benutzer*innen der Anwendung, zu erstellen, mit deren Hilfe zu Beginn ohne direkte Beteiligung von Nutzer*innen die Evaluation durchgeführt werden sollte. In der Arbeit mit den Nutzer*innen stellte sich jedoch heraus, dass eine frühere Einbindung von Nutzer*innen als geplant möglich war. Die Nutzer*innen waren sehr motiviert, an der Weiterentwicklung des ursprünglichen ITP-Formulars zu einer interaktiven Softwareanwendung etwas beitragen zu können.

Die erste Iteration der Anforderungsermittlung ergab zudem, dass sich das zugrundeliegende ITP-Formular in zwei grundlegende Bereiche einteilen ließ: In Kern-und Hilfsprozesse. Mit Hilfe des IPHs wurde jeder Bereich des ITPs für die Umsetzung in ein interaktives „meinITP“-Formular in diese beiden Bereiche eingeteilt. Zu den Kernprozessen gehörte demnach die eigene, aktuelle Situation der Nutzer*innen, die Definition eigener Ziele, die genauere Beschreibung der eigenen Fähigkeiten und Beeinträchtigungen, ein Überblick über die aktuelle Arbeitssituation und abschließend die Planung des zukünftigen Vorgehens. Über allem steht dabei immer das eigene Lebensziel der Nutzer*innen, auf das sich alle weiteren Angaben beziehen. $\mathrm{Zu}$ den Hilfsprozessen gehören Abschnitte wie die abschließende Unterschrift unter dem Formular oder personenbezogene Daten, die für den reinen Planungsprozess eher nebensächlich sind. Aus dieser Aufteilung wurde die grundlegende Navigations- und Grundstruktur der „meinITP“-Anwendung abgeleitet.

Ein weiterer Ansatz zur Erfassung von Anforderungen war die Verwendung vorhandener Richtlinien in Bezug auf die barrierefreie Gestaltung von Anwendungen. Typische Richtlinien wie das Vermeiden von Scrollbars, einstellbare Schriftgröße oder die Verwendung von kurzen und einfachen Sätzen und der einfachen Sprache, wurden angewandt. Diese Richtlinien halfen auch in der Diskussion über neue Funktionalitäten und deren Umsetzung in der Anwendung, da man mit ihnen eine Grundlage für eine erste Evaluation zur Hand hatte.

\subsection{Prototypen der Benutzungsoberfläche}

$\mathrm{Zu}$ Projektbeginn wurden drei Evaluationsiterationen der zu entwickelnden Benutzeroberfläche für die „meinITP“-Software geplant. Allerdings wurde dann in kürzeren Zyklen als den ursprünglich geplanten drei Zyklen iteriert. Die einzelnen Zwischenschritte der Oberflächenentwicklung wurden mittels mehrerer Prototypen in unterschiedlichsten Arten evaluiert und im nächsten Zyklus angepasst. Die notwendigen Änderungen reichten von minimalen Anpassungen, wie 
der Neuplatzierung von Oberflächenelementen, bis hin zu einer grundlegenden Überarbeitung des gesamten Designs. Das aktuelle Design entspricht den im Projekt ermittelten Anforderungen. Dabei hat sich der Einsatz von Rapid Prototyping als gewinnbringend herausgestellt. Zum einen konnten in kurzer Zeit verschiedene Layout-Varianten getestet und gegeneinander abgewogen werden. Zum anderen ermöglichte es die Anschaulichkeit der Prototypen im Dialog mit den Projektpartner*innen, neue Vorschläge für Funktionalitäten zügig auf ihre Brauchbarkeit zu prüfen. Das heißt es wurden u.a. Papierprototypen der Anwendung mit ggf. neuen Funktionen erstellt. Die Auswirkung auf andere Teile der Anwendung war gut ersichtlich und konnte somit diskutiert werden. Den Abschluss bildete dann eine HTML-Umsetzung. Die prototypische Realisierung mittels Web-Technologien bietet den Vorteil, dass die „meinITP“-Software sowohl lokal auf einzelnen PCs als auch in einem Intranet einer Einrichtung oder auch über das Internet (z.B. bundesweit) betrieben werden kann. Die „meinITP“-Software kann damit in verschiedenen Konstellationen erprobt und installiert werden, womit diverse Nutzungssituationen abgedeckt werden können.

\subsection{Entwicklung und Erprobung von Erklärelementen}

Nachdem die Navigations- und Grundstruktur der Anwendung sowie die Anordnung von Feldern auf der Bildschirmoberfläche erprobt und bestimmt war, widmete sich das interdisziplinäre Forschungsteam in enger Zusammenarbeit mit Nutzer*innen der Entwicklung von Erklärelementen. Es wurden in einem ersten Erprobungsabschnitt statische Erklärelemente in Form von grafischen Symbolen außerhalb des softwarebasierten Prototypens von Nutzer*innen gesichtet und auf Verständlichkeit geprüft. Mit Hilfe konkreter Papiermaterialien wurden die grafischen Symbole im Rahmen eines Workshops von ihnen bewertet. Die für hilfreich erachteten statischen Erklärelemente wurden im Anschluss in die Navigations- und Grundstruktur des Programmes implementiert. Es schloss sich eine erneute Überprüfung der implementierten statischen Erklärelemente an und weitere statische Varianten sowie neue auditive Erklärelemente wurden in das Programm aufgenommen. Durch eine Vorlesefunktion konnten Inhalte nun auch auditiv erfahrbar gemacht werden. Hier waren Elemente der Unterstützten Kommunikation von zentraler Bedeutung: Grafische Symbole, die Möglichkeit der Implementierung eigener Fotos sowie auditive Erklärelemente kamen zunächst zum Einsatz.

Ergebnis der sich anschließenden Entwicklungs- und Erprobungsphasen sind handlungsorientierte 3D-Szenen mit Avataren: Sie veranschaulichen Items aus dem Integrierten Teilhabenplan, basierend auf der Internationalen Klassifikation der Funktionsfähigkeit, Behinderung und Gesundheit (ICF: International Classification of Functioning, Disability and Health) der Weltgesundheitsorganisation (WHO), Komponente d: Aktivitäten und Partizipation (vgl. DIMDI 2005), um Menschen mit Beeinträchtigungen die Möglichkeit zur Selbsteinschätzung ihrer Situation zu ge- 
ben (vgl. Lück \& Gromann 2016, 44ff.). Die hier abgefragten Fähigkeiten sollen Nutzer*innen auf einer Skala von 1 für „keine Beeinträchtigung“ bis 5 für „,voll ausgeprägte Beeinträchtigung “ bewerten. Im Projekt galt es, den Klienten*innen die Semantik solcher handlungsorientierten Prozesse mit ihren jeweiligen Abstufungen über adäquate Visualisierungen zu vermitteln. Hierzu wurden für jedes umgesetzte ICF-Item fünf Drehbücher erstellt, die die unterschiedlichen Ausprägungen der Skala visualisieren. Ausgehend von diesen Drehbüchern entstanden Avatar-basierte 3D-Szenen (eine bis mehrere Szenen pro ICF-Skala). Dabei sind pro ICF-Skala Avatar-Animationen von 15 Sekunden bis zu einer Minute entstanden.
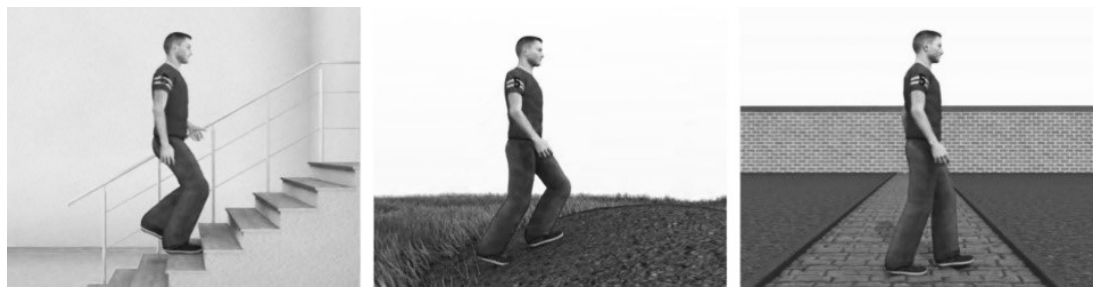

Abb. 2: Screenshots aus der Szene „sich in verschiedenen Umgebungen fortbewegen“

Abbildung 2 zeigt exemplarisch drei Screenshots aus der Animation für das ICFItem ,sich in verschiedenen Umgebungen fortbewegen“. In der dargestellten Szene, die die Bewertungsstufe „keine Beeinträchtigung“ darstellt, wird gezeigt, dass die Person ohne Probleme die drei abgebildeten Situationen meistern kann. Für die Bewertungsstufe „voll ausgeprägte Beeinträchtigung“ hingegen steht die Person vor den drei Hindernissen (Treppe, Hügel, Kopfsteinpflaster), schüttelt den Kopf und ist traurig, dass sie sie nicht überwinden kann. Für die Bewertungsstufen dazwischen werden unterschiedliche Schwierigkeiten für die Person gezeigt etwa, dass sie auf der Treppe mehrmals anhalten muss, da ihr die Ausdauer fehlt, oder aber, dass sie auf der Straße mit Kopfsteinpflaster stolpert.

Aus Sicht der Nutzer*innen werden die aufgeführten Erklärelemente mittels einer Erklärbox eingefügt. Sie ermöglicht es, individuelle Formen der Informationsvermittlung eigenständig zu aktivieren. Die Funktion meineBILDER ermöglicht, dass einzelne Symbolfelder durch eigene Fotos individuell anpassbar sind und dadurch das Formular für einzelne Nutzer*innen verständlicher wird (vgl. Lück \& Gromann 2016, 45ff.).

\section{Dialogorientierte Erprobungsabschnitte mit Nutzer*innen}

\subsection{Methodik und Settings}

Die Beteiligung von Menschen mit Beeinträchtigungen in die Entwicklungs- und Erprobungsabschnitte ist zentrales Element des „inBEF-Projektes“. Im Dialog mit 
ihnen wurde die Umsetzung praktiziert. Es wurde eine wissenschaftliche Mitarbeiterin des Fachbereichs Sozialwesen mit Kompetenzen in der Anwendung Unterstützter Kommunikation zur Durchführung dialogorientierter Erprobungsabschnitte mit Nutzer*innen beauftragt. Die Erkenntnisse aus den verschiedenen Erprobungsabschnitten wurden von ihr an die Projektmitarbeitenden des Fachbereichs Angewandte Informatik zurückgekoppelt. Daraus wurden nächste technische Umsetzungsschritte abgeleitet, die eine Weiterentwicklung des Prototypens zur Folge hatte. Die neue Version konnten Nutzer*innen dann erneut erproben und Angaben hinsichtlich der Nutzbarkeit machen. Diese wurden evaluiert und flossen wiederum in die Programmentwicklung ein. Insgesamt wurden sieben Erprobungszyklen durchgeführt. Diese Erprobungszyklen fanden in unterschiedlichen Settings und an verschiedenen Orten statt. Zu Beginn wurden Erprobungen des Prototypens in Einzelgesprächen am Computer durchgeführt. Hier wurde die vorhandene Navigations- und Grundstruktur des Prototypens von Nutzer*innen ausprobiert und bewertet. Die Exploration des Prototypens im Einzel-Setting am Computer erfolgte vor Ort im Wohnheim oder am Arbeitsplatz der Nutzer*innen und wurde von der Projektmitarbeiterin des Fachbereichs Sozialwesen durchgeführt. Für Rückfragen standen Assistent*innen der Nutzer*innen aus den Einrichtungen zur Verfügung (vgl. Lück \& Gromann 2016, 43ff.).

Der Exploration am Computer schloss sich ein leitfadengestütztes Einzelinterview an, um förderliche und hinderliche Faktoren aus Sicht der Nutzer*innen zu ermitteln. Ein weiteres Setting war die Durchführung eines Workshops an der Hochschule Fulda zur Erprobung statischer Erklärelemente außerhalb des Prototypens. Verschiedene grafische Symbole auf Papiermaterial wurden von Nutzer*innen gesichtet und sie konnten auswählen, welche dieser grafischen Symbole das Verstehen von Programminhalten befördern. Anhand der verschiedenen Rückmeldungen von Nutzer*innen aus den diversen Settings erfolgten die Anpassungen des Prototyps wie z.B. die Überarbeitung vorhandener und Implementierung neuer Erklärelemente (vgl. ebd.).

Für die Durchführung dieses personenzentrierten und iterativen Entwicklungsprozesses war eine feste Ansprechpartnerin aus der Gruppe der Wissenschaftler*innen für die Nutzer*innen wichtig. Ihr nicht direktives, sondern begleitendes und unterstützendes Handeln trug zum Gelingen der dialogorientierten Erprobungsabschnitte mit Nutzer*innen bei. Die Anwendung von Elementen Unterstützter Kommunikation, einfacher Sprache sowie Leichter Sprache, orientiert an den Empfehlungen des „Netzwerkes Leichte Sprache“ (vgl. Netzwerk Leichte Sprache e.V. 2019) waren methodisch signifikant. Einzelne Erprobungs-Settings wurden gefilmt, um Aufschluss über das Gelingen von Kommunikations- und Interaktionsprozessen der Nutzer*innen zu erlangen als auch Aspekte der Usability (Benutzerfreundlichkeit, Gebrauchstauglichkeit) durch quantitativ-technische Analyse unter Berücksichtigung vorhandener Richtlinien zu identifizieren (vgl. Lück \& Gromann 2016, 43ff.). 


\subsection{Nutzerbasierte Erprobungsabschnitte}

Tab. 1: Erprobungsabschnitte des Projektes „inBEF“ in zeitlicher Reihenfolge (modifiziert und angelehnt an Lück \& Gromann 2016, 46)

\begin{tabular}{|c|c|c|}
\hline \multicolumn{3}{|c|}{ „inBEF“ Erprobungsabschnitte Mai 2013 bis März 2015} \\
\hline Monat & Inhaltlicher Fokus der Erprobung & Setting \\
\hline Mai 2013 & Navigations- und Grundstruktur des Prototyps & $\begin{array}{l}\text { Einzel-Setting } \\
\text { am Computer }\end{array}$ \\
\hline Oktober 2013 & $\begin{array}{l}\text { statische Erklärelemente auf Papier außerhalb des } \\
\text { Prototyps }\end{array}$ & Workshop \\
\hline März 2014 & $\begin{array}{l}\text { erneuerte Navigations- und Grundstruktur inklusive } \\
\text { implementierter statischer Erklärelemente }\end{array}$ & $\begin{array}{l}\text { Einzel-Setting } \\
\text { am Computer }\end{array}$ \\
\hline Mai 2014 & $\begin{array}{l}\text { statische und auditive Erklärelemente implementiert } \\
\text { im Prototyp }\end{array}$ & $\begin{array}{l}\text { Einzel-Setting } \\
\text { am Computer }\end{array}$ \\
\hline Juli 2014 & $\begin{array}{l}\text { erneuerte Navigations- und Grundstruktur: avatarba- } \\
\text { sierte Erklärelemente zu „Fähigkeiten oder Beeinträch- } \\
\text { tigungen der Teilhabe in Selbstsorge/Wohnen, [...] } \\
\text { Freizeit/Teilhabe an Gesellschaft“ exemplarisch mit } \\
\text { ICF-bezogenem Item „Essen“ }\end{array}$ & $\begin{array}{l}\text { Einzel-Setting } \\
\text { am Computer }\end{array}$ \\
\hline $\begin{array}{l}\text { September } \\
2014\end{array}$ & $\begin{array}{l}\text { erneuerte Navigations- und Grundstruktur: avatarba- } \\
\text { sierte Erklärelemente zu „Fähigkeiten oder Beeinträch- } \\
\text { tigungen der Teilhabe in Selbstsorge/Wohnen, [...] } \\
\text { Freizeit/Teilhabe an Gesellschaft“ exemplarisch mit } \\
\text { ICF-bezogenem Item „Essen“ in weiteren Abstufungen }\end{array}$ & $\begin{array}{l}\text { Einzel-Setting } \\
\text { am Computer }\end{array}$ \\
\hline März 2015 & $\begin{array}{l}\text { avatarbasierte Erklärelemente zu „Fähigkeiten oder Be- } \\
\text { einträchtigungen der Teilhabe in Selbstsorge/Wohnen, } \\
\text { [...] Freizeit/Teilhabe an Gesellschaft“ exemplarisch } \\
\text { mit ICF-bezogenen Items „Essen“, „Einkaufen“, „sich } \\
\text { in verschiedenen Umgebungen fortbewegen“ in weite- } \\
\text { ren Abstufungen }\end{array}$ & $\begin{array}{l}\text { Einzel-Setting } \\
\text { am Computer }\end{array}$ \\
\hline
\end{tabular}

\section{Ergebnisse und Diskussion}

In der Entwicklung der interaktiven „meinITP“-Anwendungen haben sich zusammenfassend folgende positive und förderliche Faktoren ergeben: Der Umgang mit dem komplexen, ursprünglichen ITP-Formular konnte durch eine Neusegmentierung in Kern- und Hilfsprozesse für die Nutzer*innen verständlicher gestaltet werden. Diese Einteilung ermöglichte ihnen zudem eine einfache Navigation innerhalb der Dokumentenstruktur. Durch die Überführung des ITPs in 
ein interaktives, elektronisches Formular ist es gelungen, die durch Nutzer*innen getätigten Formulareingaben automatisch in ein sozialadministrativ nutzbares PDF-Dokument zu überführen.

Der Einsatz von Prototypen ermöglichte häufigere Evaluationen, auch von Zwischenschritten, in der Entwicklung von Erklärelementen und Benutzeroberfläche. Dabei hat die frühzeitige Einbeziehung der Nutzer*innen in die Entwicklungsund Erprobungsphasen von Prototypen den Forschungsprozess bereichert. Durch die dargestellte Arbeitsweise im Projektteam wurde somit ein interdisziplinäres Vorgehen gefördert. So konnten Forscher*innen aus den Bereichen Angewandte Informatik und Soziale Arbeit mit Nutzer*innen reibungslos zusammenarbeiten und die Entwicklung stetig vorantreiben.

Eine feste Ansprechpartnerin aus dem interdisziplinären Forschungsteam mit Kompetenzen in der Anwendung Unterstützter Kommunikation für Nutzer*innen war wichtig. Die Anwendung computergestützter Formularbearbeitung wurde von Nutzer*innen mit hoher Motivation und Begeisterung umgesetzt und hat bewirkt, dass ein barrierearmer Prototyp der Software „meinITP“ vorliegt. Menschen mit geringer Schreib- und Lesekompetenz können erste Teile des Integrierten Teilhabeplans bearbeiten. Dafür ist die umgesetzte Anreicherung mit Erklärelementen förderlich. Das zuvor rein auf Schriftsprache ausgelegte Formular beinhaltet jetzt verschiedene Varianten von Erklärelementen: eigene Fotos, grafische Symbole, auditive und avatarbasierte Erklärelemente, einfache Sprache sowie Leichte Sprache. Erklärelemente bedürfen auch der individuellen Anpassbarkeit: die Implementierung von eigenen Fotos hilft Nutzer*innen beim Umgang mit dem Formular (vgl. Lück \& Gromann 2016, 47ff.). Erklärelemente sollten bei der Formulargestaltung in Multimodalitäten vorhanden sein, um eine barrierearme Teilhabeplanung praktizieren zu können. Ausgehend von der Beobachtung, dass gelingende Formularbearbeitung bei Teilen von Nutzer*innen gekoppelt ist an Assistenz, bedarf es einer reflektierten Prozessbegleitung seitens Assistent*innen, um die Meinungsbildung von Nutzer*innen zu erfassen. Denn Menschen mit Beeinträchtigungen sind oftmals sensibilisiert für die Erwartungshaltung von Fachpersonen und erwünschtem Verhalten.

\section{Literatur}

Braun, U. (2014): Was ist Unterstützte Kommunikation? In: ISAAC - Gesellschaft für Unterstützte Kommunikation e.V. (Hrsg.): Handbuch der Unterstützten Kommunikation. Band I, Grundwerk, 6. Auflage. Karlsruhe: von Loeper Literaturverlag im Ariadne Buchdienst, 01.003.001-01.005.001.

Deutsches Institut für Medizinische Dokumentation und Information (DIMDI) (Hrsg.) (2005): Internationale Klassifikation der Funktionsfähigkeit, Behinderung und Gesundheit der WHO. Klassifikation der Aktivitäten und Partizipation (Komponente d). Stand 19.06.2012. Online unter: https://www. dimdi.de/static/de/klassifikationen/icf/icfhtml2005/component-d.htm. (Abrufdatum: 09.12.2019). 
DIN EN ISO 9241-210 (2010). Ergonomie der Mensch-System-Interaktion Teil 210: Prozess zur Gestaltung gebrauchstauglicher interaktiver Systeme. Berlin: Beuth.

Gesetz zu dem Übereinkommen der Vereinten Nationen vom 13. Dezember 2006 über die Rechte von Menschen mit Behinderungen sowie zu dem Fakultativprotokoll vom 13. Dezember 2006 zum Übereinkommen der Vereinten Nationen über die Rechte von Menschen mit Behinderungen. Bundesgesetzblatt (BGBl.) Teil II, Nr. 35 1419-1457, ausgegeben zu Bonn am 31. Dezember 2008: Bundesanzeiger Verlag.

Gromann, P. (2008): Der Integrierte Teilhabeplan „ITP“ als Bedarfsermittlungsinstrument in mehreren Bundesländern. In: Projekt „Umsetzungsbegleitung Bundesteilhabegesetz“ Deutscher Verein für öffentliche und private Fürsorge e.V. Online unter: https://umsetzungsbegleitung-bthg.de/w/ files/links-und-downloads/gromann-einfuehrung-itp-in-mehreren-bundeslaendern.pdf. (Abrufdatum: 25.11.2019).

Kristen, U. (2005): Praxis Unterstützte Kommunikation. Eine Einführung. 5. Auflage. Düsseldorf: Verlag Selbstbestimmtes Leben.

Lück, T. \& Gromann, P. (2016): Teilhabepläne selbst erarbeiten - zur Entwicklung der barrierearmen Software „meinITP“. In: P. Gromann (Hrsg.). Teilhabeorientierte Steuerung sozialpsychiatrischer Hilfen. 1. Auflage. Bonn: Psychiatrie Verlag, 42-50.

Netzwerk Leichte Sprache e.V. (2019): Die Regeln für Leichte Sprache. Online unter: http://www. leichtesprache.com/dokumente/upload/21dba_regeln_fuer_leichte_sprache.pdf. (Abrufdatum: 27.11.2019).

Rupprecht, D., Etzold, J. \& Bomsdorf, B. (2015). „Model-based development of accessible, personalized web forms for ICF-based assessment". In: Proceedings of the 7th ACM SIGCHI Symposium on Engineering Interactive Computing Systems (EICS '15). ACM, New York, NY, USA, 120-125. DOI: https://doi.org/10.1145/2774225.2775077.

Seiler-Kesselheim, A. \& Wachsmuth, S. (2014): Die Bedeutung der UN-Konvention über die Rechte von Menschen mit Behinderungen für unterstützt Kommunizierende. In: von Loeper Literaturverlag und isaac - Gesellschaft für Unterstützte Kommunikation e.V. (Hrsg.): Handbuch der Unterstützten Kommunikation. Band I, Grundwerk, 6. Auflage. Karlsruhe: von Loeper Literaturverlag, 16.025.001-16.033.001. 
Barbara Klein

\section{Assistive und andere Technologien}

\section{Ausgangssituation}

Barrierefreie Umgebungen sind eine notwendige Voraussetzung für ein selbstständiges Leben, doch reichen diese bei verschiedenen Funktionsverlusten und/oder -einschränkungen häufig nicht aus. Ziel dieses Beitrages ist es, einen Überblick über Konzepte wie das Universal Design oder Assistive Technologien und Hilfsmittel zu geben und die in diesem Kontext häufig verwendeten Begrifflichkeiten zu erläutern und ihren Beitrag für ein selbstständiges und teilhabeorientiertes Leben aufzuzeigen. Die Vielfalt der technologischen Entwicklungen hat dabei Auswirkungen auf alle am Versorgungsprozess beteiligten Akteure. Die zunehmende technologische Komplexität erfordert neue Qualifikationsanforderungen, und weitere Akteure in den Versorgungsstrukturen. Dazu werden auch Transferkonzepte benötigt, um allen Akteursgruppen den Zugang zu Informationen zu ermöglichen, so dass diese sich ein umfassendes Bild zu den Möglichkeiten für ein selbstständiges Leben und Teilhabe machen können.

\subsection{Soziodemographische Daten zum potenziellen Bedarf Assistiver Technologien}

Deutschland gehört neben Japan und Italien zu den "Super-Aged Societies“. Das Kennzeichen von Super-Aged Societies ist, dass der Anteil der Menschen, die 65 Jahre und älter sind, mehr als $21 \%$ der Bevölkerung beträgt (vgl. AARP 2017, 2). Das Älterwerden ist dabei - auch bei gesunden Personen - in der Regel von alternsbedingten Funktionsverlusten der Sensorik, Mobilität und Kognition begleitet. Funktionseinschränkungen oder -verluste aufgrund von Behinderung, Krankheit oder Unfällen gibt es in jedem Lebensalter, doch zahlenmäßig besonders relevant werden auch diese im höheren Lebensalter. Der Anteil hochaltriger Menschen, also Personen ab einem Alter von ca. 80 Jahren, steigt in Deutschland seit langem kontinuierlich an. Gab es 2013 4,4 Millionen 80-jährige und ältere Menschen (vgl. Statistisches Bundesamt 2015, 21), so waren es nach der 14. koordinierten Bevölkerungsvorausberechnung 5,4 Millionen im Jahr 2018. Bis 2022 wird mit einem Anstieg auf 6,2 Millionen gerechnet und für 2050 werden zwischen 8,9 bis 10,5 Millionen erwartet (vgl. Statistisches Bundesamt 2019, 25).

Neben den alternsbedingten Funktionseinschränkungen steigt die Wahrscheinlichkeit, pflegebedürftig zu werden. Ende 2017 gab es 3,4 Millionen pflegebe- 
dürftige Menschen in Deutschland. Während bei den 65- bis 70-Jährigen die Wahrscheinlichkeit, pflegebedürftig zu werden, bei 3,8\% lag, scheint diese sich im Fünf-Jahresabstand fast zu verdoppeln: Sie lag bei den 80 - bis 85 -Jährigen bei 23,3\%, bei den 90-jährigen und älteren Menschen bei 70,7\% (vgl. Statistisches Bundesamt 2018a, 20). Im Jahr 2015 lebten laut Statistischem Bundesamt 7,8 Millionen schwerbehinderte Menschen ${ }^{1}$ in Deutschland; fast ein Drittel davon war über 75 Jahre und älter (vgl. Statistisches Bundesamt 2018b, 6). Pflegebedürftigkeit und Behinderung sind keine sich ausschließende Kategorien, vielmehr gibt es Überlappungen, so dass man von ca. 10 Millionen Menschen mit erheblichen Funktionseinbußen/-verlusten ausgehen kann. Dazu kommen alte und sehr alte Menschen, die weder einen Pflegegrad noch eine Behinderung haben, sondern alterungsbedingte Funktionseinbußen in der Sensorik, Mobilität und Kognition. Zieht man hier z.B. die Anzahl der schwerstbehinderten Menschen von der Gesamtaltersgruppe 75 Jahre und älter ab, würden allein in Deutschland rund 6,7 Millionen weitere Menschen dazu kommen, die einen Bedarf an barrierefreien Umgebungen und ergänzenden Assistiven Technologien haben.

\subsection{Assistive Technologien, Hilfsmittel und weitere Konzepte}

Für ein autonomes Leben bei Funktionseinschränkungen oder -verlusten kann der Einsatz von Assistiven Technologien dazu beitragen, diese auszugleichen oder zu kompensieren. Die Behindertenrechtskonvention der Vereinten Nationen (UN-BRK) fordert ein universelles Design von „Produkten, Umfeldern, Programmen und Dienstleistungen in der Weise, dass sie von allen Menschen möglichst weitgehend ohne eine Anpassung oder ein spezielles Design genutzt werden können“ (Art. 2 UN-BRK). Allerdings können - selbst bei einem universellen Design - für bestimmte Gruppen von Menschen Hilfsmittel erforderlich sein. Während die deutsche Rechtsprechung von Hilfsmitteln spricht, wird von der Weltgesundheitsorganisation (WHO) der Begriff „Assistive Technologien“ oder „Assistive Produkte“ verwendet (WHO 2004, 10).

Da es rund um den Begriff „Assistive Technologien“ eine Spannbreite ähnlicher Begrifflichkeiten und Konzepte gibt, werden diese im Folgenden kurz erläutert.

\section{Assistive Technologien/Hilfsmittel}

Die Weltgesundheitsorganisation (WHO) definiert Assistive Technologien als einen Oberbegriff für jedes Produkt/Gerät oder System, das es dem einzelnen ermöglicht, Aufgaben auszuführen, die ohne dieses entweder nicht ausgeübt werden können oder dadurch leichter und sicherer ausgeführt werden können (vgl. ebd.).

1 Menschen, denen von den Versorgungsämtern ein Grad der Behinderung (GdB) von 50 und mehr zuerkannt wurde, gelten als schwerbehindert ( $\$ 2$ Abs. 2 SGB IX). 
Unter Assistiven Technologien wird die Bandbreite der technischen Hilfsmittel und Hilfen zum täglichen Leben, Mobilitätshilfen sowie Sitz- und Lagerungshilfen, Umgebungssteuerung, Barrierefreiheit des Wohnumfelds und Arbeitsplatzanpassungen, Prothetik und Orthetik, sensorische Hilfen für Hörgeschädigte und Gehörlose und Unterstützte Kommunikation verstanden, aber auch organisatorische Konzepte wie Telecare und Telehealth werden unter diesem Begriff subsumiert (vgl. Connell u.a. 2008, 9).

In Deutschland wird anstelle des Begriffs Assistive Technologien vor allem der Begriff „Hilfsmittel“ in der relevanten Gesetzgebung und den dazugehörigen Verordnungen verwendet. Hilfsmittel sind eine verordnungsfähige Leistung der Kranken- und Pflegeversicherung und können auch auf anderen Gesetzesgrundlagen basieren. Die DIN EN ISO 9999 (vgl. DIN Institut für Normung e.V. 2017) beschreibt die Klassifikationen und Terminologie der Hilfsmittel für Menschen mit Behinderungen. Hier sind Hilfsmittel als ,jegliches Produkt (einschließlich Vorrichtungen, Ausrüstung, Instrumente und Software), Sonderanfertigung oder allgemeines Gebrauchsgut [definiert], das von oder für Menschen mit Behinderung verwendet wird

- für Teilhabe

- um Körperfunktionen/-strukturen und Aktivitäten zu schützen, zu unterstützen, zu ertüchtigen, zu messen oder zu ersetzen, oder

- um Schädigungen, Beeinträchtigungen der Aktivität oder Beeinträchtigungen der Teilhabe zu verhindern" (ebd., 6).

Innerhalb des Norm-Texts wird darauf verwiesen, dass die WHO-Initiative GATE (Global Cooperation on Assistive Health Technology) darauf hinweist, dass die Definition von Hilfsmittel erweitert werden muss, um sie mehr mit der Internationalen Klassifikation der Funktionsfähigkeit, Behinderung und Gesundheit (International Classification of Functioning, Disability and Health, ICF) in Einklang zu bringen. Des Weiteren sollte eine positivere Terminologie eingesetzt werden (vgl. ebd., 99). Die Hilfsmittel-Richtlinie (2018) orientiert sich in ihrer Definition lediglich am ersten Teil der Norm.

Nach $₫ 33$ Abs. 1 SGB V besteht für Krankenkassen eine gesetzliche Pflicht zur Hilfsmittelversorgung. Die Bewilligung eines Hilfsmittels setzt voraus, dass das Hilfsmittel

„im Einzelfall erforderlich ist, um den Erfolg der Krankenbehandlung zu sichern, einer drohenden Behinderung vorzubeugen oder eine Behinderung auszugleichen, soweit die Hilfsmittel nicht als allgemeine Gebrauchsgegenstände des täglichen Lebens anzusehen [...] sind“.

\subsection{Hilfsmittel}

Nach $\$ 139$ SGB V erstellt der Spitzenverband Bund der Krankenkassen ein Hilfsmittelverzeichnis, das regelmäßig aktualisiert und im Bundesanzeiger veröf- 
fentlicht wird. Das Hilfsmittelverzeichnis richtet sich vor allem an die verordnenden Berufe, u.a. wegen der für die Verordnung relevanten Hilfspositionsnummer, und an die Hersteller, da dort auch die Anforderungen der Produktuntergruppen beschrieben sind. Zurzeit umfasst das Hilfsmittelverzeichnis des GKV-Spitzenverbandes rund 32.500 Produkte, die in 41 Produktgruppen mit rund 800 Produktuntergruppen sowie ca. 2.600 Produktarten gegliedert sind (vgl. GKV Spitzenverband 2019, 15). Die momentanen technologischen Fortschritte, wie z.B. in der Sensorik, Robotik, Software etc., führen zu vielen Produktinnovationen, die ganz neue Möglichkeiten des Behinderungsausgleiches bieten. Berücksichtigt man dieses und die Anzahl der Hilfsmittel, wird deutlich, dass hier spezielle Expertise erforderlich ist, die bislang erst ansatzweise in der Ausbildung in den Gesundheitsberufen vermittelt wird und auch nicht flächendeckend bei den Dienstleistern (z.B. Sanitätshäusern) zur Verfügung steht.

Neben dem Hilfsmittelverzeichnis des GKV-Spitzenverbandes gibt es die Informationsplattform www.rehadat-hilfsmittel.de, die vom Institut der Deutschen Wirtschaft Köln e.V. betrieben und vom Bundesministerium für Arbeit und Soziales (BMAS) aus Mitteln des Ausgleichsfonds gefördert wird. Ziel dieser Plattform ist es, herstellerneutral zu Hilfsmitteln und technischen Arbeitshilfen in Deutschland zu informieren.

Die Gliederung der dort aufgeführten Produkte richtet sich nach den Lebensbereichen Arbeitsplatz \& Ausbildung, Mobilität \& Orientierung, Kommunikation \& Information, Bauen \& Wohnen, Haushalt \& Ernährung, Versorgung \& Hygiene, Therapie \& Training, Orthesen $\&$ Prothesen, Freizeit $\&$ Sport und enthält die ICF-bezogene Klassifizierung. Die Produkte können dabei im Hilfsmittelverzeichnis enthalten sein; viele sind dort jedoch nicht aufgeführt, da sie nicht unter die Leistungspflicht der GKV fallen.

Ein weiteres Internet-Portal ist wegweiseralterundtechnik.de, eine nationale Referenzdatenbank, die vom Ministerium für Arbeit und Sozialordnung, Familie, Frauen und Senioren Baden-Württemberg und dem Bundesministerium für Bildung und Forschung (BMBF) von 2011 bis 2014 gefördert wurde und über 200 Produkte rund um altersgerechte Assistenzsysteme beschreibt (allerdings mittlerweile nicht mehr aktualisiert zu werden scheint).

Die Internet-Plattform nullbarriere.de hat sich zum Ziel gesetzt, rund um Barrierefreiheit zu informieren und eine Vielfalt an Informationen regulativer Art, zu Produkten, Planungshilfen, Weiterbildung etc. zur Verfügung zu stellen. Monatlich kann diese Plattform fast 100.000 Besucher*innen verzeichnen - von betroffenen Menschen, Architekt*innen, Planer*innen und anderen Interessierten (vgl. https://nullbarriere.de/forum).

EASTIN ist ein weltweites Netzwerk, das über Hilfsmittel für Menschen mit Behinderungen informiert und aus Hilfsmitteldatenbanken vieler Länder gespeist wird. Über www.eastin.eu kann man sich zu rund 70.000 auf dem Markt ver- 
fügbaren Hilfsmitteln informieren und dazugehörige Informationen, Checklisten sowie praxisorientierte Hintergrundinformationen abrufen (vgl. www.eastin.eu/ de-de/GeneralInfo/List).

\subsection{Altersgerechte Assistenzsysteme und Smart Home-Technologien}

Der Begriff „Altersgerechte Assistenzsysteme“ ist die deutsche Entsprechung für „Ambient Assisted Living“ und stand lange Zeit im Kontext von Förderprogrammen der Europäischen Union und der Bundesregierung. Gemeint sind damit Konzepte, Produkte und Dienstleistungen, die neue Technologien in den Alltag einführen, um die Lebensqualität und Sicherheit für Menschen in allen Lebensphasen, vor allem im Alter, zu erhöhen (vgl. Ambient Assisted Living Deutschland 2016; Meyer 2018, 148).

Im Rahmen dieser Programme wurde eine Vielzahl von Projekten gefördert mit dem Ziel, neue sensorbasierte Produkte, wie z.B. Sturzerkennungsgeräte, zu entwickeln, die die Lebensqualität und Sicherheit älterer Menschen fördern sollen. Einige dieser Entwicklungen bewegen sich im Bereich von Smart Home und Sicherheitstechnologien. Hier gab es in den vergangenen Jahren im Consumer-Bereich einige Neuerungen und mittlerweile ein breites Angebot z.B. in den Elektrohandelsketten oder Discountern - ergänzt durch eine Palette an Fachzeitschriften rund um das smarte Zuhause.

Eine digitale Sprachassistenz hat in der Regel jedes Smartphone, dazu kommen seit kurzem interaktive Lautsprecher. Laut einer repräsentativen Befragung der Postbank (2019) nutzten im Jahr 2019 bereits $10 \%$ der befragten Personen eine Sprachassistenz.

Diese digitale Sprachassistenz kann mittels sog. „Skills“ zu vielen unterschiedlichen Funktionen befähigt werden, so dass z.B. über Spracheingabe das Telefon bedient und u.a. auch als Notruf genutzt werden kann (vgl. Stainhauser 2019). Diese Technologien eröffnen neue Optionen für ein Mehr an Sicherheit und Lebensqualität bei unterschiedlichen Funktionseinschränkungen mit dem Vor- und auch Nachteil, dass sie in der Regel nicht als klassisches Hilfsmittel gelten. Da diese Systeme Massenprodukte sind, werden sie nicht mit Alter und Behinderung assoziiert und passen sich in die „Normalität“ des Lebens ein. Von Nachteil könnte die bislang fehlende Kostenerstattung durch die Kranken- bzw. Pflegeversicherung sein.

\subsection{Robotik, Apps und andere Entwicklungen}

Wie bei den altersgerechten Assistenzsystemen gibt es rund um die Entwicklung robotischer Systeme viele interessante Forschungsprojekte. Erste Produkte sind kommerziell verfügbar. Da sind vor allem die Systeme zu nennen, die die Mobilität unterstützen, wie z.B. Exoskelette, die manchen Rollstuhlfahrer*innen zum Gehen verhelfen können, oder robotische Rollstühle und Trainingsgeräte. Mitt- 
lerweile gibt es zudem robotische Arme und Systeme, die die Nahrungsaufnahme unterstützen (vgl. Klein u.a. 2018, 87ff.).

Interessant sind auch Entwicklungen wie emotionale und soziale Roboter. Der Robbenroboter PARO wird z.B. in Deutschland in der stationären Altenhilfe überwiegend bei Menschen mit demenziellen Erkrankungen eingesetzt. Auch bei Menschen mit einem Wachkoma oder schwersten Behinderungen können diese Roboter eingesetzt werden (vgl. ebd., 62f.). Humanoide Roboter wie NAO oder PEPPER waren bis vor kurzem vor allem Forschungs- und Entwicklungsplattformen, um Schüler*innen und Studierende in Bezug auf Robotik zu qualifizieren. Mittlerweile werden Applikationen für den professionellen Einsatz im Sozial- und Gesundheitswesen entwickelt und diese Roboter z.B. in Pflegeeinrichtungen und anderen Settings genutzt.

Mit dem Aufkommen von Tablets und Smartphones gibt es zunehmend sogenannte Gesundheits-Apps, die eine Vielzahl von Bereichen abdecken. Das Potenzial dieser Apps ist noch lange nicht ausgelotet und sie sind - ebenso wie Assistive Technologien - hinsichtlich ihrer Wirkungen untererforscht.

Ende 2019 trat das Gesetz für eine bessere Versorgung durch Digitalisierung und Innovation (Digitale Versorgungs-Gesetz, DVG) in Kraft, mit dem GesundheitsApps in Zukunft verordnet werden können. Die nächsten Jahre werden zeigen, was dieses für die Gesundheitsversorgung und die Aufrechterhaltung von Autonomie und Teilhabe sowie Lebensqualität bedeuten wird. Die Digitalisierung wird dabei auch die Arbeitsprozesse und -strukturen der Gesundheitsdienstleister wesentlich verändern.

Diese Entwicklungen sind bislang meist ansatzweise in die Aus-, Fort- und Weiterbildung der Gesundheitsberufe aufgegriffen worden. Hier besteht ein großer Bedarf, sollen die Investitionen in die Technologien nicht wirkungslos verpuffen.

\section{Akzeptanz Assistiver Technologien}

Auch wenn das richtige Hilfsmittel verordnet wurde, bedeutet es nicht, dass es von den betroffenen Personen genutzt wird. Eine wesentliche Rolle spielt das sog. sozio-technische System/Arrangement, das die Wechselwirkung von Technik und Umfeld beschreibt (vgl. Weyer 2008, 37ff.). Betrachtet man die verschiedenen Ebenen, um zu erklären, unter welchen Umständen Technik zum Einsatz kommt, so spielen auf der Mikroebene persönliche Faktoren und Handlungswissen zum Umgang mit dem Hilfsmittel eine Rolle. Auf der Mesoebene sind es die dort angesiedelten, komplexen organisatorischen Aspekte, die in der Regel mit dem Erwerb und der Nutzung des Hilfsmittels einhergehen. Auf der Makroebene betrifft es die gesellschaftlichen Steuerungsfragen und die Gestaltung der politischen Rahmenbedingungen (vgl. Klein u.a. 2015, 151). 
Speziell in Bezug auf Hilfsmittel entwickelte Scherer in den 1980er-Jahren das Matching Person and Technology Model (MPT), welches „das Ziel [hat], eine ideale Übereinstimmung zwischen der Person und dem Hilfsmittel zu erreichen“ (Scherer \& Sax 2010, 1322). Dazu müssen drei Basiskomponenten - die Person, die Umwelt und die Technologie - im Prozess der Hilfsmittelversorgung beachtet werden (vgl. ebd., zit. nach Bruckmann u.a. 2015, 20). Hier wird das sozio-technische System ins Blickfeld miteinbezogen. Das dazu entwickelte Assessment-Instrument berücksichtigt die Basiskomponenten „Person“, „Umwelt/Einsatzgebiet“ und „Assistive Technologie/Hilfsmittel“. Der beschriebene Hilfsmittelprozess ist in sieben Schritte gegliedert (vgl. ebd., 20) und ähnelt - dadurch, dass eine Evaluation mit ggf. einer Anpassung erfolgt - dem vierstufigen Regelkreis des PDCAZyklus (plan, do, check, act) im Qualitätsmanagement.

Auf der Basis dieses MPT-Modells wurde das Assistive Technology Device Predisposition Assessment (ATD PA) entwickelt, das dazu dient, den Hilfsmittelbedarf zu analysieren, „das passende Hilfsmittel auszuwählen und einem Nichtgebrauch vorzubeugen" (Cordes 2016, 34). Die Besonderheit des ATD PA ist, dass neben Informationen zur Versorgungssituation auch die psychosozialen Belange in Bezug auf die potenzielle Nutzung des Hilfsmittels erfasst werden. Der ATD PA kann bei Personen mit den unterschiedlichsten Diagnosen und unabhängig vom Alter eingesetzt werden und umfasst neun Formulare für die verschiedenen Bereiche des Hilfsmittelprozesses. Bekannt ist das Verfahren bei den Ergotherapeut*innen, eignet sich jedoch auch für andere Berufsgruppen (vgl. ebd., 36).

Die Deutsche Vereinigung für Rehabilitation (DVfR) konstatiert in ihrem Positionspapier zur Hilfsmittelversorgung aus dem Jahr 2009, dass es für den deutschsprachigen Raum kaum verfügbare Assessment-Instrumente gibt (vgl. DVfR 2009, 42). In dem Positionspapier werden unterschiedliche Ziele in der Hilfsmittelversorgung aufgezeigt: Hilfsmittel können zum einen die ärztliche Behandlung unterstützen oder zur Beseitigung von Behinderungen eingesetzt werden. Auf der anderen Seite können Hilfsmittel zum Ausgleich einer Behinderung dienen oder zur Vermeidung, Verminderung oder Kompensation von Behinderungen beitragen (ebd., 14). Neben der Teilhabeorientierung wird bei der Hilfsmittelversorgung auch eine Sozialraumorientierung gefordert, bei der sich an Kriterien wie „Kompetenz/Qualifikation, Erreichbarkeit, Verfügbarkeit und am Gesamtverlauf der Hilfsmittelversorgung" (ebd., 22) orientiert werden soll.

„Begreift man die Hilfsmittelversorgung als Möglichkeit der Problemlösung für Menschen mit Behinderung, dürften überwiegend regionale, wohnortnahe bzw. sozialräumlich gestaltete Strukturen sinnvoll sein, die feste Kooperationsbeziehungen und Vernetzung ermöglichen und eine Qualitätskontrolle erleichtern.“ (ebd.)

Deutlich wird, dass die verschiedenen Ansätze in unterschiedlicher Weise dazu beitragen, die Hilfsmittelversorgung und -akzeptanz zu verstehen. Mit dem MPT- 
Modell und dem dazugehörigen ATD-PA-Assessment steht ein Verfahren für die berufliche Praxis zur Verfügung, das den Anforderungen der DVfR und der ICF zu entsprechen scheint.

Wünschenswert ist eine wissenschaftliche Evaluation des Einsatzes und Gebrauchs von (schon vorhandenen und zu entwickelnden) Hilfsmitteln in ihrem sozio-technischem System - also auch mit den potenziellen Nutzer*innen -, um somit tatsächlich zu einer adäquaten Produktentwicklung zu kommen und zu einer Verbesserung der Autonomie und Lebensqualität von betroffenen Menschen beitragen zu können.

\section{Information und Qualifizierung}

Assistive Technologien bzw. Hilfsmittel sind kein verbindliches Curriculum in der medizinischen Ausbildung, obwohl Ärzt*innen die einzigen sind, die Hilfsmittel verordnen dürfen. Gesundheitsberufe, mit denen (mehr oder weniger eng) kooperiert wird, sind die pflegerischen, sozialarbeiterischen, ergotherapeutischen, logopädischen und orthopädischen Fachberufe, Hörgeräteakustiker*innen, Optiker*innen, Orthoptist*innen etc. All diese Berufe müssen sich nicht nur auf die technologischen Veränderungen einstellen, sondern auch auf die damit verbundenen prozessbezogenen Veränderungen in der Dienstleistungserbringung. Hier stellt sich die Frage, welche Qualifizierungsmaßnahmen erforderlich sind und wie die betroffenen Menschen und ihre Angehörigen sowie die Gesundheitsprofession entsprechend informiert und qualifiziert werden können.

Neben rehabilitationsbezogenen Ausbildungs- und Studiengängen in Deutschland gibt es an der Frankfurt University of Applied Sciences (Frankfurt UAS) den interdisziplinären Masterstudiengang Inclusive Design - Zukunft interdisziplinär gestalten, der alles Wissenswerte zu Assistiven Technologien und barrierefreien Systemen vermittelt, damit Menschen unabhängig von Alter und möglichen funktionalen Einschränkungen selbstbestimmt und selbstständig leben können. Der interdisziplinäre Masterstudiengang kombiniert die drei Profile Inklusive Architektur, Intelligente Systeme sowie Digital Health und Case Management durch gemeinsam durchgeführte Projekte und erweitert das fachspezifische Wissen um interdisziplinäre Methoden und Inhalte wie z.B. partizipative Forschungsdesigns oder Fragestellungen der Akzeptanz und Ethik.

Der interdisziplinäre Masterstudiengang Inclusive Design ist eingebunden in die Forschungsaktivitäten des interdisziplinären Forschungszentrums FUTURE AGING und dem Innovation Lab 5.0 sowie der (Dauer-)Ausstellung „Hallo Freiheit! Zusammen über Barrieren“, die gemeinsam mit der Frankfurter Stiftung für Gehörlose und Schwerhörige und dem Sozialverband Hessen-Thüringen betrieben wird. 
Diese Ausstellung informiert interaktiv und mittels dialogorientierter Führungen zu den Themen Assistive Technologien und Barrierefreiheit sowie rund um den Themenkomplex Gehörlosigkeit und Schwerhörigkeit.

Besucher*innen können die jeweiligen Hilfsmittel sehen, fühlen und ausprobieren, um somit ein besseres Verständnis der Funktionalitäten und möglicher Unterstützungspotenziale zu erhalten. Dialogorientierte Führungen ermöglichen sowohl den betroffenen Menschen und ihren Angehörigen als auch den Fachkräften einen intensiven Austausch. Mit einem sog. Alterssimulationsanzug können verschiedene Funktionsverluste simuliert und selbst erfahren werden, sodass damit eine erste Sensibilisierung zu den verschiedenen Funktionsverlusten ermöglicht wird. Die Erkenntnisse führen zu einem besseren Verständnis altersbezogener Prozesse sowie einem daraus resultierenden veränderten Verhalten.

Während die Ausstellung „Hallo Freiheit! Zusammen über Barrieren “sich vor allem auf die Möglichkeiten in der häuslichen Umgebung konzentriert, hat das an der Frankfurt UAS ansässige Innovation Lab 5.0 den Arbeitsplatz der Zukunft der Gesundheitsakteure im Blick.

Um mit den technologischen Entwicklungen Schritt zu halten, werden Anwendungsszenarien für eine gute Versorgung bei Funktionsverlusten entwickelt und exemplarisch in der Ausstellung (und auch in Verbindung mit dem Innovation Lab 5.0) dargestellt. Die Ergebnisse fließen dabei in die Weiterentwicklung der Themen ein. Der dazugehörige YouTube-Kanal ${ }^{2}$ präsentiert ergänzend in kurzen Videoclips Hintergrundinformationen zu den verschiedenen Produkten und Konzepten.

Die Idee des „touch, feel and learn“, das im Rahmen der Ausstellung „Hallo Freiheit! Zusammen über Barrieren " und dem Innovation Lab 5.0 umgesetzt wurde, erweist und erwies sich als eine sehr gute Voraussetzung zum Verständnis der Möglichkeiten von Assistiven Technologien für ein selbstständiges Leben und Teilhabe. Da es keine flächendeckende Verbreitung solcher Ausstellungen gibt, wurde das Prinzip im Projekt CareV.E.T. (Innovative Training approach for Supportive Living Operators; Erasmus+ Strategic Partnership for vocational education and training, 2015-2018) aufgegriffen und eine zertifizierte Weiterbildungsmaßnahme mit einem sensorbasierten Toolkit entwickelt und erprobt. Zielgruppen dieser Weiterbildungsmaßnahme sind Fachkräfte, die in Einrichtungen für Menschen mit Behinderungen arbeiten. Das Toolkit, das aus einer internetbasierten Plattform und der Anbindung unterschiedlicher sensorbasierter Produkte besteht, wird im Rahmen des Projektes DDSKILLs (Cutting-Edge Digital Skills for professional caregivers of Persons with Disabilities and Mental Health Problems) auf die Anbindung von Telepräsenzrobotik und Virtual Reality wie auch Augmented Reality erweitert. Dazu werden die Weiterbildungsinhalte weiterentwickelt, so dass 2022 eine zertifizierte Weiterbildungsmaßnahme als MOOC (Massive Open

2 www.youtube.com/barrierefreieswohnen 
Online Course) zu diesen technologischen Neuerungen in mehreren europäischen Ländern zur Verfügung stehen wird und für die oben genannte Zielgruppe ausgerichtet ist.

\section{Schlussfolgerung und Diskussion}

Der Bereich Assistive Technologien ist durch eine Vielfalt an Hilfsmitteln, schnellen technologischen Entwicklungen und einer komplexen Regelungsstruktur gekennzeichnet. Hilfsmittel werden von Ärzt*innen verordnet, die diese Thematik bislang noch nicht fest in ihrem Ausbildungskanon haben. Eine Zusammenarbeit mit anderen Gesundheitsfachberufen ist dabei zwar sinnvoll (und gibt es häufig), wird aber nicht regelhaft umgesetzt. Dieses kann zu „Fehl“-Verordnungen führen, mit der Folge einer nicht zufriedenstellenden Versorgung für die betroffenen Menschen. Die Frankfurt UAS geht diese Fragestellungen auf mehreren Ebenen an: Mit dem interdisziplinären Forschungszentrum FUTURE AGING wird zu Assistiven Technologien und inklusiver Architektur geforscht. Der interdisziplinäre Masterstudiengang Inclusive Design qualifiziert die Gesundheitsberufe, Architekt*innen und Informatiker*innen zu Assistiven Technologien und barrierefreien Umgebungen. Mit der Ausstellung „Hallo Freiheit! Zusammen über Barrieren“ und dem Innovation Lab 5.0 können die Produkte unmittelbar kennengelernt und erprobt werden. Des Weiteren bieten die Räumlichkeiten und die adaptierbare Ausstattung auch die Möglichkeit, die sich durch die Technologien ergebenden Veränderungen in den Arbeitsprozessen der involvierten Dienstleistungsberufe durchzuspielen und zu erproben. Darüber hinaus wird eine zertifizierte Weiterbildungsmaßnahme erarbeitet, die mittels eines Toolkits die technologischen Veränderungen erfahrund verstehbar macht. Diese wird in mehreren europäischen Ländern (auch in Deutschland) erprobt und zertifiziert, so dass dieser Themenbereich in der beruflichen Weiterbildung angeeignet werden kann.

\section{Literatur}

AARP (2017): The Aging Readiness Competitiveness Report. Germany. Online unter: http://www.silvereco.org/en/wp-content/uploads/2017/12/ARC-Report-Germany.pdf. (Abrufdatum: 19.01.2020).

Bruckmann, N. u.a. (2015): MPT \& ATD PA Matching Person and Technology Model (MPT-Modell) und Assistive Technology Device Predisposition Assessment (ATD PA). Ein klientenzentrierter Wegweiser für die Hilfsmittelberatung und -versorgung in Deutschland. Idstein: Schulz-Kirchner Verlag GmbH.

Connell, J. u.a. (2008): Comprehensive scoping study on the use of assistive technology by frail older people living in the community. Sydney: Urbis for the Department of Health and Aging.

Cordes, A. (2016): Alltagsbegleiter maßschneidern. Assessment für die Hilfsmittelversorgung. In: ergopraxis $9(6), 34-36$. 
DIN Institut für Normung e.V. (2017): DIN EN ISO 9999. Hilfsmittel für Menschen mit Behinderungen - Klassifikation und Terminologie (ISO 9999: 2016); Deutsche Fassung EN ISO 9999:2016. DIN-Normenausschuss Medizin (NAMed). Berlin: Beuth Verlag GmbH.

DVfR - Deutsche Vereinigung für Rehabilitation (2009): Überwindung von Problemen bei der Versorgung mit Hilfsmitteln - Lösungsoptionen der DVfR. Online unter: https://www.dvfr.de/ fileadmin/user_upload/DVfR/Downloads/Stellungnahmen/DVfR_L\%C3\%B6sungsoptionen_ Hilfsmittelversorgung_Okt._2009.pdf. (Abrufdatum: 13.01.2019) (auch erschienen in: Die Rehabilitation 2010, 49, 5-36).

GKV-Spitzenverband (2019): 2. Bericht des GKV-Spitzenverbandes zur Fortschreibung des Hilfsmittelverzeichnisses gemäß $\$ 139$ Absatz 9 Satz 3 SGB V. Online unter: https://www.gkv-spitzenverband.de/media/dokumente/krankenversicherung_1/hilfsmittel/fortschreibungen_aktuell/20190226_2._Hilfsmittelfortschreibungsbericht_GKV-SV.pdf. (Abrufdatum: 12.04.2020).

Hilfsmittel-Richtlinie/HilfsM-RL (2018): Richtlinie des Gemeinsamen Bundesausschusses über die Verordnung von Hilfsmitteln in der vertragsärztlichen Versorgung. Veröffentlicht im Bundesanzeiger BAnz AT 02.10.2018 B2. Online unter: https://www.gkv-spitzenverband.de/media/dokumente/krankenversicherung_1/hilfsmittel/Hilfsmittel-Richtlinie_Stand_19-07-2018.pdf. (Abrufdatum: 29.12.2019).

Institut der Deutschen Wirtschaft Köln e.V. (o.J.): Über REHADAT-Hilfsmittel. Online unter: https://www.rehadat-hilfsmittel.de/de/informationen/ueber-uns. (Abrufdatum: 31.01.2020).

Klein, B., Reutzel S. \& Roßberg, H. (2015): Zur Mediatisierung assistiver Technologien - der Hausnotruf als Kommunikationsmedium für ältere Menschen. In: N. Kutscher, T. Ley \& U. Seelmeyer (Hrsg.): Mediatisierung (in) der Sozialen Arbeit. Band 38. Baltmannsweiler: Schneider Verlag Hohengehren, 151-168.

Klein, B. u.a. (2018): Robotik in der Gesundheitswirtschaft. Einsatzfelder und Potenziale. Heidelberg: medhochzwei Verlag.

Postbank (2019): Umfrage: Surfen ist mehr als ein Vollzeitjob. Postbank Digitalstudie 2019. Online unter: https://www.postbank.de/postbank/pr_presseinformation_2019_05_20_umfrage_surfen_ ist_mehr_als_ein_vollzeitjob.html. (Abrufdatum: 21.02.2020).

Scherer, M.J. \& Sax, C. (2010): Measure of Assistive Technology Predisposition and Use. In: E. Mpofu \& T. Oakland (Eds.): Rehabilitation and Health Assessment: Applying ICF-Guidelines. New York: Springer Publishing Company, LLC, 229-254.

Statistisches Bundesamt (2019): Bevölkerung im Wandel. Annahmen und Ergebnisse der 14. koordinierte Bevölkerungsvorausberechnung. Wiesbaden: Eigenverlag.

Statistisches Bundesamt (2018a): Pflegestatistik 2017. Pflege im Rahmen der Pflegeversicherung. Deutschlandergebnisse. Wiesbaden: Eigenverlag.

Statistisches Bundesamt (2018b): Statistik der schwerbehinderten Menschen. Kurzbericht 2017. Wiesbaden: Eigenverlag.

Statistisches Bundesamt (2015): Bevölkerung Deutschlands bis 2060. 13. koordinierte Bevölkerungsvorausberechnung. Wiesbaden: Eigenverlag.

Stainhauser, E. (2019): Nutzererlebnis älterer Menschen mit einem Hausnotrufdienst über den Sprachassistenten Amazon Alexa. Unveröffentl. Masterarbeit im Masterstudiengang Barrierefreie Systeme, Frankfurt University of Applied Sciences.

Weyer, J. (2008): Techniksoziologie. Genese, Gestaltung und Steuerung sozio-technischer Systeme. Weinheim: Juventa.

WHO Centre for Health Development (Kobe, Japan). (2004): A Glossary of Terms for Community Health Care and Services for Older Persons. Ageing and Health Technical Report Vol. 5. Online unter: https://apps.who.int/iris/handle/10665/68896. (Abrufdatum: 12.04.2020). 


\section{Planungs- und Gestaltungsprozesse}

\section{Christophe Kunze \\ Nutzerorientierte und partizipative Ansätze in \\ Gestaltungs- und Aneignungsprozessen von teilhabefördernder Technik}

\section{Kontext: Digitaler Wandel und Teilhabe}

Die Digitalisierung führt zu tiefgreifenden Veränderungen in den wirtschaftlichen, sozialen, kulturellen und politischen Strukturen unserer Gesellschaft. Digitalisierung verändert unser Verhalten, etwa wie wir einkaufen, Kontakte knüpfen oder arbeiten. Sogenannte „disruptive Entwicklungen“ führen in kurzer Zeit zu tiefgreifenden Veränderungen im Bereich der Wirtschaft, von denen in zunehmendem Maße auch das Sozial- und Gesundheitswesen erfasst werden. Für die Teilhabe von Menschen mit Behinderung ist der digitale Wandel auf zwei Ebenen von Bedeutung:

- Teilhabe an der digitalen Welt: Zum einen wird die Teilhabe am gesellschaftlichen Leben immer mehr auch über digital vermittelte Interaktionen realisiert, welche die Nutzung von digitalen (Online-)Medien voraussetzen. Digitale Technologien bieten dabei potenziell vielfältige Chancen zur Förderung der Teilhabe von Menschen mit Behinderung. So können Menschen mit Mobilitätseinschränkungen durch ortsunabhängige Nutzung von Onlinediensten neue Teilhabemöglichkeiten erschließen, und soziale Medien bieten häufig stigmatisierungsarme Interaktionsräume. Gleichzeitig tragen Menschen mit Behinderung aber ein hohes Risiko, durch Ungleichheiten in der Nutzung digitaler Technologien („digital divide“) benachteiligt zu werden. Insbesondere Menschen mit Lernschwierigkeiten sowie Menschen, die in Einrichtungen der Behindertenhilfe leben, weisen eine deutlich geringere Nutzung von Internetdiensten auf als der Bevölkerungsdurchschnitt (vgl. Haage \& Bosse 2019).

- Teilhabe durch Digitalisierung: Zum anderen können digitale Technologien als Hilfsmittel die Teilhabe in der physischen (Um-)Welt unterstützen, indem 
sie entweder Funktionseinschränkungen kompensieren oder helfen, Barrieren zu überwinden. Neben speziell für Menschen mit Behinderung entwickelten Hilfsmitteln, sog. assistiven Technologien (vgl. Klein in diesem Band), nimmt dabei auch die teilhabefördernde Nutzung von Consumertechnik eine immer größere Rolle ein (vgl. Kunze 2019). Ein typisches Beispiel hierfür sind teilhabefördernde Applikationen auf Smartphones, wie z.B. Vorlesesysteme, Bilderkennungssysteme oder Navigationsdienste (vgl. Doughty 2011).

Auch wenn die Potenziale digitaler Technologien zur Förderung der Teilhabe unbestritten sind, erfüllen technische Assistenzsysteme häufig nicht die in sie gesetzten Erwartungen. Viele der umfassenden Forschungsaktivitäten der letzten Jahre mündeten nicht in praktisch nutzbare Systeme und Anwendungen. Aber auch die technischen Entwicklungen, die in markttaugliche Produkte überführt wurden, führen in der Praxis nicht immer zu mehr Teilhabe. Schätzungen gehen davon aus, dass auch bei schon länger etablierten Technologien ca. $30 \%$ der ausgelieferten Hilfsmittel nicht genutzt werden (vgl. Federici, Meloni \& Borsci 2016). Häufig gehen vor allem technikgetriebene Entwicklungsprojekte auch am Bedarf der Zielgruppe vorbei, etwa durch eine defizitorientierte Fokussierung auf Funktionseinschränkungen oder auf Sicherheitsbedürfnisse von Betreuungspersonen an Stelle von Teilhabezielen der Betroffenen (vgl. Fitzpatrick u.a. 2015). Eine wesentliche Innovationsbarriere ist die häufig unterschätze Komplexität des Technikeinsatzes in der Versorgung (siehe Abschnitt 3.2).

Im Diskurs zu assistiven Technologien wird die Ursache für derartige Fehlorientierungen meist in einer unzureichenden Einbindung der Zielgruppen in Forschungsund Entwicklungsprojekte gesehen. In der technischen Forschung hat dies in den letzten Jahren zu einem Paradigmenwechsel hin zu nutzerorientierten und partizipativen Gestaltungsprozessen geführt (vgl. Kunze 2017). Im vorliegenden Beitrag werden dazu grundlegende methodische Ansätze sowie spezifische Herausforderungen im Kontext von Menschen mit Behinderung als Zielgruppe der Technikentwicklung thematisiert. Neben den Gestaltungsprozessen assistiver Technologien werden dabei auch Aneignungsprozesse von Consumertechnik für soziotechnische Arrangements zur Förderung der Teilhabe in den Blick genommen.

\section{Nutzerorientierte Technikgestaltung aus dem Blickwinkel inklusiver Forschung}

\subsection{Nutzerorientierte Technikgestaltung: Methodische Ansätze und Umsetzung in der Forschungspraxis}

Während in der klassischen Technikentwicklung das Hauptaugenmerk auf technische Funktionalitäten und deren Realisierbarkeit gerichtet wird, stehen in der nutzerorientierten Technikgestaltung die Anwender*innen von Technologien und 
deren Bedarfe und Ziele im Mittelpunkt. Da neben den eigentlichen Nutzenden eines technischen Systems oft auch andere Personen von technischen Veränderungen betroffen sind, spricht man heute oft auch von mensch-zentrierter Gestaltung. Hierzu haben sich verschiedene methodische Ansätze etabliert, die sich entweder im engeren Sinne auf die Gestaltung der Mensch-Technik-Interaktion beziehen (so z.B. in der Gebrauchstauglichkeitsnorm ISO 9410-210), oder aber in breiterer Perspektive auf die Konzeption von völlig neuen Produkt- und Dienstleistungskonzepten abzielen (z.B. „Design Thinking“-Ansatz). Eine Gemeinsamkeit dieser Ansätze ist, dass die Entwicklung typischerweise als iterativer Prozess verstanden wird, in dem sich Phasen der Nutzungskontextanalyse (z.B. durch teilnehmende Beobachtung, Interviews, Analyse), Kreativitätsphasen zur Exploration des Lösungsraums (z.B. über Design Workshops oder Prototyping) und Testphasen zur frühzeitigen Gewinnung von Nutzungserfahrungen (z.B. Labor- oder Feldtests) abwechseln.

Das Ziel der Nutzungskontextanalyse ist im Allgemeinen, Beschreibungen typischer Nutzer*innen mit ihren Eigenschaften, Aktivitäten, Bedarfen und Problemen zu erstellen, die als Grundlage des Gestaltungsprozesses dienen. Die Betrachtung erfolgt dabei auf der Ebene von Nutzer*innengruppen. Bei der Betrachtung von Technik zur Förderung der Teilhabe von Menschen mit Behinderung wird man dabei in der Regel auf eine große Diversität von Anwendungskontexten und sehr heterogene Zielgruppen treffen. Im Kontext der Barrierefreiheit hat sich dazu das Gestaltungsprinzip des Universal Design etabliert. Nach Art. 2 der UN-BRK meint Universal Design „ein Design von Produkten, Umfeldern, Programmen und Dienstleistungen in der Weise, dass sie von allen Menschen möglichst weitgehend ohne eine Anpassung oder ein spezielles Design genutzt werden können“; das schließt „Hilfsmittel für bestimmte Gruppen von Menschen mit Behinderung, soweit sie benötigt werden, nicht aus“. Diese Ergänzung ist wichtig, da sich Universal Design („Design for All") ebenso wie Barrierefreiheit auf der Umsetzungsebene i.d.R. auf Anforderungen von Gruppen bezieht (vgl. Welti in diesem Band). Für den Einzelfall beruhen soziotechnische Arrangements zur Förderung der Teilhabe daher in der Regel auf einer Kombination aus Barrierefreiheit, Universal Design und Ergänzungen durch individuelle Anpassungen, die gemeinsam einen Lösungsraum als „Continuum of Solutions" bilden (vgl. Bühler 2009, zit. nach Haage \& Bühler 2019). Für digitale Technologien erscheint dieser Aspekt besonders relevant, da diese anders als z.B. die gebaute Umwelt relativ einfach und zum Teil auch dynamisch (d.h. zur Nutzungszeit) an individuelle Anforderungen und spezifische Nutzungskontexte angepasst werden können.

Die Methoden des nutzerzentrierten Designs können jedoch dazu verleiten, in der Nutzungskontextanalyse Beobachtungen zu stereotypen Beschreibungen von „typischen Nutzer*innen“ zu verdichten, die der Diversität der Nutzenden nicht mehr gerecht werden. Die Vielfalt und Widersprüchlichkeit von Alltagspraktiken von Menschen können nur bedingt in Kategorien abgebildet werden. Im Kon- 
text des Inclusive Designs werden deshalb als Kontrapunkt zum „Design for All“Ansatz zusätzlich auch individuelle Gestaltungsprozesse bis hin zu Einzelfällen („Design for $\mathrm{Me}^{\prime)}$ vorgeschlagen (vgl. Magnusson, Hedvall \& Breidegard 2018; Matiouk 2019). „Design for All“ und „Design for me“ sind dabei nicht als gegensätzliche, sondern als komplementäre Ansätze zu verstehen.

Eine weitere typische Schwierigkeit im Nutzerzentrierten Design ist die Tatsache, dass sich der Gestaltungsprozess in der Regel auf Erhebungen zu bestehenden Alltagspraktiken und -problemen der adressierten Zielgruppe stützt. Gerade im Kontext von digitalen Technologien bleibt dabei aber weitgehend im Unklaren, wie sich Nutzungskontexte und -bedingungen durch neue technische Lösungen verändern, was insbesondere bei disruptiven Entwicklungen zu völlig anderen Situationen führen kann (vgl. Peine u.a. 2014). Es ist daher empfehlenswert, möglichst früh im iterativen Gestaltungsprozess auch Nutzungserfahrungen mit prototypischen technischen Lösungen zu erheben (vgl. Wulff u.a. 2015). Zwei mögliche Ansätze, die sich hierzu bewährt haben, sind „Technology Probes“ und „Wizardof-Oz-Experimente“. Der Technology Probes-Ansatz (vgl. Hutchinson u.a. 2003) beruht auf Fallstudienbeobachtungen mit einfachen, flexibel nutzbaren und adaptierbaren Standardtechnologien, die Probanden der Zielgruppe zur Verfügung gestellt werden, um Aneignungsprozesse und daraus resultierende veränderte Anwendungskontexte zu untersuchen. Wizard-of-Oz-Experimente (vgl. Dahlbäck, Jönsson \& Ahrenberg 1993) sind Studien zu Interaktionen von Probanden mit scheinbar autonomen intelligenten Systemen, deren Verhalten aber im Verborgenen von einem Menschen gesteuert wird. Diese ermöglichen eine frühzeitige Untersuchung von Anwendungsideen, deren prototypische Realisierung deutlich mehr Aufwand erzeugen würde. Abbildung 1 zeigt ein Wizard-of-Oz Experiment mit Menschen mit Demenz beim Sitztanz. Die Anwendung erweitert den Sitztanz (scheinbar) derart, dass mit Hilfe von Trittmatten die Bewegung beim Sitztanz die Musik steuert. Die Musikwiedergabe wird im Experiment in Wirklichkeit per Smartphone gesteuert (vgl. Bejan u.a. 2017).

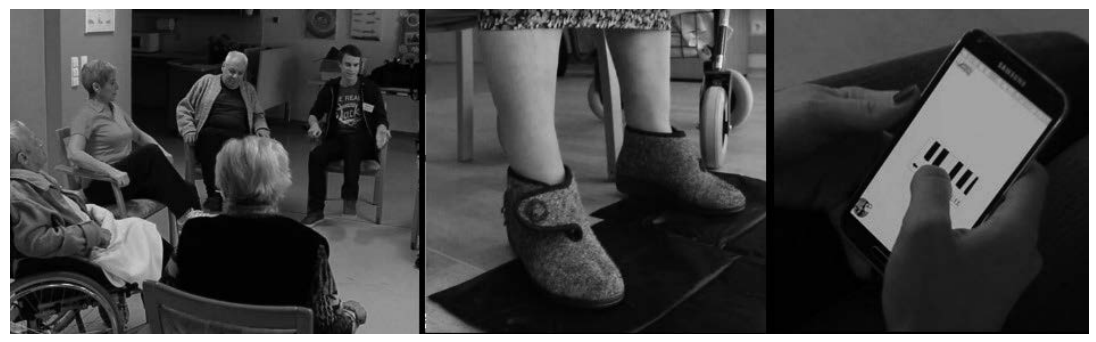

Abb. 1: Beispiel für einen Wizard-of-Oz-Test mit Menschen mit Demenz zur Anwendung „Augmentierter Sitztanz" 
Insgesamt zeigen die Erfahrungen aus Forschungsprojekten zur Gestaltung teilhabefördernder Technik, dass aus konzeptionellen Überlegungen heraus entwickelte Technikanwendungen in der Praxis häufig nicht funktionieren, wobei die Gründe hierfür sehr vielfältig sein können. Aus diesem Grund ist eine möglichst praxisbasierte Forschung zu teilhabefördernder Technik einzufordern, wobei eine praktische Nutzung neuer Technologien unter realistischen Anwendungsbedingungen Mittelpunkt und Ausgangsbasis des Forschungsprozesses sein sollte.

\subsection{Technikgestaltung und partizipative Forschung: Chancen und Herausforderungen}

Nutzerorientierte Technikgestaltung ist, ebenso wie inklusive Forschung, eng verknüpft mit partizipativen Ansätzen. Die heute im Bereich der Technikgestaltung typischen Ansätze des partizipativen Designs (häufig auch als „Co-Design“ bezeichnet) haben ihre Wurzeln in der skandinavischen Design-Schule und Projekten im Kontext der Gestaltung von Arbeitssystemen (vgl. Kensing \& Blomberg 1998). Neben dem Recht auf Mitgestaltung von Arbeitskontexten liegt eine Motivation für Co-Design-Prozesse in der Annahme, dass Nutzer*innen als Expert*innen in eigener Sache zu besseren Ergebnissen des Designprozesses beitragen können. In der partizipativen Technikgestaltung werden Nutzende aktiv in die Ko-Produktion von Wissen und Gestaltungsartefakten (z.B. über partizipative Design-Workshops) eingebunden. Technikgestaltung ist dabei als iterativer Aushandlungsprozess zwischen Technikentwicklern, Nutzenden und weiteren Stakeholdern zu verstehen.

Insbesondere in der öffentlich geförderten Forschung zur Technikgestaltung im Kontext gesellschaftlicher Herausforderungen (z.B. Demografischer Wandel oder Pflege) werden partizipative Ansätze heute i.d.R. eingefordert und sind vielfach etabliert. Die dabei realisierten Aktivitäten entsprechen allerdings oft nicht dem Verständnis von Partizipation und den Ansprüchen an Partizipation im Sinne inklusiver Forschung (vgl. Goeke 2016). Zum einen werden meist niedrige Stufen der Beteiligung wie Beratung oder Mitwirkung in ausgewählten Phasen der Projektdurchführung realisiert; eine gleichberechtigte Zusammenarbeit oder gar Steuerung des Forschungsprozess finden in der Regel nicht statt (vgl. dazu die Orientierungsmatrix der DVfR/DGRW: Farin-Glattacker u.a. 2014). Vor allem aber werden einer ausreichenden Auswahl, Begründung und Reflexion der Partizipationsprozesse (z.B. in Bezug auf Haltung, Zielkonflikte und Machtverhältnisse), der Barrierefreiheit des Forschungsprozesses sowie der Befähigung (Capacity Building) der eingebundenen Betroffenen typischerweise kaum Aufmerksamkeit geschenkt. In Bezug auf partizipative Technikgestaltung ließe sich vereinfacht sagen: Nicht überall, wo Partizipation draufsteht, ist auch Partizipation drin. Partizipative Technikgestaltung für und mit Menschen mit Behinderung unterliegt wie inklusive Forschung im Allgemeinen besonderen Herausforderungen. 
Die Aushandlungsprozesse als Grundlage des Co-Designs sind auf explizite Repräsentationen von Nutzungskontexten und Gestaltungskonzepten angewiesen. Hierfür werden typischerweise z.B. textuelle Szenarien, Storyboards, grafische notierte formale Modelle (z.B. Prozessmodelle), Videoszenarien, oder visuelle Darstellungen von Realisierungsalternativen (z.B. sog. Software-Mock-Ups) verwendet. Diese Aushandlungsartefakte sind im Allgemeinen nicht barrierefrei, so dass im Kontext des Inclusive Design häufig alternative und ggf. experimentelle Darstellungsformen und Partizipationsmethoden entwickelt werden müssen. Häufig wird auch auf eine Partizipation über Stellvertreter*innen zurückgegriffen, was auf Grund der Gefahr von Verzerrungen oder Interessenskonflikten eine angemessene Reflexion der Ergebnisse des Partizipationsprozesses erfordert. In der wissenschaftlichen Literatur sind hilfreiche Projekterfahrungen zum Umgang mit den Herausforderungen und Widersprüchlichkeiten der partizipativen Technikgestaltung für und mit Menschen mit Behinderung vereinzelt dokumentiert (vgl. Borges u.a. 2016; Haworth u.a. 2016; Rajapakse 2018). Wünschenswert wären für die Zukunft hierzu Sammlungen von Beispielen guter Praxis sowie Leitfäden und Empfehlungen für die Forschungspraxis.

\section{Aneignungsprozesse teilhabefördernder Technik}

\subsection{Teilhabe und Aneignung von Consumertechnik}

Wie schon in Abschnitt 1 dargestellt, ist eine teilhabefördernde Nutzung von Technik nicht auf Assistive Technologien, also speziell für diesen Zweck entwickelte Systeme, beschränkt. Im Gegenteil: Im Kontext des digitalen Wandels nimmt die Bedeutung von allgemeiner Consumertechnik („mainstream technology") für die Teilhabe von Menschen mit Behinderung erheblich zu. Beispiele hierfür sind Smartphones, „smarte“ vernetzte Objekte („Internet der Dinge“) und Anwendungen künstlicher Intelligenz. Das ist zum einen darin begründet, dass die Zugänglichkeit vieler Massenprodukte durch fortschrittliche Bedienhilfen auBerordentlich hoch ist (z.B. Smartphones). Zum anderen können digitale Produkte und Anwendungen häufig umfassend angepasst und verknüpft werden. Häufig werden dabei auch bestehende Hard- und Softwaresysteme auf kreative Weise zweckentfremdet, um eine teilhabefördernde Wirkung zu erzielen.

Da diese Produkte nicht speziell für Menschen mit Behinderung und nicht für spezifische Nutzungskontexte entwickelt werden, können die in Abschnitt 2 beschriebenen Ansätze zur nutzerorientierten und partizipativen Technikgestaltung nicht direkt für den teilhabefördernden Einsatz von Consumertechnik angewendet werden. Sie lassen sich aber in ähnlicher Weise auf den Prozess der Technikaneignung übertragen. Denn die teilhabefördernde Wirkung von Consumertechnik entsteht in der Regel nicht allein aus konzeptionellen Überlegungen oder 
aus guter Gestaltung der Technik, sondern beruht im Allgemeinen auf kontinuierlichen Aneignungsprozessen mit der Anpassung von Technikarrangements an Alltagspraktiken und umgekehrt. Tatsächlich sind Menschen mit Behinderung und ihre Hilfspersonen in ihrer täglichen Praxis auch heute schon daran gewöhnt, Arbeitsprozesse, Routinen und technische Artefakte pragmatisch wechselseitig aneinander anzupassen (vgl. Mol, Moser \& Pols 2010). Digitale Technik kann in diesem Kontext als technische Konfiguration („configurational technology“) betrachtet werden. Dieses Konzept betont die Möglichkeit und Notwendigkeit für die Nutzer*innen, „offene“ vernetzte technische Systeme durch Konfigurationsprozesse an zum Entwicklungszeitpunkt unvorhergesehene individuelle Gegebenheiten anzupassen und dadurch selbst erst konkrete Nutzungskontexte zu spezifizieren (vgl. Peine u.a. 2014; Gomez 2015). Ein Beispiel hierfür stellt die Verknüpfung und Anpassung von vernetzten Objekten über Automatisierungsdienste für das Internet der Dinge dar (vgl. Kunze 2017), wie z.B. die Anbindung eines Sprachassistenten an mobile robotische Systeme zum Drücken von mechanischen Schaltern und Knöpfen. Solchen flexiblen digitalen Technologien kann ein hohes Potenzial zur Förderung der Teilhabe von Menschen mit Behinderung zugesprochen werden, dessen Erschließung allerdings eine Befähigung und entsprechende Rahmenbedingungen zur kreativen Auseinandersetzung mit Technik voraussetzen. Wünschenswert sind daher Aktivitäten, welche die Digitalkompetenzen von Menschen mit Behinderung und deren Bezugs- und Betreuungspersonen fördern, sowie niederschwellige Möglichkeiten zur Gewinnung von Nutzungserfahrungen schaffen (vgl. Mayerle 2015 als Beispiel guter Praxis).

\subsection{Digitale Technologien in Versorgungsstrukturen}

In der nutzerzentrierten Technikgestaltung wird in der Regel das Zusammenspiel von Nutzenden und Technik in individuellen Nutzungskontexten untersucht. Einer erfolgreichen Integration von digitalen Technologien in Versorgungsprozesse und -strukturen im Sozial- und Gesundheitswesen stehen aber im Allgemeinen weitere Barrieren auf Meso- oder Makroebene entgegen, die häufig nicht in ausreichendem Maße berücksichtigt werden. Ein umfassendes Modell zur Erklärung von Implementierungs- und Translationsbarrieren für technische Innovationen im Gesundheitswesen, welches sich auch auf assistive Technologien in der Versorgungspraxis anwenden lässt, stellt das NASSS-Framework (Greenhalgh u.a. 2017; deutsche Übersetzung vgl. Kunze 2020) dar. Nach dem Modell lassen sich die (Nicht-)Nutzung sowie Barrieren für den Transfer, die Verbreitung und die Nachhaltigkeit von technischen Innovationen im Gesundheitswesen i.d.R. durch eine zu hohe bzw. unterschätzte Komplexität soziotechnischer Arrangements auf den verschiedenen Ebenen erklären. Mögliche Barrieren in diesem Kontext können z.B. sozio-kulturelle Akzeptanzfaktoren, neue Qualifikationsanforderungen an Nutzer*innengruppen, Rollen- und Verantwortungsverschiebungen im Versor- 
gungssystem, eine eingeschränkte Veränderungsfähigkeit von Organisationen, die nachhaltige Finanzierung der Techniknutzung oder auch eine schlechte Passung in regulatorische Rahmenbedingungen sein.

Ein Beispiel für entsprechende Barrieren im Kontext von neuen assistiven Technologien stellen Information- und Beratungsbedarfe dar (vgl. Kunze 2019): Viele spezielle technische Hilfsmittel bzw. teilhabefördernde Nutzungsmöglichkeiten von Consumertechnik sind weder Betroffenen noch professionellen Betreuungskräften bekannt, können über lokale Vertriebskanäle wie z.B. Sanitätshäuser nicht beschafft werden und erfordern eine intensive Begleitung bei der Auswahl, Konfiguration und Einführung der Techniknutzung, deren Finanzierung im Fürsorgesystem in der Regel nicht vorgesehen ist. Um eine nachhaltige Nutzung von teilhabefördernder Technik in der Praxis zu fördern, sind daher der Entwicklung von neuen assistiven Technologien ergänzende Forschungs- und Entwicklungsaktivitäten zu sozialen Innovationen notwendig, welche die dafür nötigen Veränderungsprozesse auf institutioneller und gesellschaftlicher Ebene in den Blick nehmen.

\section{Schlussfolgerungen}

Die digitale Transformation hat auf Teilhabemöglichkeiten von Menschen mit Behinderung erhebliche Auswirkungen. Auf der einen Seite ist es notwendig, Zugangs- und Nutzungsbarrieren zur Teilhabe an der digitalen Welt für alle Menschen abzubauen, um zusätzliche Exklusion zu vermeiden. Andererseits sollten Anstrengungen unternommen werden, die Möglichkeiten digitaler Technologien zur Förderung der Teilhabe unter Berücksichtigung der damit verbundenen Chancen und Risiken zu erschließen.

Im Bereich der Technikentwicklung bieten Ansätze der nutzerorientierten und partizipativen Gestaltung dazu einen zielführenden Rahmen im Sinne der inklusiven Forschung. In der Forschungspraxis bestehen dabei aber erhebliche qualitative Unterschiede. Beispiele guter Praxis und Leitfäden für den Umgang mit den Herausforderungen und Widersprüchlichkeiten der partizipativen Technikgestaltung im Kontext von Behinderung und Teilhabe sind bisher rar. Grundsätzlich sind hierbei praxisbasierte Forschungsansätze zu empfehlen, welche eine praktische Nutzung neuer Technologien unter realistischen Anwendungsbedingungen in den Mittelpunkt stellen. Zusätzlich wären für die Zukunft grundlegende Forschungsarbeiten zu methodischen Ansätzen sowie entsprechende Rahmenbedingungen in der Forschungs(förder)praxis wünschenswert.

Zwei Bereiche, denen in der Forschung bisher deutlich zu wenig Aufmerksamkeit gewidmet zu werden scheint, sind die Rolle von Consumertechnik zur Förderung der Teilhabe von Menschen mit Behinderung sowie die Betrachtung von Imple- 
mentierungsbarrieren für den Transfer neuer Technologien in die Versorgungspraxis. Neben der Forschung zur Entwicklung neuer technischer Assistenzsysteme bedarf es hierfür zusätzlicher Forschungsaktivitäten zur Versorgungsforschung sowie zu sozialen Innovationen, welche neue Technologien zur Förderung der Teilhabe in den Blick nehmen.

\section{Literatur}

Bejan, A., Kienzler, R., Wieland, M., Wölfel, M. \& Kunze, C. (2017): Creating Interactive Experiences together with People with Dementia - an Inclusive Design Story. In: Branco, M. (Ed.): Dementia Lab Event. Stories from Design and Research, 9-16. Online unter: https://opus.hs-furtwangen. de/frontdoor/index/index/docId/3048. (Abrufdatum: 23.12.2019).

Borges, L.C., Araujo, M.R., Maciel, C. \& Nunes, E.P. (2016): Participatory design for the development of inclusive educational technologies: A systematic review. In: 2016 IEEE Frontiers in Education Conference (FIE). Online unter: DOI:10.1109/FIE.2016.7757563. (Abrufdatum: 16.03.2020).

Dahlbäck, N., Jönsson, A. \& Ahrenberg, L. (1993): Wizard of Oz studies - why and how. In: Knowledge-based systems 6(4), 258-266.

Doughty, K. (2011): SPAs (smart phone applications) a new form of assistive technology. In: Journal of assistive technologies 5 (2), 88-94.

Farin-Glattacker, E., Kirschning, S., Meyer, T. \& Buschmann-Steinhage, R. (2014): Partizipation an der Forschung - eine Matrix zur Orientierung. Ausschuss „Reha-Forschung“ der Deutschen Vereinigung für Rehabilitation (DVfR) und der Deutschen Gesellschaft für Rehabilitationswissenschaften (DGRW) (Hrsg.). Online unter: http://www.dgrw-online.de/files/matrix_ef_1.pdf. (Abrufdatum: 23.12.2019).

Federici, S., Meloni, F. \& Borsci, S. (2016): The abandonment of assistive technology in Italy: a survey of National Health Service users. In: European journal of physical and rehabilitation medicine $52(4), 516-526$.

Fitzpatrick, G., Huldtgren, A., Malmborg, L., Harley, D. \& Ijsselsteijn, W. (2015): Design for agency, adaptivity and reciprocity: reimagining AAL and telecare agendas. In: V. Wulf, K. Schmidt \& D. Randall (Eds.): Designing Socially Embedded Technologies in the Real-World. London: Springer, 305-338.

Goeke, S. (2016): Entwicklungslinien gemeinsamen Forschens. In: T. Buchner, O. Koenig \& S. Schuppener (Hrsg.): Inklusive Forschung. Gemeinsam mit Menschen mit Lernschwierigkeiten forschen. Bad Heilbrunn: Klinkhardt, 37-53.

Gómez, D.L. (2015): Little arrangements that matter. Rethinking autonomy-enabling innovations for later life. In: Technological Forecasting and Social Change 93, 91-101.

Haage, A. \& Bosse, I. (2019): Basisdaten zur Mediennutzung von Menschen mit Behinderungen. In: I. Bosse, J.-R. Schluchter \& I. Zorn (Hrsg.): Handbuch Inklusion und Medienbildung. Weinheim: Beltz/Juventa, 49-64.

Haage, A. \& Bühler, C. (2019): Barrierefreiheit. In: I. Bosse, J.-R. Schluchter \& I. Zorn (Hrsg.): Handbuch Inklusion und Medienbildung. Weinheim: Beltz Juventa, 207-215.

Haworth, B., Usman, M., Baljko, M. \& Hamidi, F. (2016): The Use of Working Prototypes for Participatory Design with People with Disabilities. In: K. Miesenberger, C. Bühler \& P. Penaz (Eds.): International Conference on Computers Helping People with Special Needs. Cham: Springer, 134-141.

Hutchinson, H. u.a. (2003): Technology probes: inspiring design for and with families. In: G. Cockton \& P. Korhonen (Eds.): Proceedings of the SIGCHI conference on Human factors in computing systems. New York: Association for Computing Machinery, 17-24 
Kensing, F., Blomberg, J. (1998): Participatory design: Issues and concerns. In: Computer Supported Cooperative Work (CSCW) 7 (3), 167-185.

Kunze, C. (2017): Technikgestaltung für die Pflegepraxis. In: Pflege \& Gesellschaft 2017 (2), 130-145.

Kunze, C. (2019): Assistive Technologien in der häuslichen Umgebung. In: C. Dockweiler \& F. Fischer (Hrsg.): ePublic Health. Göttingen: Hogrefe, 199-210.

Kunze, C. (2020): (Nicht-)Nutzung, Transfer, Verbreitung und Nachhaltigkeit von Gesundheitstechnologien: Deutsche Version des NASSS-Frameworks. Hochschule Furtwangen, 2020. Online unter: https://opus.hs-furtwangen.de/frontdoor/index/index/docId/6230. (Abrufdatum: 16.03.2020).

Magnusson C., Hedvall PO. \& Breidegard B. (2018): Design for Me? In: K. Miesenberger \& G. Kouroupetroglou (Eds.): Computers Helping People with Special Needs. ICCHP 2018. Lecture Notes in Computer Science, Vol. 10896. Cham: Springer, 93-99.

Matiouk, S. (2019): Innovation Technologiedesign. In: I. Bosse, J.-R. Schluchter \& I. Zorn (Hrsg.): Handbuch Inklusion und Medienbildung. Weinheim: Beltz Juventa, 359-367.

Mayerle, M. (2015): „Woher hat er die Idee?“: Selbstbestimmte Teilhabe von Menschen mit Lernschwierigkeiten durch Mediennutzung. Abschlussbericht der Begleitforschung im PIKSL-Labor. Siegen: universi - Universitätsverlag Siegen.

Mol, A., Moser, I. \& Pols, J. (2010): Care: putting practice into theory. In: A. Mol, I. Moser \& J. Pols (Eds.): Care in practice: On tinkering in clinics, homes and farms. Bielefeld: transcript, 7-27.

Peine, A., Rollwagen, I. \& Neven, L. (2014): The rise of the „innosumer“ - rethinking older technology users. In: Technological Forecasting and Social Change 82, 199-214.

Rajapakse, S.R.B. (2018): Making collaborations to design personalised technologies with people with an intellectual disability. Doctoral dissertation, Queensland University of Technology. Online unter: DOI:10.5204/thesis.eprints.122963. (Abrufdatum: 16.03.2020).

Wulf, V., Müller, C., Pipek, V., Randall, D., Rohde, M. \& Stevens, G. (2015): Practice-Based Computing: Empirically Grounded Conceptualizations Derived from Design Case Studies. In: V. Wulf, K. Schmidt \& D. Randall (Eds.): Designing Socially Embedded Technologies in the Real-World. London: Springer, 111-150. 
Markus Rebstock

\section{Barrierefreiheit in Planungsprozessen}

\section{Gesetzliche Vorgaben zur Beteiligung von Menschen mit Behinderungen im Planungsprozess am Beispiel der Herstellung von Barrierefreiheit im öffentlichen Verkehrsraum}

Die Herstellung von Barrierefreiheit in den Bereichen Bau und Verkehr wird auf Bundesebene im Gesetz zur Gleichstellung von Menschen mit Behinderungen (Behindertengleichstellungsgesetz BGG) gefordert. So sind gemäß BGG

„sonstige bauliche oder andere Anlagen, öffentliche Wege, Plätze und Straßen sowie öffentlich zugängliche Verkehrsanlagen und Beförderungsmittel im öffentlichen Personenverkehr [...] nach Maßgabe der einschlägigen Rechtsvorschriften des Bundes barrierefrei zu gestalten." (\$ 8 Abs. 5 BGG).

Zeitgleich mit der Einführung des BGG am 1. Mai 2002 trat auch das Gesetz zur Gleichstellung behinderter Menschen und zur Änderung anderer Gesetze (BGGEG) in Kraft. Auf dieser Grundlage wurden rund fünfzig weitere Gesetze an die allgemeine Gesetzeslage angepasst und eine Reihe von Gesetzen im Sinne der Gleichstellung von Menschen mit Behinderungen geändert. Dementsprechend wurden auch diverse Änderungen mit Bezug zum öffentlichen Verkehrsraum vorgenommen. Für den öffentlichen Personennahverkehr (ÖPNV) sind im Hinblick auf die Verankerung der Beteiligung von Menschen mit Behinderungen im Planungsprozess folgende Gesetze von besonderer Bedeutung:

- Personenbeförderungsgesetz

- Gemeindeverkehrsfinanzierungsgesetz

\subsection{Personenbeförderungsgesetz (PBefG)}

Im Bereich des ÖPNV wurde mit Artikel 51 BGGEG das PBefG in $\$ 8$ u.a. um die Pflicht erweitert, möglichst weitreichende Barrierefreiheit im sogenannten Nahverkehrsplan (NVP) zu berücksichtigen sowie bei dessen Aufstellung Beauftragte bzw. Beiräte für Menschen mit Behinderungen anzuhören (vgl. Rebstock 2013, 64). Der entsprechende $₫ 8$ PBefG wurde 2013 präzisiert und mit einer politischen Zielbestimmung ergänzt:

„Der Nahverkehrsplan hat die Belange der in ihrer Mobilität oder sensorisch eingeschränkten Menschen mit dem Ziel zu berücksichtigen, für die Nutzung des öffentlichen 
Personennahverkehrs bis zum 1. Januar 2022 eine vollständige Barrierefreiheit zu erreichen. Die in Satz 3 genannte Frist gilt nicht, sofern in dem Nahverkehrsplan Ausnahmen konkret benannt und begründet werden. Im Nahverkehrsplan werden Aussagen über zeitliche Vorgaben und erforderliche Maßnahmen getroffen. [...] soweit vorhanden sind Behindertenbeauftragte oder Behindertenbeiräte, Verbände der in ihrer Mobilität oder sensorisch eingeschränkten Fahrgäste und Fahrgastverbände anzuhören. Ihre Interessen sind angemessen und diskriminierungsfrei zu berücksichtigen. [...]“ (\$8 Abs. 3 PBefG)

Der NVP ist ein Planungsinstrument für den ÖPNV, mittels dessen eine realistische Planungsgrundlage für die Ausgestaltung des ÖPNV unter Berücksichtigung der zur Verfügung stehenden Finanzmittel und der Tragfähigkeit der festgeschriebenen Maßnahmen geschaffen sowie ein zwischen allen relevanten Akteuren abgestimmtes Vorgehen gewährleistet werden soll. Dieser ist vom ÖPNV-Aufgabenträger aufzustellen, welcher auch die Organisation und Finanzierung des ÖPNV zu verantworten hat. Meist sind dies die Landkreise und kreisfreien Städte, z.T. aber auch Zweckverbände oder Verkehrsverbünde. Mit Ausnahme von Hamburg besteht die Pflicht zur Aufstellung eines NVP in ganz Deutschland (Rebstock 2019, 287).

In Bezug zur Beteiligung der Vertretungen von Menschen mit Behinderungen an der Aufstellung von Nahverkehrsplänen ist festzustellen, dass mit Änderung des PBefG in 2013 die anhörungsberechtigten Interessensvertretungen erweitert wurden. Während vor der PBefG-Änderung nach $\$ 8$ Abs. 3 PBefG lediglich „Behindertenbeauftragte oder Behindertenbeiräte der Aufgabenträger soweit vorhanden anzuhören" (zitiert in Rebstock 2013, 64) waren, sind nun auch Verbände der in ihrer Mobilität oder sensorisch eingeschränkten Fahrgäste und Fahrgastverbände zu beteiligen. Gleichwohl die anhörungsberechtigten Interessenvertretungen immer noch nur dann zu beteiligen sind, sofern diese überhaupt vorhanden sind, steigt durch die Ausweitung der anzuhörenden Interessenvertretungen auch auf zivilgesellschaftliche Organisationen wie Fahrgastverbände dennoch die Wahrscheinlichkeit, dass vor Ort auch tatsächlich anhörungsberechtigte Vertretungen existieren. Insofern ist diese Ausweitung in Bezug auf Beteiligung von Menschen mit Behinderungen im Planungsprozess generell zu begrüßen.

Auf die Änderung der Forderung im PBefG von möglichst weitreichender Barrierefreiheit hin zu vollständiger Barrierefreiheit sowie der Integration der politischen Zielbestimmung bis zum 1. Januar 2022 wird im Folgenden nicht weiter eingegangen, da dies den Fokus des vorliegenden Beitrages übersteigen würde. Diesbezüglich wird auf einschlägige Veröffentlichungen verwiesen (vgl. z.B. Bundesarbeitsgemeinschaft ÖPNV der kommunalen Spitzenverbände 2014, Agentur Barrierefrei NRW am Forschungsinstitut Technologie und Behinderung (FTB) 2016, Rebstock 2017). 


\subsection{Gemeindeverkehrsfinanzierungsgesetz (GVFG)}

Auf Grundlage des GVFG gewährt der Bund den Ländern Finanzhilfen für Investitionen zur Verbesserung der Verkehrsverhältnisse der Gemeinden ( $\$ 1$ GVFG). Diese Finanzhilfen sind sowohl für den öffentlichen Verkehr als auch für den kommunalen Straßenbau einsetzbar. Voraussetzung für eine GVFG-Förderung ist u.a., dass das zu fördernde Vorhaben in einem Generalverkehrsplan oder gleichwertigen Plan vorgesehen sein muss ( $\$ 3$ Nr. 1b GVFG). Daraus folgt, dass wenn gemäß Landesrecht eine Pflicht zur Aufstellung von Nahverkehrsplänen (vgl. Abschnitt 1.1) besteht, das GVFG-Vorhaben auch Bestandteil des NVP sein muss. Relevant für die Herstellung der Barrierefreiheit ist die Fördervoraussetzung nach $\S 3$ Nr. 1d GVFG, die mit Inkrafttreten von Artikel 49 BGGEG präzisiert wurde:

„Voraussetzung für die Förderung nach $₫ 2$ ist, dass [...] das Vorhaben [...] d) Belange behinderter und anderer Menschen mit Mobilitätsbeeinträchtigung berücksichtigt und den Anforderungen der Barrierefreiheit möglichst weitreichend entspricht. Bei der Vorhabenplanung sind die zuständigen Behindertenbeauftragten oder Behindertenbeiräte anzuhören. Verfügt eine Gebietskörperschaft nicht über Behindertenbeauftragte oder Behindertenbeiräte sind stattdessen die entsprechenden Verbände im Sinne des $\$ 5$ des Behindertengleichstellungsgesetzes anzuhören.“ ( $\$ 3 \mathrm{Nr}$. 1d GVFG)

Im Jahr 2006 wurde allerdings durch die sogenannte Föderalismusreform u.a. das GVFG neu gefasst. Dadurch sind die Zweckbindung an die Barrierefreiheit sowie die Beteiligung der Menschen mit Behinderungen an der Vorhabenplanung für die sogenannten Länderprogramme weggefallen (Rebstock 2013, 66). Demnach legen die Bundesländer seit 2007 selbst die Fördervoraussetzungen zur Vergabe dieser Finanzmittel fest. Die meisten Bundesländer haben aber an der vorherigen Verfahrensweise festgehalten und somit auch die Barrierefreiheit weiterhin als eine der Fördervoraussetzungen anerkannt (Verband Deutscher Verkehrsunternehmen 2012, 192). Daneben wurde der Verfahrensablauf z.T. auch optimiert (vgl. hierzu Rebstock 2013, 70f.).

In Bezug zur Beteiligung der Vertretungen von Menschen mit Behinderungen an den Vorhabenplanungen nach GVFG ist im Vergleich zu den Beteiligungsrechten auf Grundlage des PBefG (vgl. Abschnitt 1.1) festzustellen, dass das GVFG die Beteiligung der nach $₫ 15$ Abs. 3 BGG anerkannten Bundesverbände von Menschen mit Behinderungen vorsieht, wenn keine Beauftragten oder Beiräte von Menschen mit Behinderungen im Planungsgebiet vorhanden sind. Dadurch ist, im Gegensatz zur Regelung im PBefG, zumindest gewährleistet, dass bei jedem Vorhaben Interessenvertreter von Seiten des Vorhabenträgers kontaktiert werden können.

\subsection{Gesetzliche und ordnungsrechtliche Regelungen auf Länderebene}

Neben den im Rahmen der Einführung des BGGEG vorgenommenen Anpassungen diverser Bundesgesetze (vgl. Abschnitt 1.1 und 1.2), wurden in den folgenden 
Jahren auch auf Länderebene entsprechende Gesetze verabschiedet. So wurden Landesgesetze zur Gleichstellung von Menschen mit Behinderungen in Kraft gesetzt (vgl. Frehe \& Welti 2018, 104ff.) und auch diverse Länderfachgesetze angepasst. Diesbezüglich fordert z.B. das Thüringer ÖPNV-Gesetz:

„Bei der Planung und Ausgestaltung der Verkehrsinfrastruktur, der Fahrzeugparks sowie des Angebots des ÖPNV sind die Belange von Personen, die in ihrer Mobilität eingeschränkt oder in besonderer Weise auf den ÖPNV angewiesen sind, angemessen zu berücksichtigen." ( $\$ 2$ Abs. 7 ThürÖPNVG)

Daneben sind auf Länderebene weitere ordnungsrechtliche Instrumente für die Barrierefreiheit im öffentlichen Verkehrsraum relevant, so z.B. die Bauordnungen der Länder mit den zugeordneten Verwaltungsvorschriften Technische Baubestimmungen (betrifft i.W. Hochbauvorhaben, z.B. Bahnhofsgebäude) sowie Investitionsrichtlinien für den ÖPNV bzw. kommunalen Straßenbau. Im Gegensatz zum bisher genannten Gesetzesrahmen wird durch diese Instrumente z.T. auch auf deutschlandweit gültige Normen und Regelwerke (vgl. Abschnitt 2) verwiesen bzw. werden diese als technische Regeln für bauliche Anlagen zur Pflichtanwendung eingeführt. Auch kann dadurch eine Planungsbeteiligung („Anhörungsrecht") der Interessenvertretungen von Menschen mit Behinderungen verankert werden (Rebstock 2013, 67).

\section{Normen und Regelwerke zur Barrierefreiheit im öffentlichen Verkehrsraum}

Da die in Abschnitt 1 genannten einschlägigen Gesetze zur Herstellung der Barrierefreiheit im öffentlichen Verkehrsraum keine Vorgaben beinhalten, wie die Barrierefreiheit konkret erreicht werden soll, wurden zur Präzisierung dieses unbestimmten Rechtsbegriffs (vgl. Rebstock 2009, 108 und Bundesministerium für Verkehr, Bau- und Wohnungswesen 2004, 268ff.) diverse deutschlandweit gültige Regelwerke erstellt. So hat der Arbeitskreis 2.14.2 Barrierefreie Verkehrsanlagen der Forschungsgesellschaft für Straßen- und Verkehrswesen e.V. (FGSV) im Jahr 2011 die Hinweise für barrierefreie Verkehrsanlagen (H BVA) als zentrales Regelwerk herausgegeben. Die H BVA behandeln hierbei neben dem Entwurf auch den Prozess zur Planung barrierefreier Verkehrsanlagen. Die H BVA werden derzeit zu Empfehlungen für barrierefreie Verkehrsanlagen weiterentwickelt.

\subsection{DIN-Normen zum barrierefreien Bauen}

Neben Veröffentlichung der H BVA als zentrales Regelwerk der FGSV zur Barrierefreiheit im öffentlichen Verkehrsraum wurden auch die einschlägigen DINNormen des Deutschen Instituts für Normung e.V. zum barrierefreien Bauen 
fortgeschrieben. Die wichtigsten diesbezüglichen Normen, herausgegeben vom DIN-Normenausschuss Bauwesen (NABau), sind:

- DIN 18040 Barrierefreies Bauen - Planungsgrundlagen

- Teil 1: Öffentlich zugängliche Gebäude (DIN 18040-1)

- Teil 2: Wohnungen (DIN 18040-2)

- Teil 3: Öffentlicher Verkehrs- und Freiraum (DIN 18040-3)

Für Aufzugsanlagen bedeutsam ist neben der DIN 18040-1 zudem die vom DINNormenausschuss Maschinenbau (NAM) herausgegebene DIN EN 81-70. Darüber hinaus hat der DIN-Normenausschuss Medizin (NAMed) diverse Begleitnormen veröffentlicht, welche die DIN 18040er Reihe in einzelnen Punkten ergänzen bzw. präzisieren. Zu nennen sind diesbezüglich vor allem:

- DIN 32975: Gestaltung visueller Informationen im öffentlichen Raum zur barrierefreien Nutzung

- DIN 32981: Einrichtungen für blinde und sehbehinderte Menschen an Straßenverkehrs-Signalanlagen (SVA)

- DIN 32984: Bodenindikatoren im öffentlichen Raum (derzeit in Fortschreibung)

- DIN 32986: Taktile Schriften - Anbringung von Braille- und erhabener Profilschrift

Die Anwendung von DIN-Normen ist allerdings nicht unmittelbar verpflichtend. Eine Rechtsverbindlichkeit zur Anwendung kann aber z.B. mittels landesrechtlicher Einführung erfolgen, beispielweise durch Aufnahme in die Verwaltungsvorschrift Technische Baubestimmungen (Rebstock 2013, 68; vgl. Abschnitt 1.3).

\subsection{Performance-Prinzip der Normen-Reihe DIN-18040}

Die DIN 18040 arbeitet nach dem sogenannten Performance-Prinzip, „d.h. es werden „Schutzziele“ definiert und „Beispiellösungen“ benannt.“ (Degenhart u.a. 2013, 4) Dementsprechend haben alle drei Normenteile in ihrem jeweiligen Anwendungsbereich einen diesbezüglichen Hinweis: „Die mit den Anforderungen nach dieser Norm verfolgten Schutzziele können auch auf andere Weise als in der Norm festgelegt erfüllt werden." (DIN 18040-1, 4; DIN 18040-2, 4 und DIN 18040-3, 5)

Im jeweiligen Abschnitt dieser Normen werden daher i.d.R. zuerst die zu erfüllenden Schutzziele als Voraussetzung für die Erreichung von Barrierefreiheit aufgeführt. Im Anschluss wird dargelegt, wie das jeweilige Schutzziel erreicht werden kann. Zum Teil werden die Lösungsmöglichkeiten auch nach den unterschiedlichen Bedürfnissen verschiedener Personengruppen differenziert. „Es ist daher immer zulässig, das Ziel auch mit einer anderen Lösung zu erreichen. Insbesondere technische Neuerungen können zu weiteren Lösungsmöglichkeiten führen (Performance-Prinzip).“ (Degenhart u.a. 2013, 18) 
Auch die H BVA arbeiten mit einer Art von Schutzzielformulierung. So kann das Ziel einer barrierefreien Verkehrsanlage gemäß H BVA prinzipiell auf verschiedenen Wegen erreicht werden, wenn folgende drei Bedingungen erfüllt sind (Forschungsgesellschaft für Straßen- und Verkehrswesen e.V. - Arbeitsgruppe Straßenentwurf 2011, 7):

- Gestaltungs- und Bauvarianten sind auf lokaler Ebene bereits seit längerer Zeit eingeführt

- Gestaltungs- und Bauvarianten erfüllen auch heute noch nachweislich ihren funktionalen Zweck

- Gestaltungs- und Bauvarianten stellen von Seiten der Nutzenden eine akzeptierte Lösung dar

Daneben sieht auch das BGG Schutzziele für Bundesbauten vor:

„Zivile Neu-, Um- und Erweiterungsbauten im Eigentum des Bundes einschließlich der bundesunmittelbaren Körperschaften, Anstalten und Stiftungen des öffentlichen Rechts sollen entsprechend den allgemein anerkannten Regeln der Technik barrierefrei gestaltet werden. Von diesen Anforderungen kann abgewichen werden, wenn mit einer anderen Lösung in gleichem Maße die Anforderungen an die Barrierefreiheit erfüllt werden." (\$8 Abs.1 BGG)

Folglich sind Abweichungen von den Norm- bzw. Regelwerksvorgaben grundsätzlich möglich, wenn das Schutzziel bzw. die Bedingungen der H BVA erfüllt sind. Diese prinzipiell vernünftige Herangehensweise zur Ermöglichung von Innovationen und Belebung der Kreativität der Planenden führt in der Planungspraxis allerdings durchaus zu Problemen. So enthalten z.B. die einschlägigen DIN-Normen der 329er-Reihe zur Barrierefreiheit keine Schutzziele, was anhand von konkreten Planungsvorhaben zu Meinungsverschiedenheiten zwischen den an der Planung Beteiligten führen kann, insbesondere wenn Interessenvertretungen auf der strikten Einhaltung von Normteilen der 329er-Reihe bestehen, die verantwortlichen Planenden aber eine Lösung im Sinne der Schutzziele befürworten. Generell obliegt es allerdings dem Planenden ,im Zweifelsfall zu belegen, dass die von ihm angestrebte oder realisierte Lösung das Schutzziel ebenfalls erfüllt." (Rebstock \& Sieger 2015, 8) Im öffentlichen Verkehrs- und Freiraum wird dieser potentielle Dissens zwischen den unterschiedlichen Normenreihen durch eine zusätzliche Abwägungsklausel im Anwendungsbereich der DIN 18040-3 noch verstärkt:

„Die Norm definiert die Nutzungsansprüche für einen barrierefreien Fußgängerverkehr. Sie geht davon aus, dass im öffentlichen Verkehrs- und Freiraum konkurrierende Nutzungsansprüche auftreten können, die im Rahmen des Planungsprozesses untereinander abgewogen werden." (DIN 18040-3, 5)

Diese vom Grundsatz für den komplexen öffentlichen Verkehrs- und Freiraum fachgerechte und somit angemessene Abwägungsklausel sowie die im Detail nicht 
immer widerspruchsfreien Regelungen innerhalb der verschiedenen Normen und Regelwerke können aber im Rahmen von Planungsbeteiligungen zu schwierigen Diskussionen und unauflösbaren Dissensen führen. Um diese Problematik zu entschärfen, muss neben einer Weiterentwicklung der Normen und Regelwerke im Hinblick auf Widerspruchsfreiheit auch die Hierarchie der Regelwerke und Normen untereinander geklärt werden. Die Normen des NABau (vgl. Abschnitt 2.1) sollten dabei als übergeordnete Planungsnormen die Grundlage bilden, unter die sich alle weiteren Normen einzuordnen haben. Dementsprechend müssen Normen zur Barrierefreiheit, die Bereiche des öffentlichen Verkehrs- und Freiraumes berühren, das übergeordnete Regelwerk inklusive des dort verankerten Performance-Prinzips konsequent respektieren und dürfen in ihren Regelungen nicht abweichen oder darüber hinausgehen.

\section{Grenzen der Beteiligung}

Wie in Abschnitt 1 dargestellt, ist eine Beteiligung von Vertretungen von Menschen mit Behinderungen an Planungsprozessen zur Barrierefreiheit im öffentlichen Verkehrsraum bereits in den einschlägigen Gesetzen verankert. Allerdings unterscheiden sich die anzuhörenden Vertretungen je nach Gesetz und die Anhörung ist z.T. auch unter den Vorbehalt gestellt, ob bestimmte Vertretungen vor Ort existieren oder nicht. In der Planungspraxis werden zudem über die nach Gesetzeslage eigentlich anzuhörenden Vertretungen hinaus oftmals noch weitere Personen beteiligt. Erfahrungen zeigen, dass

- meistens die zuständigen Beauftragten oder Beiräte für Menschen mit Behinderungen angehört werden,

- z.T. auch die nach $\$ 15$ Abs. 3 BGG anerkannten Verbände von Menschen mit Behinderungen beteiligt werden und

- oftmals zusätzlich lokal aktive Verbände, Vereine und Bürger mit Behinderungen einbezogen werden.

Indes vertreten auch Selbsthilfevereinigungen von Menschen mit Behinderungen insbesondere auf lokaler Planungsebene z.T. keine einheitlichen Lösungen. Darüber hinaus haben Vorhabenträger, Planende, Stadtgestaltende, Denkmalschützende und Beauftragte für Menschen mit Behinderungen oftmals unterschiedlichste Vorstellungen über die konkrete Umsetzung der Barrierefreiheit. Dies führt mitunter dazu, dass unterschiedlichste Lösungsansätze umgesetzt werden, die zu einer nicht mehr überschaubaren Vielfalt geführt haben (vgl. Rebstock 2009, 133ff.). Um diese Vielfalt einzugrenzen ,sollten nur noch die aktuellen Regelwerke [(vgl. Abschnitt 2)] bzw. die auf die dort genannten Standards aufbauenden Planungshilfen genutzt werden." (Rebstock 2013, 68) 


\subsection{Werden alle relevanten Gruppen normativ berücksichtigt?}

Primäre Zielgruppen der Normung zur Barrierefreiheit im öffentlichen Verkehrsraum sind (DIN 18040-3, 4):

- Menschen mit sensorischen Einschränkungen wie Sehbehinderung, Blindheit, Hörbehinderung (gehörlose, ertaubte und schwerhörige Menschen)

- Menschen mit motorischen Einschränkungen sowie von Personen, die Mobilitätshilfen und Rollstühle benutzen.

Diese Gruppen werden auch als Menschen mit Mobilitätsbehinderungen im engeren Sinne bezeichnet, welche einem Bevölkerungsanteil von etwa $10 \%$ entsprechen (vgl. Rebstock 2009, 45). Daneben profitieren auch weitere Personengruppen von Barrierefreiheit, wie z.B. groß- oder kleinwüchsige Menschen, Menschen mit altersbedingten oder temporären Beeinträchtigungen, schwangere Frauen, Kinder sowie Personen mit Kinderwagen, Gepäck oder Hunden. Diese werden auch als Menschen mit Mobilitätsbehinderungen im weiteren Sinne bezeichnet und entsprechen einem Bevölkerungsanteil zwischen $20 \%$ und $40 \%$ (Becker u.a. 2007, 581). Darüber hinaus kommt ein barrierefreier Verkehrsraum auch allen anderen zu Fuß Gehenden zu Gute, da dadurch i.d.R. der Komfort erhöht wird (Rebstock 2019, 288), z.B. durch Entfernung von Stufen oder unebenen Oberflächenbelägen.

Bisher nicht normativ berücksichtigt sind allerdings:

\section{- Personen mit kognitiven Einschränkungen}

Obwohl in DIN 18040-3 festgestellt wird, dass „auch für andere Personengruppen, wie z.B. [...] Personen mit kognitiven Einschränkungen [...] einige Anforderungen dieser Norm zu einer Nutzungserleichterung [führen]" (DIN 18040-3, 4), werden keine spezifischen Anforderungen an den öffentlichen Verkehrs- und Freiraum für diese Personengruppe festgelegt. So wird im Kapitel Hinweise zu Wahrnehmungsarten lediglich als Schutzziel formuliert: „Informationen und Orientierungshilfen müssen grundsätzlich auch für Menschen mit kognitiven Beeinträchtigungen leicht begreifbar und gut merkbar sein." (DIN 18040-3, 12) Dabei macht der Begriff grundsätzlich „deutlich, dass die Normgeber dies als allgemeinen Grundsatz verstanden wissen wollen, von dem es naturgemäß Ausnahmen geben wird bzw. von dem abgewichen werden kann." (Rebstock \& Sieger 2015, 52) Auch die im Anschluss aufgeführten Hinweise zur Wahrnehmung in Bezug zu Personen mit kognitiven Einschränkungen werden nur beispielhaft gegeben. Grund dafür ist, dass

„in Bezug auf die entscheidenden Einflussfaktoren, was das Orientieren im öffentlichen Raum und seine selbständige Nutzung angeht, sowie damit einhergehende Anforderungen an die Barrierefreiheit [...] bislang kaum belastbare Forschungsergebnisse vor[liegen], die zugleich die Heterogenität der betroffenen Personengruppe angemessen berücksichtigen." (Rebstock \& Sieger 2015, 52) 
Demzufolge ist zunächst die Generierung von entsprechendem Fachwissen erforderlich, um die Belange von Personen mit kognitiven Einschränkungen künftig im Normungsverfahren fachgerecht berücksichtigen zu können.

\section{- Personen mit Mehrfachbehinderungen}

„Trotz aller Bestrebungen, die Umwelt [...] allen Menschen zugänglich zu machen, ist dies leider nicht möglich. DIN 18040 [...] bildet die Bedürfnisse einer sehr großen Anzahl von Nutzern ab, dennoch werden immer einige Nutzer auf zusätzliche Maßnahmen oder Assistenz angewiesen bleiben." (Loeschcke u.a. 2010, 35)

So berücksichtigt die Normung zur Barrierefreiheit im öffentlichen Verkehrsraum zwar die o.g. verschiedenen Behinderungsarten, allerdings nicht in Form von Mehrfachbehinderungen. Beispielsweise werden Personen mit Taubblindheit oder mit einer Körper- und einer Sinnesbehinderung normativ nicht erfasst.

- Nutzende von Rollstühlen und Mobilitätshilfen, deren Rollstuhlmaße die Vorgaben der DIN EN 12183 bzw. DIN EN 12184 für Rollstühle für den Innen- und Außenbereich übersteigen

DIN 18040-3 leitet den Flächen- und Raumbedarf im öffentlichen Verkehrsund Freiraum von denjenigen Personen ab, „die je nach Situation den größten Flächenbedarf haben, in der Regel Nutzer von Rollstühlen, Gehhilfen oder Langstöcken." (DIN 18040-3, 8) Dabei liegen der Flächenermittlung eines Rollstuhlnutzenden die internationalen Normen DIN EN 12183 und DIN EN 12184 zugrunde.

„Damit werden nahezu alle nicht motorisierten Standardrollstühle und ein Großteil der Elektrorollstühle, die sowohl für den Einsatz im Innen- als auch im Außenbereich vorgesehen sind, erfasst. Unberücksichtigt bleiben allerdings individuell auf die Beeinträchtigungen eines speziellen Nutzers hin oder aufgrund einer bestimmten Funktion (z.B. Sport) angefertigte Rollstühle mit größeren Abmessungen sowie solche Elektrorollstühle, die vornehmlich für den Außenbereich konzipiert sind." (Rebstock \& Sieger 2015, 23)

\subsection{In welcher Form sollen Planungsunterlagen im Rahmen von}

Beteiligungsverfahren barrierefrei aufbereitet werden?

Neben den o.g. gesetzlich vorgegebenen Grenzen der Beteiligung sowie der normativen Grenzen gibt es in der Planungspraxis noch weitere Punkte, die zur Verunsicherung auf Seiten der Vorhabenträger im Hinblick auf die Beteiligung von Menschen mit Behinderungen am Planungsprozess führen können. Beispielhaft werden an dieser Stelle folgende Fragen genannt:

- Bei welchen Planungsvorhaben ist welche barrierefreie Übermittlungs- und Darstellungsmethode angemessen?

In Abhängigkeit vom Volumen und der Komplexität eines Vorhabens muss im Einzelfall entschieden werden, für welche Zielgruppen welche barrierefreien 
Darbietungsformen gewählt werden. Der Bedarf sollte generell zielgerichtet im Vorfeld abgefragt werden, um keine vermeidbaren Kosten zu verursachen.

- Sind taktile Planunterlagen überhaupt „lesbar“?

Eine Evaluierung der Praktikabilität von taktilen Planunterlagen im Hinblick auf deren Begreifbarkeit vor dem Hintergrund von Aufwand und Nutzen wäre anzuraten. Aus den Ergebnissen könnte ein System entwickelt werden, mit welchem künftig abgeleitet werden kann, in welchen Fällen taktile Planunterlagen zielführend sind.

- Ist es sinnvoll, Planungen in „Leichte Sprache“ zu übersetzen?

Derzeit ist zu beobachten, dass immer mehr Publikationen insbesondere von öffentlichen Stellen zusätzlich auch in sogenannter Leichter Sprache veröffentlicht werden. Bisher nicht untersucht sind generell die tatsächliche Nutzung dieser Veröffentlichungen durch die Zielgruppen sowie im Speziellen die Sinnhaftigkeit der Übersetzung von Planunterlagen in Leichte Sprache.

- Müssen zusätzlich Pläne erstellt werden, die den Anforderungen an die visuelle Kontrastgestaltung entsprechen?

Planunterlagen sind oftmals in ihrer Darstellung kontrastarm gestaltet. Dies verhindert deren Erkennbarkeit durch seheingeschränkte Personen im Zuge von Planungsbeteiligungen. Ungeklärt ist die Frage, ob in diesen Fällen von den Planenden visuell kontrastreich gestaltete Planunterlagen gefordert werden können bzw. müssen oder ob verbale Erläuterungen im Zuge der Anhörungsverfahren ausreichen.

- Sind Gebärdensprach-, Schriftdolmetscher, Hörschleifen usw. erforderlich? Im Einzelfall sind bei konkretem Bedarf entsprechende Hilfsmittel für die direkte Kommunikation zwischen Vorhabenträger, Planenden und Anzuhörenden bereit zu stellen. Für gehörlose Personen sind z.B. Gebärdensprach- und/ oder Schriftdolmetscher vorzuhalten, für schwerhörige Personen z.B. induktive Hörschleifen.

\section{Fazit}

Die Beteiligung von Menschen mit Behinderungen im Rahmen von Planungsprozessen in den Bereichen Bau und Verkehr ist weitgehend gesetzlich verankert. Gleichwohl unterscheidet sich der Kreis der Anzuhörenden in Abhängigkeit von der gesetzlichen Grundlage und der tatsächlichen Verfügbarkeit potentiell anzuhörender Vertretungen vor Ort. In den überwiegenden Fällen sind allerdings die kommunalen Beauftragten für Menschen mit Behinderungen im Planungsprozess zu beteiligen. Voraussetzung dafür ist, dass überhaupt eine entsprechende Vertretung im Planungsgebiet berufen wurde. Dementsprechend bedeutsam ist die Rolle dieser Beauftragten im Planungsprozess, die Einrichtung eines entspre- 
chenden Amtes auf kommunaler Ebene ist daher anzuraten. Bei der Ausgestaltung dieses Amtes ist zudem sicherzustellen, dass sich die Beauftragten für Menschen mit Behinderungen die notwendigen Qualifikationen hinsichtlich der Gestaltung barrierefreier öffentlicher Verkehrssysteme aneignen können und angemessene Zeitbudgets zur Ausübung ihres Amtes zur Verfügung haben. Folglich ist den Beauftragten für die Erwerbung des notwendigen Fachwissens ein angemessenes finanzielles Budget für die eigene fachliche Fortbildung bzw. bei Erfordernis auch für die Hinzuziehung externer Beratungsleistungen zur Verfügung zu stellen (vgl. hierzu auch Rebstock 2009, 134ff.).

Bezüglich der Anwendung bestehender deutschlandweit gültiger Regelwerke zur Barrierefreiheit ist darauf zu achten, dass nur die aktuellen Regelwerke bzw. die auf die dort genannten Standards aufbauenden Planungshilfen angewandt werden. Daneben sollte im Rahmen der Weiterentwicklung der Normen zur Barrierefreiheit, neben der fortlaufenden Beseitigung von Widersprüchen zwischen den Normen, verstärkt darauf geachtet werden, die Normhierarchie klarer zu strukturieren.

Unsicherheiten von Seiten der Vorhabenträger und Planenden können in Bezug auf die Wahl von sachgerechten Formen barrierefreier Übermittlungs- und Darstellungsmethoden im Rahmen von Planungsvorhaben entstehen: Sind grundsätzlich bzw. bei welchen Vorhaben sind taktile und/oder visuell kontrastierende Planunterlagen und/oder Unterlagen in Leichter Sprache erforderlich? Braucht es spezielle Hilfsmittel für die Kommunikation im Anhörungsverfahren, wie Gebärdensprach-, Schriftdolmetscher, Hörschleifen usw.? Diesbezüglich kann keine allgemeingültige Vorgabe formuliert werden. Letztlich muss dies im Einzelfall anhand der Planungsaufgabe und dem tatsächlichen Kreis der anzuhörenden Vertretungen entschieden werden. Um keine unnötigen Kosten zu verursachen, wird gleichwohl empfohlen, den konkreten Bedarf der barrierefreien Darbietungsformen im Vorfeld zielgerichtet zu ermitteln.

\section{Literatur}

Agentur Barrierefrei NRW am Forschungsinstitut Technologie und Behinderung (FTB) (2016): Definition „Vollständige Barrierefreiheit im öffentlichen Personennahverkehr zum novellierten Personenbeförderungsgesetz (PBefG). (Hrsg.): Die Beauftragte der Landesregierung für die Belange der Menschen mit Behinderung in Nordrhein-Westfalen. Düsseldorf, Wetter/Ruhr. Online unter http://pbefg.ab-nrw.de/. (Abrufdatum: 10.12.2019).

Becker, J., Schubert, S., Vollmer, P.E. \& Wahlster, M.N. (2007): Schwerbehinderung und Mobilitätseinschränkung. In: Internationales Verkehrswesen: Fachzeitschrift für Wissenschaft und Praxis 59(12), S. 580-584.

Bundesarbeitsgemeinschaft ÖPNV der kommunalen Spitzenverbände (2014): Vollständige Barrierefreiheit im ÖPNV. Hinweise für die ÖPNV-Aufgabenträger zum Umgang mit der Zielbestimmung des novellierten PBefG, erarbeitet durch eine ad-hoc-Arbeitsgruppe der BAG ÖPNV. Chemnitz (u.a.). 
Bundesministerium für Verkehr, Bau- und Wohnungswesen (Hrsg.) (2004): Auswirkungen des Gesetzes zur Gleichstellung behinderter Menschen (BGG) und zur Änderung anderer Gesetze auf die Bereiche Bau und Verkehr. FE 70.0703/2000. Köln, Mainz.

Degenhart, Ch., Ebe, J. \& Famers, G. (2013): Barrierefreies Bauen 01 öffentlich zugängliche Gebäude. Planungsgrundlagen. (Hrsg.): Bayerische Architektenkammer, Oberste Baubehörde im Bayerischen Staatsministerium des Innern und Bayerisches Staatsministerium für Arbeit und Sozialordnung, Familie, Frauen. München.

DIN 18040-1 - Deutsches Institut für Normung e.V.: Barrierefreies Bauen - Planungsgrundlagen Teil 1: Öffentlich zugängliche Gebäude, Oktober 2010, Berlin.

DIN 18040-2 - Deutsches Institut für Normung e.V.: Barrierefreies Bauen — Planungsgrundlagen — Teil 2: Wohnungen, September 2011, Berlin.

DIN 18040-3 - Deutsches Institut für Normung e.V.: Barrierefreies Bauen — Planungsgrundlagen Teil 3: Öffentlicher Verkehrs- und Freiraum, Dezember 2014, Berlin.

DIN 32975 - Deutsches Institut für Normung e.V.: Gestaltung visueller Informationen im öffentlichen Raum zur barrierefreien Nutzung, November 2009, Berlin.

DIN 32981 - Deutsches Institut für Normung e.V.: Einrichtungen für blinde und sehbehinderte Menschen an Straßenverkehrs-Signalanlagen (SVA) - Anforderungen, Oktober 2015, Berlin.

DIN 32984 - Deutsches Institut für Normung e.V.: Bodenindikatoren im öffentlichen Raum, Oktober 2011, Berlin.

DIN 32986 - Deutsches Institut für Normung e.V.: Taktile Schriften - Anbringung von Braille- und erhabener Profilschrift, Januar 2015, Berlin.

DIN EN 12183 - Deutsches Institut für Normung e.V.: Rollstühle mit Muskelkraftantrieb - Anforderungen und Prüfverfahren, Dezember 2009, Berlin.

DIN EN 12184 - Deutsches Institut für Normung e.V.: Elektrorollstühle und -mobile und zugehörige Ladegeräte - Anforderungen und Prüfverfahren, Dezember 2009, Berlin.

DIN EN 81-70 - Deutsches Institut für Normung e.V.: Sicherheitsregeln für die Konstruktion und den Einbau von Aufzügen - Besondere Anwendungen für Personen und Lastenaufzüge - Teil 70: Zugänglichkeit von Aufzügen für Personen einschließlich Personen mit Behinderungen, Juli 2018, Berlin.

Forschungsgesellschaft für Straßen- und Verkehrswesen e.V. - Arbeitsgruppe Straßenentwurf (Hrsg.) (2011): Hinweise für barrierefreie Verkehrsanlagen. H BVA. Köln (FGSV, 212).

Frehe, H. \& Welti, F. (Hrsg.) (2018): Behindertengleichstellungsrecht. 3. Aufl. Baden-Baden: Nomos (NomosGesetze).

GVFG (1988): Gemeindeverkehrsfinanzierungsgesetz in der Fassung der Bekanntmachung vom 28. Januar 1988 (BGBl. I S. 100), das zuletzt durch Artikel 463 der Verordnung vom 31. August 2015 (BGBl. I S. 1474) geändert worden ist.

BGG (2002): Gesetz zur Gleichstellung behinderter Menschen (Behindertengleichstellungsgesetz vom 27. April 2002 (BGBl. I S. 1467, 1468), das zuletzt durch Artikel 3 des Gesetzes vom 10. Juli 2018 (BGBl. I S. 1117) geändert worden ist).

BGGEG (2002): Gesetz zur Gleichstellung behinderter Menschen und zur Änderung anderer Gesetze (Behindertengleichstellungs-Einführungsgesetz).

Loeschcke, G., Pourat, D. \& Marx, L. (2010): Barrierefreies Bauen - Band 1. Kommentar zur DIN 18040-1. 1. Auflage. Berlin (Beuth-Kommentar).

PBefG (1990): Personenbeförderungsgesetz in der Fassung der Bekanntmachung vom 8. August 1990 (BGBl. I S. 1690), das zuletzt durch Artikel 2 Absatz 14 des Gesetzes vom 20. Juli 2017 (BGBl. I S. 2808) geändert worden ist.

Rebstock, M. (2019): Öffentliche Mobilität/ÖPNV. In: F. Ross, M. Rund \& J. Steinhaußen (Hrsg.): Alternde Gesellschaften gerecht gestalten. Stichwörter für die partizipative Praxis. 1. Auflage. Opladen, Berlin, Toronto: Verlag Barbara Budrich, S. 287-296.

Rebstock, M. (2017): Vollständige Barrierefreiheit im ÖPNV bis 2022. In: Verkehr und Technik 70 (11.17), S. 383-389. 
Rebstock, M. (2013): Barrierefreiheit in der Planungspraxis. Optimierungspotentiale in Bezug zur Novellierung des BGG am Beispiel des ÖPNV. In: F. Welti (Hrsg.): Rechtliche Instrumente zur Durchsetzung der Barrierefreiheit. Kassel: Kassel University Press, S. 63-72.

Rebstock, M. (2009): Instrumente zur Umsetzung der Barrierefreiheit im öffentlichen Personennahverkehr. Fallstudie zur Anwendbarkeit in ländlich geprägten Tourismusregionen. Zugl.: Trier, Univ., Diss. Tönning: Der Andere Verl. - Universität Trier.

Rebstock, M. \& Sieger, V. (2015): Barrierefreies Bauen. Band 3: Öffentlicher Verkehrs- und Freiraum - Kommentar zu DIN 18040-3. 1. Aufl. Berlin: Beuth (Beuth-Kommentar).

ThürÖPNVG (2005): Thüringer Gesetz über den öffentlichen Personennahverkehr in der Fassung der Bekanntmachung vom 22. Juni 2005, zuletzt geändert durch Artikel 46 des Gesetzes vom 18. Dezember 2018 (GVBl. S. 731, 764).

Verband Deutscher Verkehrsunternehmen (Hrsg.) (2012): Barrierefreier ÖPNV in Deutschland. Barrier-free public transport in Germany. 2. Aufl. Meerbusch: Alba-Fachverl. 



\section{Autorinnen und Autoren}

\section{Friedrich Dieckmann, Prof. Dr., Dipl. Psych.}

Katholische Hochschule Nordrhein-Westfalen

Institut für Teilhabeforschung

Arbeits- und Forschungsschwerpunkte: Heilpädagogische Psychologie, Sozialraumorientiertes Wohnen von Menschen mit intellektueller Beeinträchtigung, Sozialplanung, Alter von Menschen mit lebenslanger Beeinträchtigung, Angehörige von Menschen mit intellektueller Beeinträchtigung

Piusallee 89, 48147 Münster

f.dieckmann@katho-nrw.de

\section{Marianne Hirschberg, Prof. Dr.}

Universität Kassel

Arbeits- und Forschungsschwerpunkte: Menschenrechte, Disability Studies, Teilhabe behinderter Menschen

Arnold-Bode-Str. 10, 34109 Kassel

hirschberg@uni-kassel.de

\section{Leonora Micah Jordan, Dipl. Soz.Arb./-päd., M.A.}

Universität Kassel

Arbeits- und Forschungsschwerpunkte: Beratungskompetenzforschung, Professionalisierung von Peer-Beratung, Konzeption inklusiver Fort- und Weiterbildungsangebote, Pädagogische Beratung

Wiss. Mitarbeiterin und Doktorandin, Trainerin \& Coach für

Teilhabeberater*innen $\left(\mathrm{EUTB}^{\odot}\right)$, Trainerin für Peer Counseling, Playbacktheater Practitioner, Peer Counselor

Arnold-Bode-Straße 10, 34109 Kassel

micah.jordan@uni-kassel.de 


\section{Barbara Klein, Prof. Dr.}

Studiengangsleiterin Inclusive Design - Digital Health und Case Management, Sprecherin des Forschungszentrums FUTURE AGING, Koordinatorin der Ausstellung „Hallo Freiheit! Zusammen über Barrieren“ und des Innovation Labs 5.0; Gastprofessorin der Osaka-University, Japan

Frankfurt University of Applied Sciences

Arbeits- und Forschungsschwerpunkte: Assistive Technologien und Robotik im Healthcare Sektor, Akzeptanz und ethische Fragen Assistiver Technologien, Entwicklung von digitalen Versorgungsstrukturen

Nibelungenplatz 1, 60318 Frankfurt am Main

bklein@fb4.fra-uas.de

Christophe Kunze, Prof. Dr.

Hochschule Furtwangen, Institut Mensch, Technik, Teilhabe (IMTT)

Arbeits- und Forschungsschwerpunkte: Nutzerzentrierte und partizipative Technikgestaltung, Digitalisierung in der Pflege, Digitalisierung und Teilhabe.

Robert-Gerwig-Platz 1, 78120 Furtwangen

kuc@hs-furtwangen.de

Markus Rebstock, Dr.

Institut Verkehr und Raum der Fachhochschule Erfurt

Arbeits- und Forschungsschwerpunkte: Barrierefreiheit im öffentlichen Verkehrsund Freiraum, Hochbau sowie Tourismus; Leiter des AK 2.14.2 Barrierefreie Verkehrsanlagen der FGSV

Postfach 4501 55, 99051 Erfurt

rebstock@fh-erfurt.de

Dominik Rupprecht, M.Sc. Dipl.-Inf. (FH)

Hochschule Fulda

Arbeits- und Forschungsschwerpunkte: Mensch-Computer-Interaktion, Usability, modellbasierte Entwicklung interaktiver Systeme

Leipziger Straße 123, 36037 Fulda

dominik.rupprecht@informatik.hs-fulda.de

\section{Viviane Schachler, M.A. Soziale Arbeit}

Hochschule Fulda

Arbeits- und Forschungsschwerpunkte: Partizipation und Selbsthilfe bei Behinderung/chronischer Erkrankung, betriebliche Mitbestimmung, beeinträchtigungssensible Forschungsmethoden und -ansätze

Leipziger Straße 123, 36037 Fulda

viviane.schachler@sw.hs-fulda.de 
Markus Schäfers, Prof. Dr.

Hochschule Fulda

Arbeits- und Forschungsschwerpunkte: Profilierung einer Teilhabeforschung, Lebensqualitätsforschung/Soziale Indikatoren, Methodenforschung zur Befragung Leipziger Straße 123, 36037 Fulda

markus.schaefers@sw.hs-fulda.de

Tanja Freifrau Schenck zu Schweinsberg (geb. Lück), M.A. Soziale Arbeit Hochschule Fulda

Arbeits- und Forschungsschwerpunkte: Kommunikative Barrierefreiheit \& Teilhabe bei komplexer Behinderung, Unterstützte Kommunikation, Interviewmethode

Leipziger Straße 123, 36037 Fulda

tanja.schenckzuschweinsberg@sw.hs-fulda.de

Matthias Schmidt-Ohlemann, Dr. med.

Deutsche Vereinigung für Rehabilitation (DVfR), Landesarzt für Körperbehinderte in Rheinland-Pfalz

Pestalozzistraße 5, 55543 Bad Kreuznach

Matthias.Schmidt.Ohlemann@googlemail.com

\section{Oliver Sträter, Prof. Dr. habil.}

Universität Kassel, Fachbereich Maschinenbau, Arbeits- und Organisationspsychologie

Arbeits- und Forschungsschwerpunkte: Ergonomie, Organisationsgestaltung und Sicherheit

Heinrich-Plett-Strasse 40, 34132 Kassel

straeter@uni-kassel.de

\section{Felix Welti, Prof. Dr.}

Universität Kassel

Arbeits- und Forschungsschwerpunkte: Sozial- und Gesundheitsrecht, Recht der Rehabilitation und Behinderung

Arnold-Bode-Straße 10, 34109 Kassel

welti@uni-kassel.de 


\section{Beschreibung für Abbildungen und Tabellen}

\section{Oliver Sträter}

\section{Universal Design - Gestaltung der Zugänglichkeit von Arbeitssystemen für Menschen mit Behinderung}

Tab. 1: Analyse der Passung von Tätigkeit und funktionalen Eigenschaften

In der Tabelle werden auf der Horizontalen funktionale Einschränkungen und in der Vertikalen Tätigkeiten aufgetragen. In jeder Zelle finden sich Zahlenwerte. Es ergeben sich unterschiedliche Kombinationen von Einschränkungen und Tätigkeiten, die daraufhin hinsichtlich ihrer Bedeutung für die Arbeitsdurchführung bewertet werden. Die Zellen der Tabelle sind unterschiedlich schattiert: Grau bedeutet „keinerlei Einfluss“, mittelgrau bedeutet „Einfluss, aber durch Gestaltungsmaßnahmen kompensierbar“ und dunkelgrau bedeutet „Tätigkeit mit der Einschränkung nicht möglich“.

Abb. 1: Perzentilierung als methodisches Vorgehen zur Bestimmung von Gestaltungskriterien

Die Abbildung zeigt eine Kurve mit einer Normalverteilung. Am rechten und linken Rand, wo die Kurve jeweils abflacht, finden sich die dargestellten Extreme, in denen eine signifikante Abweichung von der Population zu finden ist. Die Kurve wird links und rechts durch senkrechte Striche abgeschnitten, welche die Extrembereiche kennzeichnen. In der Abbildung sind diese mit x \% dargestellt. Die Extrembereiche werden bei unterschiedlichen Gestaltungskriterien unterschiedlich groß gewählt. Gestaltungslösungen lassen diese Extrembereiche außen vor und fokussieren sich auf den dargestellten mittleren Bereich.

Abb. 2: Vergleich klassischer versus proaktiver Systemgestaltung mit Hilfe eines ScreeningAnsatzes (in Anlehnung an Sträter u.a. 2012)

Die Abbildung stellt durch Flussdiagramme zwei unterschiedliche Prozesse zur Systemgestaltung dar. Die klassische Systemgestaltung wie auf der linken Seite dargestellt geht von einer technischen Machbarkeit aus, die dann in ein Design und eine Entwicklung überführt und darauf pilotiert und getestet wird. Die Implementierung im betrieblichen Kontext führt dann gegebenenfalls zur Modifikation im Design und der Entwicklung. Als letzter Schritt wird die Gestaltungslösung dann angewendet. Auf der rechten Seite wird eine proaktive Systemgestaltung gezeigt. Diese geht nicht von der Machbarkeit, sondern von der funktionalen Idee aus. Der betriebliche Kontext wird daraufhin analysiert hinsichtlich der Idee, die dann in einem engen Optimierungszyklus mit der integrativen Konzeptionierung zu einer technischen Gestaltungslösung entwickelt wird. Hieraus resultiert dann die Anwendung des Systems.

Abb. 3: Hauptkategorien des Screening-Tools

Die Abbildung zeigt die Kategorien des Screening Tools. Sie sind untergliedert in vier Hauptfragen mit vier Unterfragen. Die vier Hauptfragen sind: Betriebliches Management, Arbeitssystem Anwendungsebene und Organisationsebene. Die Unterfragen zum betrieb- 
lichen Management sind: Definierte Abläufe, Produktionsprozess, Qualifizierung/Ausbildung sowie Verantwortlichkeiten. Die Unterfragen zum Arbeitssystem sind: Tätigkeitsgestaltung und Handlungsspielraum, Ergonomie, digitale Werkerassistenz sowie technische Ausstattung/Hilfsmittel. Die Unterfragen zur Anwendungsebene sind: Lernen und Verbesserung, Zusammenarbeit, Kommunikation und Informationsflüsse sowie Partizipation. Die Unterfragen zur Organisationsebene sind: Leistungsvorgaben und Kontrolle, Mitbestimmung, Führung sowie Datenschutz.

Abb. 4: Ergebnisse des Screening-Tools (beispielhafter Auszug)

Die Abbildung zeigt einen Graphen mit vier Dimensionen jeweils in Himmelsrichtung. Auf den Dimensionen sind die Parameter des Screenings der Kategorie Anwendungsebene abgebildet: nach Norden die Ergebnisse zu Lernen und Verbesserung, nach Westen zu Partizipation, nach Osten zu Zusammenarbeit sowie nach Süden zu Kommunikation und Informationsflüsse. Aufgetragen sind zwei Werte: die durchschnittliche Leistung und die erforderliche Leistung durch die Gestaltungslösung. Liegt die erforderliche Leistung höher als die durchschnittliche, müssen zur erfolgreichen Umsetzung Gestaltungsmaßnahmen ergriffen werden.

Abb. 5: CeyeBERMANS Mess-System für physische und psychische Belastungen Die Abbildung zeigt eine Schaufensterpuppe mit dem Anzug mit Messsensoren. Es handelt sich um einen Overall mit Jacke und Handschuhen sowie Kappe. Auf den Kleidungsstücken sind Sensoren angebracht, die miteinander verkabelt mit einem Messrechner auf den Rücken verbunden sind. Zusätzlich trägt die abgebildete Schaufensterpuppe eine Blickbewegungsbrille, mit der das Blickverhalten gemessen werden kann.

Abb. 6: Blickbewegungs-Messsystem (links), EEG (rechts) sowie deren Kombination (Mitte) Die Abbildung zeigt einen Teilausschnitt der Schaufensterpuppe: links mit dem Blickbewegungs-Messsystem, rechts zusätzlich mit einem EEG und in der Mitte als kombiniertes System.

Abb. 7: Verfahren zur echtzeitfähigen Vorhersage und Assistenz anhand erfasster spezifischer Merkmale (nach Jennerich 2018)

Die Abbildung zeigt ein Ablaufdiagramm, wie eine Vorhersage zur Unterstützung gelingt. Auf der linken Seite ist der Prozess der Datenerfassung dargestellt. Die Datenerfassung teilt sich in die Stufen: Datenerfassung, Datenaufbereitung, Merkmalsextraktion, Datenbasis, Generierung des neuronalen Netzes und abschließend Validierung. In der Mitte wird der methodische Ansatz des neuronalen Netzes gezeigt mit einer Eingangsschicht, einer verdeckten Schicht und einer Ausgabeschicht. Auf der rechten Seite ist der Prozess der Unterstützung und Rückmeldung dargestellt mit: Echtzeiterfassung, Datenaufbereitung, Merkmalsextraktion, Import des neuronalen Netzes, Klassifizierung und abschließend Rückmeldung.

Abb. 8: Visuelle und taktile Rückmeldesysteme zu Assistenz von Personen; links visuelle Rückmeldung mit einer AR-Datenbrille; rechts haptische Rückmeldung mithilfe eines Mobiltelefons (nach Burkhardt 2019)

Die Abbildung zeigt auf der linken Seite einen Ausschnitt der Schaufensterpuppe mit einer AR-Datenbrille. Auf dem Display der Brille werden Haltungen des Menschen mit Strichmännchen abgebildet. Das linke Strichmännchen zeigt starke Verdrehungen und Verbeugungen mit Pfeilen und Kreisen an. Das mittlere Strichmännchen zeigt Belastungen des oberen Rückens durch zwei Punkte im oberen Rückenbereich. Das rechte Strichmännchen 
zeigt Belastungen des Unterarms durch zwei Punkte auf dem rechten und linken Unterarm. Auf der rechten Seite der Abbildung wird ein Mobiltelefon, das über eine Schnalle am linken Unterarm angebracht ist, gezeigt. Auf dem Display sind wieder Belastungssituationen abzulesen. Es werden zwei Belastungssituationen dargestellt. Oben: alles in Ordnung bei dem Griff von zwei gut erreichbaren Latten aus einem Regal; unten: Belastung des linken Beins durch ungünstige Griffposition beim Herausnehmen der Latten.

\section{Markus Schäfers und Viviane Schachler}

\section{Barrieren erfragen - Herausforderungen der empirischen Erfassung von Barrieren im Rahmen standardisierter Interviews}

Abb. 1: Allgemeine Bewertungen der Barrierefreiheit von Orten im Lebensumfeld (eigene Anfertigung nach Rambausek 2017, 477)

Ausschnitt aus einem schriftlichen Fragebogen. Die Frage lautet: „Wie barrierefrei ist Ihr/ Ihre Apotheke, Arzt, Bank, Bibliothek?" Als Antwort soll jeder Ort mit „gut“, „mittelmäßig“ oder „schlecht“ bewertet werden.

Abb. 2: Erfassung von Barrieren im öffentlichen Raum (eigene Anfertigung nach Schröttle $\&$ Hornberg 2014b, 72)

Ausschnitt aus einem Fragebogen. Die Frage lautet: „Gibt es für Sie Einschränkungen, Hindernisse oder Probleme im öffentlichen Raum, zum Beispiel bei der Nutzung von Bahnhöfen, Verkehrsmitteln und öffentlichen Orten wie Straßen, Plätzen oder öffentlichen Gebäuden?“ Die Frage soll mit „ja“ oder „nein“ beantwortet werden. „Weiß nicht“ oder „keine Angabe“ sind alternative Antwortmöglichkeiten.

Abb. 3: Spezielle örtliche Erfassung von Komponenten einer barrierefreien Infrastruktur (eigene Anfertigung nach Deutsches Studentenwerk 2012, 305f.)

Ausschnitt aus einem Online-Fragebogen. Die erste Frage lautet: „Haben Sie beeinträchtigungsbedingt besondere Anforderungen an Bau und Ausstattung der Hochschule, unabhängig davon ob diese Anforderungen bereits erfüllt sind?“ Die Frage soll entweder mit „keine spezifischen Anforderungen an Bau und Ausstattung“ oder mit „Ja, ich habe beeinträchtigungsspezifische Anforderungen betreffend ..." beantwortet werden. Die Ja-Antwort kann nicht allein, sondern immer nur mit einer spezifischen Anforderung gegeben werden, z.B. Zugang zu Gebäuden oder spezielle technische Ausstattung von Arbeitsplätzen usw. Bei einer Ja-Antwort folgt die zweite Frage: „Inwiefern sind diese beeinträchtigungsbedingten Anforderungen an Bau und Ausstattung an Ihrer Hochschule bereits erfüllt?" Es wird der genannte Bedarf angezeigt. Als Antwort soll dieser mit „ausreichend“, „teilweise“ oder „nicht ausreichend“ bewertet werden. Bei der Antwort „ausreichend“ oder „teilweise“ folgt die dritte Frage: „In welchen für Sie wichtigen Bereichen ist die barrierefreie bauliche Grundausstattung/ Zugänglichkeit unzureichend?“ Diese Frage kann mit „Räumlichkeiten des eigenen Fachbereichs“, „Hörsäle/Vorlesungsräume“, „Unibibliothek“ usw. beantwortet werden.

Abb. 4: Einschätzungen zur Benutzbarkeit der Infrastruktur (eigene Anfertigung nach WHO/The World Bank o.J., 1)

Ausschnitt aus einem Fragebogen. Zur Einleitung der Fragen dient: „I am going to ask you some general questions about your environment and your social relationships. I want you to answer the following questions on a scale from 1 to 5 , where 1 means very easy and 5 means very hard.“ Die erste Frage lautet: „Do places where you socialize and engage in 
community activities make it easy or hard for you to do this?" Diese Frage soll mit den genannten Abstufungen von 1 bis 5 bewertet werden. Zusätzlich kann mit „Not applicable“ geantwortet werden. Nach diesem Fragemuster folgen drei weitere Fragen: „Do the shops, banks and post office in your neighborhood make it easy or hard for you to use them?" „Does the transportation you need or want to use make it easy or hard for you to use it? „Does your dwelling, including the toilet, make it easy or hard for you to live there?"

Abb. 5: Erfassung von Barrieren bei der Mediennutzung (eigene Anfertigung nach Schröttle \& Hornberg 2014b, 77)

Ausschnitt aus einem Fragebogen für ein persönliches Interview. Die Frage lautet: „Gibt es für Sie Einschränkungen aufgrund Ihrer Behinderung oder Beeinträchtigung bei der Nutzung folgender Medien? Beim Lesen von Büchern, Zeitschriften und Zeitungen“. Die Frage soll mit „ja“, „nein“ oder „nutze ich nicht“ beantwortet werden. „Weiß nicht“ oder „keine Angabe“ sind alternative Antwortmöglichkeiten. Im Falle einer Ja-Antwort folgt die zweite Frage: „Was benötigen Sie, um Informationsmedien/Medien ohne Einschränkungen nutzen zu können?“ Für den/die Interviewer*in ist die Anweisung gegeben: „Frage offen stellen und Antworten zuordnen. “ Es werden verschiedene Rubriken aufgeführt, z.B. „Einfache/Leichte Sprache“, „Text statt Ton“ usw.

Abb. 6: Erfassung von Barrieren als Ursachen von fehlenden Aktivitäten (eigene Anfertigung nach Schäfers u.a. 2016b, 35f.)

Ausschnitt aus einem Fragebogen für ein persönliches Interview. Die erste Frage lautet: „Machen Sie Sport?“ Die Frage soll mit „ja“ oder „nein“ beantwortet werden. Im Falle einer Ja-Antwort folgt die zweite Frage: „Wie oft machen Sie Sport? Täglich, mindestens $1 \mathrm{mal}$ pro Woche, mindestens $1 \mathrm{mal}$ pro Monat oder weniger als $1 \mathrm{mal}$ pro Monat? Darauf folgt die dritte Frage: „Möchten Sie gerne mal/häufiger Sport machen?“ Die Frage soll mit „ja“ oder „nein“ beantwortet werden. Im Falle einer Ja-Antwort folgt die vierte Frage: „Was hindert Sie daran?“ Für den/die Interviewer*in ist die Anweisung gegeben: „Frage offen stellen und Antworten zuordnen“. Es werden verschiedene Rubriken aufgeführt, z.B. „zu bequem/kann mich nicht aufraffen“, „meine Beeinträchtigung/Behinderung“, „zu wenig/ keine Zeit“, „Barrieren hindern mich daran“ usw.

\section{Matthias Schmidt-Oblemann}

\section{Barrierefreie Gesundheitsversorgung - zwischen Zugänglichkeit allgemeiner Versorgung und Notwendigkeit besonderer Einrichtungen}

Tab. 1: Besondere Einrichtungen der Gesundheitsversorgung für Menschen mit (drohender) Behinderung

Die Tabelle ist eine einfache Auflistung, die folgende Einrichtungen und z.T. ihre gesetzliche Grundlage, vorwiegend im SGB V, umfasst: Sozialpädiatrische Zentren, Frühförderstellen, Medizinische Behandlungszentren für Erwachsene (MZEB), Psychiatrische Institutsambulanzen (PIA), Einrichtungen der ambulanten spezialfachärztlichen Versorgung, Hochschulambulanzen, integrierte ärztliche und therapeutische Dienste in (Förder-)Kindertagesstätten, Schulen, Berufsbildungswerken (BBW)/Berufsförderungswerken (BFW) oder Wohneinrichtungen, die Leistungen zur Erziehung, Bildung, beruflichen oder sozialen Teilhabe erbringen, soweit diese für die Erreichung der Ziele erforderlich sind (vgl. z.B. $\$ 10$ Werkstättenverordnung), Beratungsstellen für unterstützte Kommunikation so- 
wie Fachkrankenhäuser und spezialisierte Einrichtungen der medizinischen Rehabilitation, z.B. Rehabilitationseinrichtungen für psychisch Kranke, mobile Rehabilitation für mobilitätsbeeinträchtigte Menschen mit ggf. weiteren relevanten Teilhabebeeinträchtigungen.

Tab. 2: Sozialleistungsträger im Bereich der Gesundheitsversorgung

Die Tabelle listet auf: die Gesetzliche Krankenversicherung, insbesondere für ärztliche, psychotherapeutische und zahnärztliche Leistungen, Krankenhausleistungen, veranlasste Leistungen (ambulant), z.B. Arznei- und Verbandmittel, Heilmittel, Hilfsmittel zur Krankenbehandlung, häusliche Krankenpflege, Soziotherapie, Krankentransport sowie Leistungen der medizinischen Rehabilitation.

Ferner werden die soziale Pflegeversicherung, die Rentenversicherung (für Leistungen der medizinischen Rehabilitation), die Private Kranken-, ggf. Zusatz- und Pflegeversicherung, die Beihilfe sowie andere Träger wie z.B. die Unfallversicherung, Integrationsämter u.a. benannt. Die Eingliederungshilfe erbringt Leistungen der medizinischen Rehabilitation bei Fehlen eines vorrangigen Leistungsträgers, Leistungen zur sozialen Teilhabe, die der Unterstützung der behinderten Menschen bei der Gesundheitssorge dienen, als Assistenzleistungen (vgl. $\$ 78$ Abs. 1 und $\$ 113 f f$. SGB IX), heilpädagogische Leistungen, Leistungen zum Erwerb und Erhalt praktischer Kenntnisse und Fähigkeiten, Pflege, soweit sie die einfache Behandlungspflege nicht übersteigt, Hilfsmittel sowie Leistungen zur Mobilität.

Tab. 3: Leistungsspektrum der Gesetzlichen Krankenversicherung (GKV)

Die Tabelle listet Leistungen der Gesetzlichen Krankenversicherung (GKV) im Rahmen des SGB V auf, und zwar: Leistungen der ambulanten Krankenbehandlung und Prävention, z.B. hausärztliche Leistungen, fachärztliche Leistungen, psychotherapeutische Leistungen und zahnärztliche Leistungen. Als besondere Formen gelten (vgl. auch Tab. 1): Vorsorge/ Früherkennungsuntersuchungen $(\$ \$ 20 \mathrm{ff}$. SGB V) für Kinder und Jugendliche sowie Erwachsene, Disease-Management-Programme, Leistungen zur Prävention ( $\$ 20 \mathrm{ff}$. SGB V), Psychiatrische Institutsambulanzen (PIA), Sozialpädiatrische Zentren (SPZ), Medizinische Behandlungszentren für erwachsene Menschen mit Behinderung (MZEB), heimärztliche Versorgung (Pflegeheime $\$ 119 \mathrm{~b}$ SGB V), ambulante spezialfachärztliche Versorgung und Leistungen durch Hochschulambulanzen. Hinzu kommen Krankenhausleistungen einschließlich Frührehabilitation sowie veranlasste Leistungen (ambulant), z.B. Arznei- und Verbandmittel, Heilmittel, Hilfsmittel, häusliche Krankenpflege, Kurzzeitpflege (\$39c SGB V), Soziotherapie, Krankentransporte, Haushaltshilfe, nichtärztliche sozialpädiatrische Leistungen ( $\$$ 43a SGB V), MZEB ( $\$ 43$ b SGB V), sozialmedizinische Nachsorge für Kinder und Jugendliche ( $\$ 43$ Abs. 2 SGB V) und die spezialisierte ambulante Palliativversorgung (SAPV). Ferner werden Leistungen der medizinischen Rehabilitation und der medizinischen Vorsorge sowie ergänzende Leistungen zur Rehabilitation erbracht. Weitere Leistungen bestehen in der Anordnung von Hilfeleistungen anderer Personen ( $\$ 73$ Abs. 2 Nr. 6 SGB V), Bescheinigungen und Berichte für die Krankenkasse bzw. den Medizinischen Dienst der Krankenversicherung $(\mathrm{MdK})$ und andere Sozialleistungsträger.

Tab. 4: Beispiele für typische Barrieren für Menschen mit Behinderungen im Gesundheitswesen

Die Tabelle listet folgende Barrieren auf: bauliche Barrieren (Praxen von Ärzten, Therapeuten): Treppen, WC, fehlende Hilfsmittel (z.B. Lifter, Liegen/Untersuchungsstühle), komplizierte Sprache - Fehlen einfacher Sprache, Ignorierung der betroffenen Person und 
Kommunikation nur mit der Begleitperson, Unverständnis und Ungeduld bei Ängsten, Ärger bei mangelnder Kooperation, unzureichende Zeitbudgets bei der Konsultation bei allgemeinem Zeitdruck und Überlastung, fehlende Kenntnis von spezifischen Krankheitsbildern und Syndromkombinationen, mangelnde Kompetenzen bei der Symptomerkennung, v.a. bei fehlender Sprache und bei der Interpretation von Symptomen, die oft atypisch präsentiert werden, Fehlen von Gebärdendolmetschern (ggf. auch Übersetzern von Fremdsprachen), Fehlen von barrierefreien Unterlagen für Menschen mit Sehbehinderung, fehlende Unterstützung während medizinischer Maßnahmen, insbesondere im Krankenhaus, fehlende Angebote in Rehaeinrichtungen für Menschen mit intellektuellen oder mehrfachen Beeinträchtigungen oder Pflegebedürftigkeit (Ausschluss wegen mangelnder Rehafähigkeit), Mangel an mobilen Angeboten für immobile Patienten, fehlendes Fallmanagement zur Bewältigung der Barrieren bei der Inanspruchnahme von gesundheitsbezogenen Leistungen im gegliederten System der Sozialleistungen

Tab. 5: Häufige (Begleit-)Diagnosen und Symptome bei Menschen mit Behinderungen Die Tabelle listet folgende Diagnosen bzw. Symptome auf: Epilepsie, unklare Schmerzzustände, gastrooesophagealer Reflux, Obstipation und Koprostase, Inkontinenz, Decubitus, schlecht heilende Wunden, atypische Bewegungsmuster/Aufgabe vorhandener Bewegungskompetenzen, Lähmungen/Spastik, fehlende Sprache, schwere körperliche Bewegungseinschränkungen, Sinnesbeeinträchtigungen (Sehen, Hören), Kombination von intellektuellen und seelischen Behinderungen mit atypischer Symptomausprägung, paradoxe Reaktionen, herausforderndes Verhalten.

\section{Leonora Micah Jordan}

\section{Barrierefreie Beratung - Räume der Begegnung niedrigschwellig gestalten}

Abb. 1: Der Barrierefreiheitsbedarf im Kontext von Beratung (eigene Darstellung in Anlehnung an Skiba \& Züger 2017, 9)

Im Zentrum von Abbildung 1 ist ein graues Rechteck abgebildet mit dem Text: „Bedarf von Barrierefreiheit im Kontext von Beratung“. Kreisförmig herum - und jeweils mit einer Linie verbunden - werden in zwölf weiteren grau unterlegten Rechtecken unterschiedliche Formen von Teilhabebeeinträchtigungen für diesen Bedarf genannt. Oben auf zwölf Uhr beginnend und dann dem Uhrzeigersinn folgend: mobilitätsbeeinträchtigt, gehörlos, sprach-/sprechbeeinträchtigt, rollstuhlnutzend, kognitiv beeinträchtigt, (er-)blind(-et), (chronisch) erkrankt, schwerhörig, mehrfach behindert, gehbehindert, psychisch erkrankt, sehbehindert.

\section{Tanja Freifrau Schenck zu Schweinsberg (geb. Lück) und Dominik Rupprecht}

\section{Barrierefreie dialogorientierte Teilhabeplanung am Beispiel des Integrierten Teilhabeplans (ITP)}

Abb. 1: Kreislauf einer benutzerzentrierten Entwicklung (eigene Anfertigung nach DIN EN ISO 9241-210)

In Abbildung 1 ist der Kreislauf einer nutzerzentrierten Entwicklung nach DIN EN ISO 9241-210 dargestellt. In der linken oberen Ecke startet der Kreislauf mit der Planung des Gestaltungsprozesses. Ein Pfeil nach rechts führt zum ersten Knotenpunkt des Kreislaufes: Nutzungskontext verstehen und beschreiben. Es folgen im Uhrzeigersinn die Punkte Nut- 
zungsanforderungen spezifizieren, Gestaltungslösungen entwickeln und Evaluation durchführen. Vom letzten Punkt führen jeweils gestrichelte Linien zurück zu den zuvor genannten drei Punkten mit dem Hinweis, dass hier ggf. iteriert werden kann. Zudem führt vom letzten Knoten noch ein Pfeil zu einem letzten Punkt außerhalb des Kreislaufes: Lösung erfüllt Nutzeranforderungen.

Abb. 2: Screenshots aus der Szene „sich in verschiedenen Umgebungen fortbewegen “ Abbildung 2 zeigt drei Screenshot aus der Szene „sich in verschiedenen Umgebungen fortbewegen“. Die Bilder zeigen jeweils einen Mann. Auf dem ersten Bild geht er eine Treppe hinauf. Auf dem zweiten überquert er einen Hügel und auf dem dritten geht er über eine Straße.

Tab. 1: Erprobungsabschnitte des Projektes „inBEF“ in zeitlicher Reihenfolge (modifiziert und angelehnt an Lück \& Gromann 2016, 46)

In Tabelle 1 sind die sieben Erprobungsabschnitte des Projektes „inBEF“ von Mai 2013 bis März 2015 festgehalten. Pro Zeile ist einer von sieben Erprobungsabschnitten beschrieben. Es gibt drei Spalten mit den Überschriften Monat, inhaltlicher Fokus der Erprobung, Setting, die sich jeweils auf jeden Erprobungsabschnitt beziehen.

Es folgt die Erläuterung zum Inhalt der einzelnen Zeilen:

Zeile 1: Im Mai 2013 war die Erprobung der Navigations- und Grundstruktur des Prototyps inhaltlicher Fokus mittels Einzel-Settings am Computer.

Zeile 2: Im Oktober 2013 war die Erprobung statischer Erklärelemente auf Papier außerhalb des Prototyps inhaltlicher Fokus mittels eines Workshops.

Zeile 3: Im März 2014 war die Erprobung der erneuerten Navigations- und Grundstruktur inklusive implementierter statischer Erklärelemente inhaltlicher Fokus mittels Einzel-Settings am Computer.

Zeile 4: Im Mai 2014 war die Erprobung statischer und auditiver Erklärelemente implementiert im Prototyp inhaltlicher Fokus mittels Einzel-Settings am Computer.

Zeile 5: Im Juli 2014 war die Erprobung der erneuerten Navigations- und Grundstruktur mit avatarbasierten Erklärelementen zu „Fähigkeiten oder Beeinträchtigungen der Teilhabe in Selbstsorge/Wohnen, [...] Freizeit/Teilhabe an Gesellschaft" exemplarisch mit ICF-bezogenem Item „Essen“ inhaltlicher Fokus mittels EinzelSettings am Computer.

Zeile 6: Im September 2014 war die Erprobung der erneuerten Navigations- und Grundstruktur mit avatarbasierten Erklärelementen zu „Fähigkeiten oder Beeinträchtigungen der Teilhabe in Selbstsorge/Wohnen, [...] Freizeit/Teilhabe an Gesellschaft“ exemplarisch mit ICF-bezogenem Item „Essen“ in weiteren Abstufungen inhaltlicher Fokus mittels Einzel-Settings am Computer.

Zeile 7: Im März 2015 war die Erprobung avatarbasierter Erklärelemente zu „Fähigkeiten oder Beeinträchtigungen der Teilhabe in Selbstsorge/Wohnen, [...] Freizeit/ Teilhabe an Gesellschaft“ exemplarisch mit ICF-bezogenen Items „Essen“, „Einkaufen“, „sich in verschiedenen Umgebungen fortbewegen“ in weiteren Abstufungen inhaltlicher Fokus mittels Einzel-Settings am Computer. 
Christophe Kunze

Nutzerorientierte und partizipative Ansätze in Gestaltungs- und Aneignungsprozessen von teilhabefördernder Technik

Abb. 1: Beispiel für einen Wizard-of-Oz-Test mit Menschen mit Demenz zur Anwendung „Augmentierter Sitztanz“

Die Abbildung besteht aus drei Teilbildern: Das Bild links stellt vier ältere und eine jüngere Person im Stuhlkreis dar, die ihre Beine anheben. Das mittlere Bild zeigt zwei Füße einer Person in Hausschuhen auf Trittmatten. Das rechte Bild zeigt ein Smartphone mit einer Klaviatur auf dem Display und eine Hand, welche das Smartphone bedient. 



\section{k linkhardt}

978-3-7815-2418-7

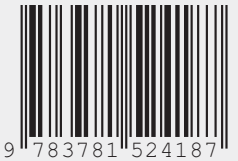

Barrierefreie Umwelten ermöglichen Menschen mit Beeinträchtigungen, am gesellschaftlichen Leben teilhaben zu können. Aber was genau bedeutet Barrierefreiheit? Was macht eine Gegebenheit in der Umwelt zu einer Barriere? Welche Aspekte entscheiden darüber, ob Umwelten förderlich oder hinderlich für die gesellschaftliche Teilhabe sind? Wie lassen sich diese Aspekte messen, beurteilen und klassifizieren? Was bedeutet das für die Planung und Beurteilung gestalteter Umwelten?

Die Beiträge des Buches nähern sich diesen Fragen aus unterschiedlichen disziplinären Perspektiven. Sie zeigen die Vielschichtigkeit und Komplexität des Themas Barrierefreiheit. Das Buch soll der Diskussion um Barrieren und Barrierefreiheit - einem noch wenig erforschten Gebiet - Substanz verleihen sowie zum interdisziplinären Dialog und zur Reflexion der Praxis inspirieren.

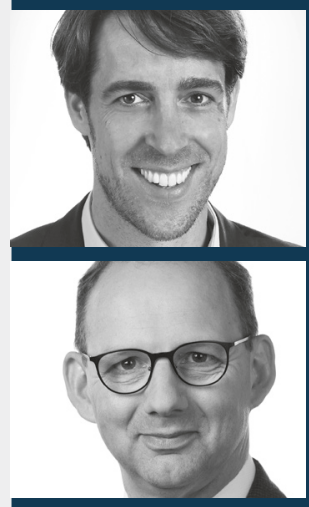

Die Herausgeber

Markus Schäfers ist Professor für Rehabilitation und Teilhabe im Sozialraumbezug am Fachbereich Sozialwesen der Hochschule Fulda. Felix Welti ist Professor für Sozialund Gesundheitsrecht, Recht der Rehabilitation und Behinderung am Fachbereich Humanwissenschaften der Universität Kassel.

Beide sind Sprecher der AG Teilhabeforschung des Forschungsverbunds Sozialrecht und Sozialpolitik der Hochschule Fulda und der Universität Kassel (FoSS). 